\title{
ROLE OF RRV ORF52 IN VIRION MATURATION
}

\author{
Melissa Sue Anderson \\ Belleville, IL
}

B.S., Northeastern Illinois University, 2007

M.S., University of Virginia, 2012

A Dissertation presented to the Graduate Faculty of the University of Virginia in Candidacy for the degree of Doctor of Philosophy

Department of Microbiology, Immunology, and Cancer Biology

University of Virginia

May, 2014 


\section{ABSTRACT}

Rhesus monkey rhadinovirus (RRV) is a close relative of the human pathogen, Kaposi's Sarcoma-Associated Herpesvirus (KSHV). Primary infection with KSHV in culture is highly inefficient and it predominantly adopts a latent phenotype, expressing only a minimal number of viral genes and producing no progeny virions. This contrasts with RRV in culture, which replicates to high viral titer and produces abundant progeny virions, making it a useful model to study gammaherpesvirus lytic replication, virion structure, and the potential roles of tegument proteins in the biology of gammaherpesviruses. Initial work in our laboratory determined that the RRV virion was composed of 33 virally encoded proteins. Of these, 17 are tegument proteins, five of which are unique to the gammaherpesvirus subfamily. ORF52 is one of these five gammaherpesvirus specific tegument proteins and is the focus of this dissertation. This protein is highly abundant within the virion (approximately 1000 copies per particle) and it is closely associated with the capsid. Our results describe a critical role for ORF52 in the cytoplasmic-based later stages of virion morphogenesis. Without ORF52, capsids fail to undergo tegumentation, which is necessary for final envelopment and production of fully infectious virions. In a later section of this dissertation, we also describe preliminary data proposing that ORF52 may play a direct or indirect role in virion transport through interaction with cellular microtubules. 


\section{ACKNOWLEDGEMENTS}

I grew up in a small, blue-collar, southern Illinois town where most people stay local and focus on getting by day-to-day, not on things like big cities and advanced degrees. I didn't even know what "graduate school" was until several years into my undergraduate education in Chicago, which, by the way, took me 9 years to complete because I worked full-time and went to school off-and-on and part-time when time and money permitted. The reason I share this is because I didn't get here alone, I didn't even know where "here" was and if I'm honest, I don't think I ever really believed I would earn a PhD, because where I'm from, things like that just don't happen to people like me. There truly are so many people I am honored to thank for helping, and supporting, me at various points in this long, wonderful, horrible, amazing, and trying journey that has been the last 10 years of my life, 7 of those at UVa.

First, I want to thank my thesis committee, Jay Brown, Amy Bouton, George Bloom, David Rekosh, and Judy White. Over the course of the past several years, I have learned to see that you really have been in my corner, fighting for my success all along. Your time, critiques, challenges, and questions have made me a better scientist and also a better person. Jay, thank you for your careful reading of my first thesis draft and your notes; they really did make my thesis a much better document. Amy, thank you for putting your foot down on yet another round of experiments using the BAC. After 2 years running blank gels, I don't think I could have taken much more. 
To my lab, past and present, including Tom Ellison, Margaret Grant, Evonne Woodson, Matt Loftus, Lynn Hassman, Lisa Nichols, Nancy Verville, and Christine O'Connor, thank you. Tom, your knowledge of the literature and molecular cloning techniques are unparalleled. I can't thank you enough for the gracious giving of your time and help in lab and also your kindness, friendship, and willingness to always help outside of lab. Also, thanks for helping me in spite of my sometimes impatient and short attitude. Margaret, thank you for reading some of my jumbled writing and giving feedback. I know it doesn't seem like much to you, but it was, and to me the biggest deal is that I was able to ask for your help when I really needed it and know that you would give it willingly. Thank you for your friendship, the margs, movies, hikes, queso, and games - this would have been a lonely couple of years if you weren't here. I'm excited to see what happens next for you. Matt, thank you for your help with my project and the IFs that are included both in the manuscript and my thesis. I know you're just doing your job, but IFs have never been fun for me and I appreciate you doing them, and doing them well. Lynn and Lisa, it's been a few years since you both left, but I appreciate your help, time, laughs, beers, and patience. Nancy started the work on ORF52 and purified ORF52 and SCIP protein so that we could have antibodies made, both have been absolutely necessary for this project. I never met Christine, but her work on the RRV virion proteins led to my work on ORF52 and I hear she was lots of fun. I was fortunate to mentor Sophia Urban, a very intelligent and highly motivated UVa pre-med undergraduate student, for 2 years. I wish I had been as consistently patient with you as you were with me. Thank 
you for your hard work and grace while I figured out how to mentor someone while in the throws of feeling like my project was going absolutely nowhere.

To Dean, the constant member, and driving force, behind the Kedes Lab - thank you. Seriously, I know I am a handful and a challenge and that I sometimes make it very hard for you to be as patient as you have been. I chose your lab to do my thesis work, because I liked the science, but also because I liked you. From the beginning, I felt like you were a no nonsense, straightforward person and I connected with that and your sense of humor played well off mine. Also, you fight for your people, and I needed to know that my PI would fight for me, and you have. I have learned so much from working in your lab, from cool research techniques, to writing a better document, to putting together a coherent presentation, and things like believing in myself and my science and the work that I have done. Again, I know it hasn't been easy, but if I had to do it all over again, l'd still pick your lab. Thank you for letting me call it 'home' for the past 6 years and for being a friend and cheerleader, as well as my boss.

I think every person has a lab that's like home away from home and for me that was the Bouton lab. Thank you to Keena Thomas, Brianne Ray, Michael Guerrero, Michael Gutknecht, Ashley Wilson, and Allison Batties. I was so fortunate to be able to come to you for supplies, help, conversations, camaraderie, and friendship. 
I appreciate the hard work and dedication of Barbee Hermann and Stacey Guillot at the University of Virginia Advanced Microscopy Core for teaching me to use the electron microscope, troubleshooting, sample preparation, and friendship. Thank you to AJ Roberts, Sandra Weirich, Eileen Trainum, and all the MIC administrative staff for their hard work and keeping things running smoothly. I also thank Eileen for her friendship, time at the Zendo, and conversations over coffee. Deepest thanks to Dr. Susan Kaufman who does her job well and who kept a welcoming space where I could find solace.

When I came to UVa, I knew I wanted to teach in some capacity and I found that opportunity at PVCC. I am so beyond grateful for that experience because not only do I really love teaching and forming relationships with students, but teaching at PVCC got me through some of the really hard, trying, and kind of heart-wrenching years in grad school. Teaching at PVCC helped me feel not terrible at something at a time when I felt nothing but terrible. I appreciate the time and mentorship of Patricia Franklin, Marlena Yost, and Joanna Vondrasek. I would also like to thank my students - each and every one of them that I came in contact with - over the 2 years that I taught at PVCC - they all taught me something about myself, science, and people in general and I'm grateful. I would like to give a special thanks to a couple of former students that I am still in contact with and am fortunate enough to consider friends: Kristian Harlan, Stacey Mitchell, and Nolan Scheible. I'm so grateful that you all walked in to my classrooms and I thank you for all you have taught me. I wish all of you the 
absolute best in whatever you do next. To Kristian, your hard work and compassion don't go unnoticed. Thank you for the fun times, for supporting me, and for letting me get to know you. You're going to be an incredible nurse - lucky us.

I would also like to thank the people who helped me along the way to do the things I needed to do that got me here. My heartfelt thanks to Patricia Bialek, aka "Chicago mom", who was, first my supervisor during my years in property management in Chicago, and more importantly my friend and mentor. Thank you for welcoming me into your family, for your support, for the car I loved, and for taking care of me as well as you did. I am indebted and so grateful to my undergraduate mentor, Dr. Terrance Puryear at Northeastern Illinois University. Dr. P saw something in me when I knew pretty much nothing about science or research. He agreed to mentor me and oversee a research project at a school where undergrads didn't do any research. He ordered supplies, taught me tissue culture and basic research techniques, reviewed my undergraduate honors thesis, and wrote about 15 letters of recommendation to graduate schools. Dr. Kimberly Sanborn was in charge of the McNair Scholars program at Northeastern Illinois University - a program that helps minority students (in my case, first generation and low-income) who want to go to graduate school - prepare. This program gave me funding to buy the supplies necessary to do my undergraduate research project. Through this program, I also received free GRE preparation, which I wouldn't have been able to afford otherwise. Dr. Jerome Langer at 
Rutgers University was my small group leader during an internship I did in 2006 for RISE at Rutgers, a summer program for minority students to gain research experience. During this 8-week program I lived with 3 (much younger) undergrads, worked on a project full-time in the lab of Dr. Paul Copeland, met with a small group to learn how to talk about science, had more GRE prep, and gave my first ever research presentation. I also got to live in $\mathrm{NJ}$, hang out with different people from all over, and have something that resembled the college experience I never had before as a part-time commuter student. This was an incredible experience and it truly fostered my excitement about science and my desire to pursue research. Dr. Langer has continued to be a source of support, guidance, and friendship and I am grateful. Also, thank you to Kelvin Caban, the then-graduate student, in Dr. Paul Copeland's lab who directly worked with me on a daily basis. I didn't realize how difficult it was to be a graduate student in the middle of a project, trying to work out issues, and also mentor a student who knew very little. I think that's one of Kelvin's superpowers, his ability to help people, me in particular, while never mading me feel like a pest or an inconvenience. I can't say that l've always lived up to his example in my own experiences, but I try. I'm still in touch with Drs. Puryear, Sanborn, Copeland, and Langer and I'm forever grateful for their time, patience, help, and support. Finally, I would like to thank my undergraduate World Of Fiction instructor in 2003 whose name I cannot remember. Regardless, this woman was a hardcore instructor, who for some unknown reason saw something in me, pulled me aside after class one day and recommended that I apply for the university honors 
program. As a part-time student, I felt disconnected from the school and like no one knew me, so this woman seeing me and directing me to something bigger affected my life. Without her, I may have been bumbling around letting myself be a 'C' student, because it was easy. Instead, l've spent the last 11 years pushing myself to live up to something she, and the handful of other key people, saw in me. I hope they're proud.

To my friends, my heart overflows with gratitude and love for you and your role in my life. My very best friend, Joy Tanksley, to say that I couldn't have done this without you, would be a massive understatement. I honestly can't thank you enough for your friendship, love, support, encouragement, thoughtful notes and packages, hand-holding, time, and genuine care. You believed in me when I most certainly did not. You knew I could do this all those times when I almost left and you would have supported me if that were my decision. You always had time and space in your heart (and home) for me no matter what was happening in your own life and I am truly grateful. Thank you for helping me believe that people really can love me at my worst, because you have seen it (repeatedly) and are still here. Thank you for always seeing me. I love you. To ALF, thank you for your support and love in a time when I desperately needed it, but couldn't accept it. I won't ever forget you. Brianne Ray, thank you for being the best running partner I could have hoped for. I loved having that experience with you and those talks were life saving. Thanks also for introducing me to Bikram yoga, it was good for me when I needed something other than running. Michael 
Guerrero, I'm so grateful I got to share TC with you for those few years. Your sense of humor, honesty, and openness won't ever be forgotten. If I had to run around the track with anyone, I'm glad it was with you. Mike Davis, thank you for your friendship, the chats and lunches, for taking me to my first (and only) UVa football game, sharing Lady Gaga live, and the cookbook that I still use. Erica Berzin, thank you for all of our talks, studying together, and letting me get to know you. Your strength and bravery are inspiring. Jessica Edelson, I'm so grateful that you and I became friends, went to MBSR together, and got to have so many talks that were absolutely crucial for my sanity. Your openness and authenticity are inspiring. Megan Lenherr, thank you for letting me be in your life and for being in mine. You are so strong and I'm so proud of you. I'm looking forward to seeing all the things that come for you. My Chicago friends: Marni Feig, Mariska Krause, Carmen Barnes, and Martii Kuznicki - thank you for still being in my life all these years later. I'm lucky to call you my friends.

To Evonne Woodson, my twin, the person I sat 6 inches away from for 5 years and saw almost everyday...there is pretty much nothing I can say to accurately express my gratitude to you. Thank you for being such an excellent role model, for being my friend, for helping me in lab and outside, for sharing your gum, and for teaching me not to judge people so quickly. I think I would have left a few times if it weren't for you sitting 6 inches away and always being willing to listen. Thank you for believing in me, for sharing your life with me, for letting me be part of your work, and for still being my friend. So many of my favorite grad school 
memories involve you and I haven't laughed nearly as hard since you've been gone. I am forever grateful for you.

To my amazing, intelligent, hilarious, one-of-a-kind, OTP, Mary Maxfield - - thank you. I'm so grateful that l've been able to share these last 2 years with you. I know that it hasn't always been easy, but I think that we've done a good job of making the most out of it. I am so grateful for your kindness, love, compassion, support, and willingness to wake up at 3am to draw cartoons. I am always inspired by your courage and realness and I love that you make me want to be a better me. Thank you for always seeing me and loving me no matter what. Thank you for your help editing and reviewing my manuscript and thesis, for dealing with computer nonsense so I didn't have to, and for always being there and ready to give the best hugs. I'm excited to move forward and see what comes next and I'm so glad that we get to do it together. I love you.

Thank you to my family, my mom and dad, Bob and Julie Anderson, who love me unconditionally and support me always. I know they don't always understand what I'm doing or why I'm doing it, but they're always cheering for me anyway. To my brother, Mike, and my sister, Ellie, I hate that I missed out on so much of your lives by being so far away, but I hope that maybe it will help you to remember that there is a great big world out there and that you really can do anything. I'm also grateful that my Papa, Pat Lee, is able to see this day happen and while my Nana, Jean Lee, is gone, she is never forgotten. I love you all. 


\section{TABLE OF CONTENTS}

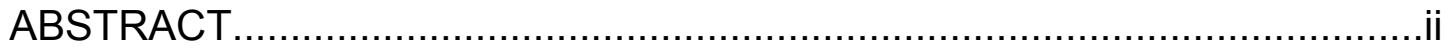

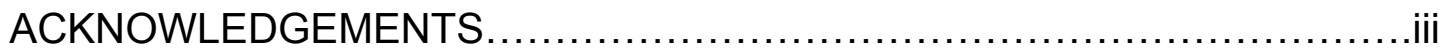

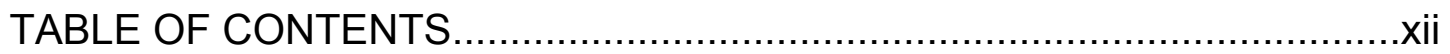

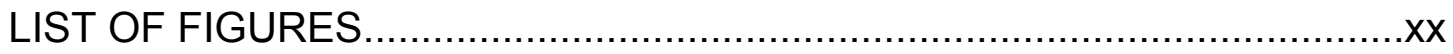

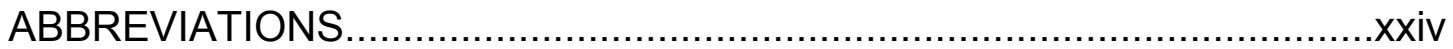

CHAPTER 1. GENERAL INTRODUCTION TO THE HERPESVIRIDAE, THE VIROLOGY OF TWO CLOSELY RELATED GAMMA-2

RHADINOVIRUSES: KAPOSI'S SARCOMA-ASSOCIATED

HERPESVIRUS (KSHV) AND RHESUS MONKEY

RHADINOVIRUS (RRV), AND AN OVERVIEW OF

GAMMAHERPESVIRUS TEGUMENT PROTEINS AND THEIR

FUNCTIONS ...................................................

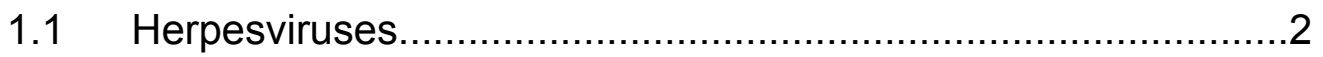

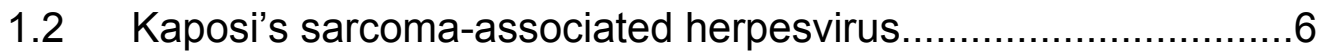

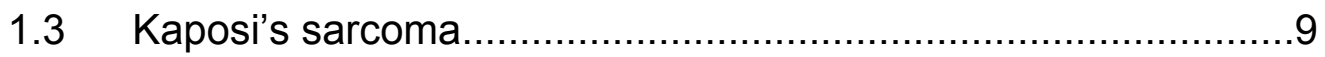

1.4 Rhesus monkey rhadinovirus (RRV) .................................11

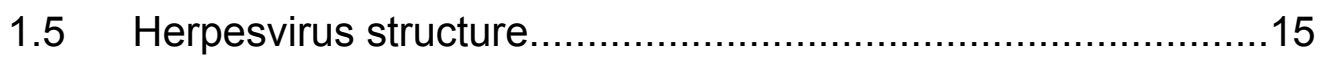

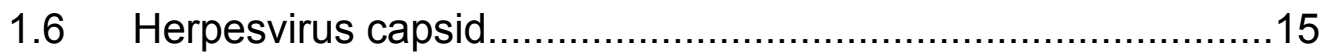

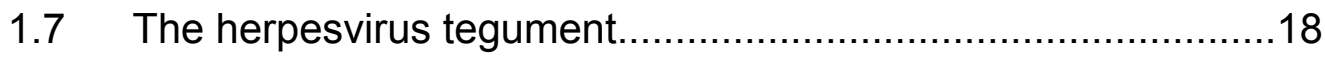

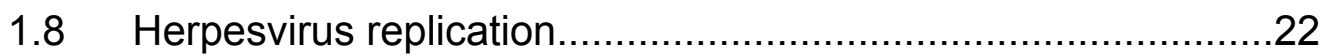

1.9 Gammaherpesvirus specific tegument proteins.....................39 


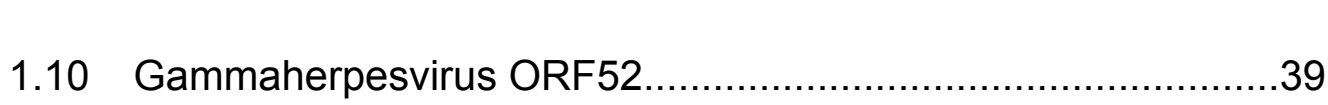

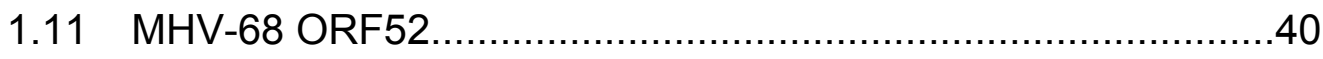

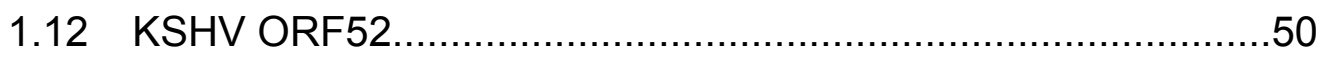

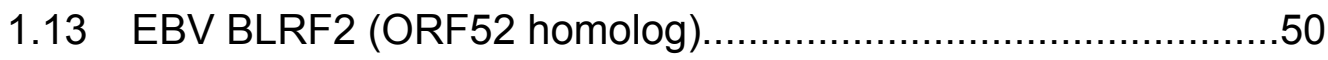

1.14 GAMMAHERPESVIRUS ORF45........................................

1.15 RRV ORF45

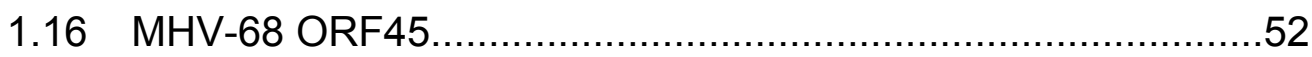

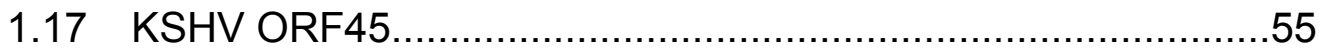

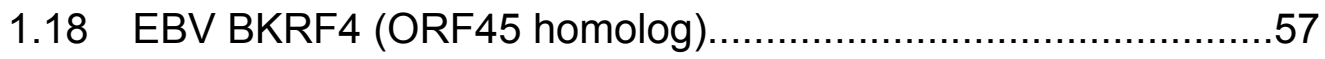

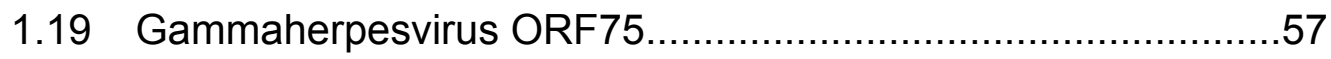

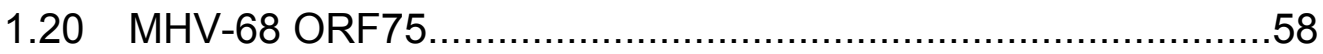

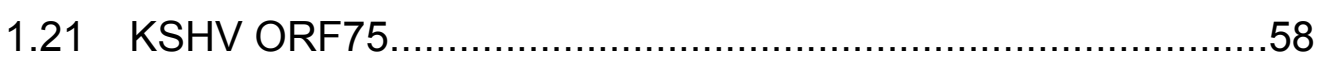

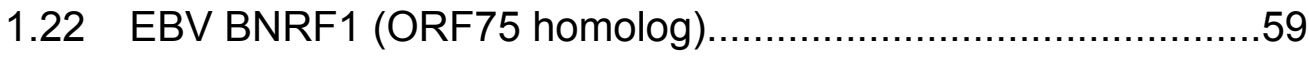

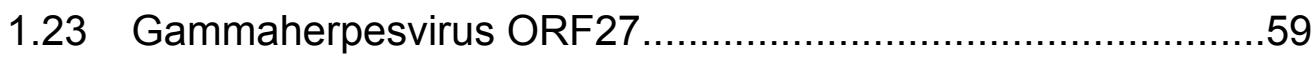

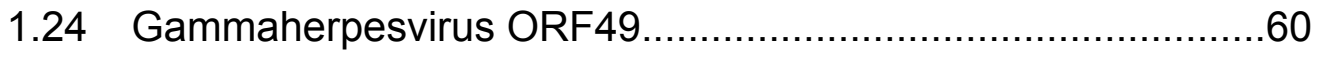

CHAPTER 2. MATURATION AND VESICLE-MEDIATED EGRESS OF PRIMATE GAMMAHERPESVIRUS RRV REQUIRE INNER

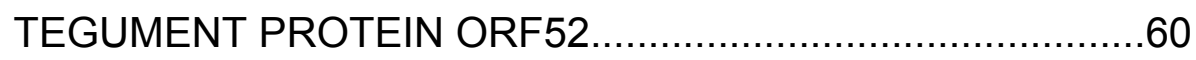

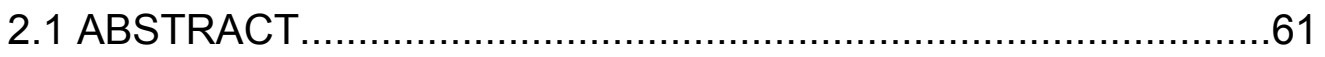

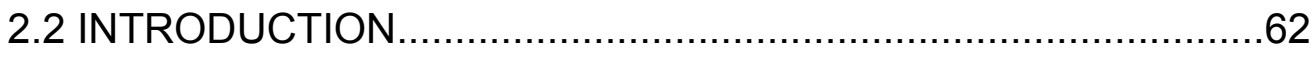

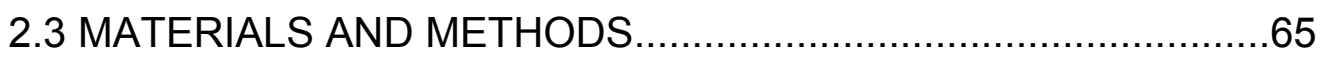

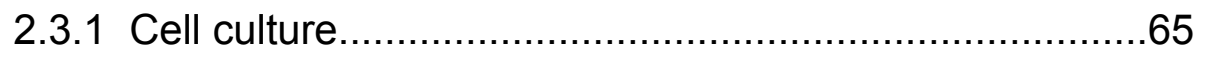


2.3.2 RRV stocks.

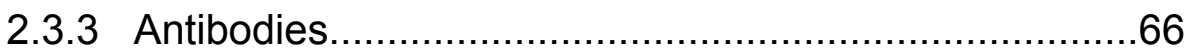

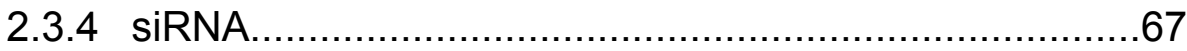

2.3.5 siRNA transfection followed by RRV infection................67

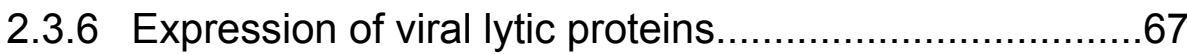

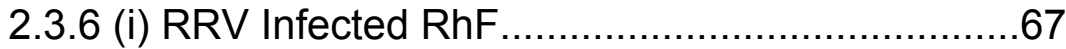

2.3.6 (ii) Viral Supernatants........................................68

2.3.7 Protein electrophoresis and immunoblotting..................68

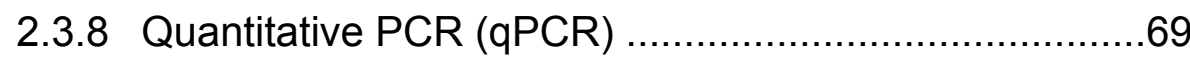

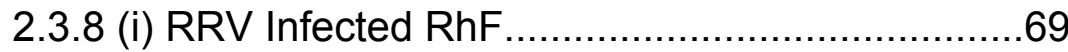

2.3.8 (ii) Viral supernatants......................................

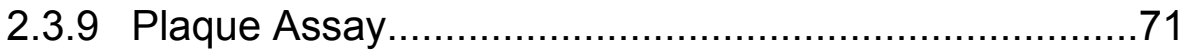

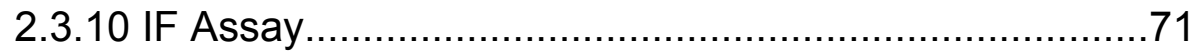

2.3.11 Transmission Electron Microscopy ................................73

2.3.10 (i) RRV infected RhF.......................................

2.3.10 (ii) Supernatants............................................73

2.3.12 Complementation of RRV ORF52 with exogenous

siORF52 resistant plasmid.........................................

2.3.13 Confirmation of Res52 expression and resistance to

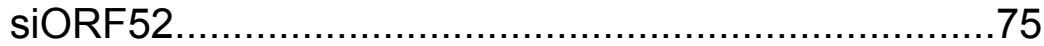

2.3.14 Complementation assay ...........................................

2.3.15 IF Assay for Complementation...................................76

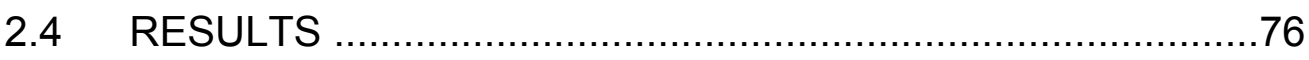


2.4.1 Efficient knockdown of ORF52 has little effect on other

viral structural proteins............................................

2.4.2 Loss of ORF52 markedly decreases viral titer...............77

2.4.3 Release of an increased proportion of empty particles

2.4.4 ORF52 knockdown led to the release of subviral particles lacking tegument.

2.4.5 Loss of ORF52 prevents ORF45 incorporation into the particle.

2.4.6 Secondary envelopment depends on ORF52. .91

2.4.7 ORF52 is necessary for tegumentation and envelopment.

2.4.8 Efficient release and cell-surface association of viral particles are ORF52 dependent.

2.4.9 Expression of siRNA-resistant ORF52 in trans partially rescued maturation and release of infectious virions in ORF52 knockdown cells. 102

2.4.10 ORF52 in trans partially rescued virion maturation.....107

2.4.11 Res52 partially restored nuclear egress of ORF45 in siORF52 treated cells

2.5 DISCUSSION.

2.5.1 Increased proportion of immature particles in the absence of ORF52 
2.5.2 ORF52 is essential for virion maturation following egress from the nucleus

2.5.3 ORF52 acts as a functional lynch pin for tegument formation

2.5.4 Nuclear egress of ORF45 is ORF52 dependent

2.5.5 Inability of particles to invaginate into cytoplasmic vesicles.

2.5.6 ORF52 dependent tegumentation and envelopment are necessary for cell-surface association of viral particles.

2.5.7 ORF52 in trans partially rescues block in particle maturation

\section{CHAPTER 3. RRV ORF52 COLOCALIZES WITH MICROTUBULES AND} INDUCES A BUNDLING PHENOTYPE.

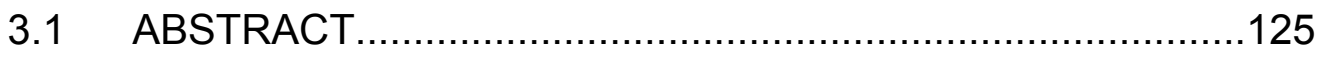

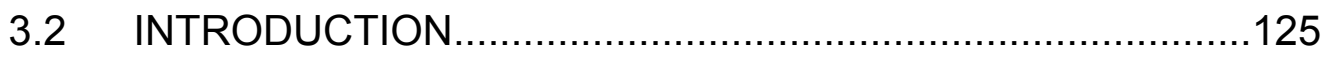

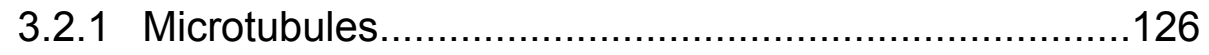

3.2.2 Microtubule associated proteins................................129

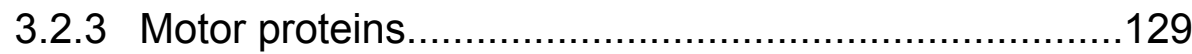

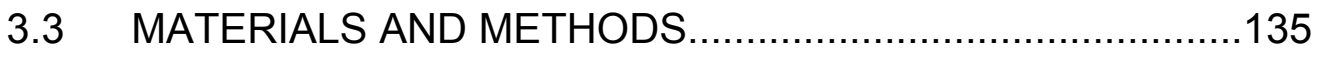

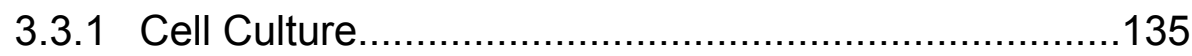

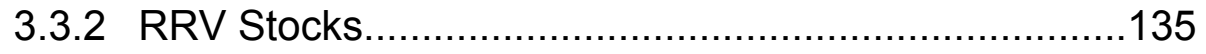


3.3.3 ORF52 plasmid and generation of arginine to alanine

mutant plasmid.

3.3.4 Immunofluorescence assay (IFA)

3.3.5 Transfection of ORF52

3.3.6 Microtubule spin-down assay.

3.3.6 (i) Polymerization of MTs for purified binding assay

3.3.6 (ii) MT binding assay using purified MTs and purified ORF52.

3.3.6 (iii) MT pull-down assay in transfected cells

3.4 RESULTS AND DISCUSSION

3.4.1 RRV ORF52 colocalizes with microtubules and induces a bundle-like microtubule phenotype. 141

3.4.2 RRV infection also leads to an ORF52 staining pattern that resembles cytoskeletal MTs. 144

3.4.3 Purified ORF52 weakly interacts with purified MTs......144

3.4.4 ORF52 MT binding in transfected cell extracts is dependent on MT polymerization. 149

3.4.5 Conserved ORF52 residue, arginine 95 (R95) (R103 in RRV), may play a role in MT thickening phenotype....149

3.5 Summary. 154 


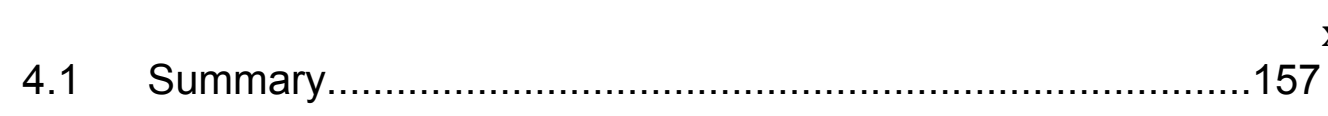

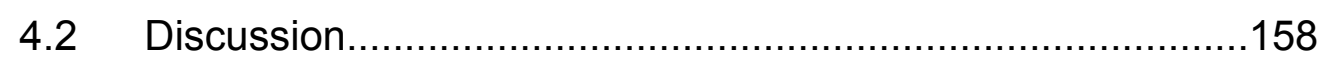

4.2.1 The role of ORF52 in the protein-protein interactions leading to the structure of the virion...........................158

4.2.2 When is ORF52 added to the particle?.......................163

4.2.3 The role of ORF52 in secondary envelopment............164

4.2.4 Domains of ORF52 responsible for its functions in both the RRV life cycle and MT interaction........................166

4.2.5 Release of subviral viral particles in ORF52 kd...........167

4.2.6 Role of ORF52 in interaction with MTs and movement

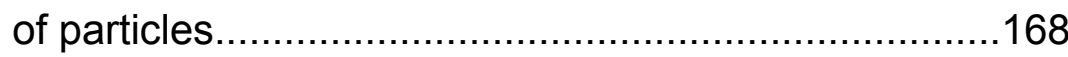

4.2.7 Phosphorylation of ORF52 and interaction with SRPK2.

4.2.8 Creation of a RRV BAC - wt, 52- stop, 52-revertant .....171

4.2.9 Does RRV ORF52 play a role following virus entry?.....172

4.2.10 The tegument and its potential role as a therapeutic target. 173

4.2.11 Model of virion maturation with, and without ORF52...174

APPENDIX 1. RRV bacterial artificial chromosome (BAC)...............178 APPENDIX 2. ShORF52 and shScrambled expressing RhF cell lines. .194 
APPENDIX 3. Tet-inducible ORF52 and siORF52 resistant RhF cell

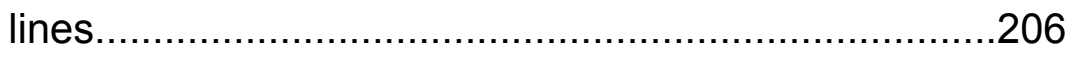

APPENDIX 4. ORF45 and its role in the sustained activation of ERK

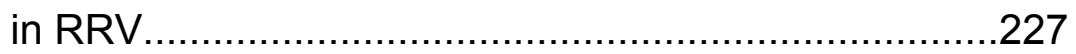

APPENDIX 5. Effect of RRV ORF45 knockdown on virus

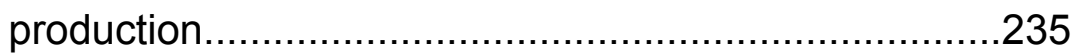

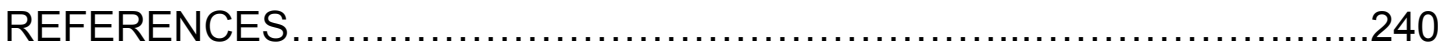


LIST OF FIGURES

Figure 1-1 Classification of herpesviruses in the family Herpesviridae........3

Table 1-1 Potential KSHV genes involved in tumorgenesis......................7

Figure 1-2 Alignment of ORFs of KSHV and RRV26-95 .......................13

Figure 1-3 Herpesvirus structure ..................................................16

Figure 1-4 Cyro-electron tomogram reconstruction of MHV-68 virion.......20

Figure 1-5 Lytic replication cycle of herpesviruses........................24

Figure 1-6 Diagram of the four models for exit of herpesvirus capsids

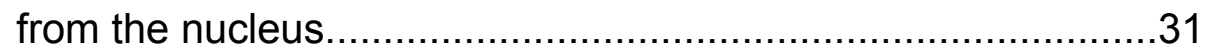

Figure 1-7 Model for virus envelopment via endocytosis....................37

Figure 1-8 Alignment of ORF52 in RRV, KSHV, MHV-68, and EBV........41

Figure 1-9 Structure of MHV-68 ORF52 dimer...........................44

Figure 1-10 Asymmetric tetramer of MHV-68 ORF52 ............................46

Figure 1-11 Alignment of ORF45 in RRV, KSHV, MHV-68, and EBV........53

Figure 2-1 Efficient knockdown of ORF52 significantly decreased RRV titer with only minimal effects on intracellular levels of other

structural viral proteins...............................................

Figure 2-2 Intracellular viral DNA production and packaging within

particles appeared independent of ORF52

Figure 2-3 In the absence of ORF52, RRV infection led to release of immature particles lacking tegument............................. 85

Figure 2-4 ORF52 knockdown restricted ORF45 to nucleus.....................89

Figure 2-5 TEM images of the concentrated media from RRV-infected 
RhF demonstrated release of untegumented and unenveloped capsid-like particles, many of which lacked DNA..................92

Figure 2-6 TEM of RRV infected cells following ORF52 knockdown showed block in tegumentation and secondary envelopment, leading to peri-vesicular accumulation of capsids as well as a lack of cell surface-associated virions.......................................

Table 2-1 Distribution of intracellular and released viral and subviral particles following RRV infection with and without ORF52 knockdown. .99

Figure 2-7 Complementation of ORF52 knockdown with exogenous siRNA-resistant ORF52 partially rescued wild-type phenotype

Figure 2-8 Exogenous ORF52 rescues formation and release of tegumented and enveloped virions

Figure 2-9 Complementation with siRNA-resistant ORF52 partially restored cytoplasmic subcellular localization of ORF45.

Figure 3-1 The structure of a microtubule and its subunit...............127

Figure 3-2 Structure of common motor proteins...........................131

Figure 3-3 Organization of microtubules in an unpoloraized fibroblast or epithelial cell. 133

Figure 3-4 Ectopic expression of ORF52 in transfected cells colocalizes with and induces a bundle-like MT phenotype. 142 
Figure 3-5 Some ORF52 has a MT-like staining pattern following RRV

infection

Figure 3-6 Purified ORF52 weakly associates with purified

microtubules

Figure 3-7 ORF52 MT binding is dependent on MT polymerization.........150

Figure 3-8 Arginine to alanine change in C-terminus of RRV ORF52

reduces MT bundling.

Figure 4-1 Interactome map of KSHV virion proteins.

Figure 4-2 Model highlighting impact of ORF52 knockdown and its effect

on virion maturation

Figure A1-1 BAC DNA purified from E. coli. 181

Figure A1-2 Lack of CPE evidence in RhF transfected with $\triangle 52 \mathrm{BAC}$ .183

Figure A1-3 Sequencing alignment of $\triangle 52 \mathrm{BAC}$ compared to wt RRV H25-95 ORF52

Figure A1-4 Examination of viral proteins expressed in WT BAC and $\triangle 52$ BAC transfected RhF cells. 190

Figure A1-5 Time course of ORF52 protein expression in HEK293 cells transfected with WT BAC or $\triangle 52 \mathrm{BAC}$ 192

Figure A2-1 Overview of lentiviral creation and shRNA processing.........196

Figure A2-2 Map of lentiviral expression vector pLKO hygro...................198

Figure A2-3 Diagram of shRNA construct including shORF52 and shScrambled oligosaccharide design sequences. .201

Figure A2-4 sh52 knocks down expression of transfected pk-mycORF52; 
however, knockdown is not stable.

Figure A3-1 Establishment of a stably transfected pFRT/LacZeo RhF

host cell line.

Figure A3-2 $\beta$-galactosidase activity in RhF cells with a FRT/lacZeo

integration

Figure A3-3 Tet-inducible RhF clones express ORF52 upon induction by doxycycline

Figure A3-4 Tet-inducible siResistant ORF52c RhF cells express ORF52 following induction with Dox

Figure A3-5 Tet-inducible siResistant ORF52c RhF cells express ORF52 upon addition of Dox and it is resistant to knockdown by siORF52c.

Figure A3-6 Expression of viral proteins in RhF cells and in released particle following ORF52 knockdown and rescue

Figure A3-7 Titers of siCNL, ORF52 knockdown, or ORF52 rescue determined by plaque assay 48-, 72-, or $96 \mathrm{hpi}$ .224

Figure A4-1 The ORF45-containing complex predominantly protects $\mathrm{pERK} 2$ over pERK1 230

Figure A4-2 Delayed decay of pERK2 during RRV infection is independent of RSK expression 233

Figure A5-1 RRV ORF45 plays a role in multiple stages of the RRV

life cycle 237 


\section{LIST OF ABBREVATIONS}

${ }^{\circ} \mathrm{C}$

$\mu \mathrm{g}$

$\mu \mathrm{l}$

$\mu \mathrm{m}$

$\mu \mathrm{M}$

$x \mathrm{~g}$

AAA

$\mathrm{Ab}$

AIDS

ATP

BAC

BCBL

BST2

C-terminal

$\mathrm{cm}^{2}$

CMV

CPE

cryoEM

DAPI

$\mathrm{DHC}$

DIC

DMSO

DNA

DNase

ds

DTT

EBV

EE

ELISA degrees Celsius

microgram

microliter

micron

micromolar

gravitation force

ATPases associated with diverse cellular activities

antibody

acquired immunodeficiency syndrome

adenosine triphosphate

bacterial artificial chromosome

body cavity based lymphoma

bone marrow stromal antigen 2

carboxyl terminal

centimeters squared

cytomegalovirus

cytopathic effect

cryo-electron microscopy

4',6-diamidino-2-phenylindole

dynein heavy chain

dynein light chain

dimethyl sulfoxide

deoxyribonucleic acid

deoxyribonuclease

double stranded

dithiothreitol

Epstein Barr virus

early endosome

enzyme linked immunosorbant assay 


\begin{tabular}{ll} 
FGARAT & phosphoribosylformylglycineamide amidotransferase \\
FITC & fluorescein isothiocyanate \\
FLP & flippase \\
FRET & fluorescence energy transfer \\
FRT & flippase recombination target site \\
g(X) & glocyprotein, where $X$ is the name/number of the protein \\
GA & golgi apparatus \\
GAPDH & glyceraldehyde 3-phosphate dehydrogenase \\
GFP & green fluorescent protein \\
GTP & guanosine-5'-triphosphate \\
HCMV & human cytomegalovirus \\
HEK293 & human embryonic kidney 293 cell line \\
HeLa & Henritta Lack cervical cancer cell line \\
HHV-6 & human herpesvirus-6 \\
HHV-7 & human herpesvirus-7 \\
HIV & human immunodeficiency virus \\
h p.i. & hours post infection \\
HRP & horseradish peroxidase \\
HSV-1 & herpes Simplex Virus-1 \\
HSV-2 & herpes Simplex Virus-2 \\
HVS & herpesvirus saimiri \\
I & induced \\
IF & intermediate filaments \\
IF & immunofluorescence \\
IFA & immunofluorescence assay \\
IgG & immunoglobulin G \\
IKKE & inhibitor of nuclear factor kappa-B kinase epsilon \\
ImmunoEM & immunogold labeling coupled with electron microscopy \\
INM & inner nuclear membrane \\
IP & immunoprecipitation \\
\hline
\end{tabular}




$\begin{array}{ll}\text { IRF-7 } & \text { interferon-regulatory factor } 7 \\ \text { KAP } & \text { kinesin associated protein } \\ \text { kb } & \text { kilobase } \\ \text { kd } & \text { knockdown } \\ \text { kDa } & \text { kilodalton } \\ \text { KHC } & \text { kinesin heavy chain } \\ \text { KIF3A } & \text { kinesin-like protein 3A } \\ \text { KLC } & \text { kinesin light chain } \\ \text { KS } & \text { Kaposi's sarcoma } \\ \text { KSHV } & \text { Kaposi's sarcoma-associated herpesvirus } \\ \text { LANA } & \text { latency associated nuclear antigen } \\ \text { LPD } & \text { lymphoprolferative disorder } \\ \text { LTP } & \text { large tegument protein } \\ \text { LUR } & \text { long unique region } \\ \text { mA } & \text { milliamp } \\ \text { MAP } & \text { microtubule associated protein } \\ \text { MCD } & \text { multicentric Castleman's disease } \\ \text { MCP } & \text { major capsid protein } \\ \text { MEK } & \text { MAPK/Erk kinase } \\ \text { MHV-68 } & \text { murine gammaherpesvirus-68 } \\ \text { MKP } & \text { MAP kinase phosphatases } \\ \text { mL } & \text { milliliters } \\ \text { mM } & \text { milimolar } \\ \text { mRNA } & \text { messenger RNA } \\ \text { MS } & \text { mass spectrometry } \\ \text { MT } & \text { microtubules } \\ \text { N-terminal } & \text { amino terminal } \\ \text { NCBI } & \text { National Center for Biotechnology Information } \\ \text { NERPC } & \text { New England Regional Primate Research } \\ \text { NGS } & \text { normal goat serum } \\ & \end{array}$




$\begin{array}{ll}\text { NHL } & \text { non-Hodgkin's lymphoma } \\ \text { nm } & \text { nanometer } \\ \text { ONM } & \text { outer nuclear membrane } \\ \text { OPN } & \text { ortho-Nitrophenyl } \\ \text { ONPG } & \text { ortho-Nitrophenyl- } \beta \text {-galactoside } \\ \text { ORF(X) } & \text { open reading frame, where } X \text { represents the gene number } \\ \text { ORPRC } & \text { Oregon Regional Primate Research Center } \\ \text { PACK } & \text { packaging protein } \\ \text { PBS } & \text { phosphate buffered saline } \\ \text { PEL } & \text { primary effusion lymphoma } \\ \text { PEV } & \text { primary enveloped virion } \\ \text { PFA } & \text { paraformaldehyde } \\ \text { PFU } & \text { plaque forming units } \\ \text { pl } & \text { isoelectric point } \\ \text { PM } & \text { plasma membrane } \\ \text { PORT } & \text { portial } \\ \text { PRO } & \text { protease } \\ \text { PrV } & \text { pseudorabies virus } \\ \text { PVDF } & \text { polyvinylidene fluoride } \\ \text { qPCR } & \text { quantitative polymerase chain reaction } \\ \text { RER } & \text { rough endoplasmic reticulum } \\ \text { RhF } & \text { rhesus monkey fibroblasts } \\ \text { RISC } & \text { RNA-induced silencing complex } \\ \text { RNA } & \text { ribonucleic acid } \\ \text { RPM } & \text { revolutions per minute } \\ \text { RRV } & \text { rhesus monkeys rhadinovirus } \\ \text { RS } & \text { serine-arginine domain } \\ \text { RSK } & \text { ribosomal S6 kinase } \\ \text { RT } & \text { room temperature } \\ \text { SCIP } & \text { small capsomer interacting protein } \\ \end{array}$




$\begin{array}{ll}\text { SD } & \text { standard deviation } \\ \text { SDS-PAGE } & \text { sodium dodecyl sulfate polyacrylamide gel electrophoresis } \\ \text { SEM } & \text { standard error of the mean } \\ \text { ShRNA } & \text { short hairpin RNA } \\ \text { SIAH } & \text { seven in absentia homolog } \\ \text { siRNA } & \text { small interfering RNA } \\ \text { SIV } & \text { simian immunodeficiency virus } \\ \text { SRPK2 } & \text { serine-arginine protein kinase } 2 \\ \text { T } & \text { triangulation number } \\ \text { TBK1 } & \text { TANK binding kinase 1 } \\ \text { TBS } & \text { tris buffered saline } \\ \text { TBST } & \text { TBS + tween } \\ \text { TEM } & \text { transmission electron microscope } \\ \text { TET } & \text { tetracycline } \\ \text { TET-R } & \text { tetracycline repressor } \\ \text { TGN } & \text { trans-golgi network } \\ \text { TR } & \text { terminal repeats } \\ \text { TRI-1 } & \text { triplex-1 } \\ \text { TRI-2 } & \text { triplex-2 } \\ \text { UI } & \text { uninduced } \\ \text { UL(X) } & \text { unique long, where } X \text { represents the gene name } \\ \text { VFLIP } & \text { viral FLICE-inhibitory protein } \\ \text { VGPCR } & \text { viral G-protein coupled receptor } \\ \text { VIL-6 } & \text { viral interlukin-6 } \\ \text { VP(X) } & \text { viral protein, where } X \text { represents the protein name } \\ \text { VZV } & \text { varicella zoster virus } \\ \text { wt } & \text { wild type } \\ & \end{array}$


CHAPTER 1. GENERAL INTRODUCTION TO THE HERPESVIRIDAE, THE VIROLOGY OF TWO CLOSELY RELATED GAMMA-2 RHADINOVIRUSES: KAPOSI'S SARCOMA-ASSOCIATED HERPESVIRUS (KSHV) AND RHESUS MONKEY RHADINOVIRUS (RRV), AND AN OVERVIEW OF GAMMAHERPESVIRUS TEGUMENT PROTEINS AND THEIR FUNCTIONS 


\section{Herpesviruses}

Viruses that make up the Herpesviridae belong to a large family of double stranded DNA viruses that are approximately $200 \mathrm{~nm}$ in diameter. These viruses have large genomes $150-200 \mathrm{~kb}$, with $70-200$ predicted open reading frames (ORFs) (reviewed in $(133,265,299))$. All members within the family share the capacity for both latent and lytic stages of infection. In the latent stage of the herpesvirus life cycle, only a small number of viral genes are transcribed and no virions are made. This is in contrast to the lytic state, when the virus expresses the full array of viral genes and produces new progeny virions (reviewed in (29, 117, 211)). The 130 known members within the family Herpesviridae all establish life-long infections within the host, have complex virion structures, and are known to infect a variety of human and non-human animals causing a variety of diseases. The Herpesviridae family comprises alphaherpesvirinae ( $\alpha$ ), betaherpesvirinae $(\beta)$, and gammaherpesvirinae $(Y)$, separated based on their genomic organization, nucleotide and amino acid sequence homology, as well as biological properties (Figure 1-1) $(63,69)$.

While these viruses share common structural features and a number of genes share high levels of homology, each encodes a subset of viral genes that are specific to that subfamily. These variances between subfamilies may play an important role in the cellular tropism and subsequently the type of pathogenesis caused by each virus. To date, investigators have identified eight herpesviruses that infect humans $(20,270)$. Members of the alphaherpesvirus subfamily include herpes simplex virus-1 (HSV-1) and herpes simplex virus-2 (HSV-2), the 
Figure 1-1. Classification of herpesviruses in the family Herpesviridae. Phylogenetic tree depicting the three subfamilies of herpesviruses: alpha, beta, and gamma. (Not depicted are the allos and maculos herpesviruseses because they are members of the Alloherpesviridae and Malacoherpesviridae families respectively and not Herpesviridae.) Image from (63) and used with permission. 


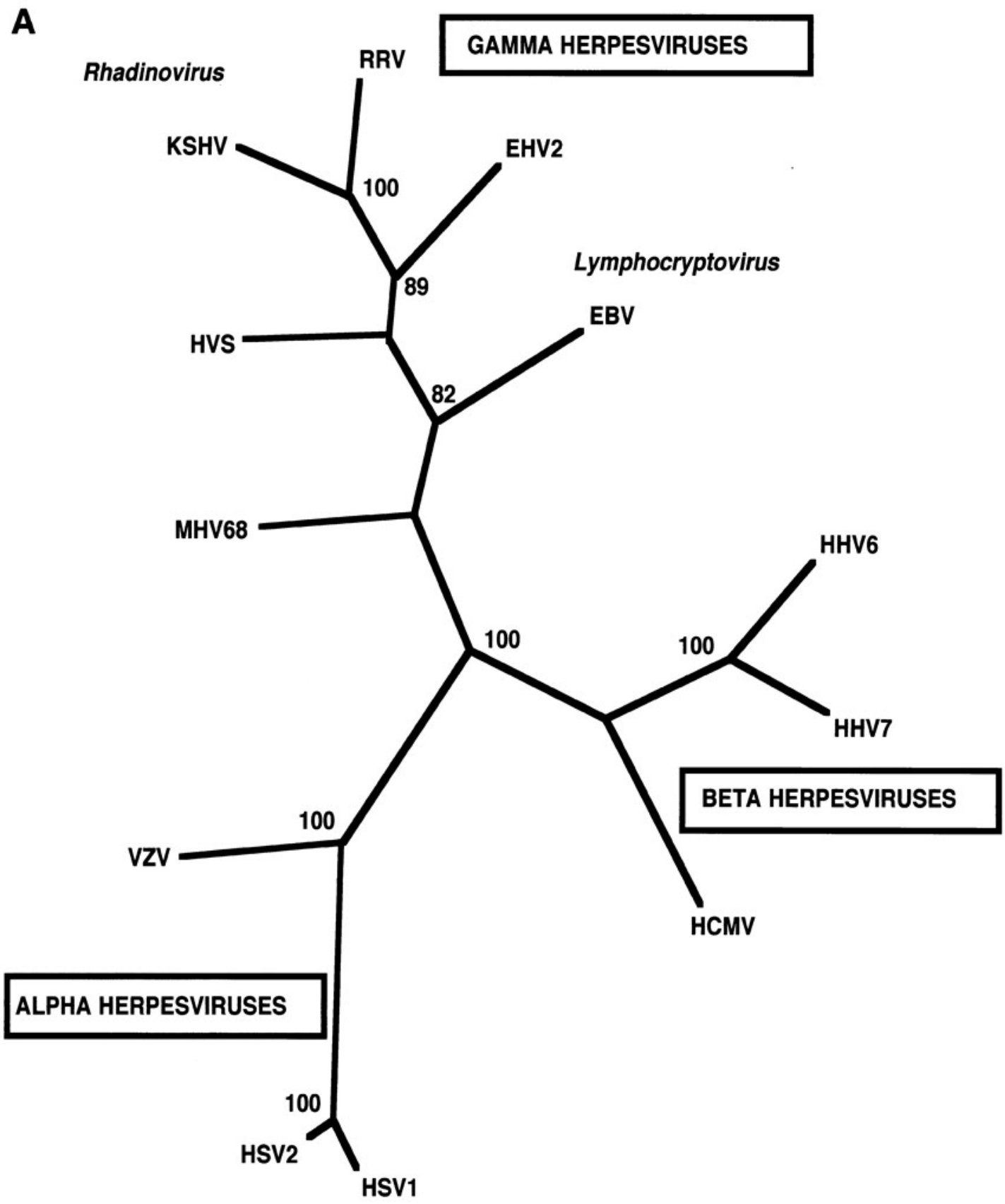


causative agents of oral and genital herpes. Actively replicating HSV causes lesions or blisters, most frequently around the mouth or genitals. Varicella zoster virus (VZV) is the viral agent responsible for chicken pox and shingles (20). Alphaherpesviruses infect cells of the mucoepithelia, but tend to establish latency within neurons (270). $\beta$ herpesviruses include human cytomegalovirus (HCMV) as well as the roselola viruses, human herpesvirus- 6 and 7 (HHV-6 and HHV-7) $(20,92,270)$. HCMV infects a variety of different cell types including fibroblasts, epithelial, smooth muscle, and endothelial cells (reviewed in (292)), yet restricts maintenance of latency to hematopoietic cells (reviewed in (291)). In healthy individuals, HCMV infections may go undetected with no symptoms; however, HCMV can cause congenital birth defects, and - in immunocompromised individuals - infection may cause symptoms resembling mononucleosis ((179) and reviewed in (54)). The gammaherpesviruses are further subdivided into the lymphocryptoviruses, or gamma-1 ( $\mathrm{\gamma} 1)$, and the rhadinoviruses, or gamma-2 ( $\mathrm{Y} 2)$. Epstein Barr virus (EBV) is a y1 lymphocryptovirus that can infect a variety of cell types including monocytes, lymphocytes and epithelia, establishing latency in both monocytes and lymphocytes (reviewed in (117)). EBV infection can lead to mononucleosis, nasopharyngeal carcinoma, Burkitt's lymphoma (reviewed in (171)), the AIDS-related malignancies leiomyosarcoma, Hodgkin's disease, and non-Hodgkin's lymphoma (NHL) (reviewed in $(13,338)$ ). Kaposi's sarcomaassociated herpesvirus (KSHV) is a y2 herpesvirus and the causative agent of Kaposi's sarcoma (KS), as well as two B-cell malignancies, multicentric Castleman's disease (MCD) (297) and primary effusion lymphoma (PEL) 
(reviewed in (46)). KSHV infects endothelial cells, but can also establish latency and replicate in T and B lymphocytes $(123,206,220,221,257)$.

\section{Kaposi's Sarcoma Associated Herpesvirus}

Kaposi's sarcoma-associated herpesvirus (KSHV) is the most recently discovered member of the gammaherpesvirinae subfamily and is specifically the only member of the $\mathrm{Y} 2$ or rhadinoviruses known to infect humans (215). In 1994, Chang and Moore isolated unique DNA sequences from KS tumors that were absent from unaffected tissue in the same patient. These sequences shared similarities to EBV herpesviral DNA (52). These initial fragments allowed the eventual isolation of the entire new herpesvirus genome, named KSHV. The $\mathrm{KSHV}$ genome is approximately $160 \mathrm{~kb}$ and is surrounded by two $\mathrm{G}-\mathrm{C}$ rich terminal repeats $25-35 \mathrm{~kb}$ each. The long unique region (LUR) is approximately $140.5 \mathrm{~kb}$ and contains a region encoding over $80 \mathrm{ORFs}(216,269)$. KSHV infection can lead to transformation of endothelial cells and phenotypic changes that induce the cells to resemble tumor spindle-cells (93). KSHV encodes a number of viral genes, including homologues of cellular genes, that can affect cell signaling, deregulate the cell cycle, and stimulate angiogenesis, leading to the development of malignancies (see Table 1-1 for a summary of their functions) (reviewed in $(13,14,37,280,319)$ ). Saliva largely accounts for KSHV transmission, and unlike most other human herpesviruses, KSHV infection is not universal within the world's population. Infection occurs at increased levels in some ethnic groups and in men who have sex with men. Transmission of KSHV 
Table 1-1. Potential KSHV Genes Involved in Tumorgenesis

Table from (13) and used with permission. 


\begin{tabular}{|l|l|} 
Viral gene & Function \\
\hline K1 & $\begin{array}{l}\text { Signal transduction of receptor binding. } \\
\text { Involved in deregulation of NF-KB. }\end{array}$ \\
\hline K12 (Kaposin) & $\begin{array}{l}\text { Kaposin A is a type II membrane } \\
\text { protein; Kaposin B involved in the } \\
\text { MAPK signaling pathway. }\end{array}$ \\
\hline $\begin{array}{l}\text { VGPCR (viral G-protein coupled } \\
\text { receptor) }\end{array}$ & $\begin{array}{l}\text { Homologue of cellular IL-8 receptor } \\
\text { and binds to CXC and CC chemokines. } \\
\text { It stimulates MAPK pathway and leads } \\
\text { to secretion of VEGF. }\end{array}$ \\
\hline VIL-6 (viral interlukin-6) & $\begin{array}{l}\text { Homologue of cellular IL-6, it supports } \\
\text { cell growth and protects the cells from } \\
\text { undergoing apoptosis. }\end{array}$ \\
\hline K4, K4.1, and K6 & $\begin{array}{l}\text { Viral homologues of cellular CC } \\
\text { chemokins such as RANTES and MIP- } \\
1 \text { a. They induce signal transduction } \\
\text { and enhance angiogenesis. }\end{array}$ \\
\hline $\begin{array}{l}\text { VFLIP (viral FLICE-inhibitory protein or } \\
\text { ORF71) }\end{array}$ & $\begin{array}{l}\text { Homologue of cellular FLIP, it activates } \\
\text { NF-kB pathway and protects cells from } \\
\text { apoptosis. }\end{array}$ \\
\hline $\begin{array}{l}\text { LANA (latency associated nuclear } \\
\text { antigen) }\end{array}$ & $\begin{array}{l}\text { Important in maintenance of viral } \\
\text { latency and binds to viral genome and } \\
\text { a number of cellular factors, such as } \\
\text { p53. }\end{array}$ \\
\hline
\end{tabular}


by organ transplant is also a concern (reviewed in (319)). KSHV exists primarily in its latent form and current studies rely primarily on reactivation of KSHV from latently infected B cell lines (259), which is an artificially induced reactivation of the lytic life cycle, rather than a primary infection. However, even following induction of the lytic transcription program, only $25-30 \%$ of latently infected cells undergo lytic reactivation (49), with a mere 5 to $10 \%$ of induced cells producing progeny virions (259).

$\mathrm{KSHV}$ is the causative agent of $\mathrm{KS}$, described in more detail in the next section, as well as two B cell lymphomas, PEL and MCD (reviewed in (46)). MCD is an uncommon, but highly aggressive lymphoproliferative B cell tumor. KSHV positive MCD constitutes a subset of MCD, called plasmablastic MCD, and displays a large accumulation of plasmablastic cells in the interfollicular region (reviewed in $(41,85))$. PEL is also a B cell lymphoma thought to be clonal in origin, but unlike $\mathrm{KS}$ and $\mathrm{MCD}$, it typically forms pericardial or pleural effusions without a tumor mass (reviewed in (39)). PEL is a form of NHL also known as body cavity-based lymphoma and found frequently in HIV infected individuals. PEL is rare and has a high morbidity rate (reviewed in (45)).

\section{Kaposi's sarcoma}

In 1872, a Hungarian dermatologist, Moritz K. Kaposi, first described Kaposi's sarcoma as "idiopathic multiple pigmented sarcoma of the skin," in the skin of five men (154). KS is a spindle-cell tumor that can present with cutaneous lesions (115). Unlike other common tumors, KS contains multiple cell types, including 
inflammatory cells as well as endothelial cells - the precursors of tumor spindle-cells ((30) and reviewed in (319)). KS lesions can have multiple morphologies, including patch, plaque, and nodular. The patch stage is the earliest phase in the development of cutaneous KS, depicted by bluish red skin spots, and subtle changes that appear histologically in the vicinity around the existing vasculature, with the addition of irregular spaces and newly formed vessels (reviewed in (115)). In the plaque stage, the existing vasculature increases in cellularity, endothelial cells line spaces formed in the patch stage, and cells spindle. In the nodular stage, lesions can appear slightly elevated from the skin, which can be hard, brownish in color, and ulcerative $((97,137)$ and reviewed in (115)).

There are four clinical variants of Kaposi's sarcoma including Classic, Endemic, latrogenic - or transplant-associated, and HIVIAIDS-associated (reviewed in (14)). Classic KS primarily affects Mediterranean and Eastern European elderly men with a median age of 64 (72). KS lesions and nodules appear typically on the feet and hands and can progress up the legs and arms over the years. Classic KS is relatively mild and slow-progressing, with a median survival of years or decades (72).

Discovered in the 1950's in portions of Southern and Central Africa, Endemic KS is a more aggressive form than classic KS (307). In endemic forms of KS, disease nodules or plaques on limbs are common. Patients maintain health until more aggressive lesion growth and dissemination begins. Unlike classic KS, the endemic variant affects children and is one of the leading 
cancers in HIV positive African children $(21,79)$.

latrogenic or transplant-associated, KS affects individuals on immunosuppressive therapy $((89,104,287)$ and reviewed in $(13,14))$. Median time from organ transplant to KS diagnosis is anywhere from 2 months to eight years (reviewed in (13)). latrogenic KS may or may not be associated with cutaneous lesions, but can be aggressive affecting mucosa, visceral organs, and lymph nodes in a large percentage of patients (reviewed in (14)).

HIVIAIDS-associated KS was first described in 1981 when a large number of previously healthy homosexual young men were diagnosed with Pneumocystis carinii pneumonia, in addition to KS, with no obvious cause (96). HIVIAIDSassociated KS is very aggressive and often quickly forms skin lesions on not only the hands and feet, (as is typical in classic KS), but also disseminates throughout the body, including the head, upper body, viscera, organs and lymph nodes. This variant frequently leads to opportunistic infections, due to the defect in cellmediated immunity, which can be life threatening and often fatal $((83,96)$ and reviewed in $(127,128))$.

\section{Rhesus monkey rhadinovirus (RRV)}

Scientists first described Rhesus monkeys rhadinovirus (RRV) when antibodies from a number of monkeys in the New England Regional Primate Research Center (NERPRC) reacted by ELISA to herpesvirus saimiri (HVS), a y2 herpesvirus that infects monkeys (69). Researchers sequenced an approximate $10 \mathrm{~kb}$ piece of DNA from RRV isolate $\mathrm{H} 26-95$, which showed strong similarity 
between HVS and other rhadinoviruses. The genes encoding glycoprotein B and DNA polymerase showed sequence identity demonstrating relatedness closer to KSHV than to any other herpesvirus, at $65 \%$ and $67 \%$, respectively (69). Unlike KSHV, which is primarily a latent herpesvirus producing low viral titers $(94,259), R R V$ is a robust lytic virus replicating to high titers in cultured rhesus monkey fibroblasts (RhF) (69).

Not long after the first isolation and early description of RRV H26-95 (69), researchers isolated a closely related strain of RRV from the Oregon Regional Primate Research Center (ORPRC). Sequencing of the long unique region (LUR) (the non-terminal repeated portion of the genome) of RRV isolate 17577 confirmed a genome 131,365 bp long with a $\mathrm{G}+\mathrm{C}$ content of $52.5 \%$, coding for over 79 open reading frames (ORFs), and a similar genomic organization to KSHV. Of these ORFs, 67 have similar genes found in both HVS and KSHV (281). Comparison of the initial $10 \mathrm{~kb}$ genomic segment in RRV H26-95 (69) to the corresponding RRV 17577 segment show a greater than 99\% identity between the two isolates of RRV, indicating they are different strains of the same virus (281). The RRV 17577 isolate was linked to simian immunodeficiency virus (SIV)-associated lymphoprolferative disorder (LPD) (337).

Subsequent research on RRV H26-95 shows the LUR of RRV H26-95 is 130,733 bp and contains 84 ORFs. Except for K3, K5, K7, and K12, all KSHV ORFs have homologs in RRV H26-95, share similar genomic organization, and have similar lytic viral replication gene expression profiles (Figure 1-2) (70). Comparison of the RRV H26-95 isolate to RRV 17577 indicates 83 out of 84 
FIGURE 1-2. Alignment of ORFs of KSHV and RRV26-95. Conserved ORFs in KSHV and RRV26-95 are indicated and different colors signify conservation in herpesvirus subfamilies or subgroups. Figure from (12) and used with permission. 

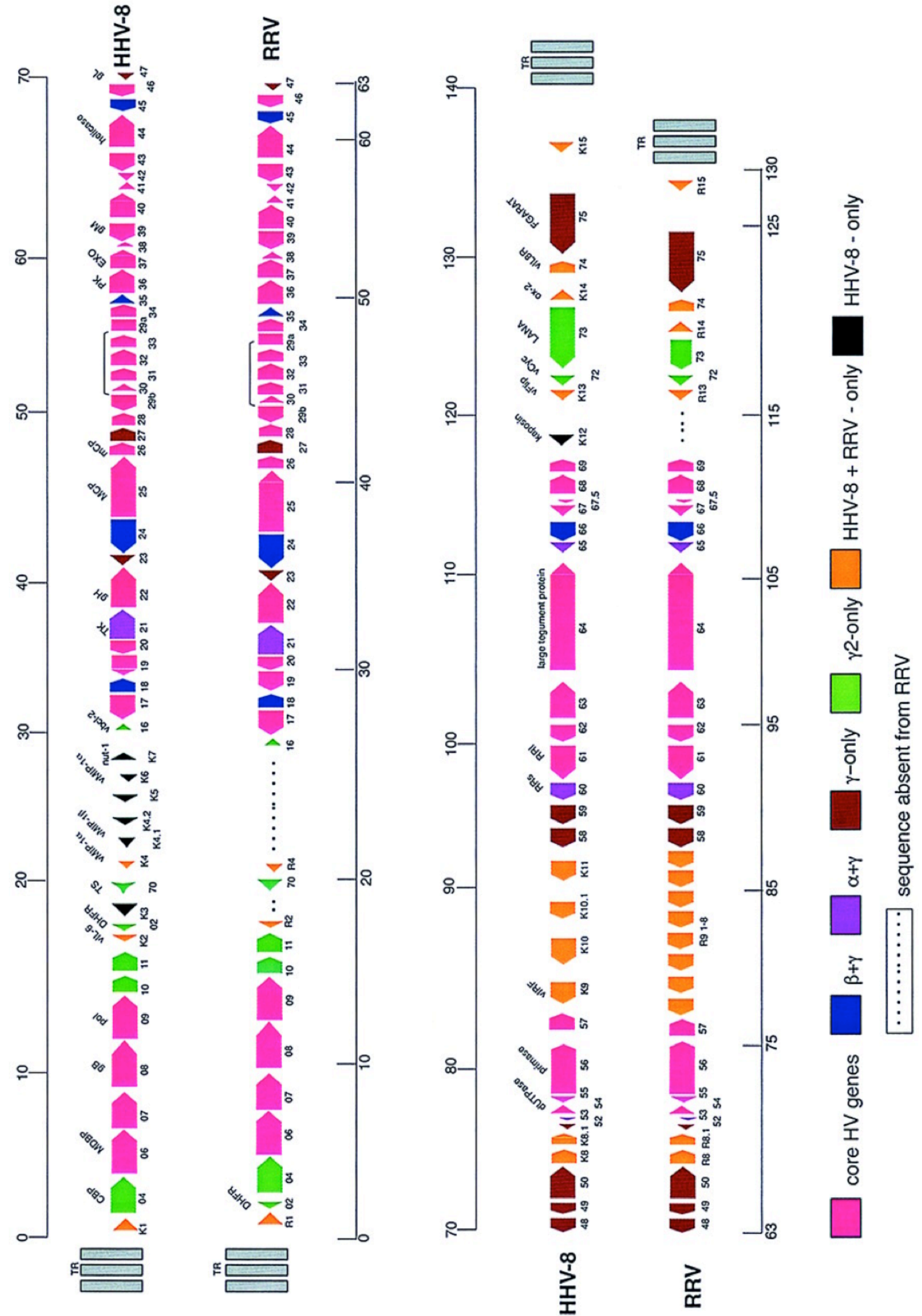
ORFs contain sequence similarity (12).

\section{Herpesvirus Structure}

All herpesviruses share a common virion structure that involves a complex coordination and layering of an excess of 30 viral proteins, in addition to a number of cellular proteins. The linear double-stranded viral DNA is at the innermost core of the herpes virion. An icosahedral capsid surrounds this DNA $((239,349)$ and reviewed in $(36,263))$. The viral tegument - a thick layer composed of viral and cellular proteins - surrounds the capsid. The outermost layer is a host cell derived envelope studded with many viral glycoproteins, which aid in attachment to host cells and function in secondary envelopment, in addition to cellular proteins (Figure 1-4) $((55,92,356)$ and reviewed in (209)). The herpesvirus capsid shell comprises 162 capsomers, 150 hexavalent capsomers (hexons), and 12 pentavalent capsomers (pentons). The capsomers lie on a $\mathrm{T}=$ 16 icosahedral lattice, with pentons located at the vertices and hexons occupying the capsid faces and edges ((92) and (reviewed in $(36,133))$ (Figure 1-3). There are three types of herpesvirus capsid species produced: A, B, and C capsids $(238,299,349,356,358)$.

\section{Herpesvirus capsid}

In KSHV and RRV, six capsid proteins compose the capsid: major capsid protein (MCP encoded by ORF25), small capsomer interacting protein (SCIP/ORF65), two triplex proteins - TRI-1 (ORF62), TRI-2 (ORF26), the portal protein 
Figure 1-3. Herpesvirus structure. Herpesviruses have a linear dsDNA core, surrounded by an icosahedral capsid, an inner and outer tegument, and a glycoprotein-studded envelope. Image credit: collaboration with Mary A. Maxfield and used with permission. 


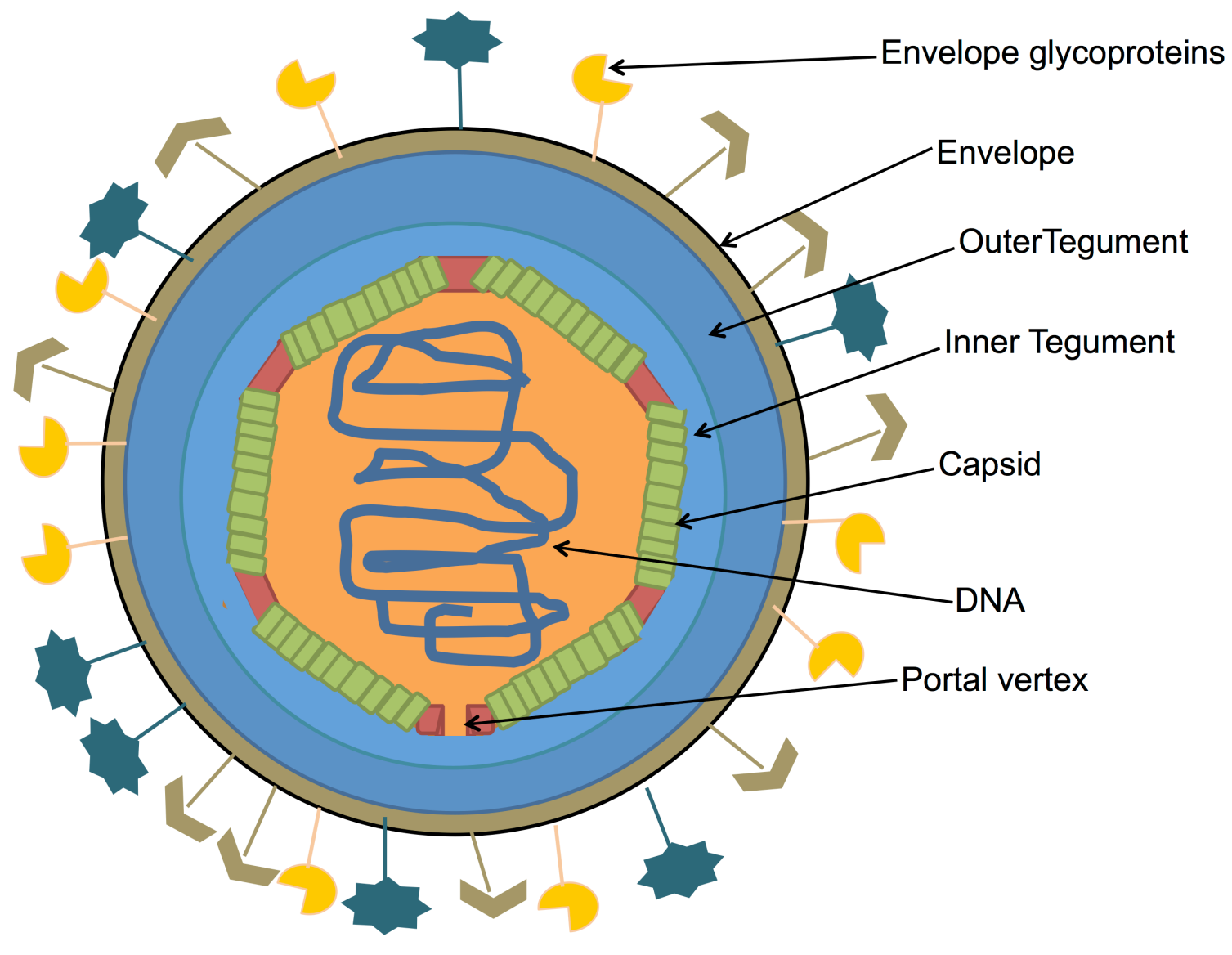


(PORT/ORF43), and the portal capping protein (PCP/ORF19) $(68,226,239$, 358). 955 copies of MCP make up each icosahedrial capsid - 150 hexons each with 6 copies of MCP and 11 pentons with 5 copies each. The remaining penton contains the portal protein, ORF43, $((68,228-230,349)$ and reviewed in $(36))$. These capsid proteins are conserved within the herpesviruses and have homologs in HSV-1: VP5 (MCP), VP19c (TRI-1), VP23 (TRI-2), VP26 (SCIP), pUL6 (PORT), and pUL25 (PCP) (encoded by UL19, UL38, UL18, UL35, UL6, and UL25 respectively) (reviewed in (36)). The C capsids are identical in capsid protein composition to A capsids; however, they contain the linear DNA genome. B capsids are a unique intermediate species that contain a scaffold protein, ORF17.5 in KSHV/RRV and VP22a in HSV-1, and lack DNA. Experiments with alphaherpesviruses indicate that A and B capsids are essentially dead-end products and cannot mature further (reviewed in $(36,133,263)$ ).

\section{The herpesvirus tegument}

The herpesvirus tegument layer lies between the envelope and the capsid and comprises both cellular and viral proteins (reviewed in (208-211)). Proteomic analysis on the composition of several herpesviruses including EBV, HCMV, HSV-1, KSHV, RRV, and murine gammaherpesvirus 68 (MHV-68) has identified virion components specific to each virus as well as their location within the particle $(17,28,142,158,184,239,325,358)$.

Studies examining the components of the herpesvirus virions indicate that tegument proteins make up over half of the total number of viral proteins 
(reviewed in (149)). Recent studies have established that the number of different tegument proteins present in each herpesvirus is as follows: HSV-1 has 26 ((184) and (reviewed in (209)), human cytomegalovirus (HCMV) has 14 (28), EBV has 23 (325), MHV-68 has at least $10(28,327)$, KSHV has $11(23,267$, 358) and RRV has 17 (239). Until recently, investigators believed proteins within the tegument were an amorphous collection; however, cryoelectron microscopy (cryo-EM) studies with human cytomegalovirus (HCMV) (55), MHV-68 (62), and HSV-1 (356) have indicated the presence of ordered tegument structures involving at least a subset of the tegument proteins. This ordering is significant enough to be further divided into "inner" and "outer" tegument layers, which are seen with cryo-electron tomography (Figure 1-4) (62). While the research does not strictly define the terms "inner" and "outer," the former generally refers to tegument proteins more tightly associated with the capsid and more resistant to Triton X-100 detergent treatment (336). Large tegument protein (LTP), ORF64 (VP1/2 in HSV-1 encoded by UL36), exists in all herpesviruses and researchers commonly refer to it as an inner tegument protein. LTP plays an important role in the protein-protein interactions of the tegument $(40,267,330)$. Studies have shown that tegument proteins function in at least four crucial roles in viral replication including: 1) transcytosis of herpesvirus capsid following viral entry of the host cell toward the nucleus $(113,187,295,350), 2)$ modulation of the host cell environment during the immediate-early phase of infection (294), including shut off of host gene expression $(289,301,302), 3)$ immune system evasion 
Figure 1-4. Cryo-electron tomogram reconstruction of the MHV-68 virion. The structure of the virion as well as interactions between the herpesvirus capsid, tegument, and envelope. Image from (62) and used with permission. 


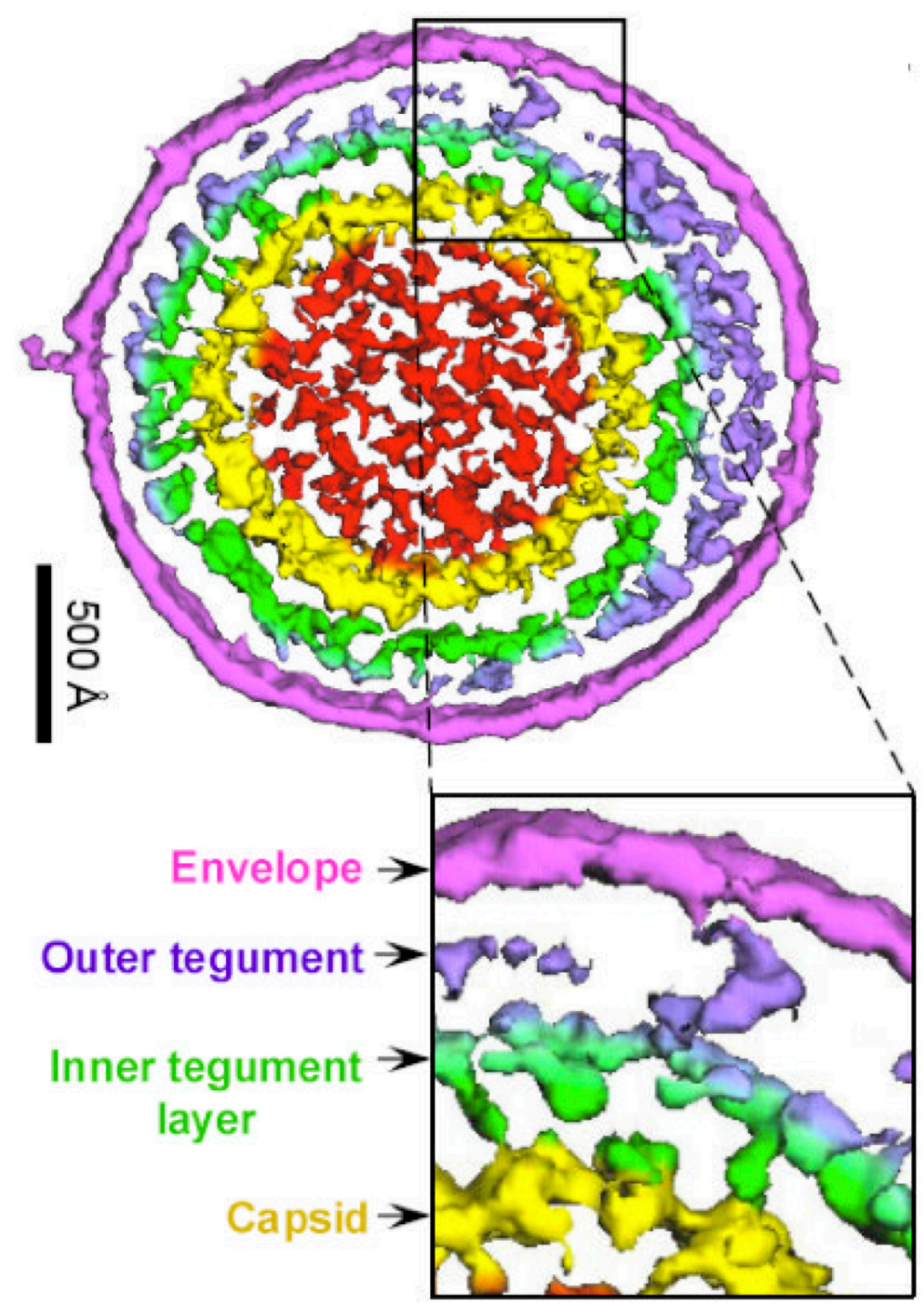


$(102,147,178,180,276,362)$ including interaction with cellular proteins to promote viral replication $(82,141,237)$, and 4) assembly and egress of herpesvirus virions $((27,57,120,277)$ and reviewed in $(209-211))$. Investigators have not yet defined the functions of the vast majority of the tegument proteins within gammaherpesviruses, though several of the proteins have presumed functions based on varying degrees of homology to other herpesviral tegument proteins.

A subset of tegument proteins is present only in the gammaherpesviruses and their functions remain largely unknown. Of the 17 tegument proteins in RRV, 5 are found only within the gammaperpesvirinae subfamily. They are ORFs 27 , $45,49,52$, and 75 (239), and I will discuss them separately at the end of this section.

\section{Herpesvirus replication}

As mentioned above, all members of the Herpesviridae family have two phases of viral replication programs: latent and lytic. In the latent stage, cells express only a small number of the viral genes necessary for maintenance of the viral genome (359). During latency, in KSHV, the viral protein, latency associated nuclear antigen (LANA), tethers the circularized viral episome to the host chromatin (18). In contrast, in the lytic stage, the cell actively transcribes the linear viral DNA and expresses almost all of its genes, producing progeny virions. Latency is indispensable, as it allows herpesviruses to evade the immune response, persist indefinitely within host cells, and-in the case of 
gammaherpesvirus infection-to possibly lead to transformation events that induce malignancies (reviewed in $(332,345)$ ). While herpesvirus lytic reactivation in latently infected cells can be induced with addition of drugs like histone deacetylase (HDAC) inhibitors or phorbol esters, causes of spontaneous reactivation are not yet well understood.

An overview of the herpesvirus lytic replication cycle is shown in Figure 15. Primary (de novo) infection begins when virions attach to the cell membrane mediated by viral glycoproteins engaging with various host cell receptors. Viral glycoproteins conserved across the herpesviruses include $\mathrm{gB}, \mathrm{gM}, \mathrm{gN}, \mathrm{gH}$, and gL (reviewed in $(50,125,167))$. For KSHV, cellular receptors include ephrin A2 (EphA2), heparin sulfate, integrins - specifically $\alpha 3 \beta 1, \alpha v \beta 5, \alpha 8 \beta 1$, and $\alpha$ llbv $\beta 3$, the cysteine/glutamate transporter (xCT), and dendritic cell-specific intercellular adhesion molecule-3 grabbing nonintegrin (DC-SIGN) $((6,122,135,148,161$, $252,257,258)$ and reviewed in (48)). RRV gB shares sequence homology to its homolog in $\mathrm{KSHV}$, but, in contrast, to KSHV, RRV gB does not engage with integrins $\alpha 3 \beta 1$ or $\alpha v \beta 5$ and does not have identified receptor(s) for mediating entry (107) and reviewed in (352)). RRV enters the cell by different mechanisms, depending on cell type, including clatharin- and caveolin- mediated endocytosis and macropinocytosis (reviewed in $(48,50)$ ). RRV enters fibroblasts, for example, through clathrin-mediated endocytosis (353). Upon fusion of the viral envelope with the cellular plasma membrane or endocytic vesicle, the capsid is released into the cytoplasm and loses the majority of tegument proteins from the virion $(23,113,195)$. Capsids travel along microtubules by the minus-end 
Figure 1-5. Lytic replication cycle of herpesviruses. Representation of the herpesvirus replication cycle, including virus entry and dissociation of tegument, transport of incoming capsids to the nuclear pore, and release of viral DNA into the nucleus, where transcription occurs in a cascade-like fashion and DNA replication occurs. Following nuclear egress, DNA filled C capsids undergo tegumentation, secondary envelopment, and egress. Image from (208) and used with permission. 


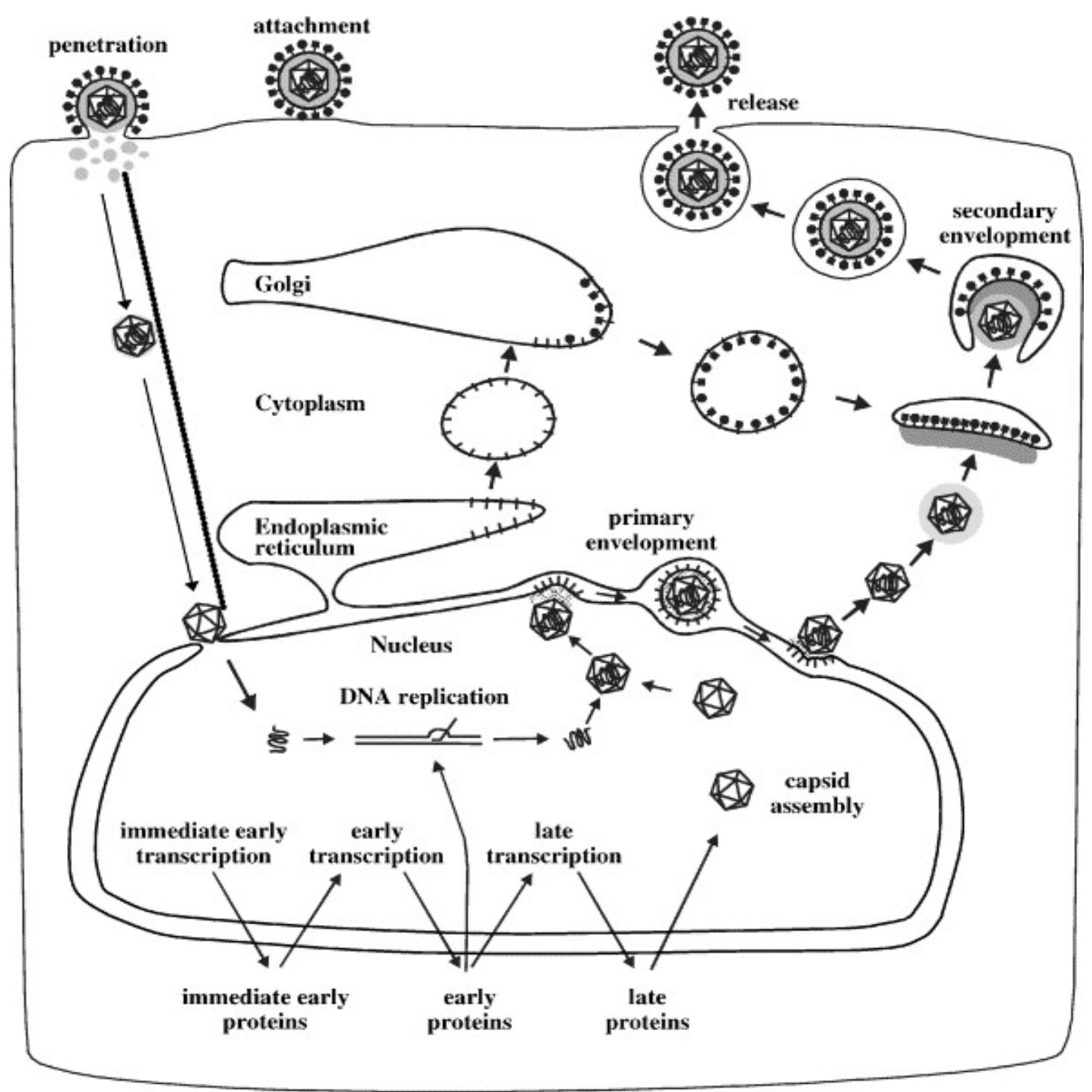


(retrograde), directed dynein/dynactin motor protein complex to the nucleus $((76,225)$ and reviewed in $(74,116))$. In HSV, capsids partially tegumented with VP1/2 and UL37 proteins then bind to the host nuclear pore complex (NPC), where the viral DNA is "uncoated" or released into the nucleus $(60,241,295)$. The linear genome then converts into a covalently closed circular form required for viral replication (106).

Researchers propose that DNA replication initially precedes by a theta type mechanism and, by a yet undetermined mechanism, switches to the rolling circle mode of replication, generating long head-to-tail concatemers. Concatemers are cleaved into individual herpesvirus genomes upon packaging into viral capsids aided by the terminase protein complex $(103,222)$. Transcription of herpesvirus genes, including latent, immediate-early, early, and late genes, takes place within the nucleus. Immediately-early genes are the first viral genes expressed following the initiation of lytic replication of latently infected cells, whether spontaneous or induced, with phorbal esters like tetradecanoyl phorbol acetate (TPA) or histone deacteylase (HDAC) inhibitor, or following primary (de novo) infection, and do not require new viral protein synthesis. These genes often encode regulatory proteins critical for the viral life cycle. Early genes often play a role in regulation of gene expression and require viral protein synthesis of immediate early genes. Late genes require DNA replication and often participate in the major structural components of the herpesvirus particle, including formation of the viral icosahedral capsid and many, but not all, of the tegument proteins $((73,144,185,248,359)$ and reviewed in (208)). Following 
translation of viral mRNAs on ribosomes in the cytoplasm, all capsid proteins are transported back into the nucleus either by their own nuclear localization signal (NLS) or by binding to another protein with an NLS $(165,250,264)$.

More is known about HSV-1 in the area of capsid maturation and, in the following section, I will utilize the HSV-1 nomenclature. The B capsid core includes UL26 encoded proteins VP21 and VP24, as well as the UL26.5 encoded protein VP22a. UL26 cleaves itself to generate the capsid proteins VP24 and VP21 and also cleaves the UL26.5 gene product, generating VP22a. VP22a is the most abundant protein, forming the inner scaffolding core, which aids in assembly of the outer capsid shell structure $(66,181,249)$. Cleavage from VP24 upon DNA encapsidation removes VP21 and VP22a from B capsids to make room for the linear genome, whereas VP24 remains (110). Studies in insect cells with purified capsid proteins have shown that mature, icosahedral capsids can be assembled from the following four HSV-1 proteins: VP5, VP19C, VP23 and preVP22a (232-234).

Interaction between the scaffold protein precursor, pre-VP22a, with VP5 (major capsid protein) appears to initiate in the cytoplasm, whereby they enter the nucleus via the pre-VP22a NLS and begin assembly into hexons and pentons ((339) and reviewed in (133)). The HSV-1 UL26 gene encodes the virus protease essential for DNA packaging, capsid maturation, and virus growth (105). The mature capsid is formed from a fragile, porous, spherical intermediate called the procapsid, which has the same protein composition as the mature capsid, except that pre-VP22a is not cleaved in the procapsid $(233,236)$. Cleavage of the 
scaffold protein by the viral protease causes a cascade of structural changes that result in transformation of the procapsid into an icosahedral B capsid ((315) and reviewed in (133)).

The proteins encoded by UL17 and UL32 (ORFs 32 and 68 in KSHV/RRV) localize capsid protein precursors important in forming procapsids $(173,305)$. The capsid-associated protein encoded by UL25 (ORF19) is present on $A, B, C$ capsids as well as virions $(231,285)$. UL25 forms a heterodimer with UL17 and attaches to the exterior capsid vertices $(310,316)$. Additionally, UL25 reportedly interacts with the triplex protein VP19c, as well as the major capsid protein VP5 $(240,316)$. Accumulation of UL25 protein may reinforce the capsid to prevent loss of DNA, similar to the role of accessory proteins in dsDNA bacteriophages $(203,231)$. Studies have also recently described a role for UL25 early in infection, involving the uncoating of the viral genome (254), as well as the requirement of UL25 for attachment of the tegument to the capsid through its interaction with the large tegument protein UL36 (59).

The herpesvirus DNA encapsidation process is still understood only in general terms and primarily from studies on the alphaherperpesviruses pseudorabies virus (PrV) and HSV-1; however, it resembles packaging observed in dsDNA bacteriophages, such as P22, T7, and $\lambda$. Studies suggest DNA packaging in KSHV and RRV occurs by the same mechanisms as in HSV-1, even though -unlike DNA in phages-the KSHV portal protein is internally localized (68). HSV-1 and dsDNA bacteriophages share similar functions, such as capsid portal structure, proteins that recognize the ends of viral DNA, and an 
endonuclease plus a DNA translocase (terminase complex) $(26,43)$. Moreover, the major core protein of the precursor capsid is also expelled during packaging, as with phage assembly $(22,322)$.

In HSV-1, DNA enters the capsid via the portal vertex, which consists of 12 copies of the UL6 (ORF43) protein. The portal is located at only one of the 12capsid vertices and is the docking site for the packaging proteins, such as terminase, the protein responsible for cleavage of monomeric units from concatemeric DNA and ATP-dependent translocation of DNA into the capsid (26, 42, 43, 322). Portal proteins are integral capsid components in both procapsids and mature capsids, while terminase proteins are only associated with procapsids and are not present in mature capsids or virions (76). Studies have found that the interaction of the scaffold protein and the portal proteins is essential for incorporation of the portal into the capsid and, additionally, that the portal proteins need to be present when the assembly reaction begins (231).

In addition to the UL6 portal protein, DNA packaging involves six HSV-1 proteins. These proteins are the products of genes UL15, UL17, UL25, UL28, UL32 and UL33 $((7,33,53)$ and reviewed in $(132,133,173,203,246,253$, 309)). Infection with mutants lacking any one of these seven genes (except for UL25) results in uncleaved concatameric DNA and accumulation of B capsids (235). Neither cleavage nor insertion of DNA into the capsid requires UL25; its role is in maintenance of the viral genome after packaging. Disruption of the UL25 gene results in nuclear accumulation of both $A$ and $B$ capsids in addition to unpackaged, genome-length DNA (203). Studies suggest the HSV-1 terminase 
complex is composed of UL15, UL28 and UL33 subunits $(2,333,343)$, which assemble in the cytoplasm and are then transported into the nucleus utilizing a NLS within UL15 (348).

Nuclear egress of DNA filled C capsids or A and B capsids, occursaccording to wide consensus-in what is coined the primary envelopment, deenvelopment, and re-envelopment pathway ((293) and reviewed in (213)). Of note, investigators continue to debate the method of nucleocapsid egress from the nucleus into the cytoplasm $(176,212,334)$. Leuzinger, et al. proposed an alternate pathway not entirely unlike those previously proposed (92), where capsids bud into the inner nuclear membrane (INM), obtain an envelope, and subsequently travel through the rough endoplasmic reticulum (RER) cisternae, prior to being sorted and packaged within transport vacuoles to the cell surface (176). A second alternative involves capsids gaining direct access to the cytoplasm by migrating though impaired nuclear pores $(176,334)$ (Figure 1-6). These hypotheses have taken criticism, most vocally from Thomas C. Mettenleiter, who has written extensively on the primary envelopment, deenvelopment, and re-envelopment pathway (reviewed in (208-213)). Concerns include the published evidence that some proteins present in primary enveloped particles within the INM such as the tegument proteins UL31 and UL34 in HSV (ORF69 and ORF67 in KSHV and RRV) are not present in mature virions (101, 163, 260). Additionally, nuclear pore size typically restricts direct passage for substances larger than $36 \mathrm{~nm}$ (243). While the integrity of the nucleus changes during the course of infection, possibly widening the pore size to accommodate 
Figure 1-6. Diagram of the four models for exit of herpesvirus capsids from the nucleus. (A) Single envelopment model entailing capsid budding at INM and transport via lumenal pathway. (B) Capsid movement into cytoplasm through dilated nuclear pores. (C) Dual envelopment model entailing vesicle-mediated nuclear egress of capsids (primary envelopment-de-envelopment, and reenvelopment). (D) Nuclear escape of capsids after nuclear envelope breakdown (not officially proposed as an alternative, but possible). Image from (213) and used with permission. 


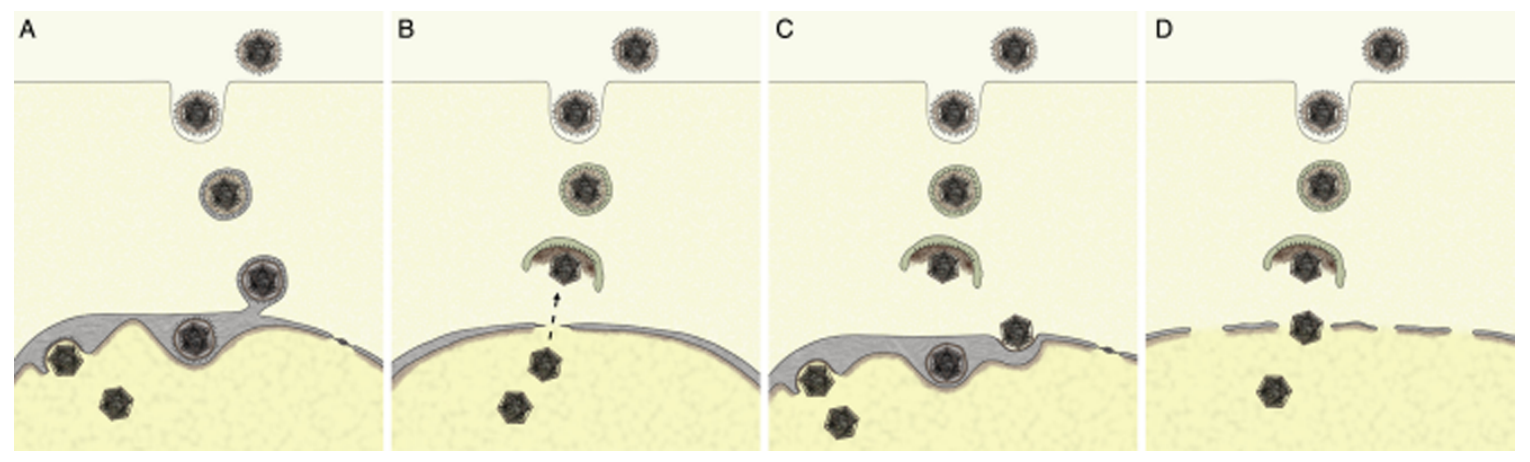


a $100-200 \mathrm{~nm}$ herpesvirus particle, the nuclear pore appears to remain intact until late in infection (reviewed in (213)). In this thesis, I will reference the envelopment, de-envelopment, and re-envelopment pathway (293), when discussing the latter maturation steps.

The proteins, interactions, and even the particular stimulus that triggers capsid egress from the nucleus into the cytoplasm to further mature are not thoroughly understood. Researchers do know that the heterodimeric complex composed of proteins encoded by UL31 (pUL31) and UL34 (pUL34) (ORF69 and ORF66, respectively, in KSHV and RRV), are conserved across the herpesviruses (272) and are critical for formation of primary enveloped virions (reviewed in (143)). Phosphorylation of laminins by cellular and viral kinases including HSV pUL13 and its homologs in other herpesviruses, (ORF36 in KSHV and RRV) (199) softens the nuclear lamina, to allow for cargo transport through the nuclear membrane (reviewed in $(193,217,219)$ ). In PrV and HSV, infection leads to accumulation of myosin $\mathrm{V}$, in addition to inducing nuclear actin, which may play a role in capsid movement in, and egress from, the nucleus (90). Other viral proteins in addition to pUL31 and pUL34 may be present and involved in nuclear egress, but difficulty in consistently isolating large quantities of primary enveloped virions (PEVs) between the nuclear membranes has made this a difficult challenge. Of note, William W. Newcomb, a researcher at the University of Virginia, has developed a technique to isolate these HSV-1 PEVs and is optimistic about the success in defining their composition (personal correspondence, February 2014). 
Following fusion with the outer nuclear membrane (ONM), capsids are released into the cytoplasm. At these latter points in virion maturation, capsids now need to traverse through the cytoplasm, and obtain their full repertoire of tegument proteins and envelope prior to release. The kinesin family of motor proteins utilizes the plus-end directed microtubule network to mediate capsid movement throughout the cytoplasm (reviewed in $(75,188,251)$ ). Interestingly, research shows that KSHV gammaherpsvirus specific tegument protein ORF45 mediates docking of the herpesvirus capsid prior to secondary envelopment onto the KIF3A subunit of the kinesin-2 motor protein for movement to the cell periphery (277). Current data does not fully explain the timing of addition, specific order, and complex network of protein-protein interactions that make up the tegument layer, but this is an active and exciting area of exploration. Several studies have investigated the role of specific tegument proteins, within a specific herpesvirus (including my own manuscript and this thesis) (reviewed in (119, $143,149,160,211-213)$ ), and while critical to advancing our understanding of this complex and multifaceted layer of the virion, none convey a complete picture or fully elucidate the details of this process. Research on CMV shows that many glycoproteins and tegument proteins aggregate to a perinuclear assembly-like structure, that could be the site of assembly and egress $(64,65,273)$, but it is not present during lytic infection with all herpesviruses. Data reveals that capsids travel in the cytoplasm, likely obtaining some initial tegument prior to secondary (final) envelopment, whereby the partially tegumented capsid obtains the bulk of the tegument proteins and the final envelope from vesicles (possibly Golgi 
derived or endosomes), already studded with viral glycoproteins (reviewed in $(209,211))$. The consensus in the field is that secondary envelopment occurs as the result of a still poorly understood triggering event between viral tegument proteins and glycoproteins present on vesicles that become the viral envelope (reviewed in $(126,213))$. In KSHV, eight glycoproteins were identified as part of the virion (gB, gp35/37, $\mathrm{gH}, \mathrm{pORF} 28, \mathrm{gM}, \mathrm{gL}$, and $\mathrm{gN}$ ) (358) and 9 in $\mathrm{RRV}$ (ORF4, gB, R8.1, gH, ORF28, gM, gL, gN, and ORF58) (239).

Multiple studies report several herpesvirus glycoproteins including gB, gM, $\mathrm{gK}, \mathrm{gE} / \mathrm{gl}, \mathrm{gD}$, and possibly gH localize to the trans-Golgi network for HSV-1 and other herpesvirus family members $(9-11,35,61,95,118,140,202,214,273$, $311,364,365)$, although $\mathrm{gB}$ and - to a lesser degree - gL, did not completely colocalize with the TGN46 marker (318). In HSV-1 the glycoprotein pUL49 (gN in KSHV and RRV) binds to pUL48 (151). pUL48 in turn interacts with the cytoplasmic tail of $\mathrm{gH}$ and $\mathrm{gD}$ (56). Studies show pUL49 of PrV interacts with the cytoplasmic tails of $\mathrm{gE}$ and $\mathrm{gM}(100)$.

During secondary envelopment, tegument proteins engage with glycoproteins leading vesicles to surround the capsids, wrap entirely around the particle, and form an envelope (reviewed in $(126,213)$ ). Hollinshead, et al., recently proposed an alternative mechanism for secondary envelopment, where endocytosis from the plasma membrane formed tubules that then wrapped around HSV-1 capsids in the cytoplasm (131) (Figure 1-7). Evidence that capsids did not colocalize with TGN46, a trans-Golgi network marker, or markers for late endosomal vesicles provides additional support for this method of secondary 
envelopment (131). Of note, in preliminary data from our own laboratory, we detected no colocalization of TGN46 and RRV capsid protein SCIP (ORF65) in the cytoplasm (Loftus and Kedes, unpublished data).

Egress from the cell occurs with enveloped virions inside vesicles transported to the plasma membrane. The plasma membrane fuses with the vesicle and mature virions are released from the cell (reviewed in $(209,211$, 213)). Researchers know little about the viral protein interactions required during egress. Studies implicate PrV pUL20 and gK in egress (reviewed in (208)), whereas in HSV, the pUL20/gK complex apparently functions prior to egress $(166,205)$. Following egress from the cells, mature virions release into the extracellular medium. However, often herpesviruses remain associated with the plasma membrane following egress mediated partly by bone marrow stromal antigen 2 (BST2). BST2, also called tetherin, is a type II glycosylated membrane protein that localizes to lipid rafts (77). Self-association of tetherin molecules between the cellular plasma membrane and those embedded in the viral envelope tether enveloped viruses-including herpesesviruses, retroviruses, and filoviruses_to the plasma membrane following cellular egress $(146,150,271$, 324, 351). However, viruses often encode proteins, including KSHV K5 and HIV$1 \mathrm{Vpu}$, which allow for sidestepping of this anti-viral function of the innate immune system $(78,192,227,244,279,323)$. Following egress, these virions can spread in two different ways. First, the virion may detach from the cellular plasma membrane and spread through the environment to attach to another cell. Alternately, direct cell-to-cell contact from an infected cell to another cell may 
Figure 1-7. Model for virus envelopment via endocytosis. (1) Viral glycoproteins are processed in the Golgi (GA)/trans-Golgi (TGN) and exported to the cell surface. (2) The plasma membrane (PM), containing glycoproteins, is endocytosed and transported through the early endosome (EE) to produce tubules. (3) Tubules containing glycoproteins wrap around capsids, forming virions with a double membrane. These tubules eventually fuse with the plasma membrane releasing enveloped virions. Image from (131), an open-access article. 


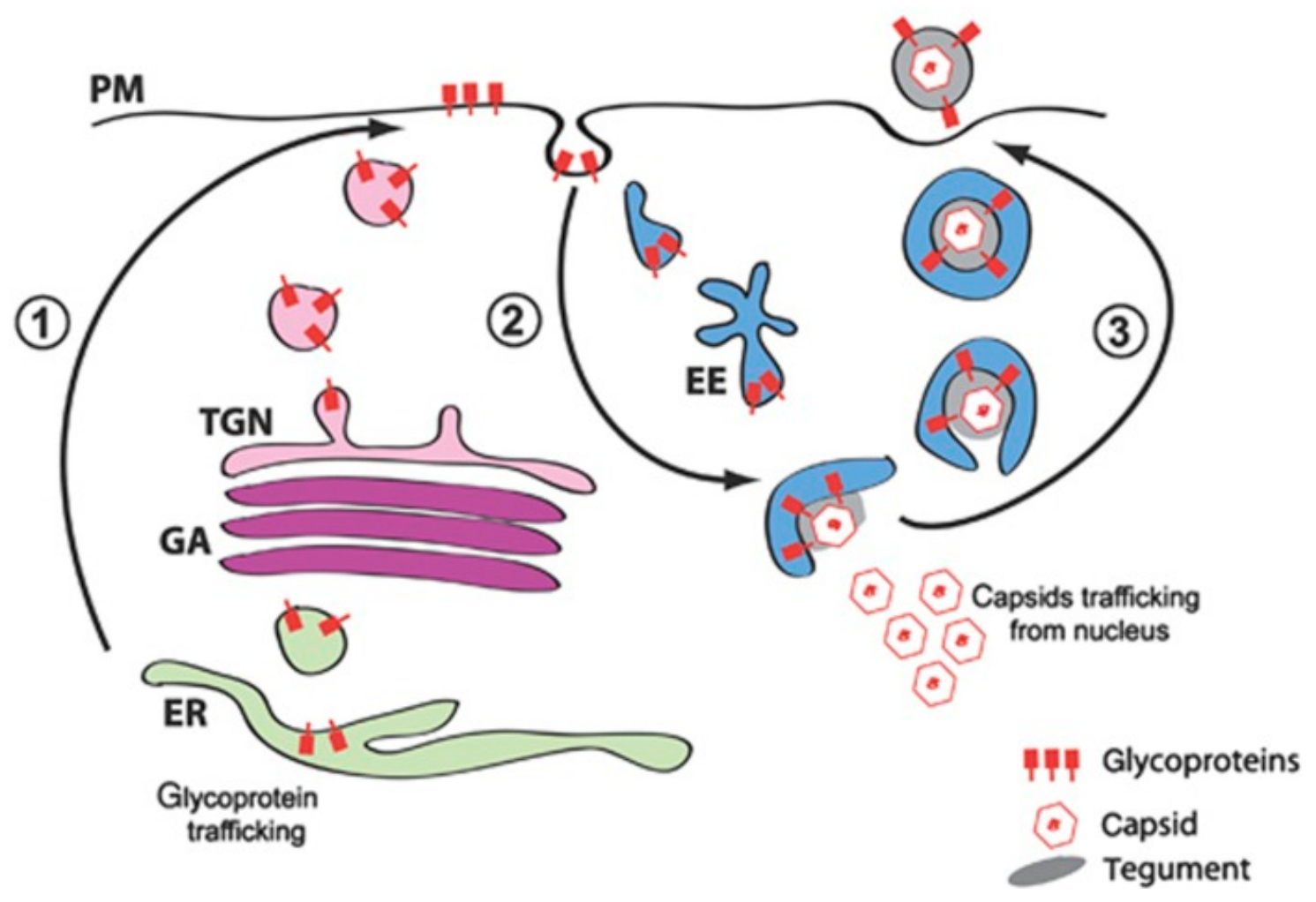


allow for an increase in efficiency of horizontal spread and new infection. Herpesviruses utilize both methods of spread $((218,354)$ and reviewed in $(288))$.

\section{Gammaherpesvirus specific tegument proteins}

Our laboratory has taken a particular interest in the proteins specific to the gammaherpesvirus subfamily as they might play important roles in the tumorigenic potential of the gammaherpesviruses. Below I have detailed what is currently known about ORF52 and I have also included information on the other gammaherpesvirus specific tegument proteins. Several published reports on these gamma-specific tegument proteins investigate their function in MHV-68. MHV-68 is a $\mathrm{Y}-2$ herpesvirus (like KSHV and RRV) that naturally infects mice and thus allows for investigation in both in vivo and in vitro model systems (327).

\section{Gammaherpesvirus ORF52}

ORF52 is a highly abundant, gammaherpesvirus specific protein component of the virion tegument in gammaherpesviruses, including RRV (239), MHV-68 (28), $\operatorname{KSHV}(23,358)$, and EBV (142) (see Figure 1-8 for alignment). In RRV, like MHV-68, ORF52 closely associates with the virion capsid $(27,239)$ and researchers consider it an inner tegument protein. We submitted for publication the first functional study of RRV ORF52 and Chapter 2 of this thesis encompasses this work, focusing on the role of RRV ORF52 during lytic replication. Recent reports have provided evidence for the role of MHV-68 ORF52 $(24,28,330)$, and investigations have most recently been extended to 
the EBV ORF52 homolog, BLRF2 (82). Our work, however, is the first study of a primate rhadinovirus $(\mathrm{Y}-2)$, the closest homolog to $\mathrm{KSHV}$.

\section{MHV-68 ORF52}

In MHV-68, ORF52 is 135 amino acids, with $41 \%$ identity and $63 \%$ similarity to RRV ORF52 and it is a true late gene, expressed after viral DNA replication (5, $84,194)$. In 2005 , by utilizing signature tagged mutagenesis and MHV-68 cloned into a bacterial artificial chromosome (BAC), Song, et al. were able to identify 41 genes essential for in vitro growth; one of these is ORF52 (296). Subsequent work in 2007 by Bortz and colleagues found that MHV-68 ORF52 and the virion capsid exhibit close association with another gammaherpesvirus specific tegument protein, ORF45. By tagging ORF52 with either enhanced green fluorescent protein or a FLAG epitope, researchers observed ORF52 localizing to the cytoplasm. When researches transfected a recombinant MHV-68 BAC with a nonsense (stop) mutation incorporated at ORF52 (ORF52STOP BAC/52S) into 293T cells, the null virus still managed to replicate its DNA; however, it was unable to produce infectious virions that could subsequently infect naïve cells. Furthermore, thin section transmission electron microscopy (TEM) of transfected cells showed an accumulation of immature, partially tegumented capsids in the cytoplasm and an absence of extracellular virions from those cells transfected with the ORF52-null virus (27). In an effort to gain additional insight into MHV-68 ORF52, Benach and colleagues solved the crystal structure at $2.1 \mathrm{~A}$ resolution (24). In addition to the structural data, coimmunoprecipitation and fluorescence 
Figure 1-8. Alignment of ORF52 in RRV, KSHV, MHV-68, and EBV. Strictly conserved residues are highlighted in red and conservatively conserved residues in yellow. Alignment done using CLUSTAL $O$ (1.2.1) software (http://www.ebi.ac.uk/Tools/msa/clustalo/). 


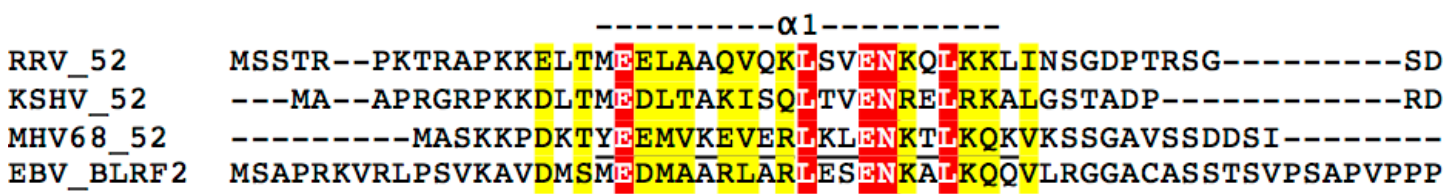

RRV_52

KSHV̄_ 52

MHV6 8

EBV_BLRF 2

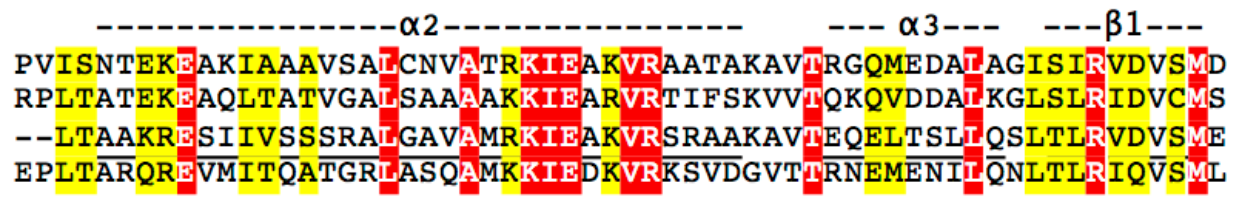

C-term RS

RRV_52

KSHV

MHV6 8 _ 52

EBV_BLRF 2

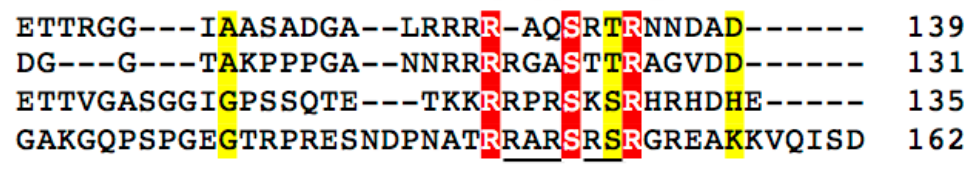


energy transfer (FRET) experiments demonstrated a self-association of ORF52. Structurally, the ORF52 monomer consists of three $\alpha$-helices followed by a $\beta$-strand near the C-terminus; however, the monomer of ORF52 is unlikely to be stable and instead exists in a dimeric or tetrameric state. The dimer exhibits 2-fold symmetry except for the $\alpha 1$ helix, which is located near the $\mathrm{N}$-terminal region (Figure 1-9). The authors suggest that this $\alpha 1$ helix mediates interactions between ORF52 and other proteins in the tegument and/or capsid. They also suggest that the asymmetrical tetramer, when not involved in virion assembly, may be a "latent" form of the protein, and the dimer may be the active form of ORF52. When the $\alpha 1$ helix moves away from the rest of the dimer, it exposes several conserved, highly hydrophobic patches on the $\alpha 1$ helix, which are optimal for mediating interaction between ORF52 and other proteins in the tegument and/or capsid of MHV-68 (24) (Figure 1-10). While there are varying levels of sequence identity and similarity among the gammaherpesvirus ORF52 proteins, data show that the $\alpha-1$ helix, as well as residue $\operatorname{Arg}^{95}$ are strictly conserved. To investigate whether these two areas may be responsible for the function of ORF52, investigators created expression plasmids deleting the first 33 amino acids of ORF52 $(\Delta 33)$ or mutating $\operatorname{Arg}^{95}$ to an alanine $(R 95 A)$. They then assessed the ability of ORF52 to self-associate and the ability of wild type or mutant ORF52 plasmid to rescue an ORF52-null mutant. Interestingly, the mutant plasmids, $\Delta 33$ or R95A, had no effect on the ability of ORF52 to selfassociate; however, both mutant plasmids were unable to rescue the ORF52-null virus, suggesting ORF52 requires both domains, to some extent, in order to 
Figure 1-9. Structure of ORF52 dimer. (A) Pymol software generated image of the ORF52 dimer. One molecule, molecule A is shown in gold, and the second, molecule $B$, is in cyan. (B) ORF52 dimer after $90^{\circ}$ rotation. (C) A model for the ORF52 dimer with interaction mediated through the $\alpha 2$ helices. (D) Conserved molecular surface features of the dimer model with residues conserved among the herpesviruses highlighted in blue and labeled. (See text for further description.) Image from (24) and used with permission. 
A

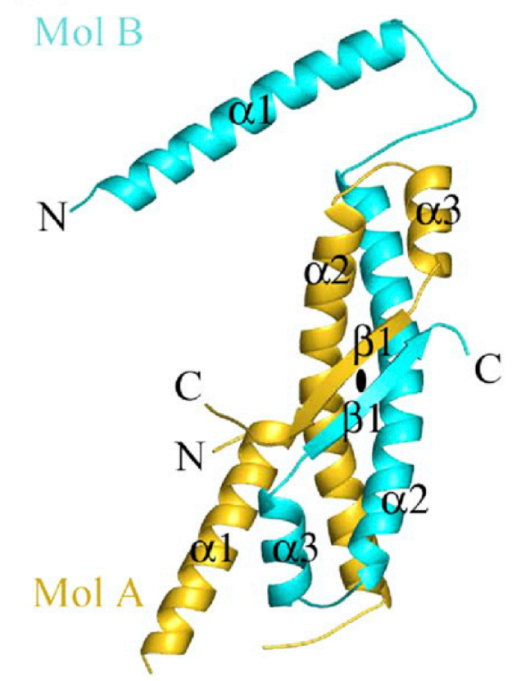

C

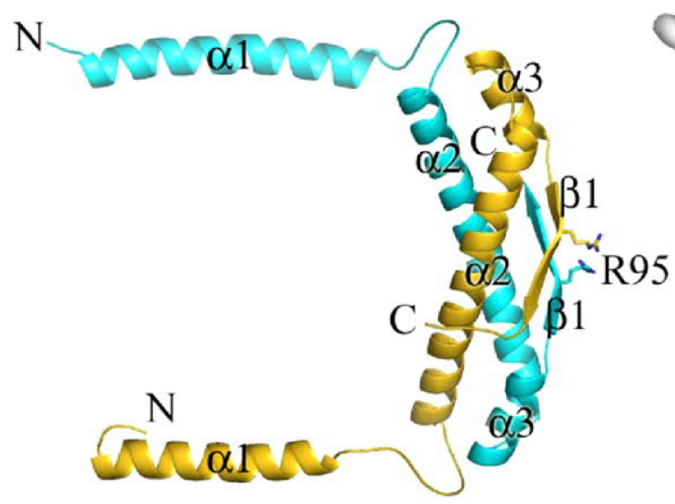

B

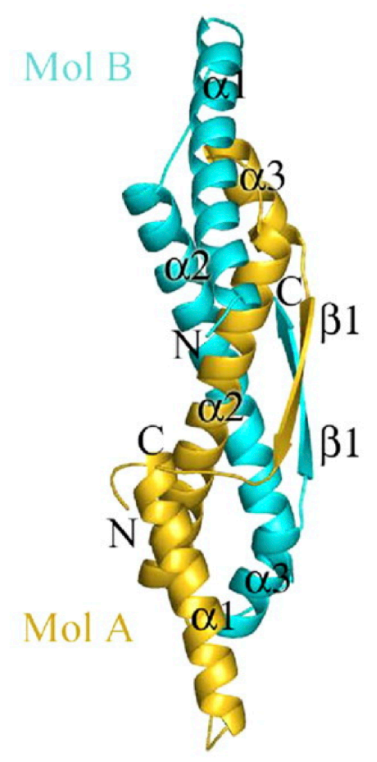

D

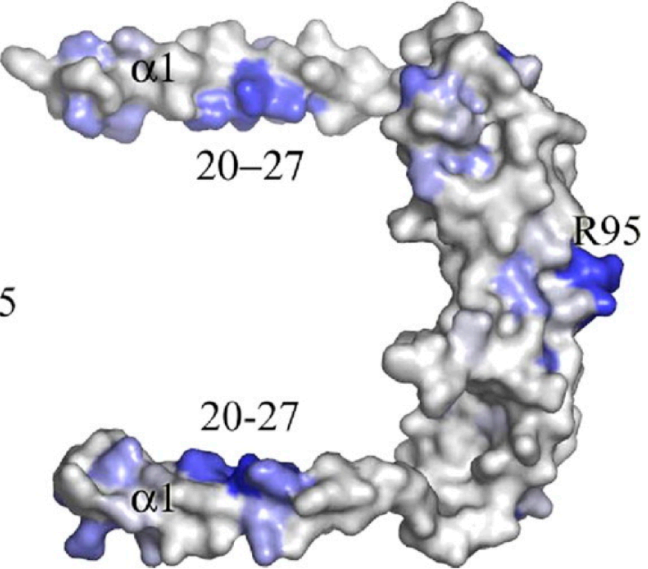


Figure 1-10. Asymmetric tetramer of ORF52. (A) Pymol software generated image of the ORF52 tetramer. The figure shows one dimer in gold and blue and another in green and magenta. (B) ORF52 tetramer after $180^{\circ}$ rotation from A. The black oval at the center of the structure is the conserved arginine 95 residue in the hydrophobic core. (See text for further description.) Image from (24) and used with permission. 
A
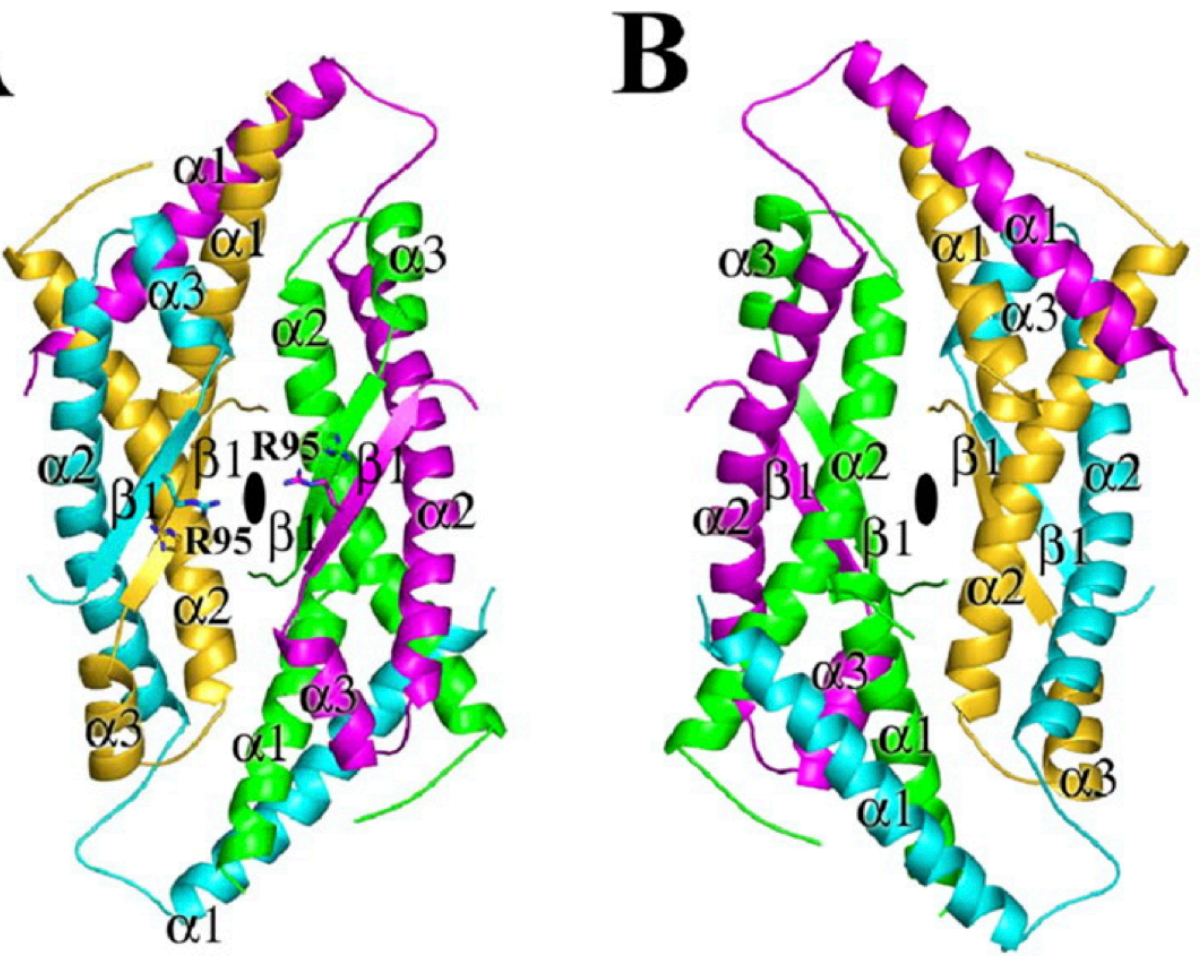
function (24).

More recent investigations in 2012 by Wang, et al. have gone on to perform a structure and function analysis, to determine the specific role of the individual MHV-68 ORF52 domains on the function of the protein (330). The authors first utilized immunoelectron microscopy (ImmunoEM) to reveal ORF52 in both mature, extracellular virions, as well as immature subviral particles within the cytoplasm. While previous research showed the $\alpha-1$ helix and C-terminal R95 to be essential to the function of ORF52 (27), here the authors demonstrate that ORF52 mutants have a dominant negative effect. 293T cells transfected with wt BAC and either a N33-del-ORF52, which lacks amino acids 1-33, corresponding to the $\alpha-1$ helix, or the R95A mutant, show a reduction of $17 \%$ and $33 \%$ respectively measured as released virus by GPCR. Transfecting these same ORF52 mutant constructs into Flp-In-293-FLAG-ORF52, which constitutively express a wt ORF52, confirmed the dominant negative effect. The researchers cotransfected 52S (Stop) BAC with wt MHV-68 ORF52 or the homologs in KSHV and EBV (ORF52 and BLRF2) and determined viral genome copies by qPCR of viral DNA in the supernatant. Both KSHV ORF52 and BLRF2 were able to rescue the defect in the 52S BAC as well as co-immunoprecipitate with wt MHV-68 FLAG-ORF52 (330).

ORF52 contains five strictly conserved amino acids $\mathrm{Leu}^{20}, \mathrm{Glu}^{23}, \mathrm{Asn}^{24}$, $\mathrm{Leu}^{27}$, and $\mathrm{Arg}^{95}$. Of these, all except $\mathrm{Arg}^{95}$ are located within the $\alpha-1$ helix and investigators reasoned that these four amino acids might be important for the function of ORF52. They made individual point mutants at each of these strictly 
conserved amino acids and co-transfected the mutant ORF52 constructs along with 52S BAC. Using qPCR they measured the ability of the ORF52 mutants to complement the defect in the $52 \mathrm{~S} B A C$ by quantifying viral DNA released into the supernatant. None of the mutants was able to complement the lack of ORF52. By performing IF on Vero cells transfected with these point mutant constructs, they found that each point mutant changed the normal localization pattern of ORF52 from its punctate cytoplasmic pattern to diffuse cytoplasmic, tight peri-nuclear, nuclear, or some combination thereof. These results suggest that the $\alpha-1$ helix is responsible for the punctate cytoplasmic localization pattern in wt ORF52 (330).

Structural data of MHV-68 indicated that ORF52 forms homodimers and that the dimeric form of ORF52 is the functional form (24). The authors suggested that the $\alpha-2$ helix domain might be responsible for dimerization (24). Additionally, the investigators performed co-IPs with FLAG-ORF52 and either wt HA-ORF52 or HA-Mdel-ORF52, which has amino acids 48-69 deleted in the a-2 helix. Western blots clearly show that FLAG-ORF52 pulls down wt-HA-ORF52 and that interaction is lost when a portion of the $\alpha-2$ helix is missing in the HAMdel-ORF52, while having no affect on ORF52 localization or staining pattern when examined by IF. Finally, the authors tested wt ORF52 for interaction with numerous other structural proteins and found it to interact with the tegument protein ORF42. By testing this interaction between wt ORF42 and different ORF52 mutant constructs, they found that the R95A ORF52 mutant resulted in a loss of interaction with ORF42. The role of ORF42 is currently unknown. As a final note, in a paper by Duarte, et al., the authors reported in an interaction 
table, without showing the data, that MHV-68 ORF52 interacts with ORF45 (EBV BKRF4) (82). This interaction between ORF52 and ORF45 is of particular interest to our work on the virion protein-protein interactome.

\section{KSHV ORF52}

KSHV ORF52 is 131 amino acids long and is $47 \%$ identical and $67 \%$ similar to RRV ORF52 and 34\% identical and $64 \%$ similar to MHV-68 ORF52 (NCBI protein BLAST). A study by Rozen, et al. in 2008 sought to investigate the protein-protein interactions occurring within the KSHV virion by both yeast twohybrid and subsequent confirmation by coimmunoprecipation. They detected thirty-seven protein-protein interactions during this investigation. KSHV ORF52 interacted with the capsid protein TRI-2 (ORF26), two tegument proteins (ORF45 and ORF75), and two envelope glycoproteins ( $\mathrm{gM}$ and $\mathrm{gN}$ ) in coimmunoprecipitation experiments, as well as by the yeast two-hybrid method when ORF52 was used as bait (267). Additionally, ORF52 interacted with a third tegument protein, ORF64N, or large tegument protein, (with the ' $N$ ' labeling for a truncated form of ORF64), by the yeast two-hybrid method, but not by co-IP. KSHV ORF52, when expressed in isolation, has a diffuse cytoplasmic staining pattern ((274) and our own unpublished observations).

\section{EBV BLRF2 (ORF52 homolog)}

BLRF2 is the EBV homolog of ORF52. It is 162 amino acids long (142) and has $38 \%$ identity and $55 \%$ similarity to RRV ORF52 (NCBI protein BLAST). Previous 
reports showed that BLRF2 interacts with another tegument protein, BNRF1 (the RRV-, KSHV-, and MHV-68- ORF75 homolog) (38). An additional study, using a yeast-two hybrid approach, identified several cellular proteins that interacted with BLRF2 including Serine/Arginine-rich protein kinase 2 (SRPK2) (268). SRPK2 is part of a larger family of serine/arginine kinases that phosphorylate serine residues in arginine-serine (RS) motifs and play critical roles in pre-mRNA splicing, acting as non-small nuclear ribonuclearprotein (snRNP) factors ((329) and reviewed in $(98,109))$. Additionally, SRPK2 induces cell cycle progression by increasing cyclin A1 expression in hematopoietic cells (139) and apoptosis in neurons by increasing cyclin D1 (138).

A recent study of BLRF2 by Duarte, et al., further confirmed potential interactions by co-IP between cellular proteins and BLRF2 and found that BLRF2 does indeed interact with SRPK2 through the C-terminus of BLRF2 (82). BLRF2 has two arginine-serine (RS) repeats in the C-terminal domain that were found to be substrates for SRPK2 phosphorylation. Taking advantage of an MHV-68 ORF52 null BAC, the investigators found that a BLRF2 construct with the RS motifs changed to ARA was unable to rescue viral production where a wt BLRF2 could. Interestingly, in contrast to its homologs in RRV and MHV-68, EBV BLRF2 has a nuclear staining pattern, that is relocalized to the cytoplasm during the course of EBV infection (82).

\section{Gammaherpesvirus ORF45}

The gammaherpesvirus protein ORF45 is a tegument protein found within the 
virions of RRV (239), MHV-68 (28), $\operatorname{KSHV~}(23,358)$, and EBV (142). See Figure 1-11 for alignment.

\section{RRV ORF45}

RRV ORF45 is 353 amino acids long, is highly phosphorylated, has a nuclear staining pattern, and shares relatively high sequence identity to other gammaspecific ORF45 tegument proteins (see homologs below for specific percentage) predominantly in the C-terminal region. ORF45 interacts selectively with activated pERK2 to promote nuclear accumulation and protection of $\mathrm{pERK}$ from phosphatases, promoting lytic viral gene expression and preserving persistent and robust activation of both nuclear and cytoplasmic ERK targets (340). Knockdown of RRV ORF45 leads to a decrease in ORF52 protein in the cell and a decrease of infectious virus produced (Anderson and Kedes, Appendix 5, Figure 5-1).

\section{MHV-68 ORF45}

In MHV-68, ORF45 is a highly phosphorylated immediate-early tegument protein of 206 amino acids with a primarily nuclear staining pattern of expression (27, 141). MHV-68 is $53 \%$ identical and $57 \%$ similar to RRV ORF45 (NCBI protein BLAST). MHV-68 ORF45 shows less association with the viral capsid than ORF52 and researchers consider it an outer tegument protein (27). The role of ORF45 is not entirely clear at this point, however, it appears to be important for viral gene expression. Transfection of a 45STOP (45S) BAC resulted in a defect 
Figure 1-11. Alignment of ORF45 in RRV, KSHV, MHV-68, and EBV. Strictly conserved residues are highlighted in red and conservatively conserved residues in yellow. Alignment done using CLUSTAL O (1.2.1) software (http://www.ebi.ac.uk/Tools/msa/clustalo/). 


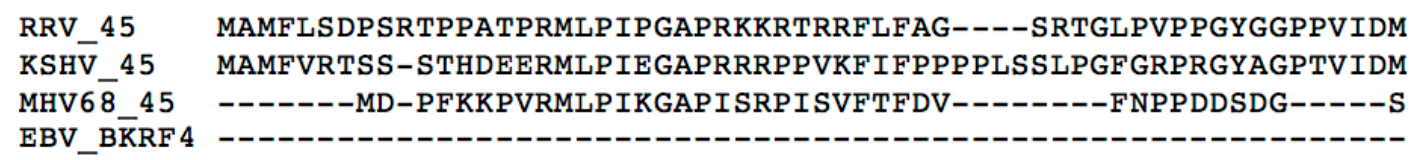

RRV_45 TAPEDVFDQDSPPTTPKTPDET---DSHSENSDYS-----------DVDEEDEPPVSSP KSHV 45 SAPDDVFAEDTPSPPATPLDLQISPDQSSGESEYDEDEEDEDEEENDDVQEEDEPEGY-MHV6 $\overline{8}$ 45 STPDSVFEAE---

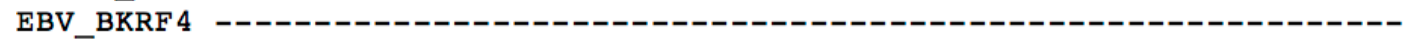

RRV_45 PRIDPHARDGESFNQSGRLPTVITSTGATTPPSAPAPL-TAFGGPRPVAVVTGQHRAPQS KSHV _45 -------PADFFQPLSHL------------RPRPLARRAHTPKPVAVVAGRVRSSTD MHV6 $\overline{8}-45$ EBV_BK

RRV_45 KSHV 45

SESDSED-DFFI------DDYEDTD-----------ESGGEADGF SPRASPAWSGDIS MHV6 $\overline{8}-45$ TAESEASMGWVSQDDGFSPAGLSPSDDEGVAILEPMAAYTGTGAYGLSPASRNSVPGTQS EBV_BKRF 4 ------MAMFLKSRGVRSCR-----DRRLL-SDEEEETSQSSSYTL------------

RRV 45 KSHV̄ 45 MHV $6 \overline{8} \quad 45$ ---RSPAE-GGWSSDEEEEPVVAGSNAGQETIIISDDDEVDDRGS------------EBV_BKRF 4

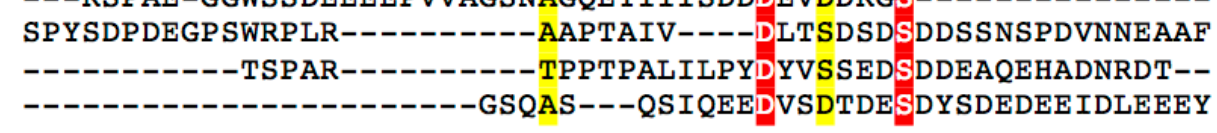

RRV_45 ---------VETW----------DESDADEGTGATDVIDLCSSSDSDDDADHVT KSHV _45 TD-------ARHFSHQPPSS--------EEDGEDQGEVLSQRIGLMDVGQKRKRQSTAS MHV6 $\overline{8} 45$--------NDQVSESSTSE--------DSDSESESSCDDTQQTFVD------DESTSS EBV_BKRF 4 PSDEDPSEGSDSDPSWHPSDSDESDYSESDEDEATPGSQASRSSRVSPS-------TQQS

RRV_45 SGG-VRAACKRRASRR-D--------------CNGDDD--VIY-VGTT------QGPKR KSHV _45 SGSEDVVRCQRQPNLSRKAVASVIIISSGSDTDEEPSSAVSVIVSPSSTKGHLPTQSPST MHV6 8 _ 45 SGEEDRDD----PFESND------QGAQGGSTSES-EDDLPAILRA-----ARETQSDSEBV_BKRF 4 SGLTPTPSF-SRPRTRAPPRPPAPAPVRG-RASAPPRPPAPVQQSTK-------DKGPHR

RRV 45 RMTST---TGAGATSNPEGPGVSGRQTMAATPPVCGNDNȲPWTPLD 353

KSHV 45 SAH-S---ISSGSTTTAGSRCSDPTRILASTPPLCGNGAYNWPWLD 406

MHV6 8 _45 -SS-D---SSGNSHKKRRVQEEESSRILKTPAPISGNGKYNWPWLD 206

EBV_BKRF 4 PTRPVLRGPAPRRPPPPSSPNTYNKHMMETTPPIKGNNNYNWPWI- 217 
in viral replication, as well as lack of expression of the late lytic proteins ORF25 and ORF65. Researchers mapped the role in viral replication to the $23 \mathrm{C}$ terminal amino acids and rescued the defect by providing MHV-68 ORF45 in trans (141).

\section{KSHV ORF45}

KSHV ORF45 is an immediate-early gene (359) that encodes a phosphorylated tegument protein of 407 amino acids present in the virion particle $(358,363)$. KSHV ORF45 is $49 \%$ identical and $58 \%$ similar in sequence to RRV ORF45 (NCBI protein BLAST). In contrast to its homolog in MHV-68 and RRV, KSHV ORF45 has a predominantly cytoplasmic localization, although it has both a nuclear export and import signal and it does shuttle back and forth $(177,274)$. Interestingly, this localization appears to have a role in lytic replication, as recombinant viruses with a mutation that causes cytoplasmic only localization produced 5- to 10- fold fewer viruses than control, whereas recombinant virions restricted to the nucleus produced virions similar to control (177). There also appears to be possible interactions between ORF45 and other viral proteins when examined by yeast-two hybrid, co-IP, or both. These include the capsid proteins ORF26, ORF62, and ORF65 as well as several tegument proteins, ORFs $11,21,27,33,63,64 \mathrm{M}$ ('M' is one fragment of the whole ORF64 protein) and 75 (note that when ORF45 is used as bait, ORF52 does not interact by yeast two-hybrid or co-IP) and the glycoproteins gM and gH (267). ORF45 also plays a role in viral egress. ORF45 is responsible for docking viral capsid-tegument 
particles onto the kinesin-2 motor protein, KIF3A subunit for movement along microtubules toward the cell periphery (277).

Extensive study of KSHV ORF45 has found it to have several important roles within the virus life cycle. While there was no significant difference in viral gene expression or lytic DNA replication, cells transfected with an ORF45-Stop BAC (45S BAC) released 10-fold fewer virions than the wt BAC. Additionally, these virions were less infective than those produced in wt conditions, suggesting that KSHV ORF45 may play roles both in early and late stages of infection (361). The cellular protein seven in absenthia homolog (SIAH), which is in the ubiquitin E3 ligase family, also interacts with ORF45 and stimulates degradation of ORF45 through the proteasome (1). As ORF45 is important in viral infection, targeting degradation of the protein may dampen the ability of the virus to establish a productive infection.

ORF45 plays additional roles in the cellular immune response to viral infection. ORF45 associates with the inhibitory domain of cellular interferonregulatory factor 7 (IRF-7), thereby inhibiting its phosphorylation by inhibitor-KB kinase $\varepsilon$ (IKK $\varepsilon$ ) or TANK binding kinase 1 (TBK1). By acting as an alternative substrate, ORF45 inhibits activation of IRF-7, preventing its translocation to the nucleus. The inability of IRF-7 to translocate into the nucleus prevents activation of interferons alpha and beta, which are key factors in the immune system response to viral infection $(178,276,360,362)$.

ORF45 also interacts with p90 ribosomal S6 kinases RSK1 and RSK2, stimulating their kinase abilities (169). The activation of RSKs by ORF45 occurs 
as ORF45 interacts with —and forms a complex with - RSK and ERK. These complexes protect the activated phosphorylated forms of ERK and RSK (pERK and pRSK) from dephosphorylation, leading to an increase in persistent activation in KSHV (170). This persistent activation of RSK by ORF45 also causes an increase in phosphorylation and activation of eukaryotic translation factor 4B (elF4B), which increases viral replication and progeny virion production (168).

\section{EBV BKRF4 (ORF45 homolog)}

EBV BKRF4 is 217 amino acids long and shares $46 \%$ identity and $62 \%$ similarity to RRV ORF45 (NCBI protein BLAST). No additional information is known at this time with respect to BKRF4 function.

\section{Gammaherpesvirus ORF75}

Gammaherpesvirus ORF75 is a tegument protein found within the virions of RRV (239), MHV-68 (28), KSHV (23, 102, 358), EBV (142), and herpesvirus saimiri (HVS) (159). ORF75 is a member of the viral phosphoribosylformylglycineamide amidotransferase (FGARAT) family of proteins. Cellular FGARAT proteins are involved in purine biosynthesis (247); however, in herpesviruses the FGARAT family of proteins reportedly plays a role in counteracting a group of cellular restriction factors called nuclear domain 10 (ND10) that inhibit herpesvirus lytic DNA replication. ND10 components include

promyeloctic leukemia protein (PML), speckled protein 100 (Sp100), death 
domain-associated protein (Daxx), and ATP-dependent helicase (ATRX) ((306) and reviewed in (262)).

\section{MHV-68 ORF75}

In MHV-68 ORF75c is 1310 amino acids with $27 \%$ identity and $43 \%$ similarity to RRV ORF75 (NCBI protein BLAST). ORF75c induces proteasomal degradation of PML complexes leading to a dispersal of ND10 components and a reduction in SUMOylation, Daxx and Sp100 levels (108, 180). Researchers found that ORF75c interacts weakly with PML and might mediate subsequent proteasomal degradation by direct ubiquitination of PML by ORF75 (284).

\section{KSHV ORF75}

Made up of 1296 amino acids, KSHV ORF75 shares 44\% identity and 59\% similarity with RRV ORF75 (NCBI protein BLAST). KSHV ORF75 is essential for viral replication and initiation of viral immediate-early gene expression (102). When expressed in isolation following transfection, KSHV ORF75 displays both nuclear and cytoplasmic staining pattern (274). KSHV infection leads to a reduction of ATRX and a dispersion of Daxx that researchers also note when the cell expresses ORF75 in isolation. The ND10 components PML and Sp100 (102) restrict KSHV. Additionally, when used as bait in yeast-two-hybrid or co-IP experiments, KSHV ORF75 interacted with ORF62 (TRI-1), ORF21, ORF64M, $\mathrm{gM}$, and $\mathrm{gN}$. As mentioned in their respective sections, ORF75 interacted with ORF52 and ORF45 only when ORF75 was the prey (267). 


\section{EBV BNRF1 (ORF75 homolog)}

EBV BNRF1, the ORF75 homolog, is 1318 amino acids with $31 \%$ identity and 44\% similarity to RRV ORF75. BNRF1 interacts with the ND10 component Daxx, enabling viral replication by releasing ATRX from ND10 (317).

\section{Gammaherpesvirus ORF27}

RRV ORF27 is identified as one of the five gammaherpesvirus specific tegument proteins (239). Investigations into the specific function of ORF27 have only begun in MHV-68, but suggest that ORF27 encodes a type 2 transmembrane glycoprotein (gp48) found on the surface of infected cells and within the viral particle that, when disrupted, impair cell-to-cell spread of virus $(196,197)$. KSHV ORF27 is listed as an "uncategorized" protein (358) with a cytoplasmic staining pattern (274), and EBV BDLF2 is listed as a "probable tegument protein" (142).

\section{Gammaherpesvirus ORF49}

KSHV ORF49 displays a nuclear localization pattern (274). There is currently no published information as to the function of ORF49.

This thesis focuses on the function of RRV ORF52, but at different points also discusses the $\mathrm{Y}$-specific tegument proteins, ORF45 and ORF75, in addition to other proteins conserved across the Herpesviridae family, including ORF33 and ORF64. 
CHAPTER 2. MATURATION AND VESICLE-MEDIATED EGRESS OF PRIMATE GAMMAHERPESVIRUS RRV REQUIRE INNER TEGUMENT PROTEIN ORF52 


\section{ABSTRACT}

The tegument layer of herpesviruses comprises a collection of proteins that is unique to each viral species. In Rhesus monkey rhadinovirus (RRV), a close relative of the human oncogenic pathogen, Kaposi's sarcoma-associated herpesvirus, ORF52 is a highly abundant tegument protein tightly associated with the capsid. We now report that ORF52 knockdown during RRV infection of Rhesus fibroblasts led to a greater than 300 -fold reduction in viral titer by $48 \mathrm{~h}$, but had little effect on the number of released particles, only modest reductions in the levels of intracellular viral genomic DNA and no appreciable change in viral DNA packaging into capsids. These data suggested that the lack of ORF52 resulted in the production and release of defective particles. In support of this interpretation, transmission electron microscopy (TEM) revealed that without ORF52, capsid-like particles accumulated in the cytoplasm and were unable to enter egress vesicles where final tegumentation and envelopment normally occurs. TEM also demonstrated defective particles in the media that closely resembled the accumulating intracellular particles, having neither a full tegument nor an envelope. The disruption in tegument formation from ORF52 suppression, thereby, prevented the incorporation of ORF45, restricting its subcellular localization to the nucleus and appearing, by confocal microscopy, to inhibit particle transport toward the periphery. Ectopic expression of siRNA-resistant ORF52 was able to partially rescue all of these phenotypic changes. In sum, our results indicate that efficient egress of maturing virions and, in agreement with 
studies on MHV-68, complete tegumentation and secondary envelopment are dependent on intact ORF52.

\section{INTRODUCTION}

Rhesus monkey rhadinovirus (RRV), a gamma-2 herpesvirus, is a close homolog of Kaposi's sarcoma associated herpesvirus (KSHV) or HHV8, the causative agent of three human tumors: Kaposi Sarcoma, primary effusion lymphoma and multicentric Castleman's disease $(46,47,52)$. Like all herpesviruses, KSHV and RRV have both latent and lytic phases of infection (reviewed in $(29,117,211)$ ). KSHV adopts a primarily latent infection and, even with reactivation by phorbol esters or histone deacetylace (HDAC) inhibitors, titers in culture are low $(94,259)$. In contrast, RRV efficiently infects cultured primary or immortalized Rhesus monkey fibroblasts (RhF), enters the lytic phase, and replicates to relatively high viral titer (69). These qualities, along with the high levels of conservation in their genomic sequence and organization $(12,281)$, make RRV a useful model to study the structure and lytic (productive) replication of KSHV and other gammaherpesviruses, as well as the role(s) of individual lytic viral genes in these processes.

RRV also shares with all members of the Herpesviridae family the same overall structural architecture: a linear double stranded DNA genome surrounded by an icosahedral capsid, then a proteinaceous layer or tegument, comprised of a limited subset of viral and cellular proteins, and finally an outermost layer derived from the host cell envelope studded with viral glycoproteins (reviewed in 
(211)). During host cell entry, the herpesvirus envelope fuses with either the plasma membrane or an endocytic vesicle membrane, releasing the majority of tegument proteins $((113,195)$ and reviewed in $(50))$. Investigators have suggested that these tegument proteins likely play important roles in preparing the host cell for viral replication $(23,124,156,157,200,279,302)$. Though the process of tegumentation remains poorly defined, previous studies have found both cellular and viral proteins within this layer $(142,184,239,298,325,358)$. This gives rise to a proteomic tegument profile that is distinct for each different viral species and possibly even among the same species grown within different cell types or conditions (341).

Until recently, structural information for the tegument was limited to images from electron microscopy (EM) that suggested that this layer was comprised of an amorphous collection of proteins; however, more recent studies with human cytomegalovirus (HCMV) (55), HSV-1 (36, 356), MHV-68 (62), RRV (349), and KSHV (267) indicate the presence of ordered tegument structures (356). Investigators have further divided the tegument into inner and outer layers, which are evident by cryo-electron tomography of MHV-68 (62), with inner tegument proteins more tightly associated with the capsid and more resistant to Triton X-100 detergent treatment $(239,336,356)$. The majority of functional data regarding the herpesvirus tegument comes primarily from research on alpha and betaherpesviruses, which has shown that tegument proteins function in crucial roles in viral replication, including transcytosis of the herpesvirus capsid toward the nucleus during initial infection and egress from the nucleus toward the 
periphery during lytic replication (27, 113, 187, 295, 350). Additional functions of tegument proteins include modulation of the host cell environment during the immediate-early phase of infection (294), including shut off of host gene expression $(289,301,302)$, antagonism of innate antiviral host response $(102$, $147,178,180,276,362)$, and assembly and egress of herpesvirus virions ((27, $57,120,277)$ and reviewed in (213)).

Previous biochemical and mass spectrometry analyses of Rhesus Monkey Rhadinovirus (RRV) from our laboratory indicate the presence of at least 33 virally encoded proteins comprising the viral particles (239). Among these proteins in RRV, we identified ORF52 as a gammaherpesvirus specific, highly abundant tegument protein that tightly associates with the capsid (239), but with a function that remains uncharacterized for this primate gammaherpesvirus. ORF52 encoded by RRV is a late gene (73), encoding a protein of 139 amino acids with a molecular weight of $15 \mathrm{kDa}$. Homologs are present within other gammaherpesvirus virions and share varying degrees of identity/similarity, including MHV-68 (41\%/63\%) $(27,28), \mathrm{KSHV}(47 \% / 67 \%)(23,358)$, and EBV (38\%/55\%) (142). MHV-68 ORF52 plays a key role in tegumentation and secondary envelopment of viral particles $(27,330)$.

In the present study, we have examined the function of ORF52 during lytic infection with wild type RRV in culture using siRNA knockdown and rescue approaches. We assessed the role of ORF52 on various steps during the viral life cycle, including DNA replication and packaging, capsid production and assembly, nuclear egress, tegumentation and envelopment, and finally egress with the 
production and release of infectious virions. Our results argue that, much like its murine homolog from MHV-68, RRV ORF52 is necessary for late stages in the viral life cycle and that when ORF52 is absent or limited, particles fail to undergo tegumentation and secondary envelopment. We additionally found that in the absence (or severe reduction) of ORF52, another tegument protein, ORF45, remained restricted to the nucleus. Furthermore, and in contrast to infections with MHV-68 containing a stop codon in its ORF52 homolog, RRV ORF52 knockdown still resulted in the release of subviral particles that lacked tegument and envelope and were also unable to remain cell-surface associated, unlike mature and fully infectious virions.

\section{MATERIALS AND METHODS}

\section{Cell Culture}

Telomerase-immortalized rhesus monkey fibroblasts (RhF) were grown in complete media (Dulbecco's modified Eagle's medium [Gibco] supplemented with $1 \mathrm{nM}$ puromycin, $1 \mathrm{mM}$ sodium pyruvate, and $10 \%$ fetal bovine serum [Gibco]), as described previously (239). HEK293 cells were grown in complete media (Dulbecco's modified Eagle's medium [Gibco] supplemented with 10\% fetal bovine serum.

\section{RRV Stocks}

RhFs were grown to confluency, approximately $2 \times 10^{7}$ cells in a T182 flask, and infected with RRV strain $\mathrm{H} 26-95$ at a multiplicity of infection (MOI) of $0.05 \mathrm{in} 5 \mathrm{ml}$ 
complete media for 1 hour. Cells were then supplemented with an additional $100 \mathrm{ml}$ of complete media per flask. Media was collected 5-7 days post-infection (p.i.) and cleared of cellular debris by low-speed centrifugation at $350 \times \mathrm{g}$. Cleared media containing virus were passed through a $0.45 \mu \mathrm{m}$ pore size filter. Virus was concentrated by centrifugation for 3 hour at $12,855 \times \mathrm{g}$ in a Sorvall SL250T rotor. Resulting viral pellets were resuspended in $1.0 \mathrm{ml}$ TNE $(20 \mathrm{mM}$ Tris $[\mathrm{pH} 7.5], 100 \mathrm{mM} \mathrm{NaCl}, 1 \mathrm{mM}$ EDTA), titer was determined, and $20 \mu \mathrm{L}$ aliquots were stored at $-80^{\circ} \mathrm{C}$.

\section{Antibodies}

Mouse monoclonal anti-RRV ORF52 and anti-RRV ORF65 (SCIP) were generated in the Lymphocyte Culture Center at the University of Virginia. Following PCR amplification and cloning of RRV orf52 and orf65 from RRVderived DNA, full-length ORF52 and ORF65 (SCIP) GST-fusion proteins were made in E. coli. Following purification and cleavage of GST, the proteins were used by the Lymphocyte Culture Center to produce mouse monoclonals to ORF52 and ORF65/SCIP. Rabbit polyclonal anti-RRV ORF45 was generated from Open Biosystems, Inc. (341). Anti-RRV major capsid protein (MCP) was kindly provided by Scott Wong at Oregon Health and Science University. Rabbit polyclonal anti-actin (sc-1616-R) was purchased from Santa Cruz Biotechnology.

Anti-Infrared Dye 800 anti-mouse and Infrared Dye 800 anti-rabbit were purchased from LiCor Biosciences and Rockland Immunochemicals, respectively. 
SiRNA

Silencer Select custom siRNA specific to the RRV ORF52 coding sequence 5'AACCCGTAAGATTGAAGCTAA-3' and siControl \#1 were purchased from Life Technologies.

\section{SiRNA transfection followed by RRV infection}

$20 \mathrm{nM}$ of ORF52 siRNA or Control siRNA were transfected into RhF in $10 \mathrm{~cm}^{2}$ plates using Lipofectamine RNAiMAX (Life Technologies) following the Reverse Transfection manufacturer protocol. 24 hours later, cells were infected with RRV at an $\mathrm{MOI}$ of 5 for 1 hour at $37^{\circ} \mathrm{C}$ with rocking every 15 minutes to ensure uniform distribution of virus. 1 hour later, virus was removed and cells were washed with 1X phosphate buffered saline (PBS) 3 times and then replaced with complete media. Cells were incubated for an additional 48 hour.

\section{Expression of viral lytic proteins}

\section{(i) RRV Infected RhF}

Following removal of the media, plated cells were washed 3 times in $1 \mathrm{X}$ PBS at room temperature. Cells were trypsinized off plates and pelleted. Pellets were washed once in 1X PBS prior to being resuspended in lysis buffer as described above. For each sample, approximately $45 \mu \mathrm{g}$ of total protein was loaded onto a pre-cast $12 \%$ BisTris gel (NuPage; Life Technologies). 


\section{(ii) Viral Supernatants}

$48 \mathrm{~h}$ p.i. media was collected and cell debris cleared by centrifugation at $350 \times \mathrm{g}$. To concentrate and isolate viral particles, $3 \mathrm{~mL}$ of the cleared media was layered over $750 \mu \mathrm{L}$ of a $20 \%$ sucrose cushion in TNE. Media was centrifuged at 65,204 $\mathrm{xg}$ for 30 minutes at $4^{\circ} \mathrm{C}$ in a SW-41Ti rotor. Following centrifugation, media was decanted and pellets containing viral particles were resuspended in $60 \mu \mathrm{l}$ sample buffer. Samples were boiled for 10 minutes and equal volumes were loaded onto a $12 \%$ BisTris gel. Statistical significance between conditions was determined using an unpaired Student's $t$ test. Calculations were performed using GraphPad Prism online software.

\section{Protein electrophoresis and immunoblotting}

Cells were trypsinized off of plates, pelleted, and washed once in 1X PBS. Pelleted cells were lysed for 15 minutes at $4^{\circ} \mathrm{C}$ with whole-cell lysis buffer (50 $\mathrm{mM}$ Tris [pH 7.3], $150 \mathrm{mM} \mathrm{NaCl}, 1 \%$ Igepal, $5 \mathrm{mM}$ EDTA, 10\% glycerol) supplemented with $1 \mathrm{X}$ protease inhibitor cocktail (Roche Applied Science) immediately prior to use. Lysed cells were centrifuged for 30 minutes at $4^{\circ} \mathrm{C}$ and media were removed for protein analysis.

Cell lysates and concentrated supernatants containing viral particles from each experiment were resuspended in lithium dodecyl sulfate LDS sample buffer (NuPage; Life Technologies) with NuPage sample reducing agent $(50 \mathrm{mM}$ dithiothreitol (DTT)). Following denaturation by boiling for 10 minutes, proteins were separated by sodium dodecyl sulfate polyacrylamide gel electrophoresis 
(SDS-PAGE) on 12\% Bis-Tris gels (NuPage; Life Technologies).

For immunoblot analyses, proteins separated by SDS-PAGE were transferred to nitrocellulose membranes for 60 minutes at $350 \mathrm{~mA}$ at $4^{\circ} \mathrm{C}$. The membranes were blocked in 5\% nonfat milk-TBS $(20 \mathrm{mM}$ Tris base, $150 \mathrm{mM}$ $\mathrm{NaCl}, 3 \mathrm{mM}$ Tris- $\mathrm{HCl}$ ) for 90 minutes at room temperature and then incubated with primary antibodies overnight at $4^{\circ} \mathrm{C}$. Primary antibodies were used at the following dilutions: anti-RRV ORF52 (1:1,000), anti-RRV $\operatorname{SCIP}(1: 2,500)$, antiRRV ORF45 (1:10,000), anti-RRV MCP $(1: 1,000)$, and anti-mouse actin $(1: 15,000)$. After three washes with TBS-Tween $(0.05 \%)$ at room temperature (RT), membranes were incubated with secondary antibodies for 45 minutes at RT. For quantitative immunoblotting membranes were incubated with Infrared Dye 800-conjugated anti-mouse (Rockland Immunochemicals) or Infrared Dye 800-conjugated anti-rabbit (LiCor Biosciences) diluted 1:10,000 in 5\% nonfat milk in TBS-Tween (0.05\%). Images were scanned and analyzed using an Odyssey infrared imaging system and 3.0 software (LiCor Biosciences).

\section{Quantitative PCR (qPCR)}

\section{(i) RRV Infected RhF}

Cells from a 6 well plate were washed 3 times in 1 X PBS prior to collection and after pelleting were resuspended in PK digestion buffer $(100 \mathrm{mM} \mathrm{NaCl}, 10 \mathrm{mM}$ Tris $\mathrm{Cl}$ [pH. 8], $25 \mathrm{mM}$ EDTA [pH. 8], 0.5\% SDS and $0.1 \mathrm{mg} / \mathrm{ml}$ PK (SigmaAldrich) and incubated at $55^{\circ} \mathrm{C}$ overnight. DNA was extracted as above. A standard DNA concentration curve was based on serial dilutions of an ORF45 
plasmid, pCMV-Tag2A-ORF45. Primers for human glyceraldehyde-3-

phosphate dehydrogenase

(5'GAAGATGGTGATGGGATTTCCA-3')
(GAPDH),

and
GAPDH-F

GAPDH-R

(5'GATTCCACCCATGGCAAATT3'), were used to normalize the samples. Biological triplicates were performed for each condition and time point and each triplicate was analyzed by PCR in triplicate. Quantitative data are presented as means and error bars for only the positive component of the SD.

\section{(ii) Viral supernatants}

Pre-cleared media was collected under each condition at various time points post-infection (p.i.) and particles pelleted by centrifugation at $65,204 \times \mathrm{g}$ for 30 minutes at $4{ }^{\circ} \mathrm{C}$ in a SW-41Ti rotor through $750 \mu \mathrm{L}$ of a $20 \%$ sucrose cushion. The pellet containing viral particles was resuspended in DNase buffer (100 mM Tris$\mathrm{Cl} ; 25 \mathrm{mM} \mathrm{MgCl} ; 1 \mathrm{mM} \mathrm{CaCl}_{2}$ ) in PBS overnight at $4^{\circ} \mathrm{C}$. Viral particles were then dissociated by bath sonication in an ice bath slurry 5 times for 3 seconds each, and samples were treated with $5 \cup$ RNAase free DNase I (Stratagene) for 30 minutes at $37^{\circ} \mathrm{C}$ to leave only encapsidated DNA. DNase I was inactivated with $50 \mathrm{mM}$ EDTA and an additional 10 minutes of incubation at $65^{\circ} \mathrm{C}$ to inactivate the

DNase. Samples were then treated with $0.1 \mathrm{mg} / \mathrm{mL}$ proteinase K (PK) (SigmaAldrich) at $55^{\circ} \mathrm{C}$ overnight. DNA was extracted with phenol-chloroform and precipitated with ethanol along with $1 \mu \mathrm{g}$ of glycogen carrier (Glycoblue; Ambion). RRV genomic copy numbers were assayed in triplicate by real-time PCR (SYBR green PCR Master Mix; Applied Biosystems), using primers specific to the 
ORF45 coding sequence (ORF45F, 5'TGATTCGTCCCATGTCTCAA3'; ORF45R, 5'CCTGTTGTTGCTGGATCAAA3') and amplified and detected with an $\mathrm{ABI}$ Prism $7900 \mathrm{HT}$ detection system instrument at the University of Virginia Biomolecular Research Center. Quantification was based on serial dilution of a plasmid bearing the ORF45 coding sequence, pCMV-Tag2A-ORF45.

\section{Plaque Assay}

Determination of viral titers was performed essentially as described previously (71). In brief, RhF were plated in 12-well plates. 48 hours later, confluent monolayers were infected with 5-fold serial dilutions of pre-cleared media containing an unknown concentration of virus. Each dilution was done in triplicate. Plates were incubated for 1 hour at $37^{\circ} \mathrm{C}$ with rocking every 15 minutes to ensure uniform distribution of the virus. After 1 hour, overlay media containing $0.6 \%$ methyl-cellulose was added. Plates were incubated for 5 days at $37^{\circ} \mathrm{C}$. Overlay media was removed and cells were stained with crystal violet for 10 minutes at room temperature. Plaques were counted using an inverted microscope (Nikon Eclipse TE-2000-E) at X 10 magnification. Absolute titers were determined based on the dilution used and the number of plaques counted. Data are presented as means and SD. Statistical significance between conditions was determined using an unpaired Student's $t$ test.

\section{IF Assay}

20,000 cells/well were reverse transfected with RNAiMax (Life Technologies) 
with either ORF52 siRNA or control siRNA and plated onto 48 well plates containing a Cell-Tak (BD Biosciences) coated $8 \mathrm{~mm}$ round coverslip (Electron Microscopy Sciences) and incubated for 24 hours. Cells were then infected with RRV at a MOI of 5 or mock infected.

$48 \mathrm{~h}$ p.i., cells were fixed with $4 \%$ formaldehyde in PHEM buffer $(60 \mathrm{mM}$ PIPES, $25 \mathrm{mM}$ HEPES, $10 \mathrm{mM}$ EGTA, $2 \mathrm{mM} \mathrm{MgCl}$, pH 6.9) solution for 15 minutes at room temp. Cells were washed $3 x$ with PHEM buffer and then permeabilized in $0.25 \%$ triton in PHEM buffer for 10 minutes and washed $3 x$ with PHEM buffer. The samples were blocked overnight at $4 \mathrm{C}$ in $10 \%$ normal goat serum (in PHEM).

Samples were stained at room temperature with antibodies diluted in $5 \%$ normal goat serum (in PHEM). Primary antibodies were incubated for 1 hour, and secondary antibodies were incubated for 30 minutes. The staining was sequential (i.e. primary then corresponding secondary followed by the next primary and its corresponding secondary and so forth) with 3x washes of PHEM buffer between each antibody. Primary antibodies to ORF52 (1:500), ORF45 (1:250), and SCIP (1:250), conjugated to 488 using the Mix-n-Stain CF488A kit (Biotium) were used. Secondary antibodies Alexa Fluor 647 goat anti-mouse (ORF52) and Alexa Fluor 555 goat anti-rabbit (ORF45) (both 1:500) (Life Technologies), respectively were used. After the last secondary antibody, cells were counterstained with DAPI (4,6-diamidino-2-phenylindole; Sigma) $(1.0 \mu \mathrm{g} / \mathrm{mL}$ in double distilled water) for 5 minutes and washed $1 \mathrm{x}$ with double distilled water. Cover slips were mounted onto microscope slides (Fisher) with Fluro-Gel 
(Electron Microscopy Sciences) and imaged using the Zeiss 710 confocal microscope.

\section{Transmission Electron Microscopy}

\section{(i) RRV infected RhF}

$48 \mathrm{~h}$ p.i. media was removed from the $10 \mathrm{~cm}^{2}$ plate and RhF cells were washed 3 times with 1X PBS at RT. 2.5\% electron microscopy grade gluteraldehyde (Electron Microscopy Sciences) in 1X PBS was added to the plate to fix cells for

10 minutes at room temperature. Cells were then scraped into a tube and pelleted by centrifugation at $350 \times \mathrm{g}$ for 5 minutes at RT. The fixed cell pellet was stored at $4^{\circ} \mathrm{C}$ until processing at the University of Virginia (UVa) Advanced Microscopy (AVM) Core.

\section{(ii) Supernatants}

$48 \mathrm{~h}$ p.i. media was collected and concentrated by centrifugation over sucrose cushion as described above. Following centrifugation, media was decanted and the remaining pellet was fixed in $2.5 \%$ gluteraldehyde in $1 \mathrm{X}$ PBS. Samples were stored at $4^{\circ} \mathrm{C}$ until processing.

Samples were post-fixed in $2 \%$ osmium tetroxide, dehydrated in graded ethanol, and embedded in epoxy resin. Ultrathin sections, approximately 60$70 \mathrm{~nm}$ in thickness, were collected on 200 mesh copper grids and contraststained with lead citrate and uranyl acetate. The sections were examined at 80 kV on a JEOL 1230 transmission electron microscope at the UVa AVM Core. 


\section{Complementation of RRV ORF52 with exogenous siORF52 resistant plasmid}

N-terminal myc-tagged siORF52 resistant RRV ORF52 was generated from a pK-myc wt RRV ORF52 plasmid. The pK-myc plasmid was derived from pKH3 backbone and a gift from Deborah Lannigan and lan Macara. Full-length ORF52 sequence was amplified by PCR using purified RRV DNA as a template and primers that added a Notl and an EcoRI sequence to ORF52 for cloning (NotlORF52-F 5'-ATAAGAATGCGGCCGCTTATGTCTTCCACGCGT-3' and EcoR1ORF52-R 5'-GGAATTCCTAGTCCGCGTCGTTATT-3'). RRV ORF52 was cloned into the pK-myc vector at the Notl and EcoRI sites. The sequence of the wt pKmyc-RRV52 plasmid was confirmed prior to further use by sequencing with Sp6 Fwd primer and comparison to published RRV ORF52 genomic sequence. wt pK-myc RRV ORF52 plasmid was used as a template to generate an RRV ORF52 siRNA resistant plasmid (siORF52). Primers were designed that would alter 2 nucleotides (225 and 228) in the wobble base position of two adjacent amino acid coding sequences of ORF52 that are complementary to the ORF52 siRNA. Changed nucleotides are underlined. 5'AACCCGTAAAATCGAAGCTAA-3'. The siORF52 resistant plasmid was generated using the primers containing the desired mutations, the wt pK-myc ORF52 plasmid, and the QuikChange Lightening Site-Directed Mutagenesis Kit (Life Technologies). The mutations were verified by sequencing of the pK-mycsiORF52 resistant plasmid. 
Confirmation of Res52 expression and resistance to siORF52

$1 \times 10^{6}$ HEK293 cells in $2.5 \mathrm{~mL}$ media were plated in a 6 well plate containing 0.5 $\mathrm{mL}$ Opti-mem and $20 \mathrm{nM}$ siCNL or siORF52 and $5 \mu \mathrm{L}$ RNAiMax (Life Technologies). 24 hours later cells were transfected with $1 \mu \mathrm{g}$ pK-myc wtORF52 (wt52) plasmid or $1 \mu \mathrm{g}$ pK-myc-siORF52 resistant plasmid (Res52) combined with $3 \mu \mathrm{L}$ Lipofectamine 2000 (Life Technologies) in $500 \mu \mathrm{L}$ Opti-mem and subsequently incubated at $37^{\circ} \mathrm{C}$. 5 hours later media was removed, cells were washed with $1 \mathrm{X}$ PBS, and $3 \mathrm{~mL}$ fresh complete media was added. 48 hours later, media was removed and cells were washed 3 times in 1X PBS. Expression of ORF52 was determined by gel electrophoresis and western (methods are identical to those stated in the protein electrophoresis and immunoblotting and expression of viral lytic proteins section).

\section{Complementation assay}

$6 \times 10^{6} \mathrm{RhF}$ cells were transfected via Amaxa nucleofector program T-016 with 6 $\mu \mathrm{g}$ of pK-myc-empty vector or pK-myc-siORF52 resistant plasmid DNA in primary fibroblast nucleofection reagent and plated in a $10 \mathrm{~cm}^{2}$ plate. 24 hours later, cells were reverse transfected with siRNA by lifting cells off the plate with $0.05 \%$ trypsin and replating in $8 \mathrm{~mL}$ of complete media on $10 \mathrm{~cm}^{2}$ plates containing 2 $\mathrm{mL}$ Opti-Mem, $20 \mathrm{nM}$ siCNL or siORF52, and $25 \mu \mathrm{L}$ RNAiMax (Life Technologies). 24 hours later cells were infected in $2 \mathrm{~mL}$ complete media for 1 hours with RRV at an $\mathrm{MOI} 5$ at $37^{\circ} \mathrm{C}$ and rocked every 15 minutes to ensure even distribution of virus. Following infection, virus was removed, cells were 
washed 3 times in 1 X PBS, and complete media was added. Media and cells were collected $48 \mathrm{~h}$ p.i. and protein composition determined by gel electrophoresis and western (methods for determining cell pellet and protein composition in the media are identical to those stated in the protein electrophoresis and immunoblotting and expression of viral lytic proteins section).

\section{IF Assay for Complementation}

$6 \times 10^{6} \mathrm{RhF}$ cells were transfected via Amaxa nucleofector program T-016 with 6 $\mu \mathrm{g}$ of pK-myc-empty vector or pK-myc-siORF52 resistant plasmid DNA in primary fibroblast nucleofection reagent and incubated in a $10 \mathrm{~cm}^{2}$ plate for 24 hours. 25,000 cells/well were subsequently reverse transfected with RNAiMax (Invitrogen) with either ORF52 siRNA or control siRNA and plated onto 48 well plates containing a Cell-Tak (BD Biosciences) coated $8 \mathrm{~mm}$ round coverslip (Electron Microscopy Sciences) and incubated for 24 hours. Cells were then infected with RRV at a $\mathrm{MOI}$ of 5 or mock infected. Remainder of staining and imaging methods are the same as above in IF Assay, except the secondary antibodies used were Alexa Fluor 488 goat anti-mouse and Alexa Fluor 555 goat anti-rabbit (both 1:500) (Life Technologies).

\section{Results}

Efficient knockdown of ORF52 has little effect on other viral structural proteins 
To begin investigating the function of ORF52 during lytic RRV infection, we first knocked down its expression by transfecting RhFs with either control (siCNL) or ORF52 (siORF52) specific siRNA 24 hours prior to the addition of the virus. Using quantitative western blots, we then measured the relative levels of ORF52 as well as several other lytic structural proteins in the cell lysates (Figure 2-1A and $2-1 B$ ). In cells targeted with ORF52 siRNA, the knockdown of ORF52 was nearly complete at $97 \%$ compared to the robust expression in controls (siCNL). In contrast, another tegument protein, ORF45, and capsid proteins, small capsomer interacting protein (SCIP/ORF65) and major capsid protein (MCP/ORF25), showed no statistically significant change (Figure 2-1B). We noted similar results with the use of a second ORF52-targeting siRNA (data not shown). These results suggested that expression of the structural proteins we examined were independent of the synthesis of ORF52 and that the knockdown was specific.

\section{Loss of ORF52 markedly decreases viral titer}

We next determined if the depletion of ORF52 had an effect on productive RRV infection by assessing the release of infectious virions. In earlier work with MHV68 , cells transfected with a BAC construct containing a stop codon in its ORF52 (ORF52Stop-BAC) did not result in the release of measurable infectious virus (27). Therefore, we hypothesized that if ORF52 were likewise required for RRV production then the titers we measured from siORF52 treated cells would be significantly lower than from controls. In five separate experiments, we found 
Figure 2-1. Efficient knockdown of ORF52 significantly decreased RRV titer with only minimal effects on intracellular levels of other structural viral proteins. (A) Immunoblot analysis of cell lysates from RhF transfected with control siRNA (siCNL), lane 1, or ORF52 siRNA (siORF52), lane 2 and then infected with RRV 24 hours later at MOI 5. Cells were harvested 48 h p.i. and immunoblots were performed, probing for the viral tegument proteins, ORF52 and ORF45, the capsid proteins, MCP and SCIP, and, in addition, actin, to normalize for loading differences. (B) Graphical representation of the intracellular levels of the indicated viral structural proteins after siORF52 treatment relative to siCNL in RRV infected RhF cells. Data represent the mean +/- SD of 6 individual experiments. (C) Viral titers in the media $48 \mathrm{~h}$ p.i. from $\mathrm{RhF}$ treated with siCNL or siORF52 were determined from 6 different experiments by viral plaque assay. Values are mean $+/-$ SD. ${ }^{* * *} p<0.0001$ 

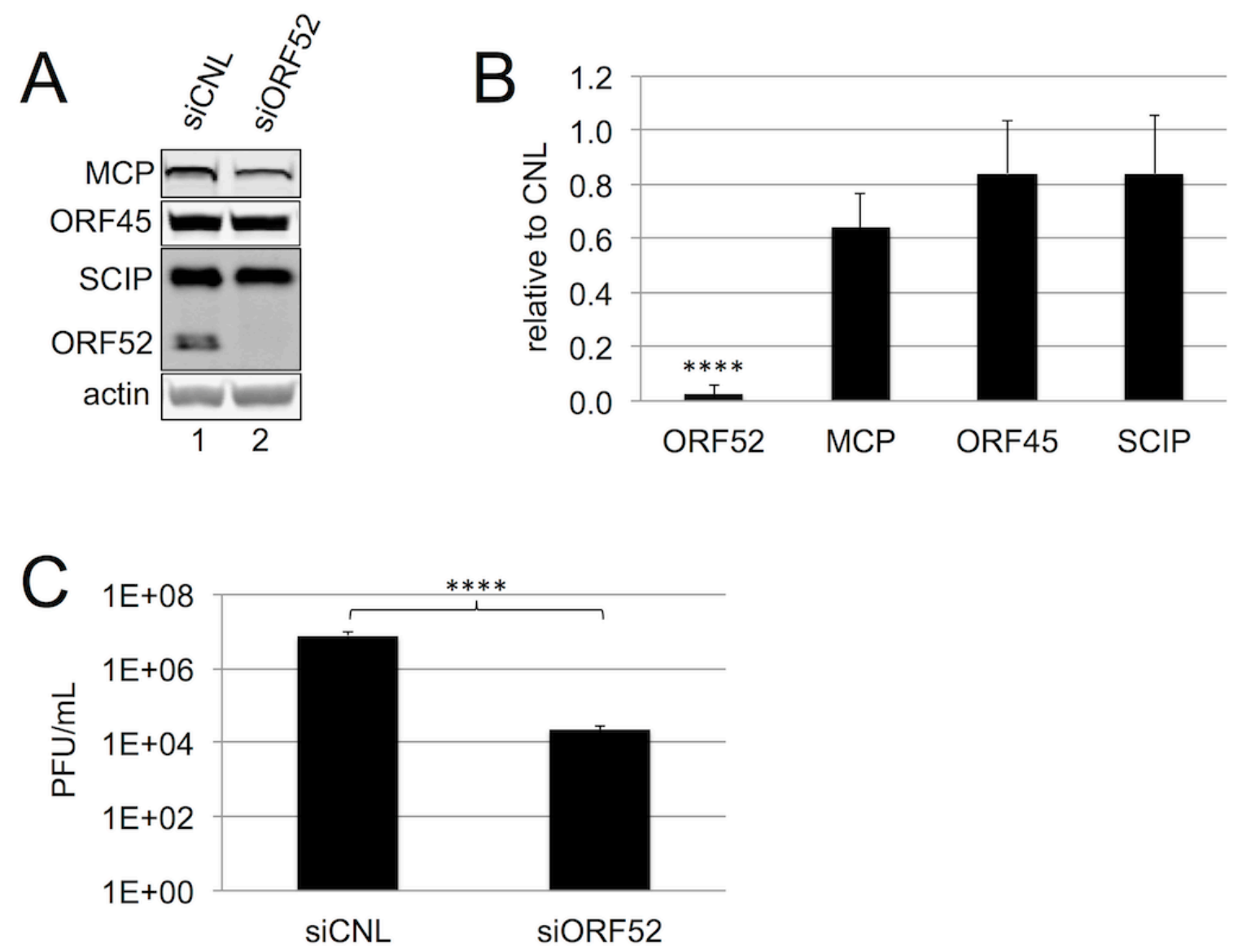
that the titers from siORF52 treated cells were over 300 -fold lower than from controls (Figure 2-1C), indicating that ORF52 was indeed critical for efficient production and/or release of infections virions.

We subsequently asked whether depletion of ORF52 affected intracellular levels of viral DNA, thereby leading to the decrease in viral titer we observed following ORF52 knockdown. Since viral protein production of lytic proteins seemed grossly unaffected despite the near absence of ORF52 (Figure 2-1A and 2-1B) and since ORF52 is a late gene expressed after viral DNA replication (73), we hypothesized that viral DNA copies would not be markedly affected in siORF52 treated cells. We, instead, predicted that the block in virus production would be at a stage after viral DNA replication. QPCR analysis showed that viral DNA increased approximately 3 orders of magnitude between 12 and 24 hours for siCNL treated cultures following infection and only modestly less with ORF52 knockdown (Figure 2-2A). While DNA in cell lysates with ORF52 knocked down had $25 \%$ and $50 \%$ fewer copies of viral DNA at 24 and 48 hours post-infection, respectively, the overall kinetics and expression profile closely paralleled that of siCNL treated cells, suggesting that knockdown of ORF52 did not grossly inhibit viral DNA replication (Figure 2-2A). Combined, these results indicated that neither the production of structural viral proteins (Figure 2-1A and 2-1B) nor the lytic replication of viral DNA (Figure 2-2A) could account for the profound reduction (>300-fold) in viral titer that resulted from ORF52 knockdown. Rather, the findings suggested a block at a subsequent stage of particle maturation. 
Figure 2-2. Intracellular viral DNA production and packaging within particles appeared independent of ORF52. (A) RhF cells were transfected with siCNL or siORF52 and 24 hours later infected with RRV at MOI 5. At the time points indicated, cells were collected and total intracellular DNA was isolated and purified. Viral DNA was quantified by SYBR green qPCR with primers to the RRV ORF45 coding region. (B) RhF cells were transfected with siCNL or siORF52 and 24 hours later infected with RRV at MOI 5. At the time points concentrated particles were treated with DNase followed by PK and viral DNA was isolated, purified, and quantified by SYBR green qPCR with primers for RRV ORF45. Values are the mean and SD of 2-3 qPCR replicates from 3 separate experiments with only the positive component of errors shown for increased graphical clarity. 


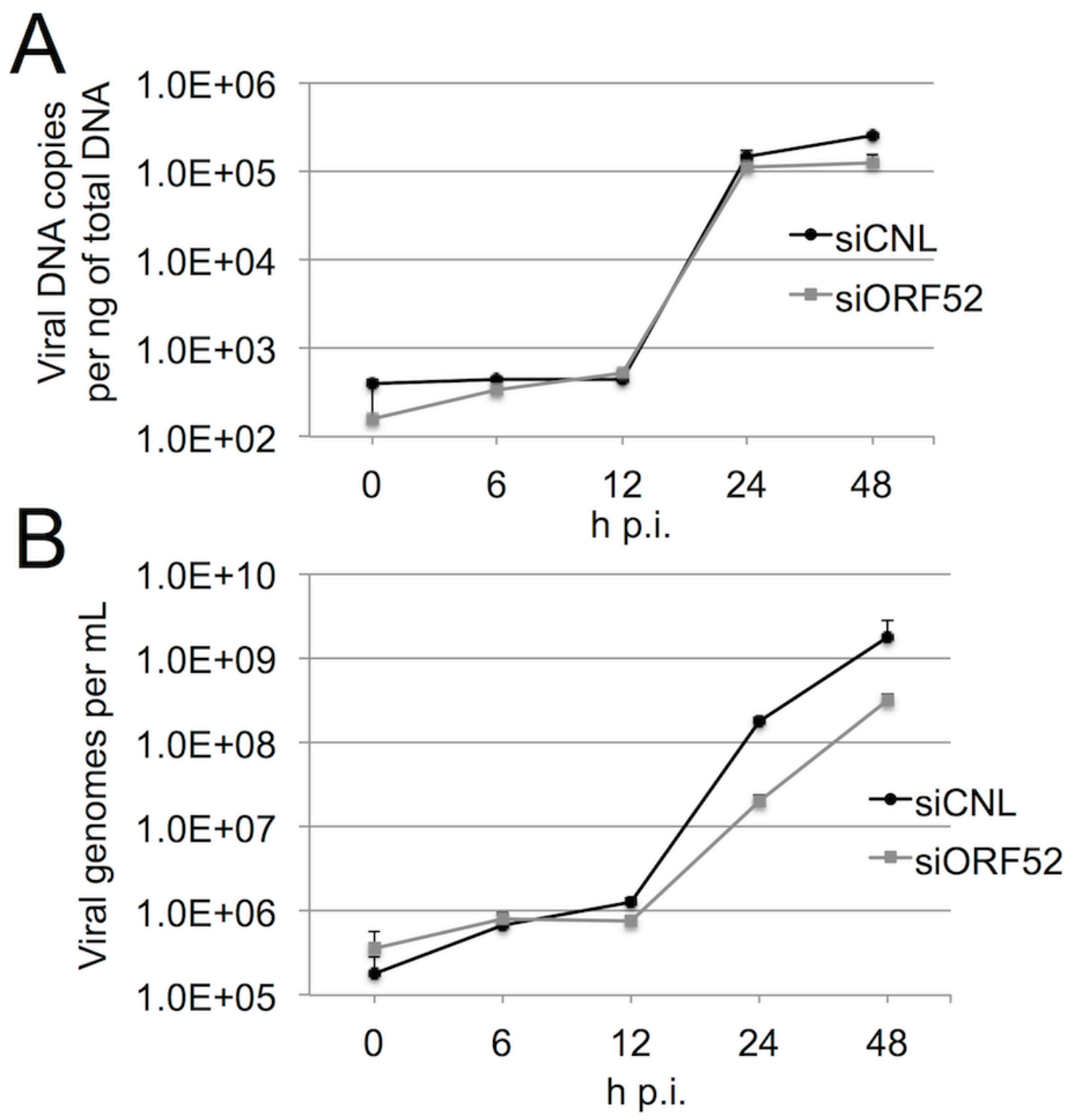




\section{Release of an increased proportion of empty particles}

Although intracellular viral DNA and lytic structural proteins were present at close to control levels despite the near absence of ORF52, we could not rule out the possibility that ORF52 might be necessary for DNA packaging of capsids within the nuclei. Therefore, we next asked whether ORF52 knockdown led to a decrease in the DNA content of released particles, measuring encapsidated viral DNA in media from cells treated with siCNL or siORF52 at multiple time points following infection with RRV. DNAse-resistant (encapsidated) viral DNA in the media of both siCNL and siORF52 treated RRV infected cells began to appreciably accumulate $12 \mathrm{~h}$ p.i. and continued to increase through $48 \mathrm{~h}$ p.i. (Figure 2-2B). However, encapsidated viral DNA released from siORF52 treated cells was approximately 9 -fold and 6 -fold lower at 24 and 48 h p.i., respectively, compared to control levels at these times (Figure 2-2B). These results suggested that ORF52 knockdown led to the release of either fewer overall particles or a lower proportion of particles containing viral DNA, or both. In either case, however, the modest difference in encapsidated viral DNA at $48 \mathrm{~h}$ p.i. in the media from the ORF52 knockdown (kd) condition was also insufficient to explain the large drop in titers (>2.5 orders of magnitude) (Figure 2-1C). Therefore, it followed that the major contributor to the loss in titer likely stemmed from a defect in particle infectivity other than DNA packaging or drop in particle number.

\section{ORF52 knockdown led to the release of subviral particles lacking tegument}

To test whether the decrease in encapsidated viral DNA drop from ORF52 
knockdown resulted from a concomitant drop in released particles, we first collected media from siCNL or siORF52 treated RhF cells $48 \mathrm{~h}$ p.i., isolated potential viral particles from the media by centrifugation through a sucrose cushion and then used quantitative immunoblotting to measure the levels of particle-associated structural proteins from equal volumes of concentrated media. Since ORF52 knockdown led to such a dramatic decrease in viral titer that was nearly 2 orders of magnitude greater than the relatively modest reduction in encapsidated viral DNA, we predicted that we would observe qualitative as well as quantitative differences in the protein composition of the released particles. As we anticipated, siCNL treatment of RhF cells led to the production of particles containing capsid proteins MCP and SCIP, as well as tegument proteins ORF45 and ORF52 (Figure 2-3, lane 1). The particles from siORF52 treated cells also contained the capsid proteins MCP and SCIP and, not surprisingly, they lacked ORF52. We noted, however, that the difference in particle associated capsid proteins (MCP and SCIP) released from siCNL and siORF52 treated cells was minimal (Figure 2-3B). Since the stoichiometry of both SCIP and MCP is fixed in the icosahedral capsid of all herpesviruses $(121,133$, $226,299)$, these results suggested that the overall abundance of released virion or subvirion-like particles was similar in the two conditions following RRV infection.

In contrast, we noted that the released particles from the ORF52 kd conditions also lacked the tegument protein ORF45, despite its presence within the siORF52 treated cell lysates (Figure 2-1A and 2-1B). Figure 2-3B depicts 
Figure 2-3. In the absence of ORF52, RRV infection led to release of immature particles lacking tegument. (A) RhF cells were transfected with siCNL, lane 1, or siORF52, lane 2, and then infected 24 hours later with RRV at MOI 5. 48 h p.i., supernatants were collected and concentrated over a $20 \%$ sucrose cushion to isolate particles and equal volumes of media were separated by SDS-PAGE and immunoblotted for MCP, ORF45, SCIP, and ORF52. (B) Graphical representation of the effect of siORF52 relative to siCNL on the levels of the indicated viral protein within the released viral particles. Data represent the mean $+/-$ SD of 6 individual experiments. ${ }^{* *} p<0.01$ and ${ }^{* * *} p<0.0001$. 

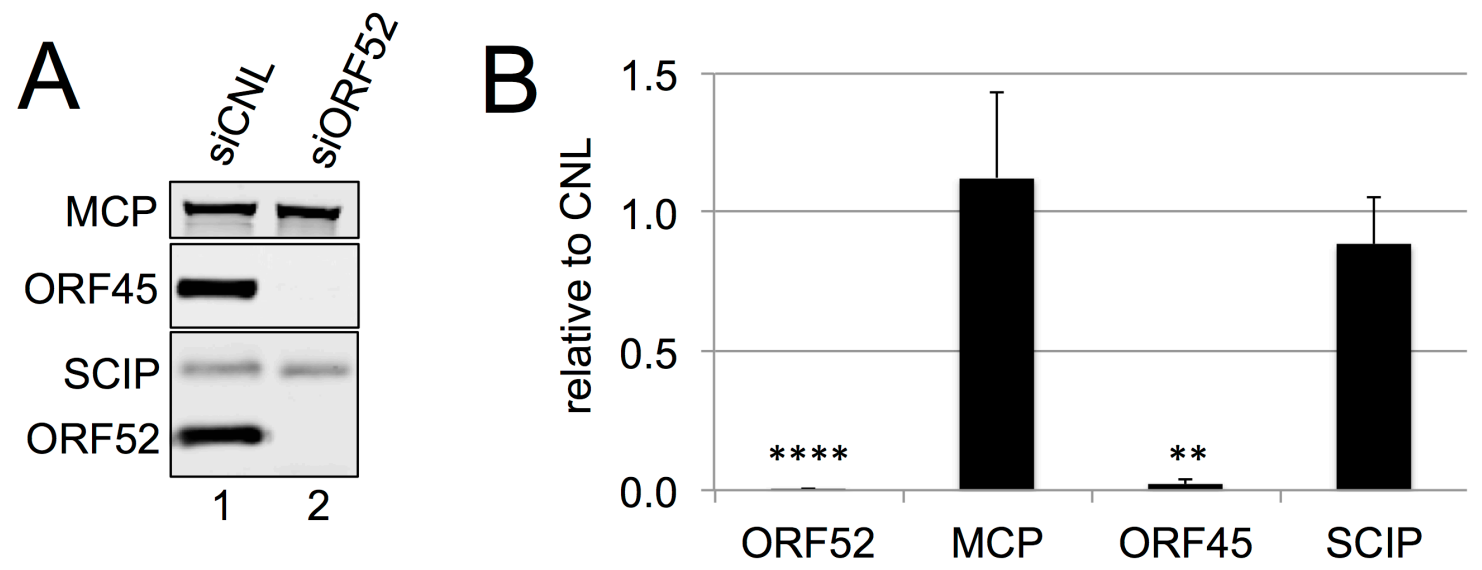
graphically the protein levels from six separate experiments quantifying the ratio of each protein associated with particles released from siORF52 compared to siCNL treated cells. These results demonstrated that both ORF52 and ORF45 were virtually absent in particles present in the media of siORF52 treated cells. Since the decrease in viral titer between siORF52 compared to siCNL treated cells was 300-fold (Figure 2-1C), despite the suggestion of similar particle numbers (Figure 2-3B), we reasoned that the greatly lower infectivity of particles in the media from ORF52 knockdown cells likely resulted from the production of incomplete particles lacking, at a minimum, a full complement of tegument proteins.

\section{Loss of ORF52 prevents ORF45 incorporation into the particle}

As we have just remarked above, though the intracellular abundance of tegument protein ORF45 remained essentially unaffected following ORF52 knockdown (Figure 2-1A and 2-1B), it was absent from released particles (Figure 2-3A and 23B). Earlier studies with KSHV have documented that KSHV ORF45 and KSHV ORF52 interact by both co-IP and yeast two-hybrid studies, though the latter was only evident when ORF52 served as bait but not as prey (267) and similar interactions are less clear for the murine gammaherpesvirus, MHV-68 (82, 330). To gain further insight into the interactions between ORF52 and ORF45 during RRV infection, we assessed their subcellular localization using immunofluorescence microscopy (IF) in the absence or presence of ORF52 knockdown (Figure 2-4). In siCNL cells, IF revealed that ORF45 localization was 
diffuse in the nucleus with nucleolar sparing but highly punctate in the cytoplasm while ORF52, in agreement with MHV-68 ORF52 staining (27), displayed a discrete punctate pattern in the cytoplasm and was absent from the nucleus. Additionally, the merge of the two staining patterns demonstrated that punctate patterns of ORF52 and ORF45 in the cytoplasm overlapped, consistent with their representing individual or collections of viral or subviral tegumented particles (Figure 2-4A, upper panels and 2-4B, left panel). To help assess whether the punctate cytoplasmic staining of the two tegument proteins did reflect maturing virions, we also co-stained for SCIP, reasoning that it would localize to trafficking capsids and would co-localize with both ORF52 and ORF45. However, we found that cytoplasmic compared to nuclear SCIP staining was weak, possibly reflecting a partial masking of the SCIP epitope due to the overlying tegument proteins. In contrast, following ORF52 knockdown, ORF45 remained strictly nuclear and SCIP staining in the cytoplasm was now prominent, though mainly adopting an aggregated perinculear distribution (Figure 2-4A, lower panels and 2-4B, right panel). Together, these images suggested a role for ORF52, directly or indirectly, in the export of ORF45 into the cytoplasm. Of note, co-IP experiments failed to demonstrate an interaction between ORF45 and ORF52 either from RRV infected RhF cells or from HEK293 cells following ectopic expression of the two tegument proteins (data not shown). Thus, during unperturbed assembly of the virions, ORF45 appeared to exit the nucleus by its incorporation within maturing particles as they acquired tegument. The exact mechanism underlying the ORF52-dependence of this incorporation, however, 
Figure 2-4. ORF52 knockdown restricted ORF45 to nucleus. (A) RhF cells were reverse transfected with siCNL, upper panels (i-v), or siORF52, lower panels (vi-x) and plated onto coverslips. 24 hours later cells were infected with RRV at MOI of 5 and then $48 \mathrm{~h}$ p.i., cells were fixed and stained with conjugated anti-SCIP, anti-ORF52, and anti-ORF45 antibodies, as indicated, followed by the secondary antibodies Alexa Fluor 647 goat anti-mouse or Alexa Fluor 555 goat anti-rabbit. Cells were stained with DAPI. (B) Magnified (3X) areas indicated by dotted lines in merged images of $(A)$, panels $v$ and $x$. Arrows in siCNL (panel i) point to punctate orange staining (due to ORF45-ORF52 co-localization) in the cytoplasm, consistent with tegumented viral particles during maturation/egress. Arrowheads in siORF52 (panel ii) point to yellow cytoplasmic staining consistent with SCIP Ab reactivity in the absence of ORF45 and ORF52 reactivity and suggesting untegumented cytoplasmic capsids. 


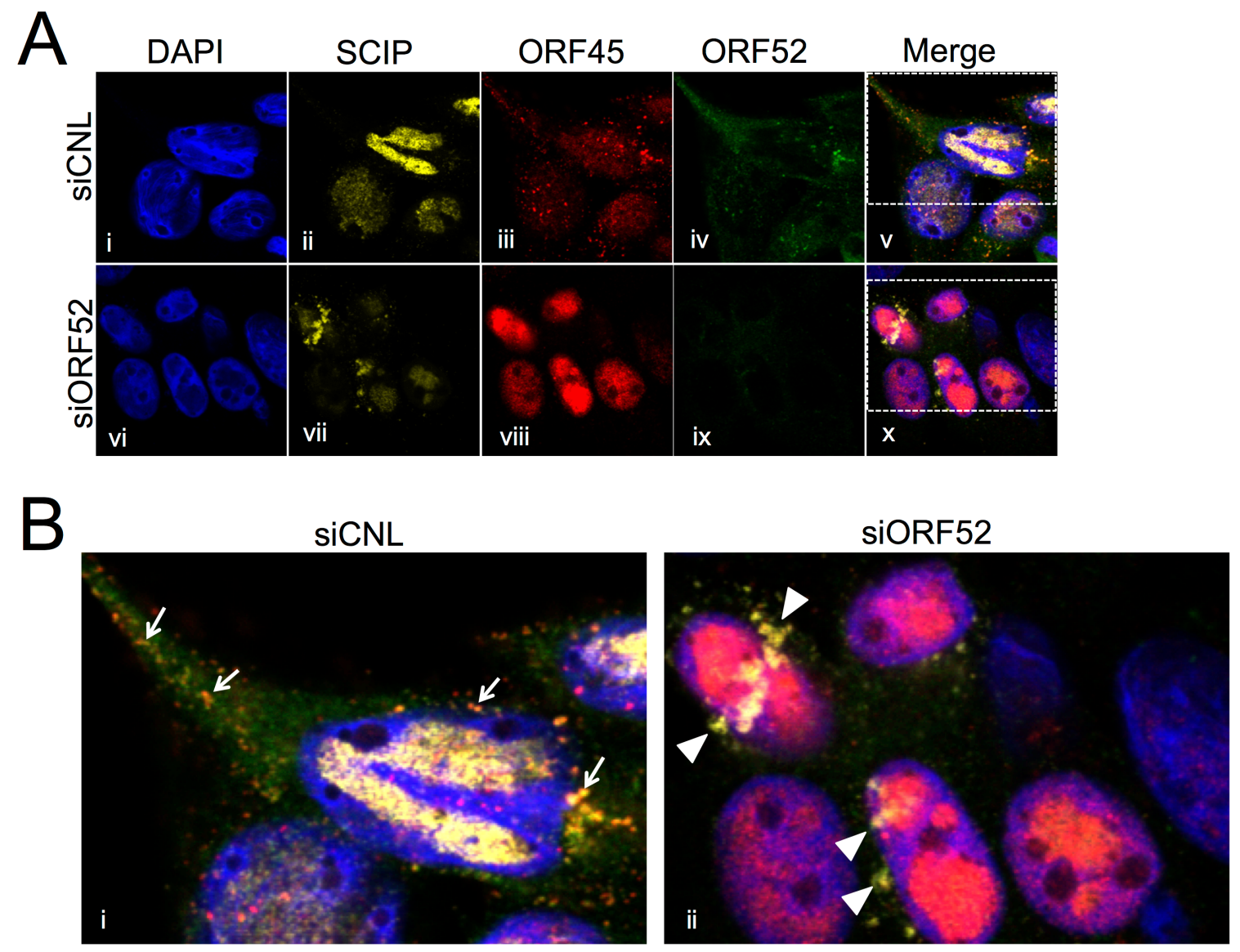


remains unclear (see Discussion).

\section{Secondary envelopment depends on ORF52}

Since the immunofluorescence images of infected cells with ORF52 knockdown showed little to no evidence of either ORF52 or ORF45 in the cytoplasm, and immunoblots of the released particles from these cells, likewise, demonstrated an absence of these two tegument proteins, we hypothesized that the particles would more closely resemble capsids rather than mature trilaminar virions. Further, since we also detected lower levels of encapsidated DNA in the media from ORF52 kd cells, we anticipated observing a greater proportion of empty particles lacking electron-dense genomic viral DNA otherwise typical in electron micrographs of mature herpesviruses $((36,184,349,356)$ and reviewed in $(208$, $211,213))$

After centrifuging media over a sucrose cushion, we fixed the pelleted samples and visualized any particles from both ORF52 knockdown and control conditions using thin-section transmission electron microscopy (TEM). The majority of particles from siCNL treated cells appeared to be mature virions containing DNA, an icosahedral capsid, tegument, and envelope (Figure 2-5A). In contrast, the particles from siORF52 treated cells had the shape and diameter of capsids and lacked evidence of either tegument or envelope (Figure 2-5B). A number of these subviral particles contained the electron-dense DNA that is typical of TEM images of herpesviruses; however, compared to siCNL, there was an increase in the proportion of empty (lacking DNA) capsids present 
Figure 2-5. TEM images of the concentrated media from RRV-infected RhF after ORF52 knockdown demonstrated release of untegumented and unenveloped capsid-like particles, many of which lacked DNA. RhF cells were transfected with siCNL or siORF52 and infected 24 hours later with RRV at MOI 5. $48 \mathrm{~h}$ p.i. media was removed and layered over a sucrose cushion prior to centrifugation. Pelleted supernatants were fixed and examined by TEM at $30 \mathrm{~K}$ magnification. In contrast to the siCNL sample (A) that contained mature virions with envelope (e) and tegument (t) surrounding a capsid (c), particles from siORF52 treated RhF cells (B) appeared to lack both envelope and tegument. (d), encapsidated viral DNA. Inset images are expanded 3X from original images (dashed boxes). 

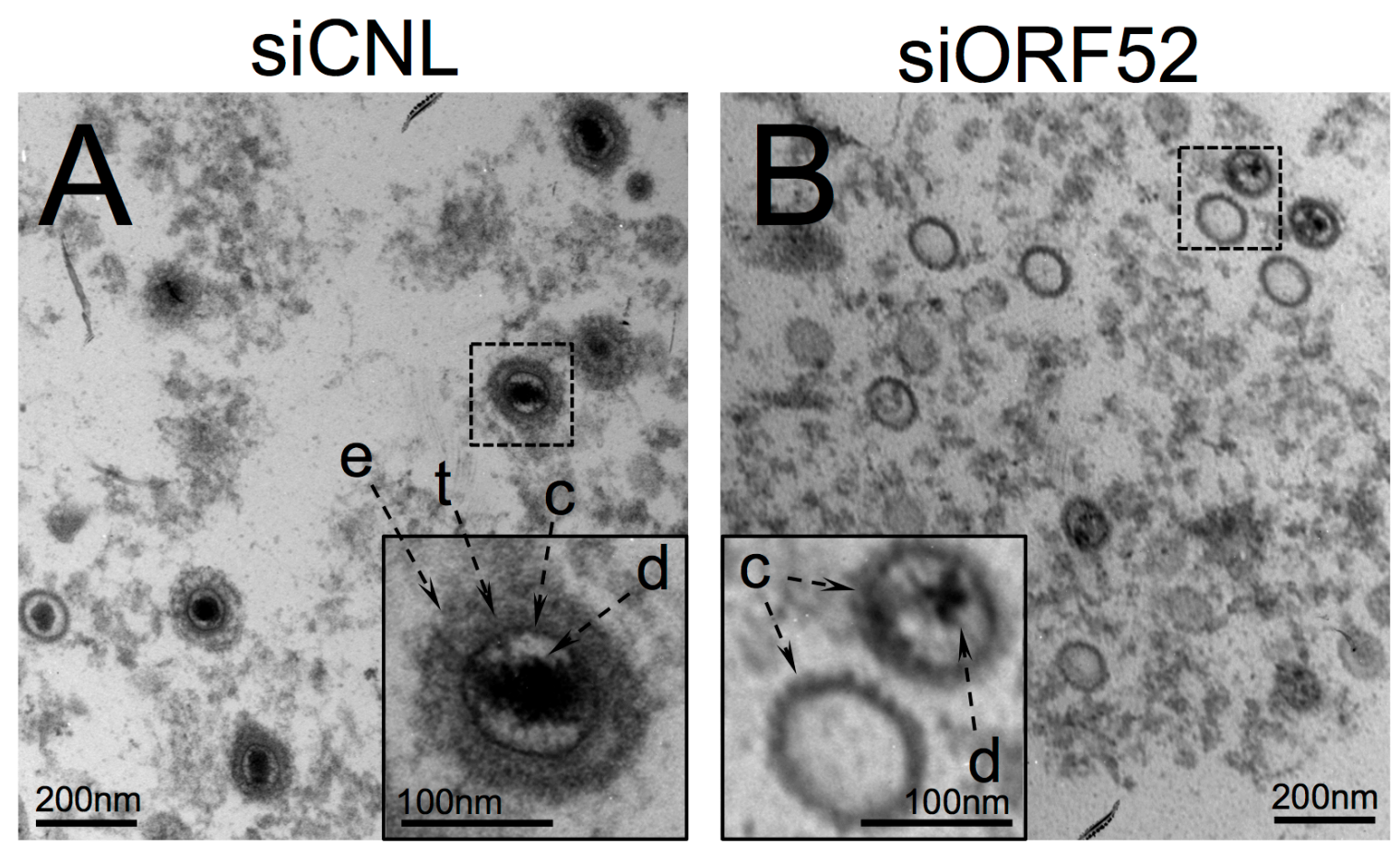
(Figure 2-5B). This result suggested that ORF52 was necessary for the production and release of fully formed virions at a stage subsequent to capsid protein production, assembly and DNA replication and packaging but before tegumentation and envelopment.

\section{ORF52 is necessary for tegumentation and envelopment}

Although we had anticipated that knockdown of a conserved tegument protein such as ORF52 might interfere with virion maturation, we also expected that this block would inhibit particle release. Thus, we were struck by the apparent efficiency with which infected cells knocked down for ORF52 still released subviral (defective) particles in quantities that approximated the numbers of presumably mature virions from control cells (Figure 2-3B). To better characterize this unexpected phenomenon and to more precisely elucidate the stage that requires ORF52 for virion maturation, we used TEM to examine the morphology and distribution of particles within infected cells with or without ORF52 knock down.

That ORF52 appeared to play a critical structural role in tegumentation and secondary envelopment, similar to its homolog in MHV-68 (27), was consistent with its cytoplasmic localization during lytic infection (Figure 2-4) and our earlier finding that it belongs to the inner tegument of RRV (239). TEM revealed that cells receiving either siCNL or siORF52 produced all three major types of capsid species within their nuclei, which is typical for infection with RRV and other herpesviruses $((110,121,226,238,299)$ and reviewed in (133)): A, 
empty; B, containing scaffold protein; and C containing viral DNA (Figure 2-6A and 2-6B). These results demonstrated that capsid assembly and DNA packaging did not require ORF52 expression and provided strong support for our earlier data showing that released particles still had DNase-resistant, packaged viral DNA, even after kd of ORF52 (Figure 2-2B).

Likewise, we noted that capsid egress from the nucleus into the cytoplasm also appeared independent of ORF52 expression. TEM revealed cytoplasmic particles under both knockdown and control conditions (Figure 2-6C and 2-6D). In the cytoplasm of siCNL treated cells, multiple trilaminar, mature virions were evident within large vesicles (Figure 2-6C). However, in cells treated with siORF52, the cytoplasm contained a large number of untegumented and unenveloped subviral particles that were indistinguishable from nuclear capsids and no evidence of mature virions (Figure 2-6D).

Since invagination into vesicles appears coupled with tegumentation and secondary envelopment (reviewed in (211)), it seemed that ORF52 was necessary for these maturation steps (Figure 2-6D). We also noted that in ORF52 knockdown cells there appeared to be a larger number of total capsids present in the cytoplasm compared to the combined number of subviral particles and virions in siCNL cells (quantified below), providing further evidence that inhibition of tegumentation in cells lacking ORF52 created a block in efficient release of particles via the canonical vesicle-based pathway. Comparison of particles from the nucleus of siCNL or siORF52 treated cells and the cytopasm of siORF52 treated cells showed that the species appeared similar in size and 
Figure 2-6. TEM of RRV infected cells following ORF52 knockdown showed block in tegumentation and secondary envelopment, leading to perivesicular accumulation of capsids as well as a lack of cell surfaceassociated virions. RhF cells were transfected with siCNL or siORF52 and infected as in Figure 5. $48 \mathrm{~h}$ p.i. cells were fixed and examined by TEM at $40 \mathrm{~K}$ magnification. The nucleus of siCNL (panel A) and siORF52 (panel B) treated cells contained A (empty) capsids (unfilled arrowhead), B capsids containing scaffold (chevron), and C capsids with DNA (arrow). The cytoplasm of siCNL cells (panel C) contained large vesicles (ves) filled with multiple tegumented and enveloped mature virions. In contrast, cytoplasm of siORF52 treated cells (panel D) contained untegumented, unenveloped capsids that were juxtaposed to but not within vesicles (filled arrowheads indicate capsids surrounding vesicle). Multiple extracellular plasma membrane $(\mathrm{pm})$-associated virions were present in siCNL cells (panel E) but not in siORF52 samples (panel F). Inset images are expanded $3 X$ from original images. Cyto (cytoplasm), nuc (nucleus). 

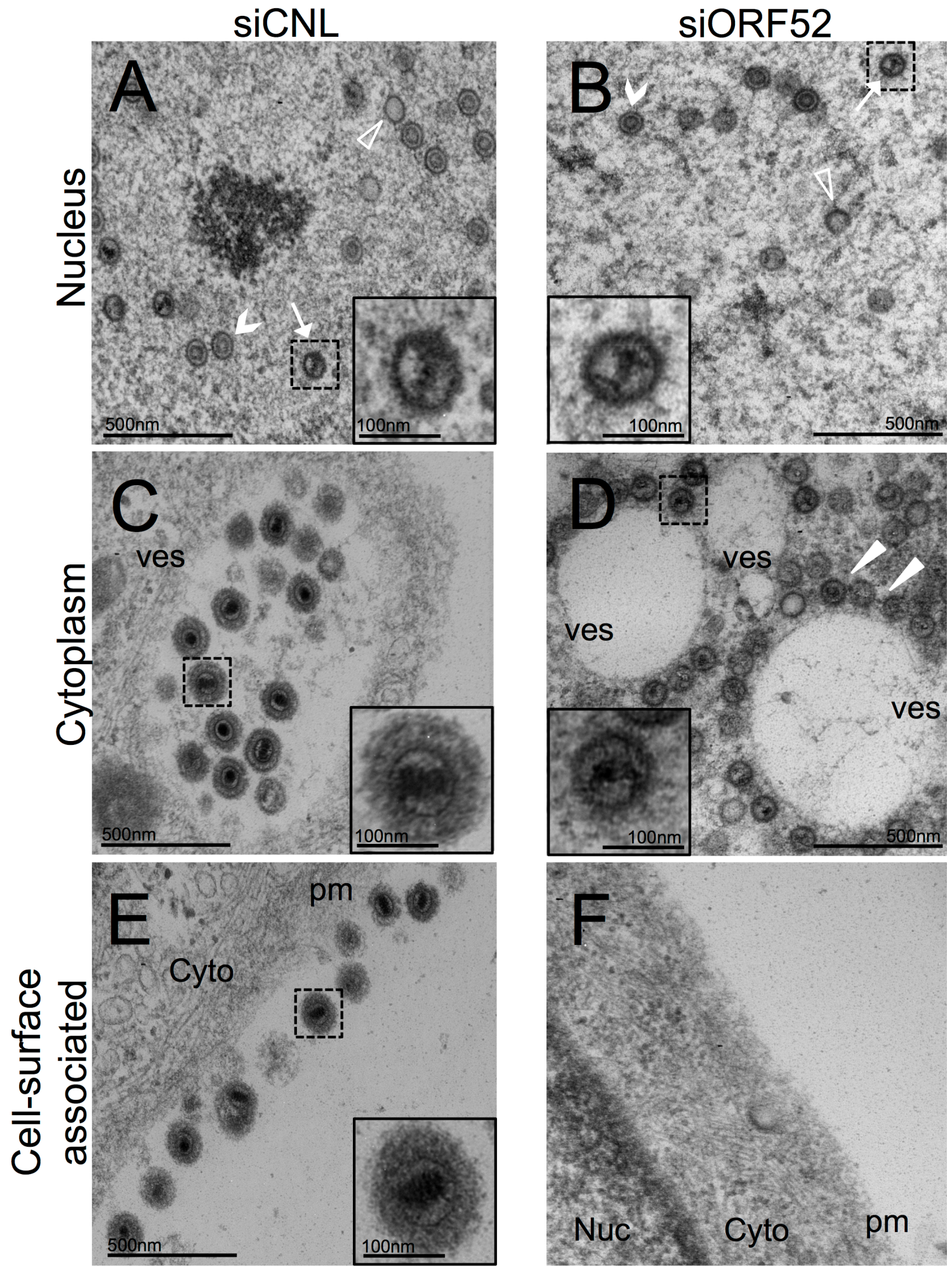
morphology (compare insets from Figures $2-6 \mathrm{~A}$ and 2-6D). This finding suggested the particles in the cytoplasm of cells knocked down for ORF52 likely lacked most, if not all, of their tegument as well as their envelope.

\section{Efficient release and cell-surface association of viral particles are ORF52 dependent}

The presence of cell surface associated virions post egress is typical during productive infection with herpesviruses. Although the mechanism of particle release into the media from ORF52 knockdown cells remains unclear, we predicted that these subviral particles, lacking tegument and envelope, would also be defective in adhering to the cell surface since in other herpesviruses (and enveloped viruses, in general) this depends on the interaction between cellular BST2/tetherin and viral envelope glyocproteins ((146, 150, 192, 244, 271, 351) and reviewed in (324)). To address this question, we examined the cell surface of both siORF52 and siCNL treated cells and found that knockdown of ORF52 led to a marked absence of cell surface associated particles (Figure 2-6F). In comparison, control cells showed mature virions populating large areas of the plasma membrane (Figure 2-6E).

To assess more precisely the effect of ORF52 on viral maturation, we quantified the different phenotypes of particles within the nuclear and cytoplasmic compartments, as well as those released particles collected from the media (Table 2-1). While the total number of particles in siCNL and siORF52 was similar, overall, the relative proportion of the different viral and subviral species 
Table 2-1. Distribution of intracellular and released viral and subviral particles following RRV infection with and without ORF52 knockdown 


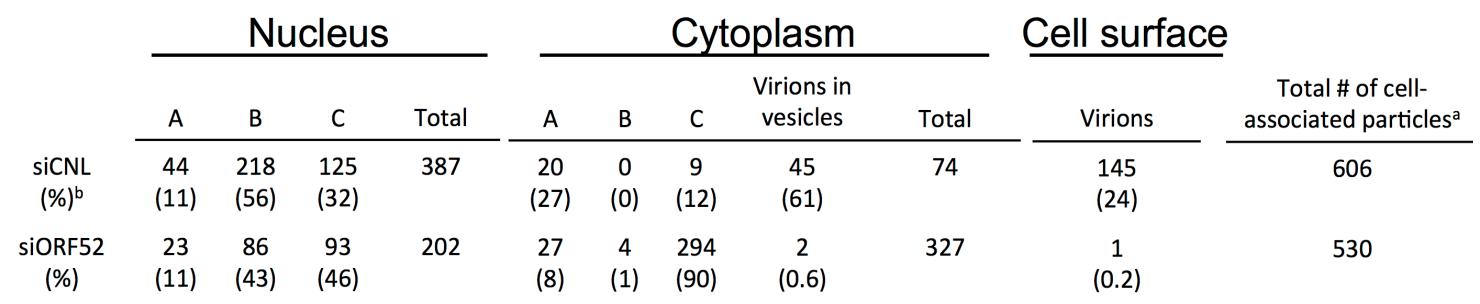

\begin{tabular}{cccccc} 
& \multicolumn{5}{c}{ Media } \\
\cline { 2 - 5 } & A & B & C & Virions & $\begin{array}{c}\text { Total \# of particles } \\
\text { counted }\end{array}$ \\
\cline { 2 - 6 } siCNL & 43 & 9 & 32 & 147 & 231 \\
$(\%)$ & $(19)$ & $(4)$ & $(14)$ & $(63)$ & \\
siORF52 & 176 & 32 & 95 & 2 & 305 \\
$(\%)$ & $(58)$ & $(10)$ & $(31)$ & $(0.7)$ &
\end{tabular}

aTotal particles in TEM images from $10 \mathrm{RhF}$ cells transfected with siNCL or siORF52 for 24 hours and then infected at MOI 5 for 48 hours.

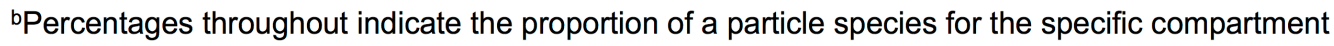
except for Cell Surface which reflects the proportion particles in all compartments. 
and their location differed markedly between control and knockdown

conditions. In siCNL cells, the nuclei contained approximately twice as many capsids as in the ORF52 knockdown; however, the proportion of $A, B$, and $C$ capsids were similar. Conversely, in the cytoplasm, cells lacking ORF52 contained over four times as many particles as were present in siCNL cells, suggesting that egress from the cell was less significantly efficient. We reasoned that this relative intracellular accumulation of particles might have led to fewer released particles in the media of the kd compared to control condition; however, we found little difference (Figure 2-3B). One possible explanation for this is that a large portion of the particles following egress in the controls remained cellsurface associated (see Figure 2-6F), thereby lowering the number released into the media.

In siCNL cells, virions within vesicles constituted the majority (61\%) of total cytoplasmic particles while in siORF52 cells, virions within vesicles totaled only 2 of the $327(<1 \%)$ viral particles that we detected. Unenveloped and untegumented capsids comprised the remaining cytoplasmic subviral particles in the siORF52 treated cells and were often present surrounding vesicles as evident in Figure 2-6D (see arrowheads). Examining 10 randomly selected infected cells from siCNL and siORF52 treatments, we enumerated the cell surface-associated virions near the cellular plasma membrane and detected 145 cell surface-associated virions in siCNL cells in contrast to 1 subviral particle evident in siORF52 conditions. Combined with the TEMs (Figures 2-5 and 2-6), these results demonstrated that ORF52 played little if any role in capsid 
formation, DNA packaging, or nuclear egress, but, instead, was critical for later steps of maturation, including tegumentation, secondary envelopment, release and virion adherence to the cell surface.

\section{Expression of siRNA-resistant ORF52 in trans partially rescued maturation and release of infectious virions in ORF52 knockdown cells}

To confirm that the block in tegumentation and secondary envelopment was specific to ORF52 knock down and not to off-target effects, we sought to rescue the defect by providing siRNA-resistant myc-tagged ORF52 in trans (Res52). We engineered the Res52 plasmid to have two wobble base changes, making it resistant to siORF52. To confirm that Res52 was expressed in cells and resistant to siORF52, we first transfected HEK293 cells with no siRNA, siCNL, or siORF52 and 24 hours later transfected cells with wt ORF52 (wt52) or Res52. Cells transfected with wt52 and Res52 alone, or with siCNL, expressed ORF52 (Figure 2-7A, lanes 1, 2, 4, and 5). Cells treated with siORF52 and transfected with wt52 effectively knocked down ORF52 (Figure 2-7A, lane 3), in contrast to cells that received Res52 that still expressed ORF52 even with siORF52 transfection (Figure 2-7A, lane 6). We next transfected RhF cells with either empty myctagged vector (pk-myc) or Res52 and 24 hours later treated the cells with siCNL or siORF52 and then infected at an $\mathrm{MOI}$ of 5 as we had in our earlier experiments. Infection of RhF cells transfected first with an empty-myc tagged vector (pk-myc) and siCNL expressed MCP and SCIP, as well as ORF45 and ORF52 (Figure 2-7B, lane 1), whereas cells transfected with pk-myc and 
Figure 2-7. Complementation of ORF52 knockdown with exogenous siRNAresistant ORF52 partially rescued wild-type phenotype. (A) HEK293 cells were reverse transfected with siCNL or siORF52. 24 hours later cells were transfected with wt myc-tagged ORF52 (wt52) or siORF52 resistant myc-tagged ORF52 (Res52) and harvested for immunoblot analysis 48 hours later. (B-F) RhF cells transfected with either empty myc-tagged vector $(\mathrm{Vec})$ or Res52 were reverse transfected 24 hours later with siCNL or siORF52. After an additional 24 hours cells were infected with RRV at MOI of 5 and then harvested $48 \mathrm{~h}$ p.i. (B) Immunoblot analysis of cell lysates from infected RhF first transfected with siCNL + Vec, lane 1, siORF52 + Vec, lane 2, or siORF52 Res52, siORF52 + Res52, lane 3. Protein blots were incubated with Abs specific for the viral tegument proteins ORF45 and ORF52, the capsid proteins, MCP and SCIP, and cellular actin, to normalize for loading differences. (C) Graphical representation of the levels of the indicated viral proteins after siORF52 treatment with Vec (black bars) or Res52 (gray bars) relative to siCNL in infected RhF cells. Data represent the mean $+/-$ SD of 4 individual experiments. (D) Immunoblot analysis of concentrated media collected from RhF transfected siCNL + Vec, lane 1, siORF52 + Vec, lane 2, or siORF52 + Res52, lane 3. Protein blots of the supernatants were probed for ORF45, ORF52, MCP, and SCIP. (E) Graphical representation of the levels of the indicated viral proteins in released particles after siORF52 treatment following transfection with Vec (black bars) or Res52 (gray bars), relative to those in particles released from siCNL treated cells. Data represent the mean and SD of 4 individual experiments. For $(C)$ and $(E) p$ values are calculated comparing knockdown (black bar) to rescue (gray bar). (F) Viral titers in the media from each condition were determined by viral plaque assay of 4 individual experiments. Values are mean and SD. ${ }^{*} p<0.05,{ }^{* *} p<0.01,{ }^{* * *} p<0.001,{ }^{* * *} p<0.0001$. 
A
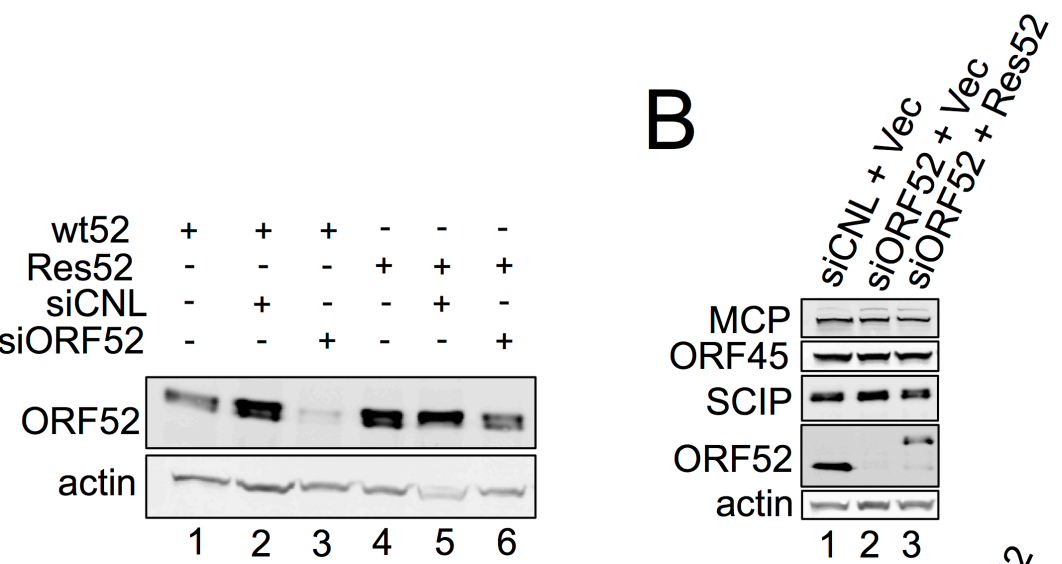

C

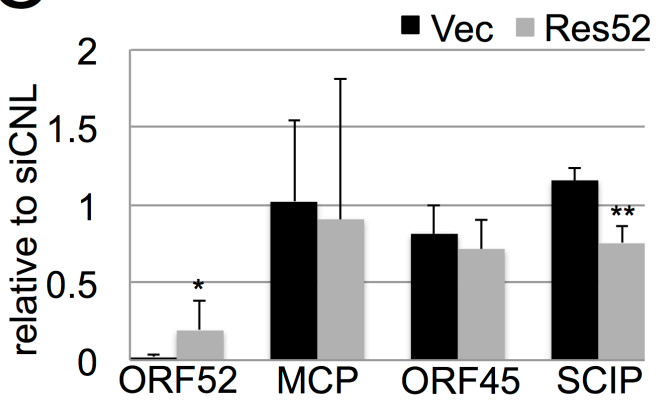

D
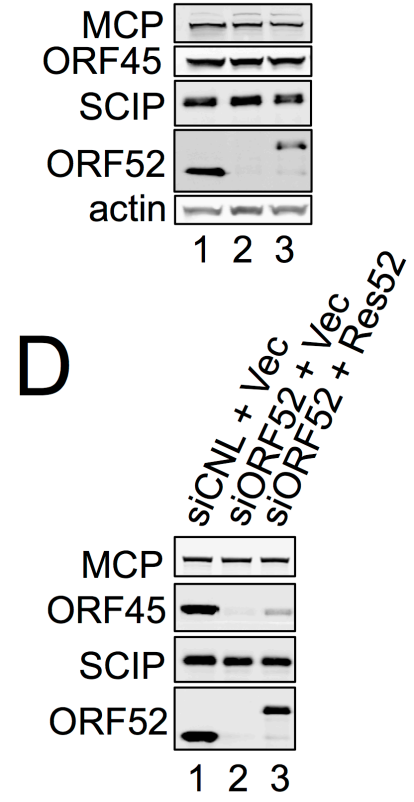

E

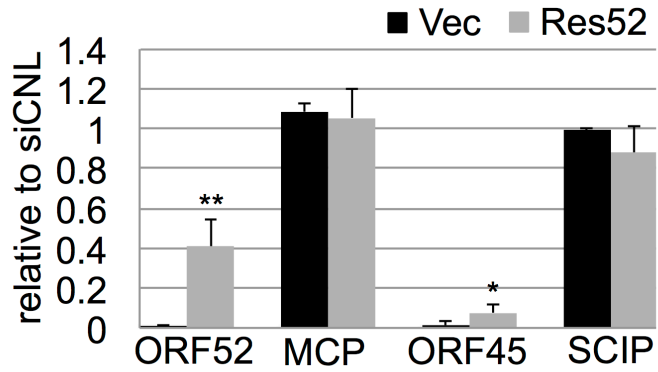

F

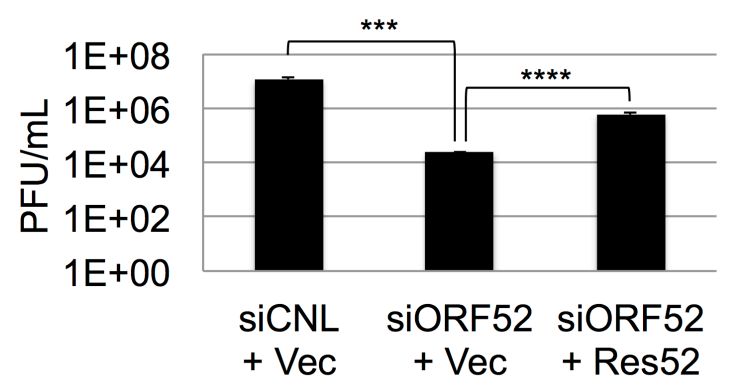


siORF52 showed a significant reduction in ORF52 (Figure 2-7B, lane 2), similar to the pattern we discerned in infected cultures pretreated with only siORF52 (Figure 2-1A and 2-1B). Aside from the near absence of ORF52 in cell lysates, production of other viral proteins that we examined was not significantly different from infected cells transfected with empty vector and siCNL (Figure 27C, black bars). In cell lysates from the complementing RhF cells (i.e. transfected with pk-myc-Res52, abbreviated as Res52 from this point onwards), we detected Res52 that, as we expected, migrated more slowly than wild-type ORF52 due to the myc tag (Figure 2-7B, lane 3). With the exception of SCIP that is modestly depressed (by approximately $25 \%$ ) in the $\mathrm{kd} / \mathrm{Res} 52$ condition compared to the control (kd/vector), the intracellular levels of the non-targeted structural proteins are similar in the two conditions (Figure 2-7C, gray bars). Expression of the transfected Res52 in siORF52 treated, RRV infected cells is notably lower than that of wt ORF52 encoded by the virus in cells treated with siCNL + vector, yet it is significantly higher (11-fold) than the wt ORF52 levels following knockdown alone (siORF52 + pk-myc vector) (Figure 2-7C)

Since Res52 was present in the rescue cell lysate (Figure 2-7B, lane 3), we hypothesized that if it were expressed at the correct time in the viral life cycle and in a useable form, we would observe some rescue of the knock down phenotype. We first examined particle composition from media collected from the control, knockdown, and rescue conditions. As we had observed previously with infected cells pre-treated with siCNL or siORF52 (Figure 2-3), the levels of particle-associated capsid proteins (MCP and SCIP) were similar in the presence 
or absence of complementing Res52 following ORF52 kd compared to controls (Figure 2-7D), and the two tegument proteins (ORFs 45 and 52) were absent following $\mathrm{kd}$ without ORF52 complementation (Figure 2-7D, lane 2). However, in the particles released into the media from the rescue condition, we now observed Res52 at a significantly increased level (40-fold) over the amount in without rescue (Figure 2-7D, lane 3 vs. lane 2). The presence of Res52 in these released particles indicated that the Res52 that we provided in trans was packaged within the virion. Of note, we also observed partial rescue (albeit proportionally less than for Res52) of ORF45 incorporation into particles (Figure 2-7, lane 3). The average of five experiments demonstrated an approximately 7 fold greater amount of ORF45 per particle (using MCP as a way to normalize for particle number) over the particles released from infected cells pre-treated with siORF52 without rescue (+ pk-myc vector). This suggested that ORF45 required the presence of ORF52 on particles for its stable incorporation within the virion (Figure 2-7D and Figure 2-7E) but also that exogenous expression of Res52 may not be optimal in allowing efficient production of fully wt virions.

We next tested whether the released particles that contained Res52 and ORF45 represented infectious virions that would, in turn, partially restore the suppressed viral titer. To address this, we assessed the viral titer of the media from the rescue vs. control conditions (i.e. ORF52 kd with or without Res52 expression in trans, respectively). siORF52 + pk-myc vector showed a decrease in titer of $>400$ fold compared to siCNL + pk-myc vector. However, the presence of Res52 with siORF52 was able to partially reverse this suppression, increasing 
the titer approximately 24 fold over siORF52 + pk-myc vector (Figure 2-7F).

Combined, these data argued that reduction of ORF52 was responsible for the significant decrease in viral titer and that this decrease was likely due to a lack of formation and release of mature virions. However, to demonstrate the latter more definitively, we next examined whether Res52 could also rescue the siORF52 block in particle maturation that was evident in our earlier TEM (Figure 2-6).

\section{ORF52 in trans partially rescued virion maturation}

To address more directly whether the increase in titer after Res52 expression in trans reflected an increase in the production of mature particles and, specifically, tegumentation and envelopment, we next asked whether we could discern an increase in the production and release of trilaminar virions. We again used thinsection TEM to examine the various stages of RRV maturation in cells, this time comparing those transfected with pk-myc or rescued with Res52, both in the presence of siORF52. Since our results indicated that particles packaged exogenously expressed Res52 that resulted in an increase in the production of infectious virions (Figure 2-7F), we also expected to observe a partial rescue in normal virion morphogenesis. Not surprisingly, A, B, and C capsids were present in the nuclei of infected cells from all three conditions: control, knockdown, and rescue (Figures $2-8 \mathrm{~A}, 2-8 \mathrm{~B}$, and $2-8 \mathrm{C}$ respectively). Likewise, examination of the cytoplasm in siCNL + pk-myc vector treated cells revealed multiple mature virions with tegument and envelope within large vesicles (Figure 2-8D) as well as cell associated virions on or near the plasma membrane (Figure 2-8G). In cells 
transfected with siORF52 + pk-myc vector, however, we once again found untegumented and nonenveloped particles that often surrounded but did not appear to enter vesicles (Figure 2-8E). Furthermore, these cells lacking ORF52 and transfected only with vector displayed a lack of cell surface-associated virions (Figure $2-8 \mathrm{H}$ ). In marked contrast, cultures under rescue conditions (siORF52 + Res52) displayed low but consistent levels of tegumented and enveloped virions located within cytoplasmic vesicles (Figure 2-8F) as well as cell surface-associated virions along the outer surface of the cellular plasma membrane (Figure 2-8I), reminiscent of the control phenotype. Consistent with the somewhat weak restoration of total intracellular ORF52, the degree of this rescued phenotype was less frequent than in the control cells.

Likewise, with levels of Res52 and ORF45 in released particles in the rescue conditions lower than ORF52 and ORF45, respectively, in control conditions (Figure 2-7E), we predicted that we would continue to find immature capsid-like particles in the media along with the recovery of at least some mature trilaminar virions. TEMs from the concentrated media of the rescue and control cells bore out these predictions. Cells treated with siORF52 but expressing Res52 still produced naked capsids but we also observed mature-appearing virions with evidence of tegument, envelope, and glycoproteins that resembled wt virions from infected cells treated with siCNL and pk-myc (compare Figure 2-8L with 2-8J). Combined, these TEM images provided additional evidence that ORF52 is necessary for RRV tegumentation and envelopment as well as subsequent release of free and cell-surface associated mature viral particles. 
Figure 2-8. Exogenous ORF52 rescues formation and release of tegumented and enveloped virions. RhF cells transfected with either empty myc-tagged vector $(\mathrm{Vec})$ or siORF52 resistant myc-tagged ORF52 (Res52) were transfected 24 hours later with siCNL or siORF52 and then, after an additional 24 hours, were infected at an $\mathrm{MOI}$ of $5.48 \mathrm{~h}$ p.i. later cells were fixed and examined by TEM at $40 \mathrm{~K}$ magnification. The nucleus of siCNL + vec (panel A), siORF52 + vec (panel B), siORF52 + Res52 (panel C) all contained A (empty arrowhead), B scaffold-containing (chevron), and C (arrow) capsids. The cytoplasm of siCNL+ Vec cells (panel D) contained large vesicles (ves) filled with multiple tegumented and enveloped virions; the cytoplasm of siORF52 + Vec (panel E) contained untegumented particles, many of which surrounded but were not within vesicles. siORF52 + Res52 cells (panel F) showed virions within cytoplasmic vesicles. In cells treated with siORF52 + Vec (panel H) cell associated virions are absent, in contrast to cells treated with siCNL + vec (panel G) or siORF52 + Res52 (panel I). Cells treated with siORF52 + Vec release particles that appear to be capsids, lacking tegument and envelope (panel $\mathrm{K}$ ), in contrast to cells treated with siCNL + Vec (panel J) and siORF52 + Res52 (panel L) that release intact, mature virions. Inset images are expanded $2 X$ from original images. 


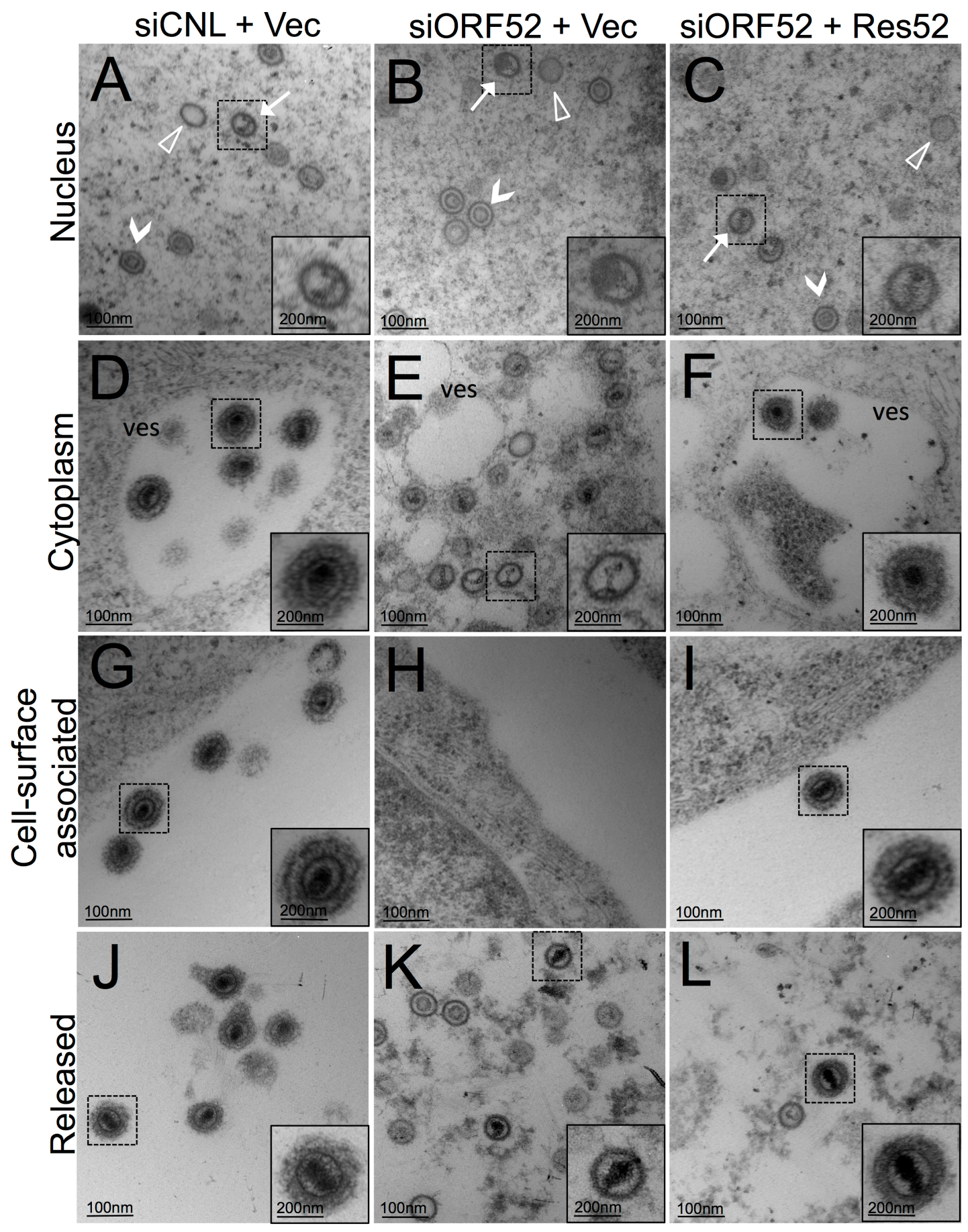




\section{Res52 partially restored nuclear egress of ORF45 in siORF52 treated cells}

Since providing Res52 in trans, in the presence of siORF52, partially rescued ORF45 incorporation into viral particles and led to an increase in the production and release of infectious virions, we also predicted that IF images would reveal a partial return to wild-type subcellular distribution of ORF45. To verify this, we again transfected RhF cells with vector or Res52 prior to ORF52 knockdown and RRV infection. 48 hours later pk-myc vector plus siCNL, as we had predicted, resulted in the normal cytoplasmic and nuclear distribution of ORF45 and only cytoplasmic localization for ORF52 (Figure 2-9, upper row). In siORF52 treated cells, the ORF45 expression again was only nuclear (Figure 2-9 middle row). However, with Res52 some ORF45 signal was again evident in the cytoplasm and appeared as punctate dots that co-localized with ORF52, suggesting that ORF45 and ORF52 were again packaged within virions (Figure 2-9, lower row, white arrows).

\section{DISCUSSION}

While there are a number of proteins (or their homologs) present in the tegument of all herpesvirus species (reviewed in (119)), others are restricted to a specific subfamily. These latter proteins likely have specialized roles critical in aspects of the life cycle or intracellular environment that are specific to those subfamilies. Most models of herpesvirus maturation suggest that final tegumentation begins once a capsid enters the cytoplasm following nuclear egress (reviewed in 
Figure 2-9. Complementation with siRNA-resistant ORF52 partially restored cytoplasmic subcellular localization of ORF45. RhF cells transfected with either empty myc-tagged vector (Vec) or siORF52 resistant myc-tagged ORF52 (Res52) were reverse transfected 24 hours later on coverslips with siCNL (upper panel) or siORF52 (middle and lower panels) and then, after 24 hours, infected with RRV at $\mathrm{MOI}$ of 5 and fixed 48 hours later. Cells were stained with antiORF52 and anti-ORF45 antibodies, followed by secondary antibodies Alexa Fluor 488 goat anti-mouse and Alexa Fluor 555 goat anti-rabbit, respectively. Cells were then stained with DAPI. Arrows in merge panels (upper and lower) highlight overlapping signals and colocalization of ORF52 and ORF45 in the cytoplasm. 


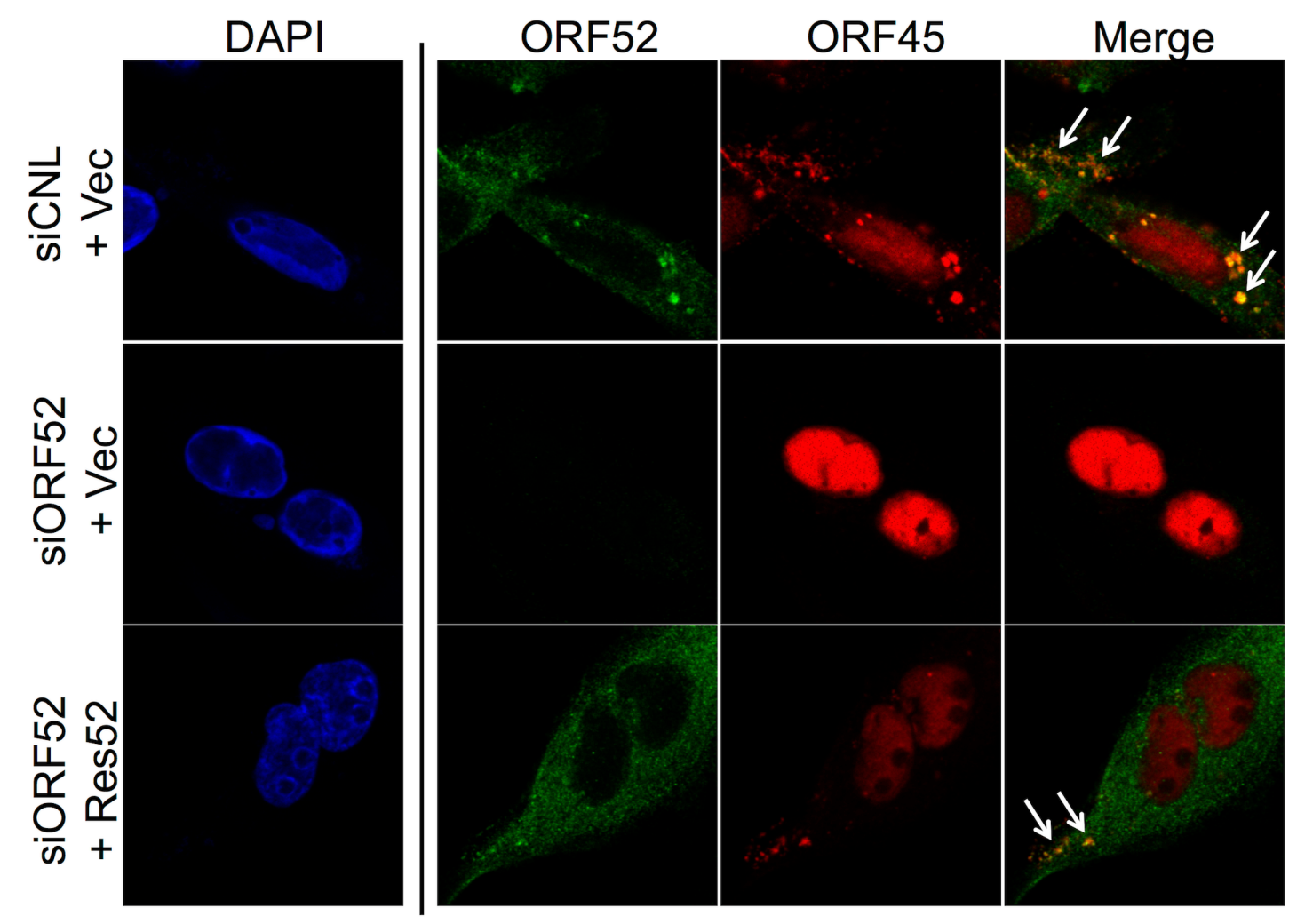


$(119,213))$. Capsids in the cytoplasm may then obtain a number of tegument proteins while moving toward vesicles, which may have an additional reservoir of tegument proteins (reviewed in $(119,211))$. These partially tegumented capsids reach the vesicles and then bud into them, acquiring additional tegument proteins as well as the envelope studded with glycoproteins already embedded in the vesicle membrane (reviewed in $(119,126))$. If tegumentation depends on the coordinated interaction of proteins to assemble, it follows that a lack of or defect in one of these proteins might disturb this process. Similarly, disruption in tegument assembly could also lead to particles lacking the proteins necessary to initiate and ensure receptor-mediated invagination, thereby preventing the acquisition of an envelope and inhibiting vesicle-mediated egress.

ORF52 is a tegument protein found only within the gammaherpesvirus subfamily. While previous studies have investigated both the structure and function of the murine homolog, MHV-68 ORF52 $(24,27,330)$, and most recently the importance of phosphorylation in the ability of BLRF2, the EBV homolog, to complement a null mutant of MHV-68 ORF52 (82), this is the first study investigating the role of ORF52 in RRV, a close homolog of the human pathogen, KSHV. RRV ORF52 is a tegument protein that is highly abundant within virions and tightly associated with the capsid (239). In this report, we show that ORF52 is necessary for tegumentation and secondary envelopment but is not essential for the production of structural proteins, assembly of capsids, viral DNA replication or packaging, or the ability of capsids to egress from the nucleus. Without ORF52, untegumented particles, which are morphologically 
indistinguishable from intranuclear capsids, accumulate within the cytoplasm (Figure 2-6), unable to enter but often surrounding cytoplasmic vesicles where they would otherwise undergo secondary envelopment (reviewed in $(126,213)$ ). These results are consistent with the observations from disruption of MHV-68 ORF52, which also leads to a defect in tegumentation and secondary envelopment $(27,330)$.

\section{Increased proportion of immature particles in the absence of ORF52}

Despite the inability of the untegumented and unenveloped particles to enter vesicles, we detected their release into the media at levels that were similar to those from control cells (Figure 2-3). At first this seemed contradictory in light of the approximately 4-fold greater cytoplasmic accumulation of particles in the ORF52 kd compared to controls, which we suggest reflects the inability of the untegumented particles to enter the vesicle-mediated egress pathway. However, we also noted that essentially no virions were associated with the cell surface in the $\mathrm{kd}$ conditions, whereas the controls displayed large numbers of virions that remained attached to the plasma membrane following egress (Figure 2-6E and Table 2-1), a typical phenomenon of herpesviruses, including $\operatorname{KSHV}(112,114)$. Thus, although the number of released particles in the media is similar, with or without ORF52 knockdown (Figure 2-3), the contribution of the cell surfaceassociated fraction argues that the number of virions exiting the cells, in total, is greater in the control than in the knockdown condition. Although the mechanism of release of the immature particles from the siORF52 treated cells remains 
unclear, we did investigate whether it was simply due to cell lysis. However, we found that even if we harvested media at $24 \mathrm{~h}$ p.i., a time in which infection induced lysis is negligible (Anderson and Kedes, unpublished observations), the number of particles in the media, though lower than at $48 \mathrm{~h}$ p.i., was similar between kd and control conditions (data not shown), arguing against this explanation. Furthermore, it is not immediately evident whether our results reflect a bona fide alternative egress pathway or arise as an artifact of the ORF52 kd conditions. Arguing against this last interpretation, however, is that even in control conditions, A, B and C capsids, together, comprised over $1 / 3$ of the total released particles. Nevertheless, the mechanism of vesicle-independent particle release, other than through cell lysis, remains unclear and is of continued interest to our laboratory.

\section{ORF52 is essential for virion maturation following egress from the nucleus}

Although ORF52 kd conditions led to an approximately 6-fold reduction in the proportion of DNA-containing particles in the media, we conclude that their lack of tegument and envelope accounted for most of the drastic loss in titer (>300fold) that we observed (Figure 2-1C) despite similar total concentration of particles between kd and control conditions (Figure 2-3). The released particles were morphologically indistinguishable from the accumulating cytoplasmic particles (and nuclear capsids), lacking any discernable evidence of tegument or envelope (Figure 2-5). Consistent with these observations, immunoblots demonstrated that these particles lacked tegument proteins ORF52 and ORF45 
(Figure 2-3). We also showed that these phenotypes are specific to ORF52, since myc-tagged ORF52 in trans partially rescued maturation with particle entry into vesicles and release of mature virions that was accompanied by the reappearance of cell-associated mature virions (Figure 2-7 and 2-8).

\section{ORF52 acts as a functional lynch pin for tegument formation}

Tegumentation is a complex process involving the layering of multiple interacting cellular and viral proteins, which occurs throughout the cytoplasm and at sites of secondary envelopment (reviewed in $(119,211,275)$ ). Earlier studies divide the tegument into "inner" and "outer" portions, referring to biochemical or structural evidence describing the relative affinity and proximity of specific sets of proteins to the surface of the capsid $(62,239,336,356,357)$. When one of these tegument proteins is missing, it affects the ability of other proteins to be added or perhaps remain stably attached to the particle. We postulate that this might explain the loss of tegumentation that we observed by TEM and, specifically, the defect in incorporation of tegument protein ORF45 during RRV maturation in the absence of ORF52.

In our work, we found that diminution of ORF52 critically affected the ability of particles to acquire this tegument layer post nuclear egress and these particles localized around but were unable to enter vesicles (Figure 2-6D). This result is consistent with data published on MHV-68 ORF52 where cells transfected with an ORF52-StopBAC, which expresses the entire viral genome, except ORF52, are also unable to undergo tegumentation and envelopment, as 
we have mentioned above (27). Recent work from the Deng laboratory demonstrated, by co-immunoprecipitation (co-IP) of transiently transfected HEK293 cells, that MHV-68 ORF52 interacts with another gammaherpesvirus specific tegument protein, ORF42, and that an arginine to alanine change at amino acid 95 (R95A) in ORF52 inhibited this interaction (330). The specific function of ORF42 is currently unknown; however, R95, which is conserved among ORF52 homologs in gammaherpesviruses, is important in the viral life cycle. The wt MHV-68 ORF52, but not the R95A mutant, complements the defect in replication of the ORF52-StopBAC (24). Other evidence from both KSHV and MHV-68 studies demonstrate that their respective ORF52 proteins interact with ORF45 and ORF42, respectively $(267,330)$.

In our experiments with RRV, we were unable to detect a direct interaction between ORF52 and ORF45 by IP either from HEK-293 cells expressing tagged versions of the two proteins (data not shown) or from RRV infected RhF. Since there are multiple proteins within the tegument layer interacting in yet unknown configurations, it is possible that ORF52 and ORF45 might indirectly interact through a third protein. A possible candidate is ORF33, a tegument protein present in alpha-, beta-, and gamma-herpesviruses. In MHV-68, ORF33 plays a role in virion maturation affecting both egress of capsids from the nucleus and tegumentation and envelopment of capsids reaching the cytoplasm (120). Particles released via freeze-thaw of cells transfected with a MHV-68 ORF33StopBAC have ORF52, but they lack ORF45 (120), suggesting that ORF33 might 
be a bridge between inner (e.g. ORF52) and outer (e.g. ORF45) tegument proteins.

\section{Nuclear egress of ORF45 is ORF52 dependent}

ORF45, a multifunctional protein with roles that include antagonizing the host antiviral response (360), is also a gammaherpesvirus specific tegument protein with homologs in RRV, KSHV, EBV, and MHV-68 $(23,28,142,239,358)$. KSHV ORF45 also interacts with a kinesin-2 motor protein, KIF3A, and is responsible for loading of viral particles onto microtubules for plus-end directed transport toward the plasma membrane (277). In our experiments, knockdown of ORF52 had no discernable effect on the overall ORF45 expression levels (Figure 2-1) yet it was not incorporated into released particles (Figure 2-3). This suggests that ORF52 is necessary for ORF45 incorporation (or at least its stable incorporation) into maturing virions and likely reflects the role ORF52 might have in establishing a tegument foundation that allows the stable addition of other tegument proteins rather than a direct interaction with ORF45 itself. In light of these findings, we hypothesized that if ORF45 were responsible for efficient particle transport, then knocking down ORF52 would affect tegumentation of particles in the cytoplasm and result in reduced movement through the cytoplasm and viral egress.

Consistent with a potential defect in anterograde transport, suppression of RRV ORF52 expression also appears to affect the subcellular localization of ORF45. During unperturbed RRV infection we found that in addition to a diffuse nuclear pattern, ORF45 also co-localized with ORF52 in the cytoplasm in a 
discrete punctate pattern. Following ORF52 kd, however, we detected ORF45 only in the nucleus. While we have no evidence that ORF52 can enter the nucleus (e.g. no NLS, no nuclear staining by IF) or interact directly with ORF45, these data argue that an intact tegument, dependent on ORF52 expression, is necessary for ORF45 incorporation into viral particles undergoing transcytosis. Capsid (SCIP-containing) particles from ORF52 kd lacked ORF45 and ORF52 in the cytoplasm (Figure 2-3 and 2-4) and demonstrated a more restricted and aggregated perinuclear distribution. This might reflect an inability to attach to microtubule-based motors (277).

\section{Inability of particles to invaginate into cytoplasmic vesicles}

During secondary envelopment in the cytoplasm, partially tegumented particles enter vesicles, acquiring their final complement of tegument proteins and their glycoprotein-studded envelope. In cells lacking ORF52, there was an accumulation of particles with no detectable tegument within the cytoplasm (Figure 2-6D). We found that vesicles in siCNL cells were larger than in siORF52 and many contained multiple trilaminar virions (Figure $2-6 C$ and $2-6 D$ ). Interestingly, cells that we treated with siORF52 still formed vesicles but the particles failed to enter and were often clustered around them (Figure 2-6D and 2-8E), consistent with ORF52null MHV-68 studies $(27,330)$. We speculate that this last step of invagination is dependent on proper tegumentation that provides key protein interactions between the surface of the particle and the surface of the vesicle. 
ORF52 dependent tegumentation and envelopment are necessary for cell-surface association of viral particles

Virions undergo release from the cell in a process similar to exocytosis once the vesicle containing virions merges with the cellular plasma membrane (reviewed in $(119,212,213))$. This leads to an opening of the vesicle where particles are released into the surrounding environment or remain associated with the cell surface. Part of the host immune function is to prevent the spread of infectious virions to other cells. Some virally infected cells, in fact, trap newly released enveloped virions, including retroviral and filoviral particles (146) and KSHV (192), onto the outer surface of the plasma membrane using bone marrow stromal cell antigen 2 or BST2/tetherin. BTS2, a heavily glycosylated type II transmembrane cellular protein (172), likely acts by forming homodimers that link the viral envelope and plasma membrane (reviewed in (88)). KSHV partially circumvents this inhibition by encoding K5/MIR2 that ubiquinates BST2, leading to its degradation (192). RRV, interestingly, has no K5 homolog. We observed mature RRV particles lining up along the cell surface during RRV infection in control but not in ORF52 kd conditions (Figure 6F), suggesting that without the envelope and its glycoproteins, no tethering of particles occurs.

\section{ORF52 in trans partially rescues block in particle maturation}

To ensure that ORF52 was responsible for the phenotypes that we observed, we complemented the system by providing siRNA resistant ORF52 (Res52) in trans. Expression of Res52 (Figure 2-7B and 2-7C) during siORF52 treatment of 
infected cells led to the incorporation of Res52 and, to a lesser extent, ORF45 into virions (Figure 2-7D and Figure 2-7E), suggesting that at least tegumentation was partially restored. This complementation also had functional consequences, increasing the viral titer compared to ORF52 knockdown alone (Figure 2-7F), and suggesting a partial restoration of fully infectious trilaminar virions. TEM images from Res52 complemented infections corroborated this hypothesis, demonstrating evidence of mature virions within vesicles (Figure 2$8 \mathrm{~F})$, cell-associated virions that appeared identical to those without knockdown (Figure 2-8G and 2-8I) and released virions also with visible tegument, envelope, and glycoproteins (Figure 2-8L). The efficiency of the rescue was likely suboptimal due to low plasmid transfection efficiency $(\sim 30 \%)$ of Res52 in the culture and the possibility that ectopic over expression likely fails to recapitulate the coordinated expression of interacting tegument proteins.

With MHV-68, the expression in trans of its own ORF52, or the homolog from KSHV or EBV (BLRF2), is able to complement a similar defect in virion maturation and total block in virion release that arises with transfection of cultures with an ORF52-stop BAC (330). We also attempted to complement the ORF52 $\mathrm{kd}$ induced defect in tegumentation in the RRV system with KSHV ORF52 but found that despite its expression being comparable to that of RRV Res52, its incorporation into the virions was poor and, as a consequence, the viral titer increased by only 1.5 fold (compared to 26 -fold increase with RRV Res52) over ORF52 knockdown controls (data not shown). The reasons behind the differences between the MHV-68 and our RRV results are unclear. 
In sum, we have shown that the inner tegument protein RRV ORF52 is critical for post-nuclear stages of virion maturation, including tegumentation and secondary envelopment necessary for production and egress of fully mature trilaminar virions. Without ORF52, the necessary layering of tegument proteins key to the architecture of the virion - is unable to occur, resulting in the cytoplasmic accumulation of immature viral particles surrounding but unable to enter awaiting vesicles. 
CHAPTER 3: RRV ORF52 COLOCALIZES WITH MICROTUBULES AND INDUCES A BUNDLING PHENOTYPE 
Abstract

We have found that, when over-expressed, the gammaherpesvirus specific tegument protein RRV ORF52, co-localizes with microtubules and can lead to polyploidy as well as microtubule bundling. In addition, RRV ORF52 appears to associate with microtubules in pull down experiments when cytoplasmic extracts are present but only minimally in a purified system, suggesting the role of a cofactor or microtubule-binding partner in mediating the phenotype. We propose that ORF52 plays a role not only in the structure of virions but also in viral particle assembly as well as transcytosis during egress and/or entry via a direct or indirect interaction with microtubule components of the cytoskeleton.

\section{Introduction}

Microtubules (MTs), actin microfilaments, and intermediate filaments (IFs) make up the eukaryotic cytoskeleton providing various functions including maintenance of cell shape $(99,201)$, cell motility $(15,19)$, and movement of chromosomes during mitosis and cargo throughout the cell $(111,175,190)$. Following herpesvirus envelope fusion and release of the unenveloped capsid into the cytoplasm, the majority of tegument proteins are lost from the particle and released into the cell. In HSV-1 two viral tegument proteins remain associated with the capsid following entry, VP1/2 (encoded by UL36) and UL37 (113, 187, 195). While neither VP1/2 nor UL37 interact with motor proteins, both are thought to somehow recruit dynein (256). 
Cargo transported throughout the cell also includes viruses that utilize the host cytoskeleton, specifically MTs, during entry, movement through the nucleus and cytoplasm, and egress (reviewed in $(19,67,75,155,188,209,212$, $213,251,308,352))$. Viruses, including herpesviruses, rely on the MT network for transportation throughout the cell during entry, egress, and additional stages during the viral life cycle $(34,74,116,198,225,255,261,282,295,300,335)$.

\section{Microtubules}

MTs are made up of $\alpha$ and $\beta$ tubulin subunits that join together in the presence of $\mathrm{Mg}^{2+}$ and GTP in an end-to-end manner to form protofilaments, giving microtubules an intrinsic polarity. 13 protofilaments then associate to form a hollow $25 \mathrm{~nm}$ microtubule (Figure 3-1) (8). Due to their polarity, MTs have a designated plus and minus end. $\alpha$ - and $\beta$ tubulin heterodimers can add or dissociate at either end of a MT; however, there is greater tendency for addition to occur at the faster growing plus end where $\beta$-tubulin is exposed and depolymerization to occur from the minus-end. Both $\alpha$ and $\beta$ tubulins exist in several isotypic forms and can undergo several post-translational modifications. Drugs and temperature can also alter MT dynamics. For example, nocodazole and colchicine in addition to cold temperatures block the polymerization of tubulin into microtubules, whereas the taxane drug class (e.g. paclitaxel or docetaxel), stabilize GDP-bound tubulin in the microtubule $(186,266)$. 
Figure 3-1. The structure of a microtubule and its subunit. (A) The subunit of each protofilament is a tubulin heterodimer, formed from a very tightly linked pair of $\alpha$ - and $\beta$-tubulin monomers. (B) One tubulin subunit ( $\alpha-\beta$ heterodimer) and one protofilament. Each protofilament consists of many adjacent subunits with the same orientation. (C) The microtubule is a stiff hollow tube formed from 13 protofilaments aligned in parallel. Image credit: Mary A. Maxfield and used with permission. 


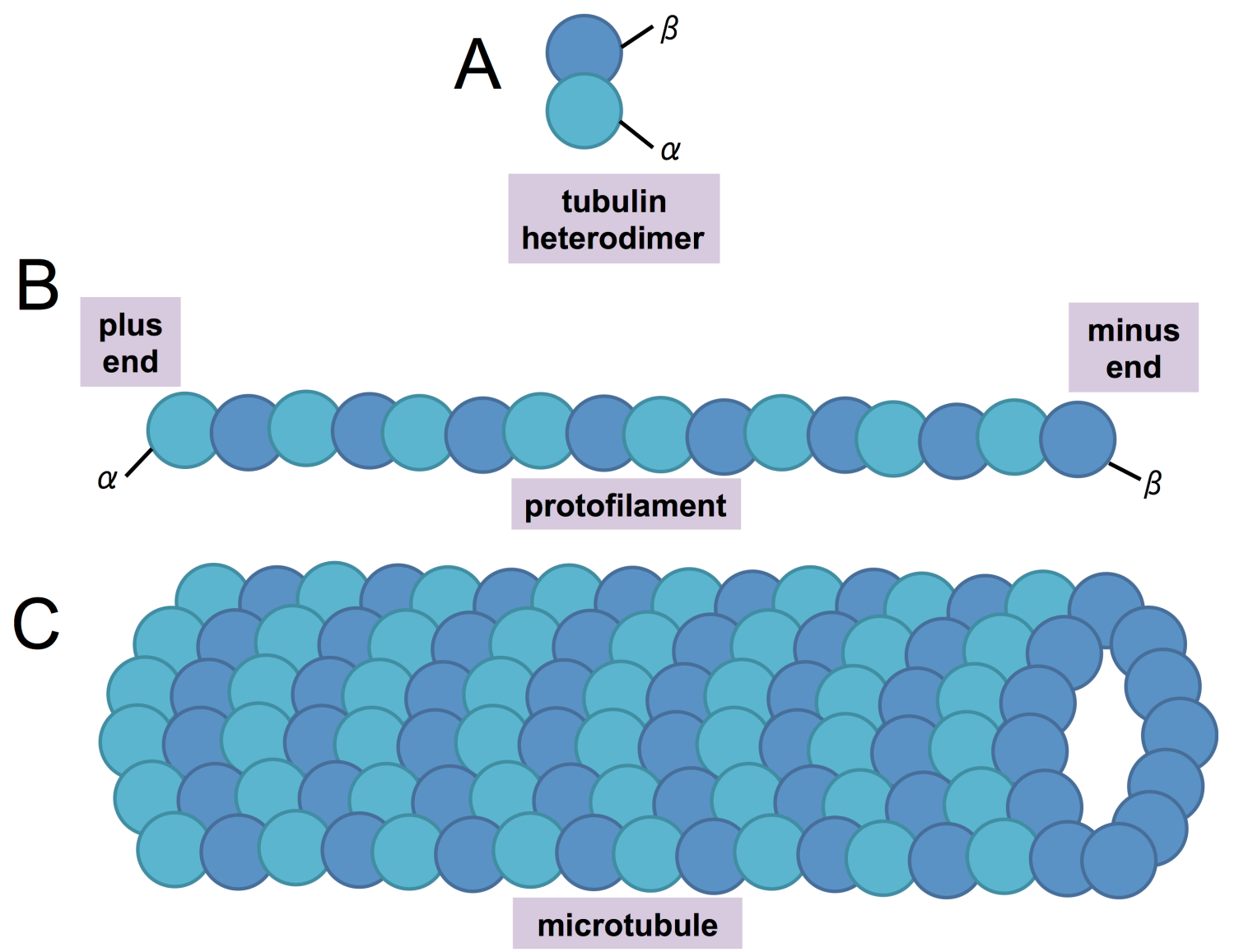




\section{Microtubule associated proteins}

Structural microtubule associated proteins (MAPs) bind reversibly with lowmolecular range affinities to MTs (194). Structural MAPs, which include the neuronal proteins MAP1, MAP2 and tau as well as MAP4, which is present in all non-neuronal cells, promote tubulin polymerization and stabilize MTs, often in the form of "bundles" that look like thick cords of MTs $(80,314)$. MAP2, tau and MAP4 are made up of an N-terminal domain protruding from the MT surface and a C-terminal MT binding domain containing a proline-rich sequence and three or four 31 residue pseudorepeats (reviewed in (81)). The MT-stabilizing capacity of MAPs is greatly influenced by phosphorylation $(25,80,314)$. Growth factor signals can activate protein kinases that catalyze phosphorylation of tubulinbinding domains of MAPs and allow them to detach from MTs. Phosphorylation of MAPs is shown to decrease their binding capacity for MTs, destabilizing the MTs (136). The HSV-1 tegument protein VP22 colocalizes with MTs inducing stable bundles reminiscent of cellular MAPs suggesting it might be the first recognized virally encoded MAP (86). Additionally, VP22 interacts with gD, which also interacts with the viral capsid (56).

\section{Motor proteins}

Motor proteins are a type of MAP. Current knowledge of motor proteins in mouse and humans identifies 45 kinesin motors, 15 dynein and 40 myosin genes, categorized into 5 kinesin, 3 dynein, and 2 myosin subclasses based on sequence and functional motor comparison (reviewed in $(74,130,321,326)$ ) 
(Figure 3-2, myosin not shown). Kinesins bind microtubules and hydrolyze ATP to produce movement toward microtubule plus ends, typically extending out toward the cell periphery. These motor proteins are made up of an $\mathrm{N}$-terminal, ATP-hydrolyzing domain, a central coiled stalk region and a C-terminal tail region, which is most often the site of cargo binding (reviewed in (189)). Kinesin2 is a MT-based plus-end-directed motor protein, moving cargoes from the nucleus out toward the cell periphery $(129,342,344)$. Dyneins are also microtubule motors; however, they move cargoes toward the minus end of the microtubules, (reviewed in $(320,321))$ (Figure 3-3). The dynein motor domain is made up of a hexamer of AAA domains (ATPases Associated with cell Activities), which bind and release proteins in an ATP-dependent manner (reviewed in (320, 321)). Dynein is associated with additional proteins including light intermediate chains, intermediate chains and light chains ('chain' refers to a polypeptide subunit of the protein complex). Additionally, dynein associates with the multisubunit complex, dynactin, which stimulates dynein-cargo interaction and long distance movement of dynein (reviewed in $(320,321)$ ).

Many viruses, including herpesviruses, have found ways to utilize the cellular cytoskeleton, specifically MTs, for transportation and viral spread ((277) and reviewed in $(34,74,75,255,331)$ ); however, the mechanism (i.e. specific protein-protein interaction between cellular and viral proteins) is not clear. Studies from multiple groups have identified kinesin- 1 and cytoplasmic dynein as the two most frequently used motor proteins in viral transport (reviewed in (74)). However, in KSHV, the tegument protein ORF45 docked the entire KSHV viral 
Figure 3-2. Structure of common motor proteins. (A) Cytoplasmic dynein heavy chains (DHC; purple) comprise the globular heads and a stalk that ends in a MT-binding domain. The N-terminal DHC stem binds dynein light intermediate chains and dynein intermediate chains (blue). (B) Dynactin consists of a rod of Arp1 protein, and a flexible sidearm of the protein p150 Glued. Dynein is thought to bind to dynactin along or near the base of the sidearm. (C) Kinesin-1 has a rodlike structure with two heads, a stalk, and a splayed end. Kinesin-1 is a heterotetramer of two kinesin heavy chains (purple) and two kinesin light chains (blue). (D) Kinesin-2 is a heterotrimer of two KIF3 chains (purple) that form globular heads, and one kinesin-associated protein (blue). (E) Kinesin-4 is a dimer of two KIF4 chains with two globular heads, a central $\alpha$-helical coiled-coil stalk and a globular tail. Image credit: Mary A. Maxfield and used with permission. 

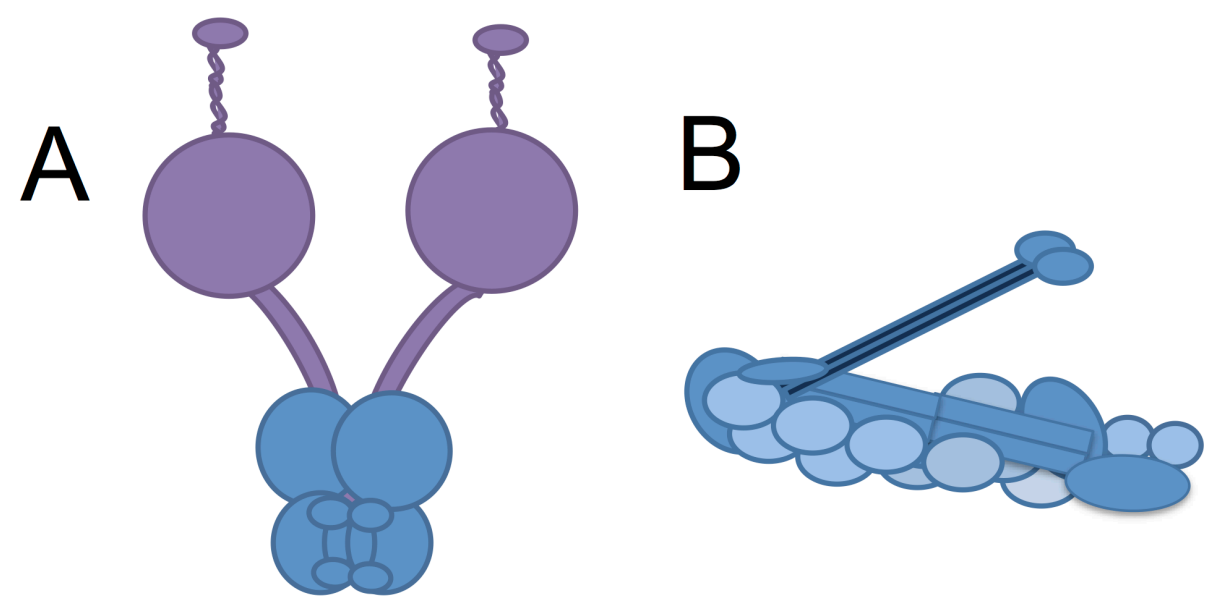

\section{Cytoplasmic} dynein

Dynactin

C

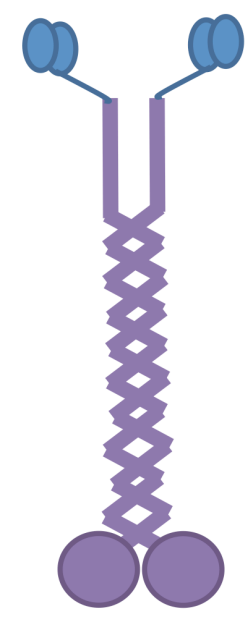

D

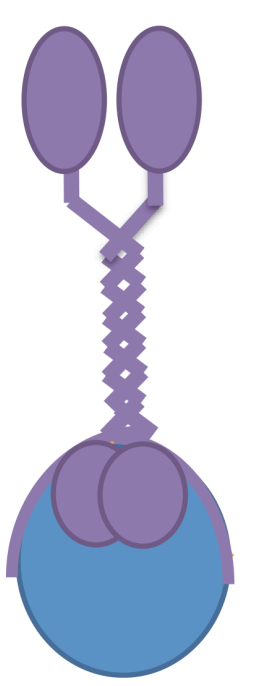

E

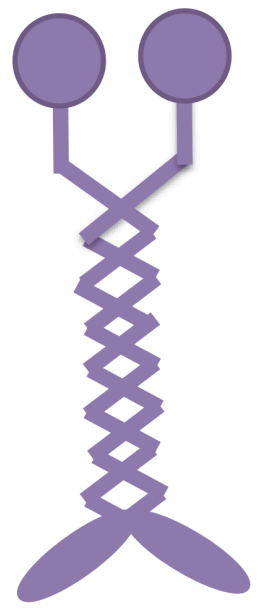

Kinesin-1

Kinesin-2

Kinesin-4 
Figure 3-3. Organization of microtubules in an unpoloraized fibroblast cell. Organization of microtubules (MTs) (red) in an unpolarized fibroblast cell, radiate from the MT-organizing center (MTOC) to the cell periphery, where their plusends $(+)$ are located. The MTOC nucleates MT minus-ends $(-)$ and is often close to the nucleus. Image credit: Mary A. Maxfield and used with permission. 


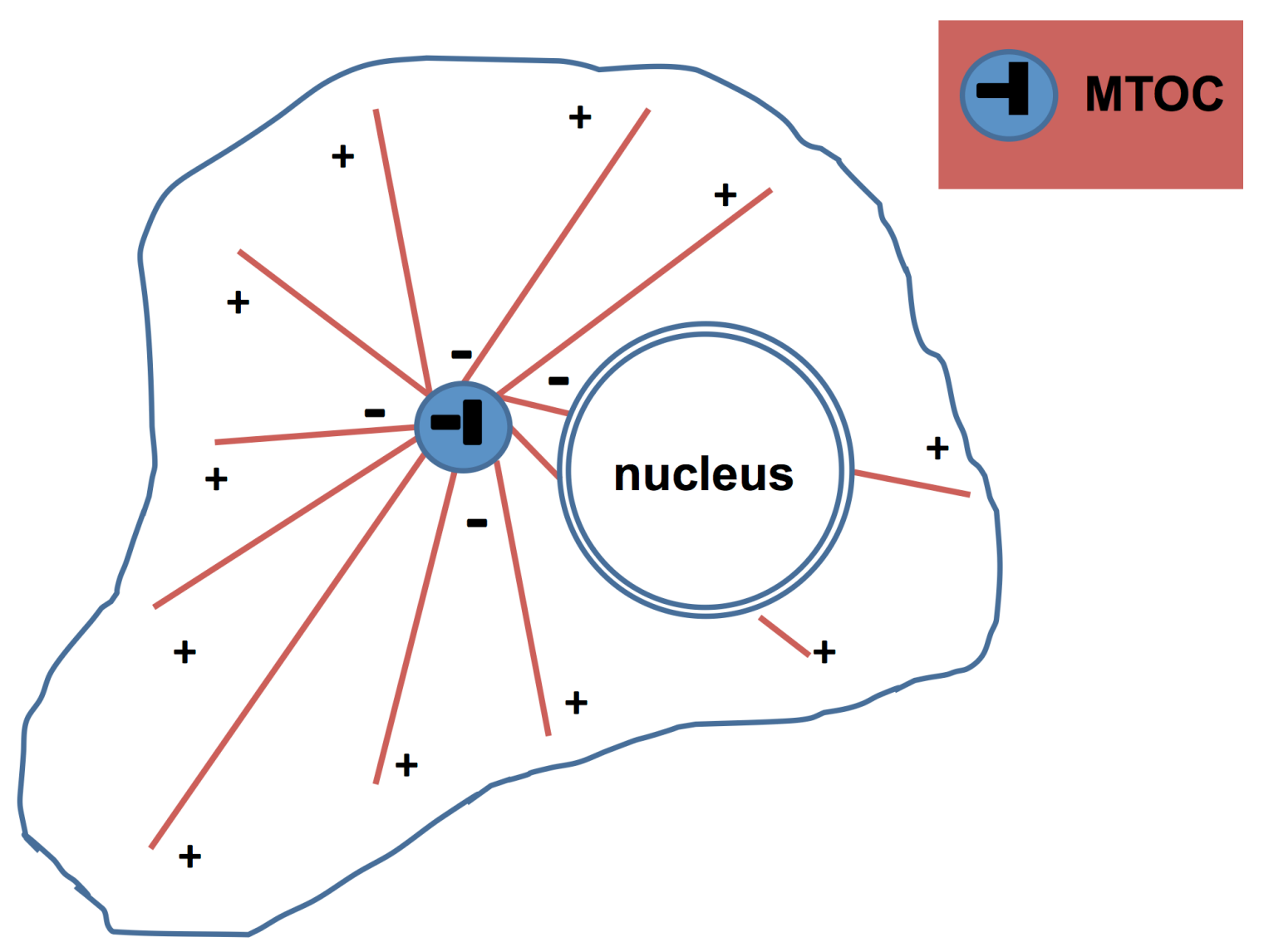


capsid-tegument complex onto KIF3A, a subunit of the kinesin-2 motor protein that was then translocated along MTs for maturation and egress (277). In our laboratory we have found that upon transient transfection of RRV ORF52 into HEK293, RhF, and HeLa cell lines, microtubules became thicker and adopted a bundle-like phenotype that was resistant to the depolymerization action of cold temperatures or nocodazole (Veriville and Kedes, unpublished data). This bundling was most pronounced when ORF52 was highly expressed such as in HeLa cells, but was also clearly evident in RhF cells leading us to question whether ORF52 might be a virally encoded MAP.

\section{Materials \& methods}

\section{Cell Culture}

Telomerase-immortalized rhesus monkey fibroblasts (RhF) were grown in complete media (Dulbecco's modified Eagle's medium) [Gibco] supplemented with $1 \mathrm{nM}$ puromycin, $1 \mathrm{mM}$ sodium pyruvate, and $10 \%$ fetal bovine serum [Gibco]). HEK293 and HeLa cells were grown in complete media (Dulbecco's modified Eagle's medium [Gibco] supplemented with $10 \%$ fetal bovine serum [Gibco]).

\section{RRV Stocks}

RhFs were grown to confluency, approximately $2 \times 10^{7}$ cells in a T182 flask, and infected with RRV strain $\mathrm{H} 26-95$ at a multiplicity of infection (MOI) of 0.05 in $5 \mathrm{~mL}$ complete media for 1 hour. Cells were then supplemented with an additional 100 
$\mathrm{mL}$ of complete media per flask. Supernatants were collected 5-7 days postinfection (p.i.) and cleared of cellular debris by low-speed centrifugation at $350 \mathrm{x}$ g. Cleared viral supernatants were passed through a $0.45 \mu \mathrm{m}$ pore size filter. Virus was concentrated by centrifugation for 3 hours at $12,855 \mathrm{xg}$ in a Sorvall SL250T rotor. Resulting viral pellets were resuspended in $1.0 \mathrm{~mL}$ TNE $(20 \mathrm{mM}$ Tris $[\mathrm{pH} 7.5], 100 \mathrm{mM} \mathrm{NaCl}, 1 \mathrm{mM}$ EDTA), titer was determined, and $20 \mu \mathrm{L}$ aliquots were stored at $-80^{\circ} \mathrm{C}$.

\section{ORF52 plasmid and generation of arginine to alanine mutant plasmid}

$\mathrm{N}$-terminal myc-tagged siORF52 resistant RRV ORF52 was generated from a pK-myc wt RRV ORF52 plasmid. The pK-myc plasmid was derived from pKH3 backbone and a gift from Deborah Lannigan and lan Macara. Full-length ORF52 sequence was amplified by PCR using purified RRV DNA as a template and primers that added a Notl and an EcoRI sequence to ORF52 for cloning (NotlORF52-F 5'-ATAAGAATGCGGCCGCTTATGTCTTCCACGCGT-3' and EcoR1ORF52-R 5'-GGAATTCCTAGTCCGCGTCGTTATT-3'). RRV ORF52 was cloned into the pK-myc vector at the Notl and EcoRI sites. The sequence of the wt pKmyc-RRV52 plasmid was confirmed prior to further use by sequencing with Sp6 primers (GeneWiz) and comparing with published wt ORF52 sequence. wt pKmyc RRV ORF52 plasmid was used as a template to generate an RRV ORF52 arginine to alanine mutant plasmid (R103A) 'R95A' is MHV-68 nomenclature, but the conserved arginine is 103 in RRV. Primers were designed that would alter one nucleotide, 
Fwd: 5‘- CCTCGCCGGAATTAGTATCGCCGTGGACGTGTCAATGGACG -3’

Rev: 5'- CGTCCATTGACACGTCCACGGCGATACTAATTCCGGCGAGG -3'

The R103A mutant plasmid was generated using the primers containing the desired mutations, the wt pK-myc ORF52 plasmid, and the QuikChange Lightening Site-Directed Mutagenesis Kit (Life Technologies). The mutations were verified by sequencing of the pk-myc-ORF52R103A plasmid with Sp6 fwd primer.

\section{Immunofluorescence assay (IFA)}

48 hours post-transfection cells were washed in PBS and fixed in $4 \%$ paraformaldehyde (PFA) for 10 minutes at room temperature. After two additional washes, the cells were permeabilized with $0.2 \%$ TritonX-100 in $10 \%$ normal goat serum (NGS) in PBS for 20 minutes. Then the cells were incubated with mouse anti-ORF52 and FITC-conjugated mouse anti-tubulin (Sigma) primary antibodies in $10 \%$ NGS in $0.05 \%$ Tween in PBS for 60 minutes at room temperature. After three additional washes, the cells were incubated with Cy3-goat-anti-mouse secondary antibody in $10 \%$ NGS in $0.05 \%$ Tween in PBS for 45 minutes at room temperature. Before the cover slip was sealed, the cells were treated with 0.5 $\mu \mathrm{g} / \mathrm{mL}$ DAPI (4',6-diamidino-2-phenylindole) to stain the nuclear DNA. Fluorescent images were photographed with a Nikon Eclipse TE2000E inverted microscope. 


\section{Transfection of ORF52}

For IF experiments, $2 \times 10^{6} \mathrm{RhF}$ cells were transfected via Amaxa nucleofector program T-016 with $2 \mu \mathrm{g}$ of pkmyc-empty vector, pkmyc-ORF52, or pkmycR103AORF52 plasmid and $3 \times 10^{4} \mathrm{RhF}$ cells were plated in an 8 well chamber slide (BD Biosciences). For transfection of RhF with ORF52 for MT pull down the same transfection conditions were used, but additional cells were plated in 10 $\mathrm{cm}^{2}$ plates. For transfection of HeLa and HEK293 cells, plasmid DNA was transfected with Lipofectamine 2000 (Life Technologies) into 90\% confluent cells plated in 8 well chamber slides (IF) or $10 \mathrm{~cm}^{2}$ plates (MT pull down) per manufacturer protocol.

\section{Microtubule spin-down assay}

\section{Polymerization of MTs for purified binding assay}

Microtubule Binding Protein Spin-down assay kit from Cytoskeleton.com. To 100 $\mu \mathrm{g}$ of tubulin protein in $20 \mu \mathrm{L}$ 1X BRB80 buffer [80 mM pipes, $1 \mathrm{mM} \mathrm{MgCl} 2,1$ mM EGTA] plus $2.5 \mu \mathrm{L} 100 \mathrm{mM}$ GTP add $2.5 \mu \mathrm{L}$ of cushion buffer (1X BRB80 buffer $+50 \%$ glycerol) and incubate at $35^{\circ} \mathrm{C}$ for 20 minutes to allow for polymerization of MTs. After 20 minutes we added $2 \mu \mathrm{L}$ of $2 \mathrm{mM}$ Taxol in DMSO to $200 \mu \mathrm{L} 1 \mathrm{X}$ BRB80 and combined BRB80 + Taxol with polymerized MTs and let sit at RT. MTs are between 5-10 $\mu \mathrm{m}$ in length and a concentration of approximately $5.0 \times 10^{11} \mathrm{MT} / \mathrm{ml}$, equivalent to $5 \mu \mathrm{M}$ tubulin dimer or $0.4 \mathrm{nM}$ MTs. 


\section{MT binding assay using purified MTs and ORF52}

Purified RRV ORF52 GST-fusion protein was made previously in our laboratory (Nancy Verville) in E. coli. Following purification and cleavage of GST, approximate concentration was determined by OD 280 reading.

1-3 $\mu \mathrm{g}$ of purified ORF52 protein in $1 \mathrm{X}$ BRB80 to final volume of $30 \mu \mathrm{L}$ was combined with $20 \mu \mathrm{l}$ polymerized MTs (see above) or $100 \mu \mathrm{g}$ of tubulin protein in $20 \mu \mathrm{L}$ 1X BRB80 buffer with no GTP (unpolymerized) and incubated at RT for 30 minutes. $50 \mu \mathrm{L}$ of protein and MT mixture was layered over $100 \mu \mathrm{L} 1 \mathrm{X}$ BRB80 + 50\% glycerol cushion in ultracentrifuge tubes (Hitachi Koki \#339133A). We spun samples at $100,000 x g$ in a Beckman ultracentrifuge (Todd Stukenberg laboratory) at $24^{\circ} \mathrm{C}$ for 35 minutes. Following centrifugation, $40 \mu \mathrm{L}$ was removed from the very top of the sample as "Supernatant", the remaining cushion was discarded, and the pellet was resuspended in $40 \mu \mathrm{L} 1 \mathrm{X}$ BRB80 buffer. $4 \mu \mathrm{l}$ of supernatant and pellet samples were resupsended in lithium dodecyl sulfate LDS sample buffer (NuPage; Life Technologies) with NuPage sample reducing agent (50 mM dithiothreitol (DTT)). Following denaturation by boiling for 10 minutes, proteins were separated by sodium dodecyl sulfate polyacrylamide gel electrophoresis (SDS-PAGE) on 12\% Bis-Tris gels (NuPage; Life Technologies).

For immunoblot analyses, proteins separated by SDS-PAGE were transferred to polyvinylidene fluoride (PVDF) membrane for 60 minutes at 250 $\mathrm{mA}$ at $4^{\circ} \mathrm{C}$. The membranes were blocked in $5 \%$ nonfat milk-TBS $(20 \mathrm{mM}$ Tris base, $150 \mathrm{mM} \mathrm{NaCl}, 3 \mathrm{mM}$ Tris- $\mathrm{HCl}$ ) for 90 minutes at room temperature and then incubated with the primary antibodies mouse anti-RRV ORF52 $(1: 5,000)$ 
and mouse anti-alpha tubulin clone DM1 (Sigma) (1:500) diluted in 5\% nonfat milk-TBS-Tween (TBST) $(0.05 \%)$ overnight at $4^{\circ} \mathrm{C}$. After three 10 minute washes with TBST at room temperature (RT), membranes were incubated with HRPgoat-anti-mouse (Jackson Immunological) secondary antibody for 45 minutes at RT. Following two 10 minute washes in TBST and 1 in TBS, blots were exposed with a 1:1 ratio of Western Lightning Plus enhanced luminol reagent (PerkinElmer) and exposed to film.

\section{MT pull-down assay in transfected cells}

HeLa and RhF cells were transfected with pkmyc-ORF52 or pk-mycVector (mock) for 48 hours. Cells were washed in 1X PBS and collected in 1X BRB80 buffer supplemented with $1 \mathrm{X}$ complete pill protease inhibitor (Roche) and 1 $\mu \mathrm{g} / \mathrm{mL}$ cytochalasin D. Cells were lysed by sonication in a an ice water bath 5 times for 3-5 seconds each time. Nuclei were pelleted by centrifugation at $12,000 \mathrm{rpm}$ for 30 minutes at $4^{\circ} \mathrm{C}$. Protein concentration was determined by OD280 reading and $\sim 400-900 \mu \mathrm{g}$ of protein was used for each MT spin down assay. For polymerized samples we added $0.5 \mathrm{mM}$ GTP and $5 \mu \mathrm{M}$ Taxol and allowing for RT incubation for 5 minutes. Subsequently an additional $15 \mu \mathrm{M}$ Taxol was added and incubated for 30 minutes at $37^{\circ} \mathrm{C}$. For unpolymerized samples, no GTP or Taxol were added at anytime. Samples were layered over a $40 \%$ glycerol cushion in 1X BRB80 and centrifuged at 40,000 rpm for 40 minutes, at $25^{\circ} \mathrm{C}$. Supernatant was removed, cushion was discarded, and loading buffer was added to pellet. Samples were boiled for 10 minutes and separated by sodium 
dodecyl sulfate polyacrylamide gel electrophoresis (SDS-PAGE) on 12\% Bis-

Tris gels (NuPage; Life Technologies) followed by immunoblot analysis (see above for protocol).

\section{Results and Discussion}

RRV ORF52 colocalizes with microtubules and induces a bundle-like microtubule phenotype

In our earliest attempts to characterize RRV ORF52, we sought to determine localization of ORF52 upon transient transfection. We transfected HEK293 cells (Figure 3-4A) or RhF cells (Figure 3-4B) with pkmyc-ORF52 and fixed cells 48 hours later for IF. ORF52 localized to the cytoplasm, but what we also noticed is that ORF52 often displayed a staining pattern reminiscent of tubulin. We found when staining for tubulin that in some transfected cells MTs had a thicker, bundle-like appearance (Figure 3-4, white arrows). Furthermore, the appearance of MT bundling was increased with greater amounts of ORF52 transfected into the cells. We tested between $11 \mathrm{ng}-1.5 \mu \mathrm{g}$ ORF52 plasmid in $1 \times 10^{6}$ cells, and while there was a thickening of MTs even at the lowest concentration, the number of cells expressing bundling, and the extent of bundling, was greatest at $1.5 \mu \mathrm{g}$ ORF52 plasmid (data not shown). We also performed a time course, staining cells transfected at the higher concentration of ORF52 at 12, 24, and 48 hours post-transfection. We saw minimal bundling at 12 hours, more at 24 hours, and the greatest amount at 48 hours post-transfection (data not shown). 
Figure 3-4. Ectopic expression of ORF52 in transfected cells; ORF52 colocalizes with MTs and induces a bundle-like MT phenotype. (A) HEK293 cells or (B) RhF cells transfected with pkmyc-ORF52 for 48 hours and stained with Dapi, anti-tubulin, and anti-ORF52 antibodies. Arrows highlight transfected cells with bundled MTs. 


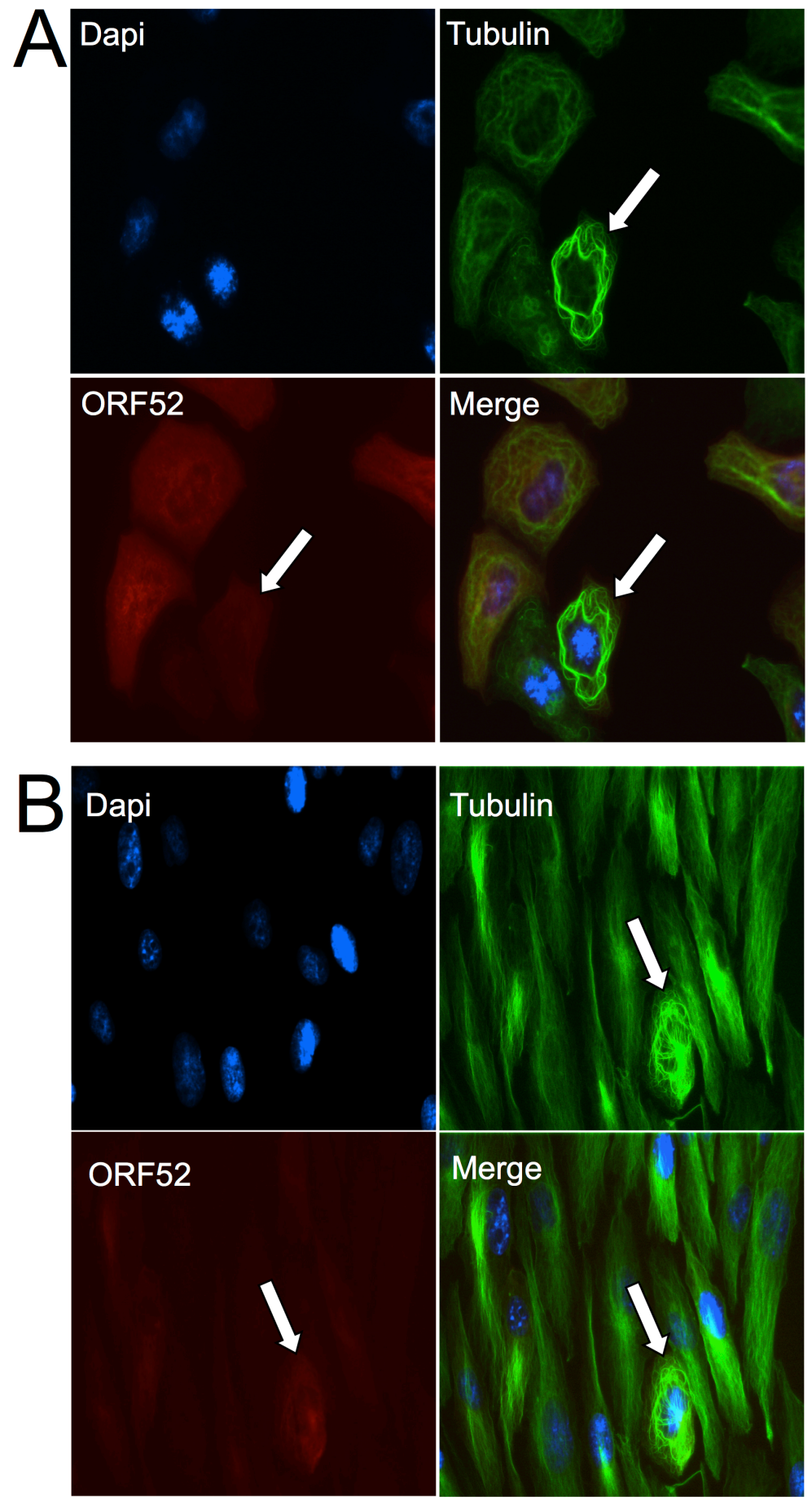


RRV infection also leads to an ORF52 staining pattern that resembles cytoskeletal MTs.

We also examined RhF cells during the course of wt RRV infection. RhF cells infected at an $\mathrm{MOI}$ of 5 for 48 hours and probed with anti-ORF52 showed a staining pattern in some cells suggestive of MTs (Figure 3-5), however tubulin was not co-stained so the MT-like pattern is only suggestive. While the MT bundling is much less during infection with RRV than transfection with ORF52, there is often colocalization between ORF52 and tubulin (data not shown). Our preliminary experiments with RRV indicate potential colocalization between ORF52 and tubulin and need to be further examined.

\section{Purified ORF52 interacts weakly with purified MTs}

In our IF experiment examining cells following transfection of ORF52, we noticed a colocalization between ORF52 and tubulin (Figure 3-4) as well as a thickening or bundling of MTs in many of the transfected cells (Figure 3-4, white arrows). The above observations led us to question whether ORF52 might be directly binding to MTs like other known cellular MAPs. To examine this potential interaction, we utilized a microtubule pull-down assay where we combined polymerized microtubules with purified RRV ORF52 protein and then, following centrifugation, analyzed both the supernatant and pellets for tubulin and ORF52 (Figure 3-6). In this assay, polymerized tubulin (MTs) will pellet through the cushion and be in the pellet fraction, whereas unpolymerized tubulin stays in the 
Figure 3-5. Some ORF52 has a MT-like staining pattern following RRV infection. RhF cells were infected with RRV at MOI 5 and fixed 48 hours later. Cells were stained with Dapi and anti-ORF52 antibodies. Arrow highlights area in an infected RhF where ORF52 appears filamentous and may be colocalized with MTs. Lower panel is a merged and magnified image of the single stained Dapi and ORF52 upper panels. 
146

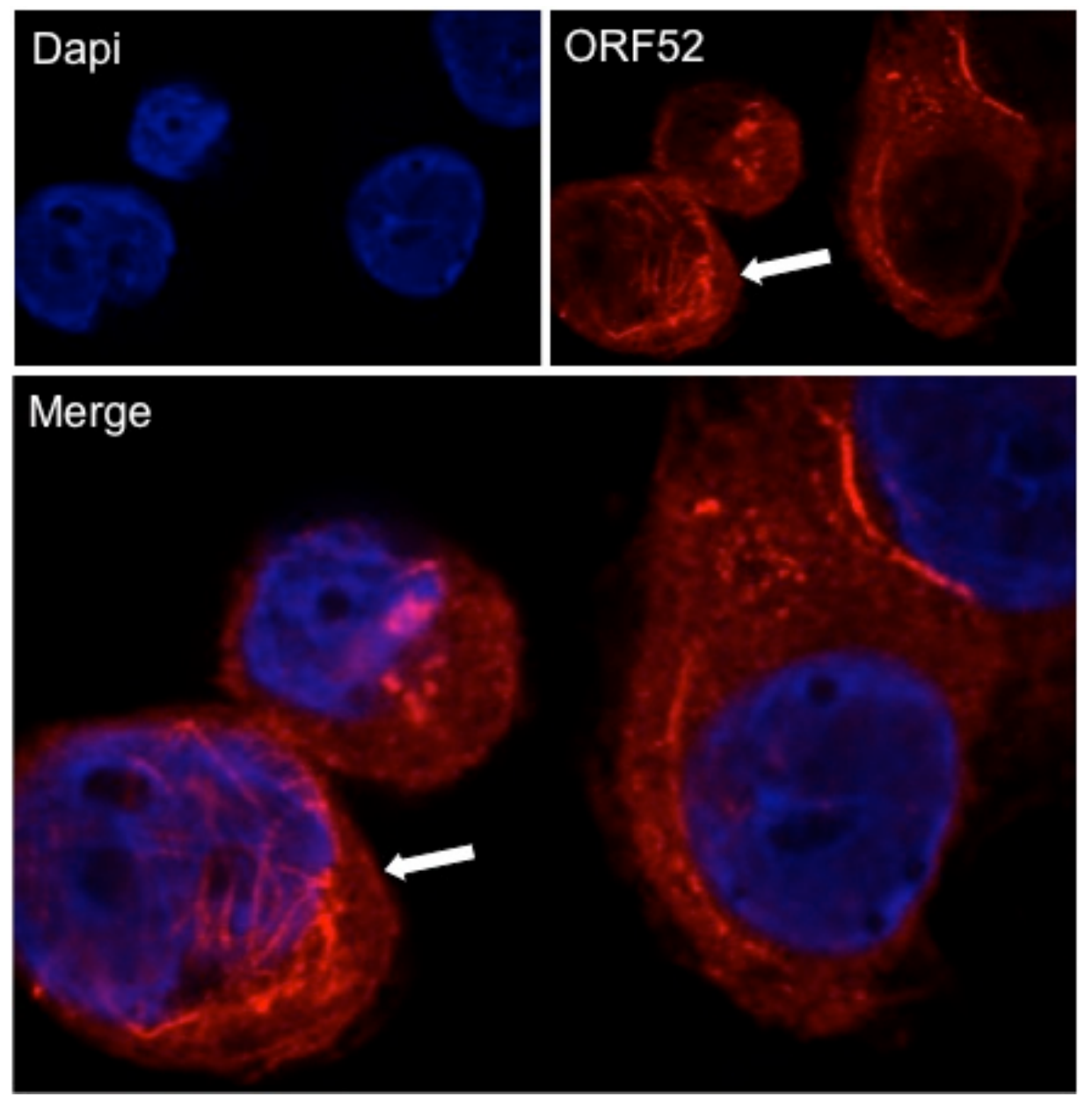


Figure 3-6. Purified ORF52 minimally associates with purified microtubules Unpolymerized or polymerized tubulin (MT) was incubated with purified RRV ORF52 protein and the suspension was centrifuged over a glycerol cushion. Western blot of fractions from unpolymerized supernatant (S) (lane 1) and pellet (P) (lane 2) and polymerized supernatant (lane 3) and pellet (lane 4) probed with anti-tubulin and anti-ORF52. 


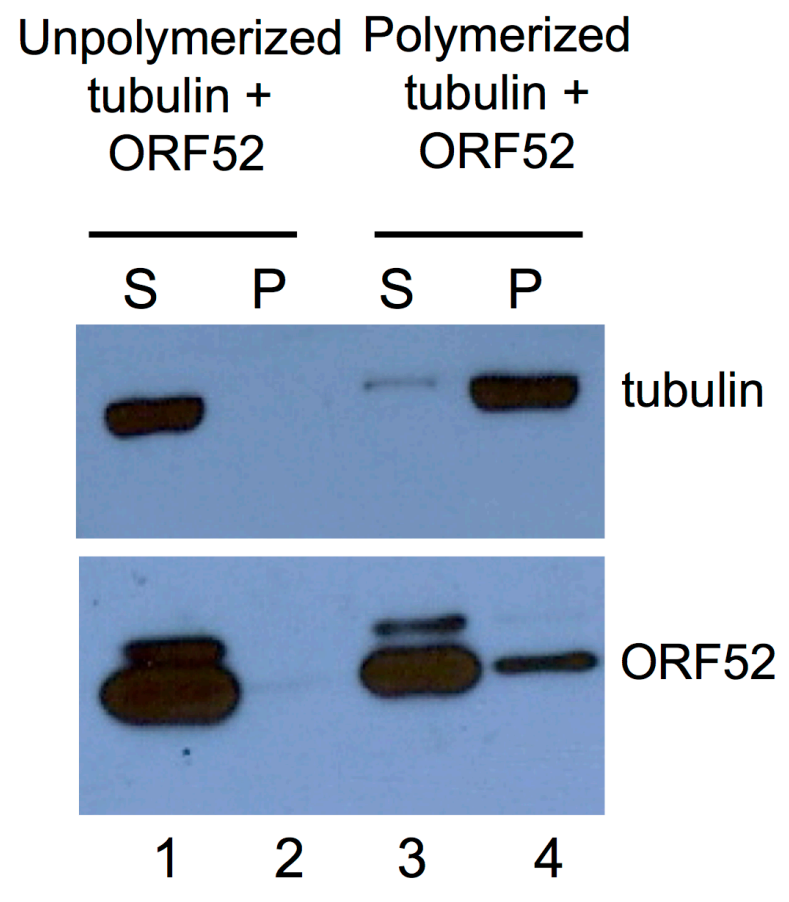


supernatant fraction. If ORF52 binds directly to MTs, it will be in the pellet fraction. Unpolymerized tubulin stayed in the supernatant (Figure 3-6, lane 1), as did ORF52 (Figure 3-6, lane 1), while polymerized tubulin (MTs) pelleted through the cushion (Figure 3-6, lane 4) along with a portion of ORF52 (Figure 3-6, lane $4)$.

ORF52 MT binding in transfected cell extracts is dependent on MT polymerization

We also examined the ability of ORF52 to associate with MTs in the whole cell context following transfection of ORF52 into both HeLa and RhF cells (Figure 37). Following a MT spindown assay whereby polymerized MTs and bound proteins would pellet, ORF52 was present in the pellet fraction with MTs at $37^{\circ} \mathrm{C}$, but absent in cold conditions: tubulin will not polymerize to form MTs on ice and, therefore, remain in the supernatant fraction following spin-down. These results suggest that ORF52 may be associated with MTs and that the association is dependent on the polymerization of tubulin (Figure 3-7).

Conserved ORF52 residue, arginine 95 (R95) (R103 in RRV), may play a role in MT thickening phenotype

Reports on the structure of MHV-68 ORF58 indicate that ORF52 self-associates through its $\alpha-2$ helix domain, functions as a homodimer, and that this association is necessary for the function of ORF52 (24). The same report suggests that ORF52 dimers are able to associate and form tetramers, via the conserved 
Figure 3-7. ORF52 MT binding is dependent on MT polymerization. (A) HeLa and (B) RhF cells were mock transfected or transfected with pkmyc-RRV ORF52. 48 hours later samples were harvested and kept at $4^{\circ} \mathrm{C}$ or at RT. Western blot of pellet fraction from cell lysates following high-speed centrifugation over BRB80 + glycerol cushion and probed with anti-tubulin and anti-ORF52. Experiment performed by former Kedes laboratory member, Sefat Kudah. 

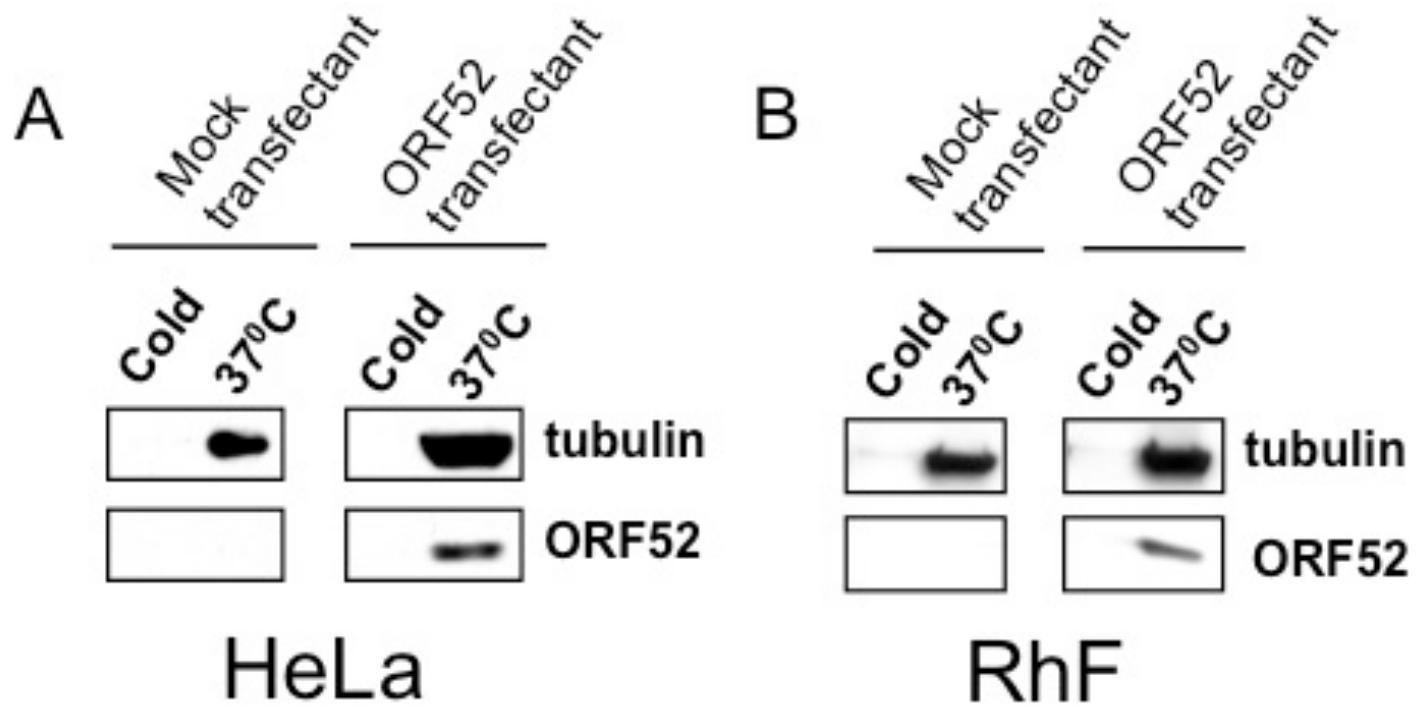
Figure 3-8. Arginine to alanine change in $\beta$-sheet of RRV ORF52 reduces MT bundling. RhF cells transfected with pkmyc-vector (panels A and B), pkmycwt ORF52, (panels C and D), or pkmyc-R103A ORF52 (lanes E and F). 48 hours post-transfection cells were fixed and stained with Dapi, anti-tubulin, and antiORF52. Experiment performed in collaboration with former Kedes laboratory undergraduate, Sophia Urban. 


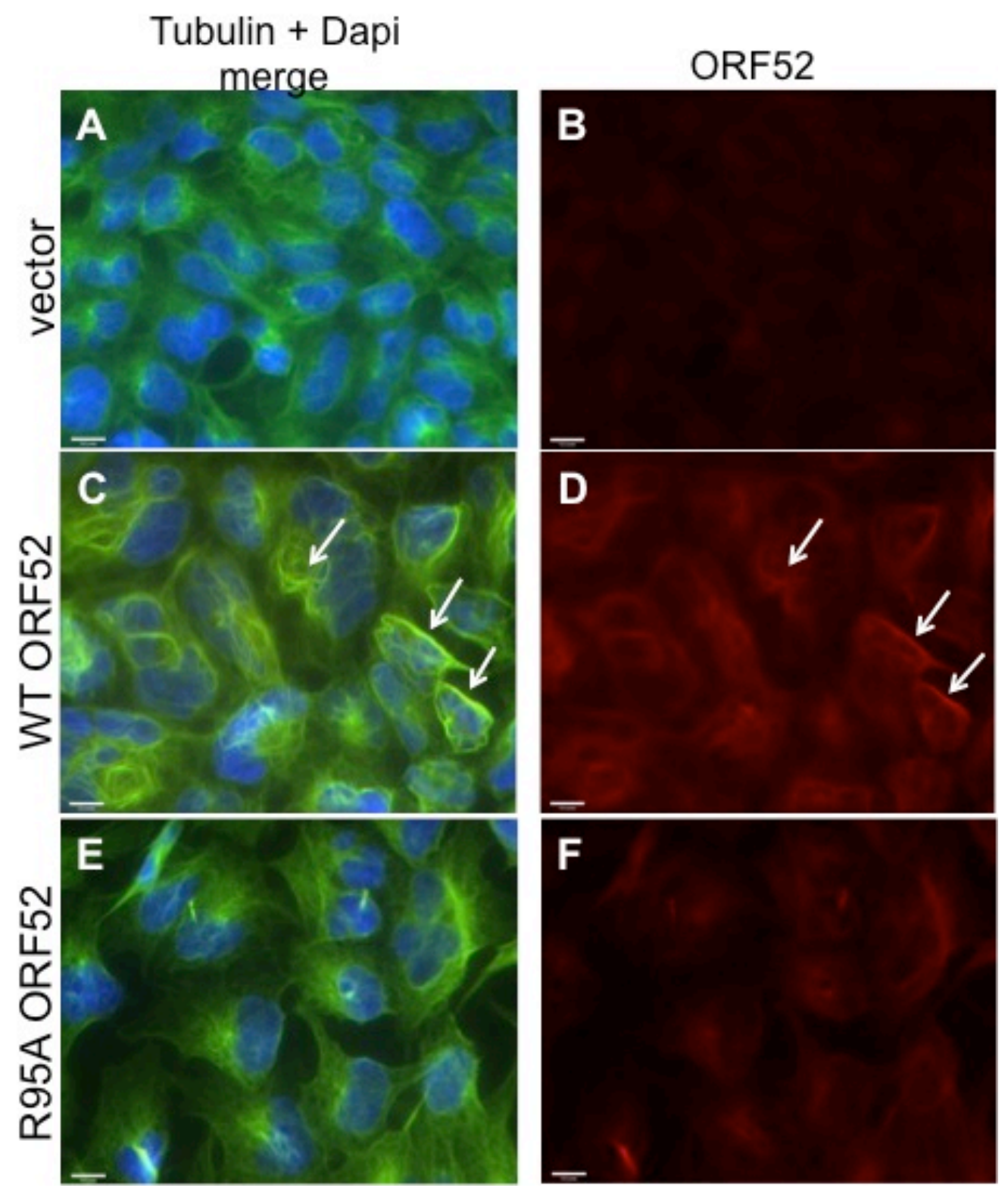


arginine 95 residue in the $\beta$-strand (24), which corresponds to amino acid 103 in RRV ORF52. We hypothesized that if ORF52 dimers were able to bind directly or indirectly to MTs, and then interact with each other by their R95 residues forming tetramers, that this might lead to a cross-linking of ORF52 tetramers bound to MTs and a thickening or bundling appearance of MTs. To test this, we made a point mutation in the conserved arginine to an alanine, hypothesizing that this would eliminate the ability of the ORF52 dimers to form tetramers, and thus, perhaps the bundle formation. We transfected HEK293 cells with vector, wt ORF52, or R103A ORF52 and 48 hours later examined the cells by IF staining for both ORF52 and tubulin (Figure 3-8). In both wt ORF52 and R103A ORF52, the ORF52 staining pattern is similar to that of tubulin (Figure 3-8, d, e, f, g). As we had seen previously, expression of wt ORF52 leads to a thickening in MTs in some of the wt ORF52 transfected cells (Figure 3-5, panel c). However, this phenotype is less pronounced in cell receiving R103A ORF52 constructs. Of note, it appears overall expression of ORF52 in the R103A transfected RhF is less that in wt (Figure 3-8, panel e). These results are preliminary, but suggest that this alteration in the conserved arginine 95 may disrupt and/or lessen thickening of MTs seen when transfected with wt ORF52.

\section{Summary}

Much of the data presented in this chapter is preliminary and at the very least needs to be repeated for confidence. The microtubule spin-down assay that utilized purified MTs and purified ORF52 might provide some insight as to 
whether ORF52 can directly interact with polymerized tubulin; however, it will also be important to consider the concentration of MTs and ORF52 combined for testing as they may not mimic the intracellular context during an infection. If we convincingly show that ORF52 does interact with MTs, it would be necessary to determine the domains of ORF52 responsible for binding MTs as well as the residues on MTs responsible for interacting with ORF52. Additionally, if we do find that R103A mutation in ORF52 alters the MT interaction profile, we would next begin trying to determine the mechanism underlying this change. If we believe it is due to the lack of ORF52 tetramer formation, we would additionally need to show that RRV ORF52 dimers do form tetramers and that this association is lost in the R103A mutation. 
CHAPTER 4: GENERAL SUMMARY AND PERSPECTIVES 


\section{Summary}

Much of the research on the structure of herpesviruses and function of herpesviral proteins has, until recently, been focused on alphaherpesviruses. However, viruses within the gammaherpesvirus subfamily have become the focus of increased interest since of all the subfamilies they have the clearest tumorigenic potential, including the ability to cause malignancy in humans as well as other animals. While many genes are homologous among the alpha-, beta-, and gamma- herpesviruses there are a number of genes found only within each subfamily and some of these proteins likely play key roles in the cellular tropism of each virus and its pathogenesis. It is important to examine differences within the subfamilies to develop a better fundamental understanding of the unique biology of each species that will, in turn, help identify novel potential therapeutic targets in each different virus species.

The central aim of my thesis was to determine the function of RRV ORF52, a tegument protein unique to the gammaherpesvirus subfamily. We conducted loss and gain of function experiments, assessing the effects on virion production during the course of a wt RRV infection. Our results indicate that ORF52 plays a critical role in the assembly of the RRV virion at a stage following viral DNA packaging and nuclear egress but preceding tegumentation and secondary envelopment. Further, our results argue that this structural protein is critical for the protein-protein interactions that permit tegument assembly, efficient transcytosis and invagination into vesicles, all of which are necessary for viral maturation and release into the extracellular space. 


\section{Discussion}

\section{The role of ORF52 in the protein-protein interactions leading to the structure of the virion}

Researchers have only an earliest stage understanding of the intricate interactions between RRV ORF52 and other proteins necessary for virion particle formation. In KSHV, investigators used a yeast-two-hybrid system and co-IP experiments and determined that ORF52 interacts with ORF45, ORF26, ORF75, gM, and gN when ORF52 was used as bait (Y2H) or antibodies directed to tagged ORF52 were used in the pull-down assay (co-IP) (267). That same study showed that, used as bait in $\mathrm{Y} 2 \mathrm{H}$ or in the pull-down of IPs, ORF45 interacted with ORF62, ORF11, ORF27, ORF33, ORF63, ORF64, ORF75, gL and gN, but not ORF52, (267). Based on this study, researchers have assembled a virion wide protein interaction map for KSHV (Figure 4-1). The figure illustrates the multiple direct and indirect interactions between ORF64-ORF52-ORF33ORF45, as well as the interaction between ORF52-ORF75-ORF45 (Figure 4-1). In the KSHV interaction study (267) as well as outlined in Figure 4-1 (275) ORF52 and ORF45 directly interact. In our studies with RRV, we were unable to detect a direct interaction between ORF52 and ORF45, when either was used in co-IP experiments following co-transfection, nor were we able to find an indirect interaction following IP in RRV infected RhF cells (data not shown). This is confusing to us, as we have evidence that knocking down expression of ORF52 affects the ability of ORF45 to be packaged within the virion as shown by western blot and IF (see Chapter 2, Figure 2-3). 
Figure 4-1. Interactome map of KSHV virion proteins. Interactions amongst the virion proteins, capsid (inside the hexagon), tegument (outside the hexagon but within the oval), and envelope (outside oval) are indicated with arrows. Image from (275), an open-access article. 


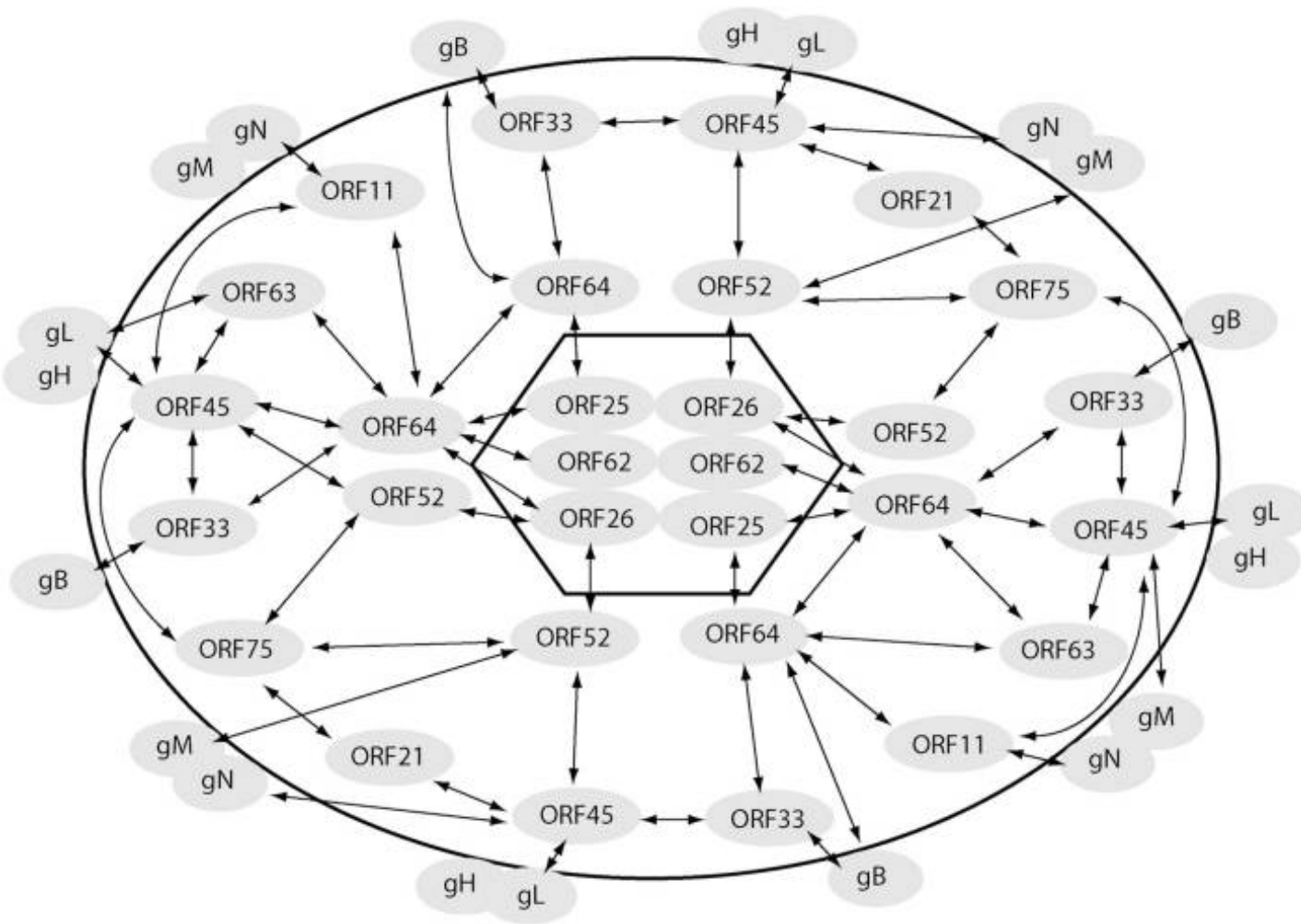


KSHV ORF33 pulled-down ORF52 when used in a co-IP experiment (267). ORF33 is conserved among the herpesviruses and in HSV-1 the homolog UL16 may function in viral DNA packaging, virion assembly, budding and egress (16, 183, 204, 224, 242). In MHV-68, when researchers transfected 33STOP BAC into 293T cells, intracellular particles retrieved by freeze thaw of cells showed particles that lacked ORF33 and ORF45, but that had ORF52 at levels similar to wt BAC (120). In cells transfected with 33STOP BAC, particles were unable to undergo secondary envelopment and egress (120). This suggests that ORF52 may be more closely associated with the capsid than ORF33 and that ORF52 may provide a scaffolding-type function where its expression and addition to the particle is required for addition of other proteins such as ORF33 and ORF45. This is consistent with the proposed interaction map in KSHV (Figure 41). However, in the discussion of MHV-68, Wang et al. did not mention ORF33 interacting with ORF52 (330), so this interaction may or may not occur in MHV68 and/or RRV.

The KSHV protein-protein interactions map (Figure 4-1) and co-IP data examining interactions between different proteins (267) indicate a direct interaction between ORF52 and ORF45 and suggest ORF33 may interact with ORF45 or the conserved large tegument protein (LTP), ORF64. ORF64 (HSV-1 UL36 homolog) is an inner tegument protein that was present in both ORF45 and ORF75 IPs in KSHV (267). In MHV-68, ORF33 colocalizes to cytoplasm and nucleus, but in the presence of another tegument protein, ORF38, ORF33 localizes to TGN. ORF33 co-localizes with ORF38 during viral infection. ORF38 
is also a conserved tegument protein, (UL11 in HSV-1), that in MHV-68 localizes to the cytoplasm during transient transfection and infection and is packaged into virions during secondary envelopment (286). The interaction between ORF33 and ORF38 in MHV-68 is conserved in HSV-1 between UL16 and UL11 (346).

When used as bait in KSHV during yeast-two-hybrid experiments, or to pull-down in co-IP experiments, another gamma-specific tegument protein, ORF75, interacted with ORF62 (TRI-1), ORF21, ORF64M, gM, and gN. ORF75 interacts with ORF52 and ORF45 only when ORF75 was the prey (267). ORF75 is a member of the FGARAT family of proteins involved in counteracting the ND10 cellular restriction factors that inhibit herpesvirus Iytic DNA replication. The introduction of this dissertation provided information on this function in greater detail.

As can be garnered from the above section, the interactions between viral proteins are quite extensive, with evidence for specific interaction between proteins in KSHV—such as between ORF52 and ORF45-that have not been found in RRV, yet need to be further investigated. Homology in the viral proteins across the subfamilies may speak to a conserved function or partial function; however, remaining variance could account for different protein-protein interactions and functional variance. For example KSHV ORF45 is found in the cytoplasm, but MHV-68 and RRV ORF45 are found in the nucleus, and this difference in localization may alter the order in which ORF45 is added to the particle, thereby affecting addition of subsequent proteins. Study of RRV has not 
yet elucidated the interaction between any of these proteins and, in order to determine what is happening in RRV, investigators must further assess and tease out these interactions.

In the future we would like to investigate the potential protein-protein interactions necessary for virion maturation by creating a library of differentlytagged RRV proteins that we would co-transfect into permissible cell lines such

as HeLa or 293 and IP for interactions following detection by western blot. Special attention would be paid to potential interactions between ORF52 and the capsid protein, ORF26 (TRI-2) implicated in KSHV, as well as between the tegument proteins ORF64, ORF33, and ORF75. Interactions discovered from these studies will provide an overall map of interactions in RRV, as well as speak to conservation between RRV and KSHV and/or MHV-68.

\section{When is ORF52 added to the particle?}

Based on detergent sensitivity and mass spectrometry (MS) (239) ORF52 is a tegument protein tightly associated with the capsid; however, researchers do not yet know when ORF52 is added to the tegument. We have no evidence that ORF52 is ever in the nucleus - it has no NLS and there is no nuclear ORF52 staining by IF - but if it is an inner tegument protein that forms an essentially base-like layer on the capsid for binding of other proteins, (which seems likely based on the KSHV map, MHV-68 data, and our own observations), we would hypothesize that it is added to the particle soon after nuclear egress into the cytoplasm, thus allowing other proteins to bind. 
Unfortunately, immuno-EM images in MHV-68 are not entirely clear and show ORF52 present on released virions, within the cytoplasm located near the Golgi, in the membrane of vesicles, on particles within vesicles, and on immature particles not yet within vesicles (82). While ORF52 is added to the capsid in the cytoplasm, looking solely at the immuno-EM in the MHV-68 study, it is difficult to determine if ORF52 is added at multiple places within the cytoplasm (i.e. prior to, and again during, secondary envelopment) or if ORF52 addition is required at one stage and disposable at another. Currently, we do not know precisely when RRV ORF52 is added to the tegument layer of egressing herpesvirus particles.

To investigate this matter, we propose performing immuno-EM during RRV infection, in both wt and ORF52 kd conditions and looking for ORF52 staining by TEM. Since we have an antibody directed to RRV ORF45, we could additionally perform immuno-EM to determine when ORF45 is added to the particle, a matter that is of interest to our laboratory.

\section{The role of ORF52 in secondary envelopment}

Researchers have a more limited understanding of the specific proteins involved in secondary envelopment - as well as the events that trigger this process - than one might expect. Investigators have documented a number of interactions between capsid-tegument, tegument-glycoprotein, and capsid-glycoprotein proteins (reviewed in (208)) in the herpesviruses, yet the location of secondary 
envelopment, and whether that location varies depending on the individual herpesvirus is still unknown (reviewed in $(119,126,212,213)$ ).

Research on HSV-1 suggests that secondary envelopment occurs at the TGN, with documented interactions between the tegument proteins pUL16 (ORF33), pUL11 (ORF38), and pUL21 (ORF24) (162, 303, 346). pUL11 accumulates at TGN-derived vesicles, which may enable recruitment of other required proteins to these vesicles (182). Investigators have also found interaction between ORF33 and ORF38, and the localization of ORF38 at TGN vesicles, in MHV-68 (286). If researchers delete any of these proteins, there are egress defects and an accumulation on non-enveloped capsids in the cytoplasm $(27,120,145,164,278,283,286,290,330) . H S V-1$ studies have also shown an interaction between the tegument proteins pUL48, pUL41, pUL46, pUL47, and pUL49 with evidence that pUL49 interacts with the cytoplasmic tail of the glycoproteins gE, gD, and US9-possibly shedding some light on the interactions between tegument proteins and glycoproteins that might stimulate secondary envelopment. However, there are no homologs for the above-mentioned tegument proteins in RRV, so identifying the tegument proteins necessary for secondary envelopment remains an area open for investigation and one of great interest to our laboratory. In RRV infected RhF cells co-stained with antibodies directed to the TGN and the viral capsid protein SCIP, we were unable to detect colocalization (data not shown). Our results suggest that RRV may undergo secondary envelopment at a non-TGN derived vesicle, and we are currently exploring this option, looking for markers to distinguish early endosomes (EE), 
late endosomes (LE), recycling endosomes (RE), and plasma membrane surface derived vesicles.

\section{Domains of ORF52 responsible for its functions in both the RRV life cycle and MT interaction}

Structural information on MHV-68 indicates that ORF52 is composed of $3 \alpha$ helices followed by a C-terminal $\beta$-sheet (24). In MHV-68 the $\alpha-1$ domain is responsible for localization within the cell, the $\alpha-2$ domain for dimerization and self-association of ORF52, and the conserved R95 residue in the C-terminal $\beta$ sheet for interaction with ORF42 (330). We found by western blot following IP of both transfected and RRV infected cells that RRV ORF52 also self-associates (data not shown). We showed that RRV ORF52 is necessary for virion maturation and that without it there is a significant decrease in the amount of infectious virus released, although that amount could be partially rescued by providing ORF52 in trans (Chapter 2, Figure 8). However, the domain responsible for the rescue is not known, nor is the domain responsible for MT interaction. To investigate domains required for function, we would make different mutations to our siResistant ORF52 plasmid and transfect it into RRV infected cells treated with siORF52. We would determine if these mutant constructs were still able to rescue virus production by calculating viral titer and released wt virion determined by protein production. We would also examine the localization of mutant ORF52 by IF and determine its ability to self-associate by IP. 


\section{Release of subviral particles in ORF52 kd}

In MHV-68, cells transfected with 52STOP BAC did not release particles or virions (24). In our system we used a knockdown instead of a knockout, so we did expect to see some released wt particles from cells that did not receive siRNA but were infected with RRV. However, in our experiments we found that there were approximately the same amount of capsids released in the ORF52 kd determined by MCP and SCIP, and that more than $50 \%$ were empty A capsids (Chapter 2, table 2-1). We hypothesized that a passive mechanism released these capsids from the cells upon lysis of the cell and that any composition of particles $(A, B, C)$ in the cells would be released. Upon enumeration of the different intracellular particles, our hypothesis was not proven true because we identified many more DNA-filled C capsids in the cells than the numbers in the released particles would suggest (Chapter 2, table 2-1). We also looked at the conditions 24 hours p.i. and, while the total number of capsids released was less than at $48 \mathrm{~h}$ p.i., it was still relatively similar between siCNL and siORF52 (data not shown). Additionally, we observed more capsids produced and released in the siCNL than expected- $24 \%$ including A, B, and C capsids (Chapter 2, table 21). We suspected the increase in the proportion of empty A capsids collected from the supernatant in siORF52-treated RhF cells might be due to ORF52 playing a role DNA retention in the capsid. In HSV-1 the proteins encoded by UL17 and UL25 manage DNA packaging and retention (58, 230, 310, 313, 316) (ORF32 and ORF19 respectively in RRV/KSHV). 
We hypothesized that, as an inner tegument protein, ORF52 may be in proximity to interact with ORF32 and/or ORF19 and that, with it absent in the kd conditions, the DNA may not be as securely packaged. Furthermore, following our high-speed centrifugation protocol to separate particles, we posited that DNA might simply escape, resulting in a large number of A capsids in the supernatant that were actually DNA filled C capsids. We tested this by isolating capsids from the cytoplasm in kd and siCNL and subjecting them to the same centrifugation protocol and subsequent examination by TEM as our released supernatant samples. We already showed that the majority of capsids in the siORF52 cytoplasm (Chapter 2, Figure 2-6D, 2-8D, and Table 2-1) had DNA, so if they were more fragile and/or the DNA was packaged less securely because they lacked ORF52, following isolation and centrifugation, we would expect to see a large number of empty capsids that had lost their DNA in our released sample. However, our results contradicted this prediction and instead we found the vast majority of capsids to have DNA (data not shown). We have yet to determine the mechanisms of release for these subviral particles or why the majority of capsids are empty. We speculate that there might be a loss or alteration of nuclear or plasma membrane integrity in the ORF52 kd that allows for escape of subviral particles.

\section{Role of ORF52 in interaction with MTs and movement of particles}

Upon overexpression of ORF52, we noticed that ORF52 appeared to have a staining pattern similar to microtubules and frequently created a bundled or 
thickening appearance in MTs (Chapter 3, Figure 3-1, 3-2, and 3-3). We have also observed this MT-like staining pattern in RRV infected RhF cells, albeit to a lesser extent than in transient transfection experiments. Our preliminary data suggest that ORF52 associates with MTs during purified pull-down experiments, as well as following transfection of ORF52 and MT pull-down of cell lysates; however, we have yet to fully explore this potential relationship. While research in the field has well documented the fact that herpesviruses utilize the MT network for both anterograde and retrograde transport $((76,225)$ and reviewed in $(74$, 116)) following entry into the cell and during egress, the potential advantage for MT bundling upon infection or transfection is not known. It may stem from the mobility of the virus, suggesting bundling occurs not as a negative side effect but during movement of the viral particle. RRV ORF52 is not the only herpesvirus tegument protein to demonstrate this type of phenotype. In HSV-1, VP22 is a tegument protein with properties similar to cellular MAPs, in that it colocalizes with MTs and reorganizes them into thick bundles that are highly stable and resistant to depolymerization (86).

While ORF52 may interact directly or indirectly with MTs, it is important to consider the potential interaction between RRV ORF45 and motor proteins, as documented in KSHV where ORF45 docked the viral capsid onto the kinesin-2 motor protein KIF3A (277). In KSHV, ORF45 is cytoplasmic, whereas it is nuclear in RRV (and MHV-68), unless associated with maturing viral particles in the cytoplasm or in virions following release. Thus, this relationship between ORF45 and KIF3A may not extend to RRV. 
In the future we would like to repeat our experiments involving transfection of ORF52, followed by staining with tubulin using different ORF52 mutant constructs. We would transfect cells with ORF52 mutants including $a-1$ helix, $\alpha-2$ helix and $\beta$-strand deletions, as well as mutations of phosphorylated serine residues to alanine, and examine the localization to see if the pattern changes. Additionally, we would determine if MT depolymerization alters localization of ORF52 and test with anti-acetylated tubulin antibodies to examine whether MTs are stabilized in wt or ORF52 kd and if that changes with use of different mutant constructs.

\section{Phosphorylation of ORF52 and interaction with SRPK2}

Serine/arginine rich kinase 2 (SRPK2) interacts with, and phosphorylates, EBV BLRF2 at a conserved RS motif within the C-terminus (noted in Figure 1-9 in the introduction) and this phosphorylation plays a role in viral replication. Upon mutation of serines 148 and 150 to alanines (ARA) in BLRF2, this mutant was still able to associate with SRPK2 as shown in IPs; however, it was unable to complement the defect in viral replication measured by viral DNA copies in the supernatant (82).

SRPK2 is discussed in greater detail in the introduction, but it is interesting to note that SRPK2 also phosphorylates the MAP, tau, at S214. Tau is a wellstudied MAP that mediates cognitive defects in Alzheimer's. Tau has an Nterminal projection domain, a C-terminal of MT binding domain repeats, and a short tail. The phosphorylation status regulates binding of MTs and SRPK2 
phosphorylation of tau at S214 suppresses MT polymerization (134, 136, 152). RRV ORF52 is phosphorylated at three residues: serines 46 or 48,107 and 119 (Woodson and Kedes, unpublished data). While the homologs do not strictly conserve the C-terminal RS domain (Figure 1-9, introduction), it would be interesting to determine if SRPK2 phosphorylates RRV ORF52, if that phosphorylation is necessary for the function of ORF52, and if so, for which function - tegumentation, egress, or MT interaction. If phosphorylation by SRPK2 of ORF52 does take place, it would provide an additional similarity between a well-established cellular MAP like tau and this viral tegument protein.

\section{Creation of a RRV BAC - wt, 52-stop, 52-revertant}

To more cleanly demonstrate the function of other RRV viral proteins and their role in the virion structure, it may be useful to create a functional RRV BAC system that has an antibiotic selection marker and either a fluorescent tag or a FLAG/HA/myc tag. Once investigators create and test the wt BAC they can then perform genetic manipulations to introduce stop codons into the specific gene of interest, and subsequently create a revertant BAC again expressing the gene of interest. While we believe there are positive reasons to use the high efficiency siRNA knockdown during wt RRV infection and rescue system that we used to investigate ORF52 in Chapter 2, including use of wt virus during a primary infection in a biologically relevant cell type, this approach also has its difficulties and detractors. The most widely studied herpesvirus genomes (HSV-1, CMV, KSHV, MHV-68) are cloned as BACs $(4,51,87,153,174,207,223,304,328$, 
$347,355)$ and researchers have made many different genetic manipulations and additional BACs for use in investigations. There is potential concern that a knockdown (siRNA), as opposed to a complete genomic knockout using a BAC, may muddle the results by giving a mix of results from wt virus infected/not knocked down, as well as knockdown, cells. We are currently in the process of creating lentiviral vectors that we can use to express a tagged wt ORF52 or siResistant ORF52 to transduce RhF cells during siRNA knockdown and wt RRV infection experiments. We believe this is a promising way to utilize our current system, while increasing ORF52 expression and the health of the cells, i.e., by not using Amaxa for plasmid transfection. Once established, researchers could manipulate this lentiviral expression system with relative ease to express different RRV genes for further examination of other viral proteins such as ORF45, ORF75, ORF33, and ORF64.

\section{Does RRV ORF52 play a role following virus entry?}

Upon viral entry the viral envelope fuses with a cell membrane (either the plasma membrane or a vesicle membrane following endocytosis) and releases the capsid and tegument proteins into the cytoplasm $(113,195)$. At this point the majority of tegument proteins dissociate from the capsid $(113,114,195)$ and can play roles in priming the cell for viral infection (294). In HSV-1 and PrV, the inner tegument proteins UL36 (ORF64 homolog), UL37 (ORF63 homolog), and US3 (not in gammaherpesviruses) remain associated with the capsid following entry and transport to the nucleus $(60,76,195,245,256,336)$. ORF52 is a tegument 
protein that appears to colocalize with MTs and may bind to MT and/or play a role in particle movement. It would be interesting to investigate whether ORF52 remains capsid-associated during entry and whether it functions in capsid translocation to the nucleus. To explore this, we could use immunoEM staining of ORF52, following high MOI infection at early time points, for example 2, 4, 6, $8 \mathrm{~h}$ p.i. If ORF52 remains capsid-associated, we would then investigate whether it plays a role in transport toward the nuclear pore utilizing MTs. Using IF and IP, we may be able to determine if ORF52 colocalizes or interacts with dynein motors during entry.

\section{The tegument and its potential role as a therapeutic target}

In addition to our goal of investigating the role of ORF52 in the RRV life cycle, we also hoped our work would inform investigation into other herpesviruses, specifically KSHV, as these two gamma-2 herpesviruses are closely related. Our overarching hope was that we would be able to identify ways to block gammaherpesvirus lytic replication and, thus, decrease the disease burden associated with this virus family. ORF52 is a conserved gammaherpesvirus specific protein, making it an attractive target for specific inhibition that would not hit cellular targets, but might work to inhibit both KSHV and EBV (both pathogenic human herpesviruses). ORF52 is likely involved in two separate

critical stages of the viral life cycle—de novo infection and lytic replication-and it may be a broadly acting target. Upon in vitro knockdown of ORF52, subviral capsids were released from the cell, yet were over 300 -fold less infective than 
RRV released in siCNL (Chapter 2, Figure 2-1C). This release of less infectious, defective, unenveloped subviral particles and intracellular accumulation of viral proteins may also have the potential to generate an immune response without the highly lytic nature of a wt infection. In addition to ORF52 as a potential target, ORF45 might also provide a potential therapeutic target, for many of the same reasons as ORF52. In terms of potential target development, small molecule screens or rational drug design, followed by combinatorial chemistry type approaches could be used to search for an ORF52- (or ORF45-) specific inhibitor. Since tegument proteins are not surface expressed in wt virions, immunotoxins would not be effective; however, they are highly expressed in lytic cells and may be potential vaccine targets.

\section{Model of virion maturation with, and without, ORF52}

Our results show that when ORF52 is knocked down, capsids containing DNA egress the nucleus without significant deficit (Figure 2-6D and Table 2-1). Upon examination these capsids appear to lack tegument, although they may contain a minimal amount of protein not clearly visible by TEM (Figure 2-6D) suggesting the necessity of ORF52 for the continued layering of tegument proteins to take place. Additionally, IF results indicate that ORF52 is necessary for nuclear egress of the outer tegument protein ORF45 (Figure 2-4). In KSHV ORF45 interacts with the kinesin-2 motor protein docking the tegumented capsid for movement along MTs (277). Without ORF52, ORF45 appears unable to be added to the capsid (Figure 2-4). In attrition, in ORF52 knock down cells, capsids 
may be unable to efficiently interact with motor proteins, like kinesin-2, and be moved along MTs toward the plasma membrane. In summary, we submit the following model to illustrate the role of ORF52 in the maturation of RRV (Figure $4-2)$. 
Figure 4-2. Model highlighting impact of ORF52 knockdown and its effect on virion maturation. (A) RRV maturation in the presence of ORF52. (a) Capsid egresses the nucleus, (b) obtaining inner tegument proteins (brown and green circles), including ORF52 (green) that might possibly interact with motor proteins such as Kinesin-2, MTs directly, or other cellular/viral proteins (not shown). (c) Outer tegument proteins are added to the particle (orange, brown, and blue), followed by (d) secondary envelopment of glycoprotein containing vesicles. Particles within vesicles are transported toward the cell surface by motor proteins, possibly Kinesin-2, that may require other cellular/viral proteins (not shown). (f) The vesicle fuses with the plasma membrane and virions are released and either stay cell-surface associated or disperse into the media. (B) When ORF52 is knocked down, (a) capsids exit the nucleus, and potentially some inner tegument proteins that do not require ORF52 expression are added to the particle (brown circles). (c) Knock down of ORF52 prevents outer tegument proteins that require ORF52 expression, potentially as binding partners, to be added to the particle. (d) Partially tegumented/untegumented capsids that are impaired for secondary envelopment, rosette around vesicles, unable to undergo envelopment. Of note, without proper tegumentation, particles may be unable to associate as needed with motor proteins and may not move throughout the cytoplasm with the same efficiency as wt and remain closer to the nucleus. (e) Subviral particles, the majority of which do not have DNA, and lack tegument proteins, including ORF45 and ORF52, as well as envelope, are released, by a yet unknown mechanism, into the media. 

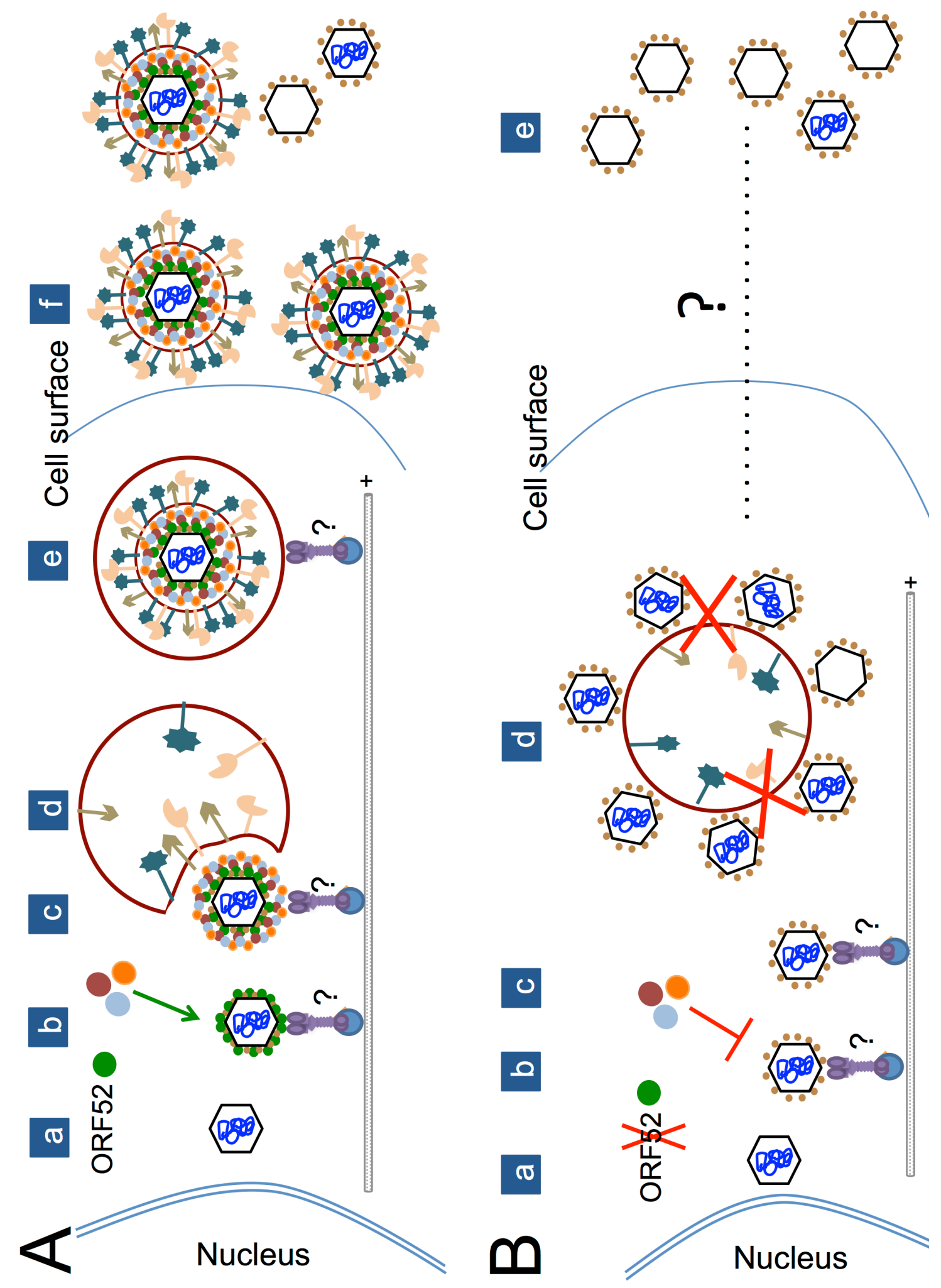
APPENDIX 1: RRV BACTERIAL ARTIFICIAL CHROMOSOME (BAC) 
In our early investigations into the role of ORF52 in the RRV life cycle we initially hoped to utilize bacterial artificial chromosomes (BACs) containing either the wt RRV strain 17577 genome or an ORF52 deletion ( $\triangle 52$ BAC) (gifts of Scott Wong at the Oregon Regional Primate Research Center, created in his laboratory). Our plan involved transfecting RhF cells with either wt BAC, $\Delta 52$ BAC, or complementing the $\triangle 52$ BAC by providing exogenous ORF52 plasmid by transient transfection. We would allow time for activation of the viral lytic life cell and formation of cytopathic effect (CPE), and utilize the samples for our experiments narrowing down the function of ORF52 by what changed in the virus life cycle without it.

Numerous herpesvirus genomes have been cloned as BACs, including murine CMV, HVS, MHV-68, rhesus CMV, EBV, HCMV, VZV, HSV-1, KSHV, and $\operatorname{RRV}(4,51,87,153,174,207,223,304,328,347,355)$. BACs allow for entire viral genomes to be sustained in $E$. coli and, following transfection into eukaryotic cells, production of progeny virions. BACs allow researchers to examine the function of specific viral proteins in the course of infection by alteration, mutation, or deletion of sequences encoding a specific viral protein. Care needs to be taken when designing BACs, as manipulations pose potential concerns and technical issues, especially for proteins with overlapping coding regions and sequences left over from cloning (reviewed in (3)).

The utilization of a BAC also allows investigators to make recombinant viruses. This includes viruses that express a fluorescent marker fused to a specific protein of interest, such as GFP, for easier immunofluoresecent imaging 
of a viral capsid protein during the course of infection, or a tag such as FLAG, and/or to make viruses that lack the coding sequence for particular proteins useful for examining function of those proteins.

The Wong laboratory tested the wt BAC derived RRV and found it similar to wt (non-BAC derived) RRV in terms of infectious virions produced (87). The $\triangle 52$ BAC was not tested for functionality following creation and gifting to our laboratory.

To obtain BAC DNA, we grew large clonal E. coli cultures, containing either wt or $\triangle 52 \mathrm{BAC}$, and purified DNA using phenol-chloroform-isoamyl alcohol and ethanol precipitation. Once BAC DNA was isolated, we tested the preparation to ensure DNA was present and to determine potential integrity by cutting it with HindIII restriction enzyme and running a sample on an agarose gel (Figure A1-1).

To test the BAC DNA for functionality, we first transfected subconfluent ( $70 \%)$ RhFs with 1-2 $\mu \mathrm{g}$ of BAC DNA with Translt transfection reagent (Mirius) and checked the cells daily for evidence of CPE. In the wt BAC DNA transfected cells, depending on the DNA preparation, we saw evidence of CPE at the earliest, 8 days post DNA transfection, but on average it was 10 or more days (Figure A1-2). This is in stark contrast to primary RRV infection where CPE is evident and infectious virions can be collected from the supernatant 24 hours post infection. This extensive time period between transfection and CPE posed immediate concerns in terms of using the BAC for my research purposes. For 
Figure A1-1. BAC DNA purified from E. coli. wt BAC and $\triangle 52 \mathrm{BAC}$ cut with Hindlll restriction enzyme and run on a $0.8 \%$ agarose gel. 


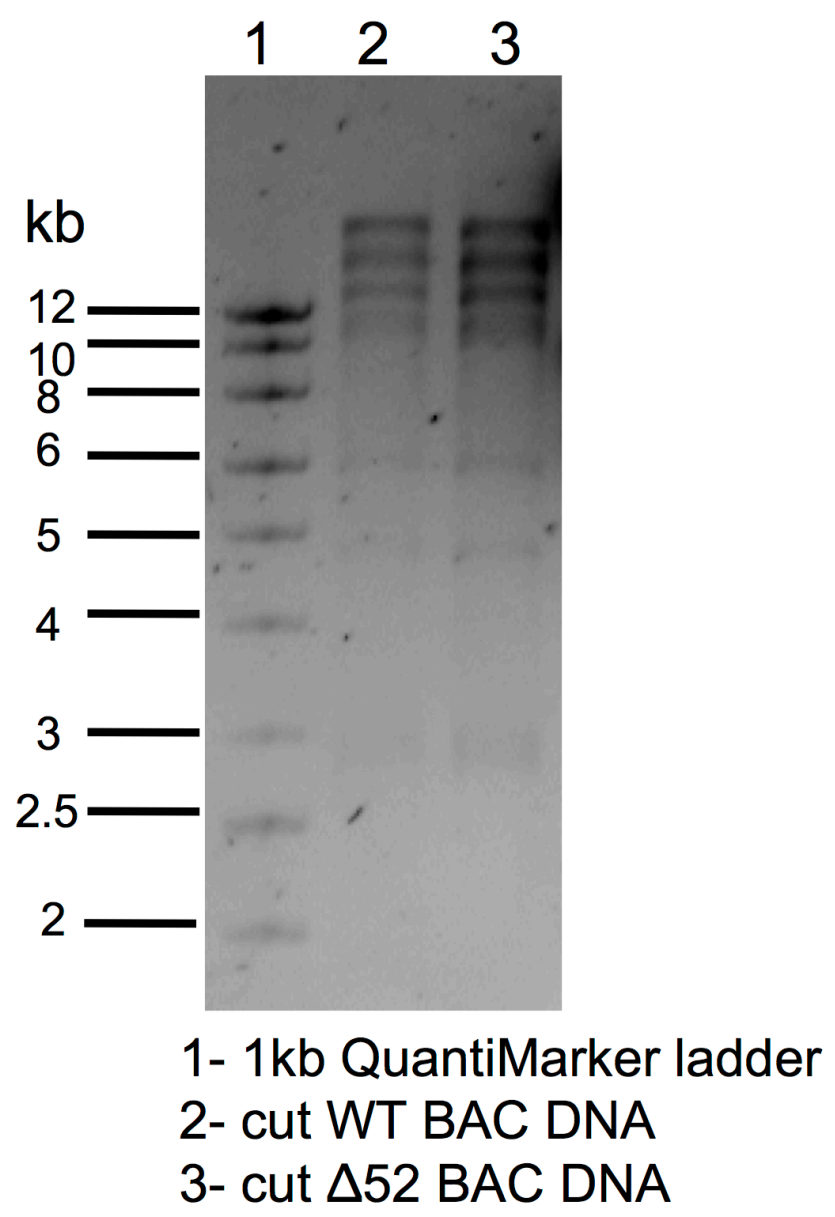


Figure A1-2. Lack of CPE evidence in RhF transfected with $\triangle 52$ BAC.
A) Untransfected RhF, B) RhF transfected with wt BAC for 10 days, C) RhF transfected with $\triangle 52 \mathrm{BAC}$ for 10 days. Only RhF transfected with wt BAC show evidence of CPE as indicated by arrow. 

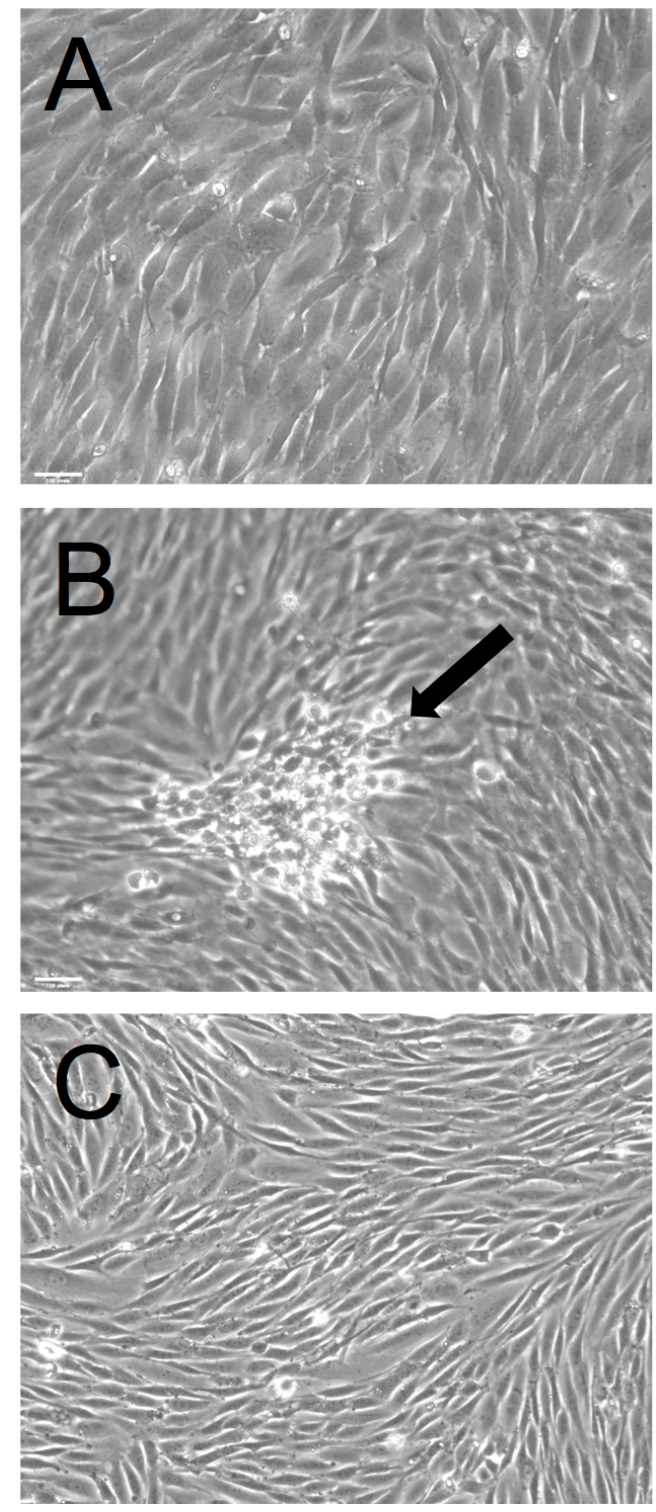
example, following BAC transfection, the host cells continue to divide, doubling approximately every 24 hours. If it takes 10 days for evidence of viral production - not robust viral activity, but any indication of activity - maintaining the cell cultures while keeping the transfected DNA concentrated within the cells to produce virus is highly unlikely. Additionally, if the wt BAC takes 10+ days from transfection to viral production, complementation of the $\triangle 52$ BAC poses even greater challenges, as these cells needed to be transfected with plasmid ORF52, which is not expressed 10 days after transfection and is also being diluted out in dividing cells.

We initially assumed that the $\triangle 52 \mathrm{BAC}$ was functional in all ways, except for deletion of the ORF52 coding sequence so any phenotypic differences between the wt BAC and the $\triangle 52$ BAC would be attributable to the lack of ORF52. If ORF52 were critical in the RRV life cycle and in production and/or release of infectious virus (which we show it is in Chapter 2 of this thesis) we wouldn't expect to see CPE or virus production in $\triangle 52 \mathrm{BAC}$, which we never did. Unfortunately, in over 2 years of troubleshooting and optimization of the BAC in our laboratory we were never able to complement the ORF52 deletion by providing ORF52 in trans under any conditions. We never saw any suggestion of CPE or viral production following complementation with $\triangle 52 \mathrm{BAC}$.

While this may be due to factors such as the amount of plasmid ORF52 transfected, the timing of plasmid addition, the loss of plasmid, the dilution of BAC DNA during splitting of cells over the course of $10+$ days, it became clear that it was impossible for us to narrow down in a way that was useful for our 
research purposes. Of important note, we were also never able to detect any viral DNA present by PCR in $\triangle 52$ BAC transfected cells, which we would expect if the BAC was functioning properly, because ORF52 expression is not required for viral DNA replication or expression of SCIP, MCP, and ORF45 (see Chapter 2, Figure $2-1$ and $2-2 A$ ). Therefore, it is quite probable that there were functional issues with the $\triangle 52 \mathrm{BAC}$ and that even under optimal complementation conditions, rescue would not have happened. For example, there were several sequences left behind from the cloning and deletion of ORF52, including plasmid and primer sites (Figure A1-3) that may have posed additional issues.

There were several challenges that presented themselves regarding using the BAC. 1) Expression of viral proteins is highly regulated. Specifically, the point at which each protein is expressed, and/or whether it is expressed at different stages in the viral life cycle is difficult to determine and therefore when we provide plasmid to the cells may not be when it is needed, 2) The amount of protein expressed also varies and in some cases with exogenous transfection the amount may not be too little or too much. For example, we have repeatedly shown that overexpression of ORF52 can lead to microtubule bundling, which may pose a potential hindrance to viral infection, 3) RhF cells are difficult to transfect under the best of circumstances. Transfecting a large BAC into the cells via Translt resulted in approximately $10-30 \%$ transfection efficiency, made additionally difficult by number 4,4 ) the BAC did not have an antibiotic selection marker. I was unable to select for cells that received the BAC, which potentially may have assisted in reducing the period of time from transfection to viral 
Figure A1-3. Sequencing alignment of $\triangle 52$ BAC compared to wt RRV H2595 ORF52. Lower sequence in purple is the original wt 420 bp RRV ORF52 sequence $(\mathrm{NCBI})$, with the start (ATG) and stop (TAG) in red. The upper sequence is from the $\triangle 52 \mathrm{BAC}$ and shows various sequences remaining in the genome from the construction of the $\triangle 52 \mathrm{BAC}$. In yellow is a portion of $\mathrm{p} 73$ plasmid sequence, in green a FRT site sequence, and in blue M13 primer. (See text for addition description.) 


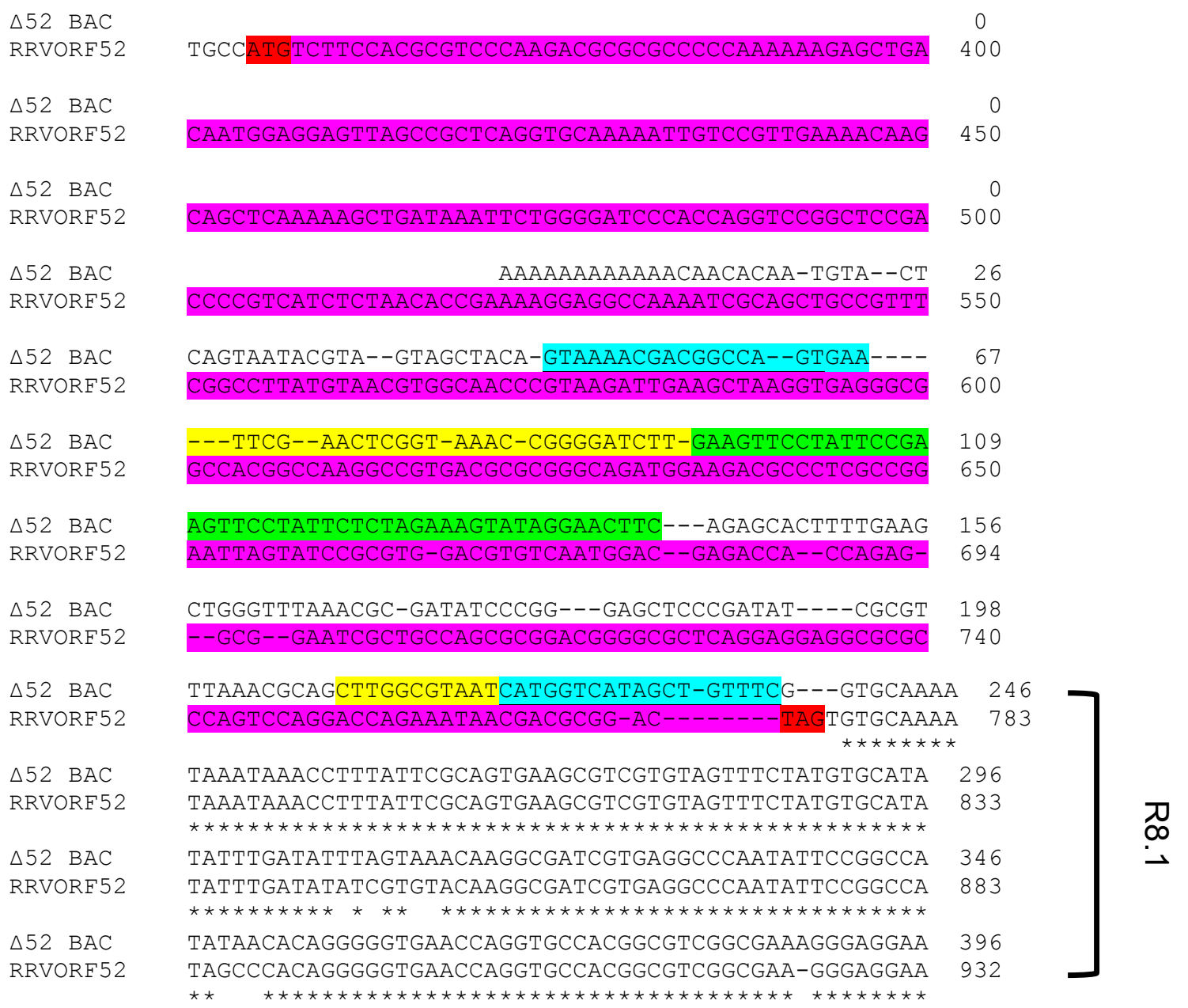


production, and 5) The doubling of RhFs every 24 hours, which meant they had to be split throughout the course of the experiment, which in turn diluted the BAC DNA and in the case of the $\triangle 52$ BAC, diluted out the plasmid ORF52 as well.

In my attempts to effectively use this BAC, I altered multiple conditions. I transfected plasmid ORF52 into cells prior to transfection of BAC, at the same time, 24- or 48 hours after BAC DNA transfection, or throughout the $10+$ BAC transfection period. I varied the amounts of plasmid as well as BAC DNA. I attempted different transfection reagents, and attempted to use HEK293 cells, which are more amenable to transfection. Regardless of numerous changes to each condition, I never saw evidence of CPE or virus production (Figure A1-4). In addition to RhF cells, I also did a time-course using HEK293 cells, because they are easier to transfect than RhF. Upon Transfection of HEK293 cells with ORF52 plasmid (pk-mycORF52), $\Delta 52$ BAC, ORF52 plasmid $+\Delta 52$ BAC, or wt BAC, I was only able to detect ORF52 in cells transfected with the ORF52 plasmid and not in any other condition, including wt BAC. Myc-tagged ORF52 expression was robust at 5 days post-transfection, had significantly decreased at 7 days, and was gone at 13 days (Figure A1-5). I had already shown that in RhF cells the wt BAC did produce virus, which led us to believe that HEK293 cells did not support RRV lytic replication and would not be useful for our experiments. 
Figure A1-4. Examination of viral proteins expressed in wt BAC and $\Delta 52$ BAC transfected RhF cells. A) Western blot of cell lysates to examine expression of viral proteins including MCP, SCIP, and ORF52 in RhFs infected for 48 hours as a positive control (+) (lane 1), uninfected RhFs as a negative (-) control (lane 2), RhFs transfected with a wt BAC for 11 days (lane 3), or $\Delta 52$ BAC (lane 4). B) Concentrated supernatants collected from the BAC transfected cells in A were probed for the viral proteins MCP, SCIP, and ORF52. Wt BAC supernatant (lane 1) and $\triangle 52 \mathrm{BAC}$ (lane 2) 

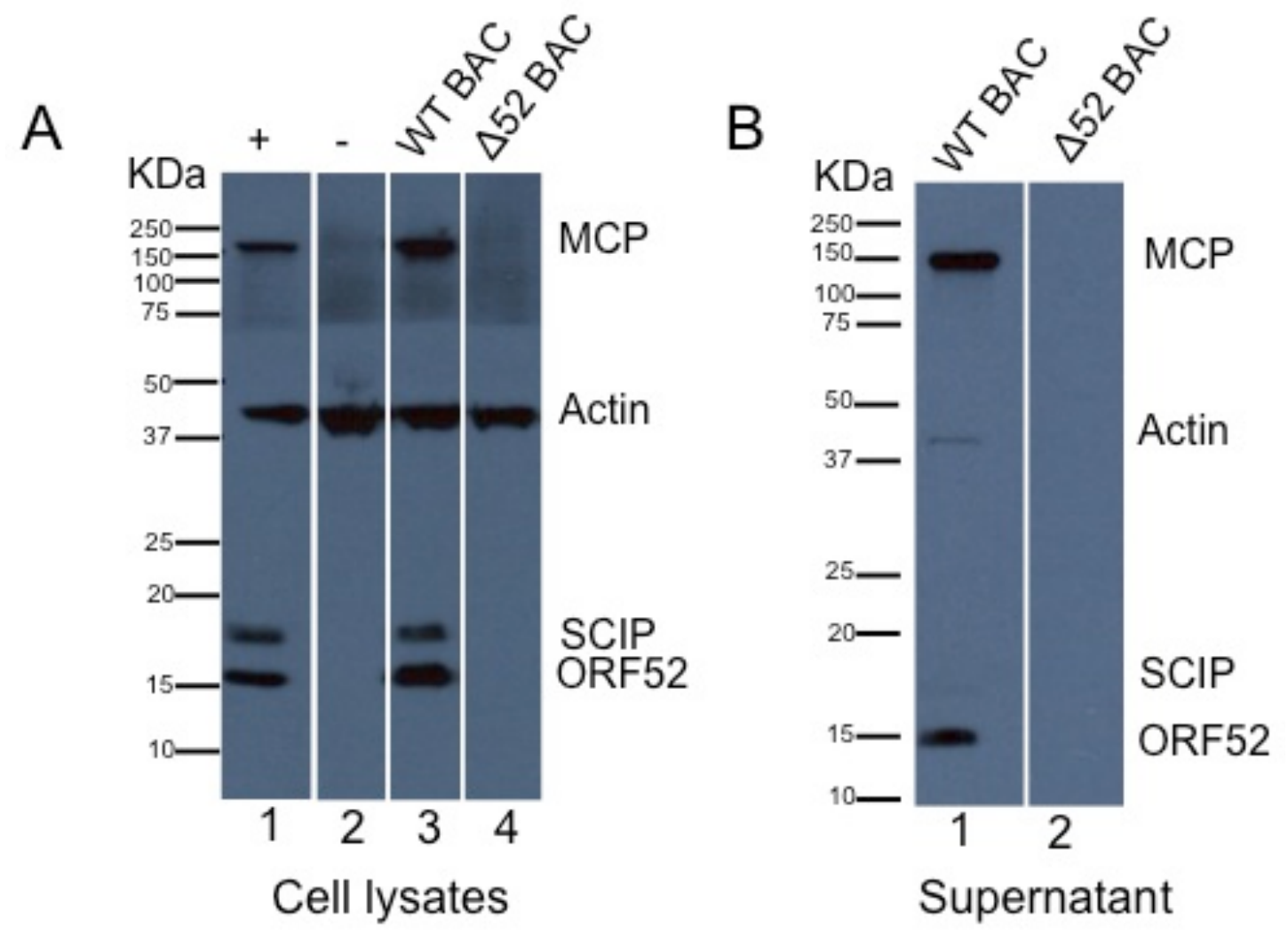
Figure A1-5. Time course of ORF52 protein expression in HEK293 cells transfected with wt BAC or $\triangle 52$ BAC. HEK293 cells were transfected with ORF52 plasmid (pk-mycORF52) only (lane 2), $\Delta 52$ BAC only (lane 3), ORF52 plasmid and $\triangle 52 \mathrm{BAC}$ (lane 4), or wt BAC (lane 5) and probed for ORF52, MCP, or cellular actin on days $5(A), 7(B)$, or $13(C)$. RRV infected RhFs are used as a positive control (+) (lane 1) in A, B, and C. myc-tagged ORF52 is robustly expressed at 5 days post plasmid transfection (lanes $2 \& 4$ in A). That expression is significantly less 7 days posttransfection (B) and gone at day $13(\mathrm{C})$. There is no wt virion produced (non-tagged) ORF52 in any lanes except control at any time point $(A, B$, or $C)$. 


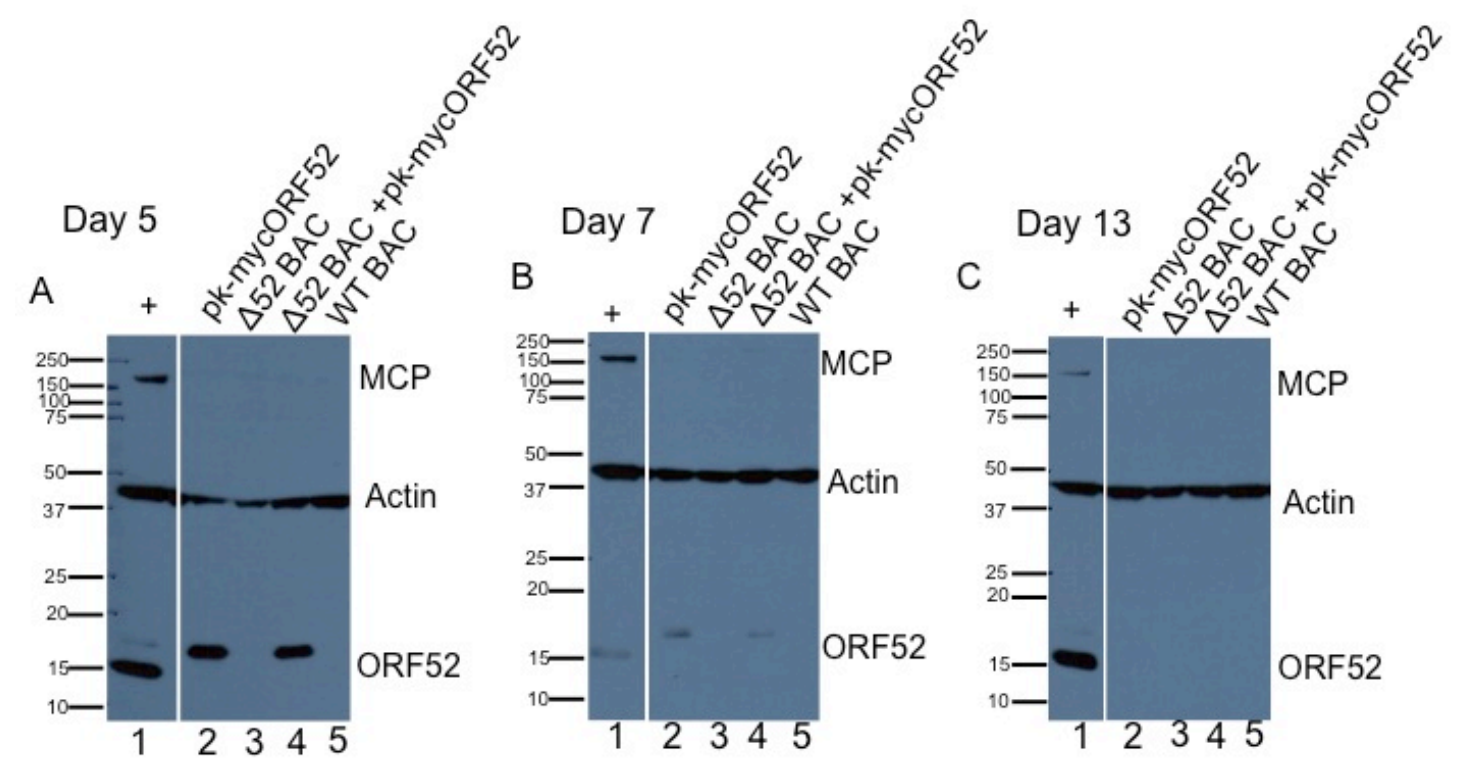


APPENDIX 2: shORF52 AND shSCRAMBLED EXPRESSING RHF CELL LINES 
We decided to utilize a shRNA lentiviral vector approach to try and circumvent the low transfection efficiency of RhF cells. Utilizing this system, all (or almost all) cells would express shRNA targeted to ORF52 (shORF52), or a scrambled sequence (shScrambled) (control), in RhF cells, which we could select with hygromyocin. Following development of a stable population of RhF expressing shORF52 or an shScrambled, we would subsequently infect these cells with wt RRV and go on to investigate the role of ORF52 in cells expressing ORF52 (shScrambled + RRV) or with ORF52 knocked down (shORF52 + RRV) as done in Chapter 2.

In general, to generate lentivirus, plasmids for the transgene expression cassette, packaging and envelope vectors are co-transfected into permissible cell line, such as 293T cells. Produced lentivirus is then used to transduce the desired target cell. Only the vector containing the shRNA expression cassette integrates into the host genome in transduced cells. The shRNA hairpin in the expression cassette is cleaved by the cellular machinery into siRNA, which then binds to the RISC complex, leading to matching of siRNA bound to it to complementary mRNA sequences, and subsequently cleaves them so they are unable to be translated (Figure A2-1) (reviewed in $(91,191,312)$ ).

To create the lentivirus, we used the pLKO.1 hygromyocin vector (Addgene) and their protocol (Figure A2-2). pLKO.1 hygro lentiviral vector carries a hygromycin resistance cassette for selection in transfected cells. An Agel and an EcoRI restriction site flank the cloning site (labeled 'shRNA' in Figure A2-2) used for insertion of the shRNA construct containing the siRNA sequence of 
Figure A2-1. Overview of lentiviral creation and shRNA processing. Image broadly outlines the three different vectors necessary for generation of lentivirus, transduction into target cell, processing of shRNA, and destruction of mRNA. Image adapted from (191) and used with permission. 


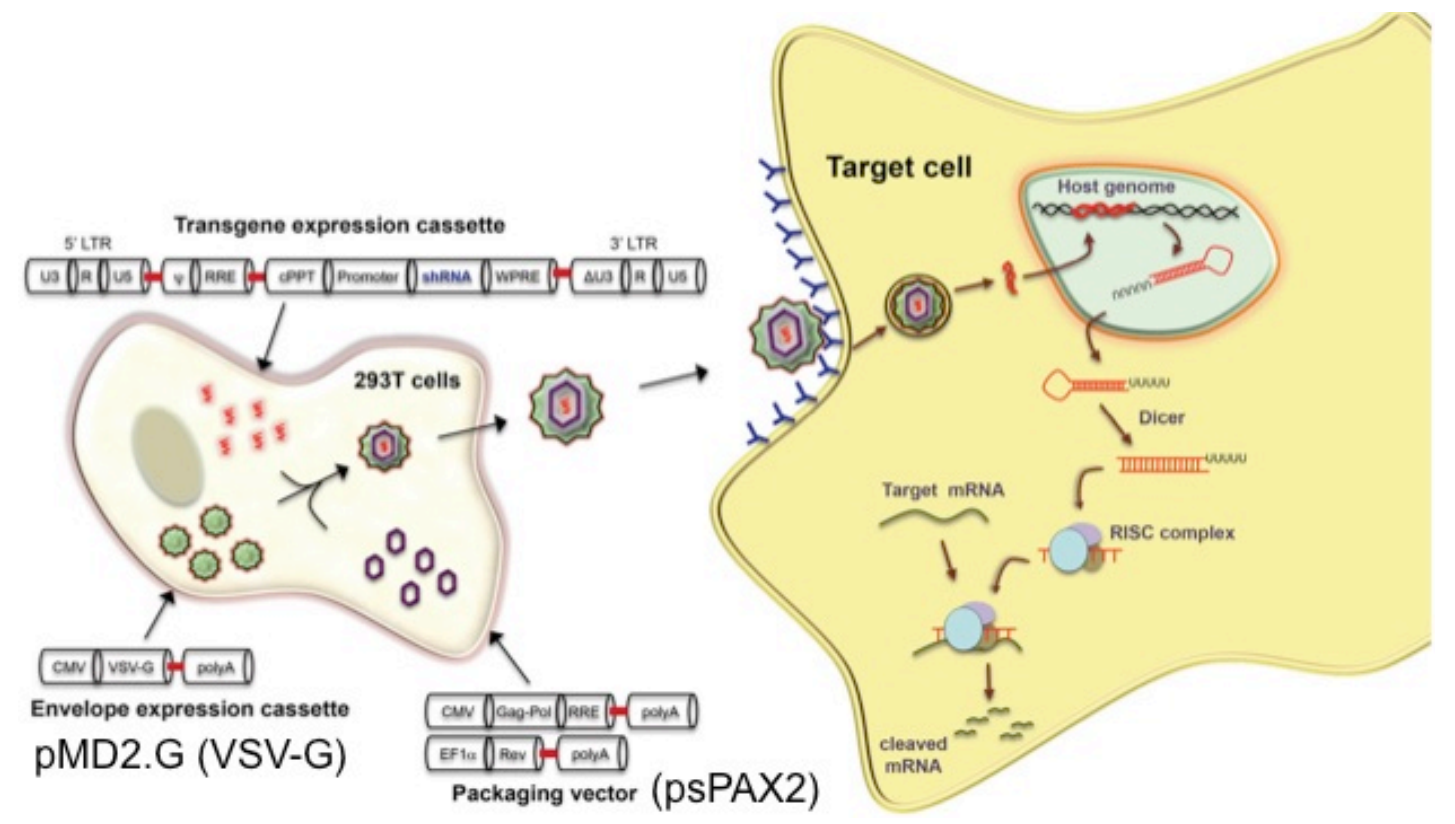


Figure A2-2. Map of lentiviral expression vector pLKO hygro. pLKO hygro is driven by the $U 6$ promoter, has an Amp resistance gene for prokaryotic selection, and a hygromyocin resistance gene for eukaryotic selection. Image adapted from Addgene, an open source website (http://www.addgene.org/tools/protocols/plko/ and accessed 4/24/14). 


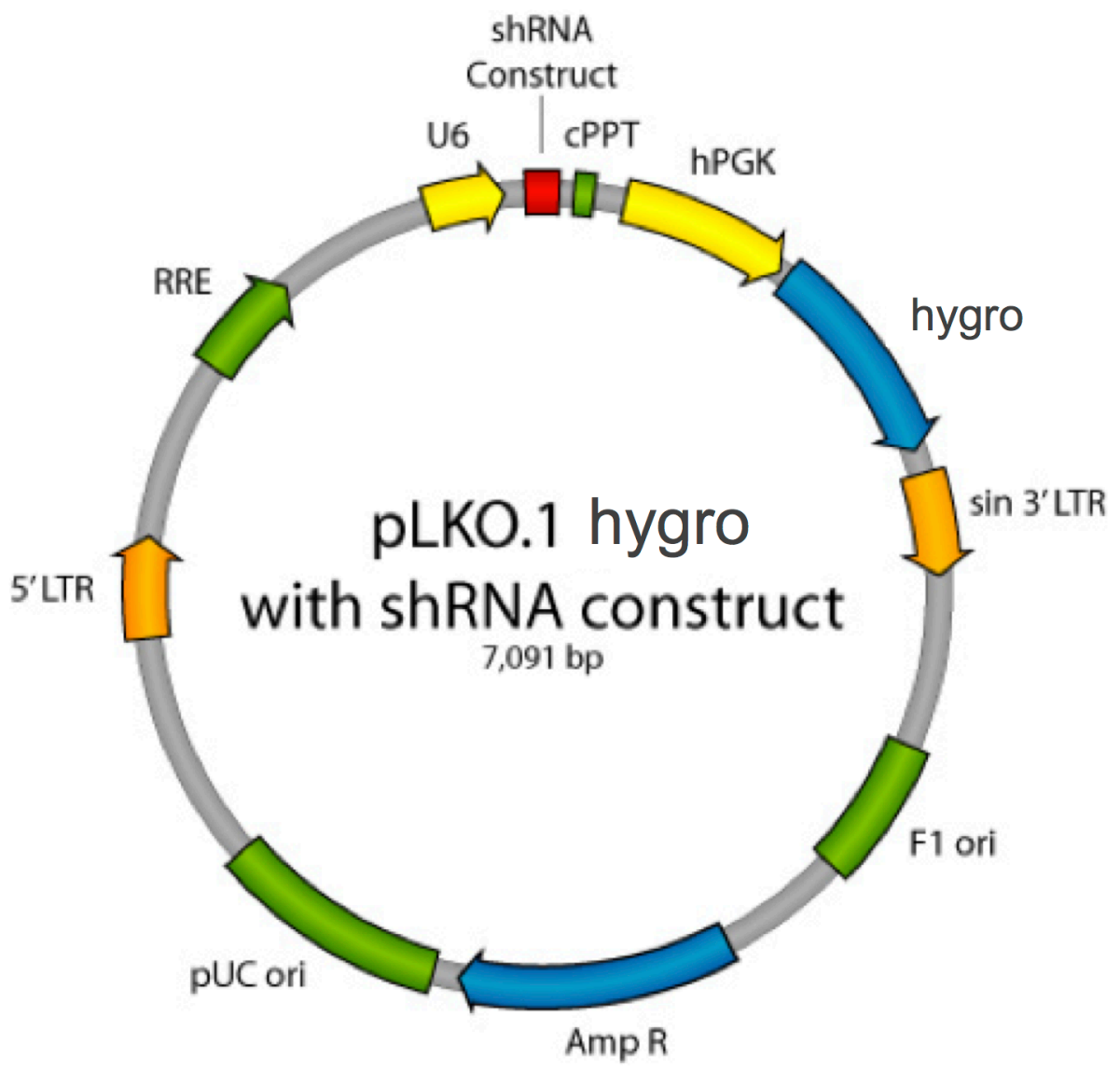


choice. Additionally, the shRNA has a 6-nucleotide sequence recognized by Xhol, the siRNA anti-sense siRNA sequence, and a termination sequence (Figure A2-3). To create the shRNA construct, we entered our siRNA sense and antisense sequences into the aforementioned format and ordered corresponding oligosaccharides (oligos).

We then cut the pLKO.1 hygro vector sequentially with Agel and EcoR1 and combined the cut vector with annealed shRNA construct oligos at different oligo:plasmid (ligation mix) ratios $(1: 1,4: 1,2: 2)$. We then transformed Stbl2 $E$. coli with $5 \mu \mathrm{L}$ of ligation mix and following clonal selection, maxi prep, and sequence confirmation, had our shRNA constructs containing both shORF52-3 and shScrambled. The shScrambled construct contained the same siORF52 nucleotides; they were just scrambled in a random order to use as a negative control.

Next we generated lentivirus expressing our shRNAs by co-transfected $293 \mathrm{~T}$ cells with pLKO.1 hygro vector, a packaging vector (psPAX2), and an envelope vector (pMD2.G) (gifts of Bouton laboratory). We collected supernatants from transfected 293Ts at 24,48 , and 72 hours post transfection, combined them, and filtered them through a $0.45 \mu \mathrm{M}$ filter. We then added $1 \mathrm{~mL}$ of supernatant containing lentivirus to naïve RhF cells. 48 hours later hygromyocin was added to select for transduced cells. Selection of transduced RhF cells continued with replenishment of media containing hyrgomyocin until a pool of stably transduced RhF cells were generated. We went on to test the 
Figure A2-3. Diagram of shRNA construct including shORF52 and shScrambled oligosaccharide design sequences. A shRNA construct was designed to target RRV ORF52 and a negative control shShrambled construct composed of the same RRV ORF52 amino acids, just scrambled and nontargeting. Image from Addgene, an open source website (http://www.addgene.org/tools/protocols/plko/ and accessed 4/24/14). 


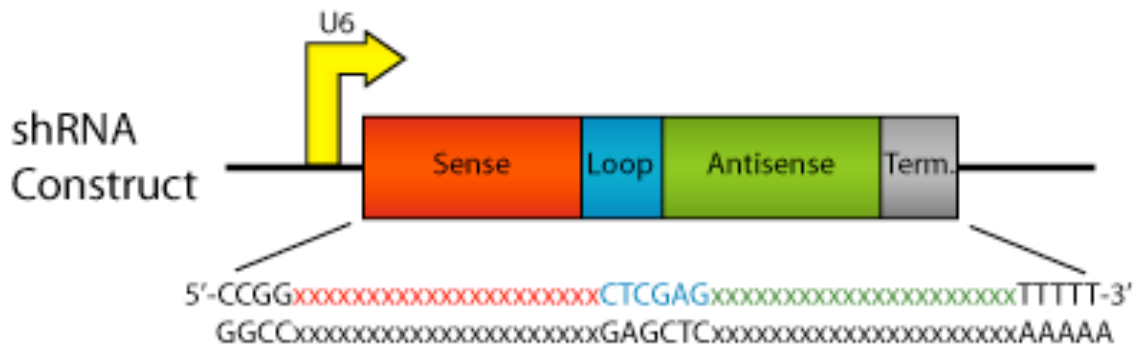

ShRNA

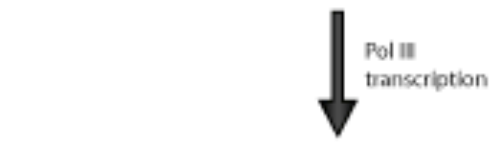

CCGG: Agel restriction enzyme site

AATTC: EcoRI restriction enzyme site

TTTTTG: polyT site for RNA polmerase III

CTCGAG: loop contains Xhol cleavage site

siORF52 sense: 5' CCCGTAAGATTGAAGCTAATT 3'

siORF52 antisense: 5' AATTAGCTTCAATCTTACGGG 3'

siScrambled sense: 5' CATTACGTTGTAAGCTAGACA 3'

siScrambled antisense: 5' TGTCTAGCTTACAACGTAATG 3'

shORF52-3 Fwd construct:

5' CCGGCCCGTAAGATTGAAGCTAATTCTCGAGAATTAGCTTCAATCTTACGGGTTTTTG 3'

shORF52-3 Rev construct

5’ AATTCAAAAACCCGTAAGATTGAAGCTAATTCTCGAGAATTAGCTTCAATCTTACGGG 3’

shScrambled construct:

Fwd oligo

5' CCGgCATTACGTTGTAAGCTAGACACTCGAGTGTCTAGCTTACAACGTAATGTTTTTG 3'

Rev Oligo

5' AATTCAAAAACATTACGTTGTAAGCTAGACACTCGAGTGTCTAGCTTACAACGTAATG 3' 
efficacy of these cells following transfection with exogenous ORF52 and probing the cell lysates for ORF52 expression. Initially use of these cell lines seemed promising, as there was a decrease in ORF52 in the shORF52 expressing cells compared to shScrambled (Figure A2-4, panel A); however these cells lost their ability to knockdown ORF52 when cultured over time (Figure A2-4, panel B). Additionally, this knockdown ability was lost following freeze/thaw of early cell passages.

Rather than taking a great deal of time to troubleshoot this situation, or transducing naïve RhFs with shORF52 lentivirus for each new experiment, which may not have worked, we decided to move on and back into transient siRNA transfection to investigate the role of RRV ORF52. This made particular sense given the time already spent on the BAC. 
Figure A2-4. sh52 knocks down expression of transfected pk-mycORF52; however, knockdown is not stable. RhF cell lines stably expressing shORF52 (sh52) or shScrambled (shScram). Cells were transfected with $1 \mu \mathrm{g}$ of pk-myc ORF52 plasmid DNA per $1 \times 10^{6}$ cells and harvested 48 hours later. Equal amounts of protein were run and western blots were probed with anti-ORF52 and anti-actin. 


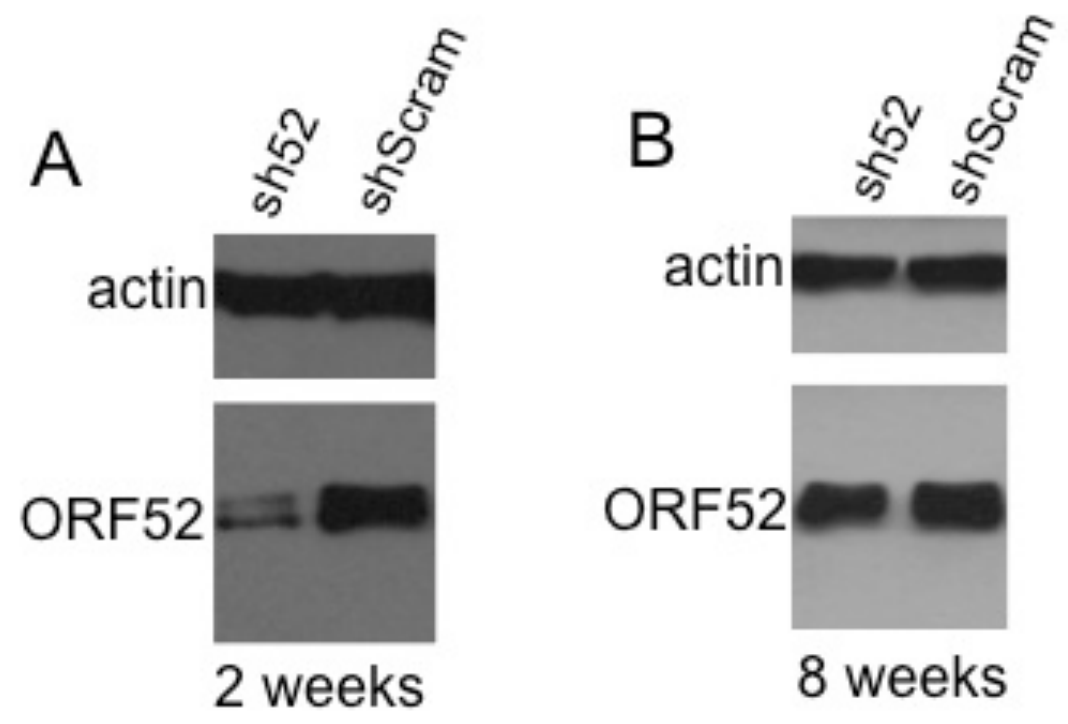


APPENDIX 3: TET-INDUCIBLE ORF52 AND SIORF52 RESISTANT RHF CELL LINES 
In our continued pursuit to determine RRV ORF52 function, we decided to move away from the BAC and instead utilize siRNA-mediated knockdown of ORF52 during wt RRV infection. In order to confirm the role of ORF52 we knew we would need to show rescue of phenotypes linked to ORF52 knock down by providing ORF52 in the system. RhF cells are transfected with plasmid DNA at a low efficiency ( $40 \%$ in best case scenarios with Amaxa) and we hoped to increase this efficiency by creating inducible wt ORF52 and siORF52 resistant (Res52) RhF cell lines. Our plan was to transfect these inducible RhF cells with siRNA to ORF52 or control, then infect them with RRV, and at some point induce expression of Res52, with the hope that it would be available and able to complement the ORF52 knockdown. For this purpose, we created tet-inducible ORF52 RhF cell lines using the Flp-In T-Rex kit (Life Technologies). This system allows the creation of a mammalian host cell line that contains an integrated Flp recombination target site (FRT) and stably expresses the tetracycline (tet) repressor (tetR). One can then able to utilize this cell line and express any cloned gene of interest under a tet-inducible system.

We first transfected linearized $\mathrm{pFRT/lacZeo} \mathrm{plasmid} \mathrm{into} \mathrm{RhF} \mathrm{cell} \mathrm{via}$ Amaxa transfection (Lonza). The pFRT/lacZeo vector contains a lacZ-Zeocin fusion and a FRT site. Following transfection, we selected for RhF cells with the integration with Zeocin. We isolated nine individual clones and expanded them. Then, using Southern blot, we screened these clones to identify clones with a single FRT integration site using a lacZ probe. We first determined the concentration of lacZ probe by dot blot and comparison to a known standard 
Figure A3-1. Establishment of a stably transfected pFRT/LacZeo RhF host cell line. (A) Dot blot of LacZeo probe used to determine concentrion for Southern blot. (B) DNA isolated from pFRT/LacZeo transfected RhF and amplified by PCR using LacZ primers, (C) Agarose gel of DNA isolated from pFRT/LacZeo transfected clones and cut with HindIII and (D) Southern blot from Agarose gel separation. (See text for more detailed explanation.) 

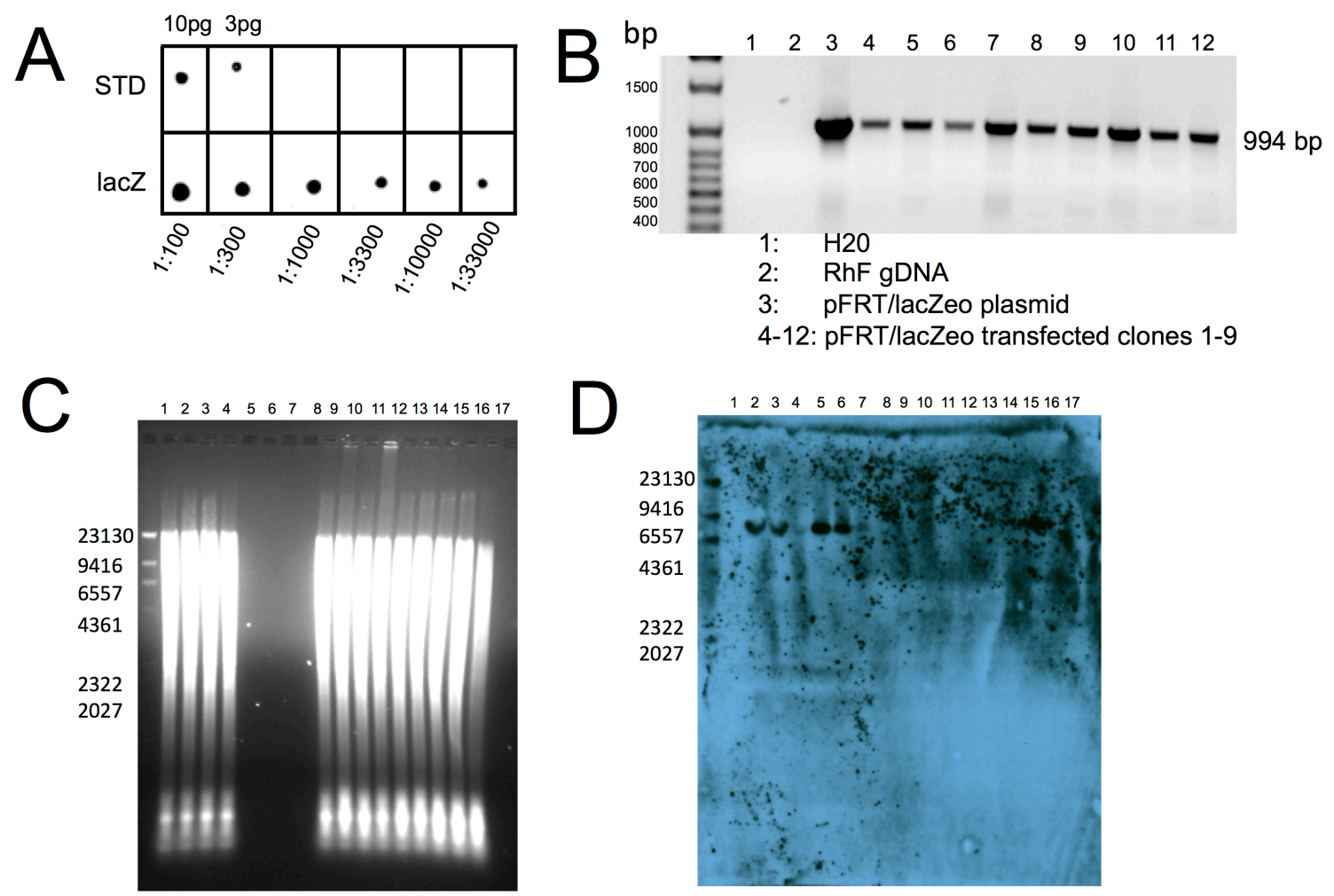

(C) and (D) gel order

1: UNTF RhF

2: $\quad$ UNTF RhF + pFRT/lacZeo plasmid

5-8: pFRT/lacZeo plasmid

9-17: pFRT/lacZeo transfected clones 1-9 
(Figure A3-1A). Finally, we ran an agarose gel of DNA isolated from our clones (Figure A3-1C) and performed the Southern blot using the lacZ probe (Figure A3-1D). Unfortunately, we experienced technical issues with the Southern blot and after several months were still unable to resolve them and detect lacZ in any of our clones. To ensure that these clones were actually integrated with pFRT/lacZeo, we performed PCR of each of the 9 clones using LacZ primers (Figure A3-1B). Since each clone had DNA amplified with the LacZ primers, meaning integrated sequence, we decided to continue on with the protocol and cell line development without confirmation of single integration.

Stable clones 1-9 that were resistant to Zeocin were next transfected using Amaxa with the pcDNA6/TR plasmid that contains the tetR gene and conferred Blasticidin resistance. Following selection of clones resistant to both Zeocin and Blasticidin we tested for $\beta$-galactosidase activity (Figure A3-2). The protocol recommends selecting the clone with the greatest $\beta$-galactosidase activity to use for the subsequent steps. We selected clone 4 , which had the highest expression, as well as clone 7 that had moderate $\beta$-galactosidase activity. We found clone 4 to be most effective and is the clone used in this section.

We co-transfected clones via Amaxa with linearized pcDNA5/FRT/TO expression vector that contained our gene of interest, ORF52, and pOG44. Next, we cloned ORF52 into pcDNA5/FRT/TO, a vector that contained a tetON gene and a hygromycin resistance gene. Plasmid pOG44 expressed the FLP 
Figure A3-2 $\beta$-galactosidase activity in RhF cells with $\mathrm{pFRT/lacZeo}$ integration. $\beta$-galactosidase activity was measured by combining cell lysates with ONPG and measuring hydrolysis of ONPG to ONP anion at an absorbance of $420 \mathrm{~nm}$. 


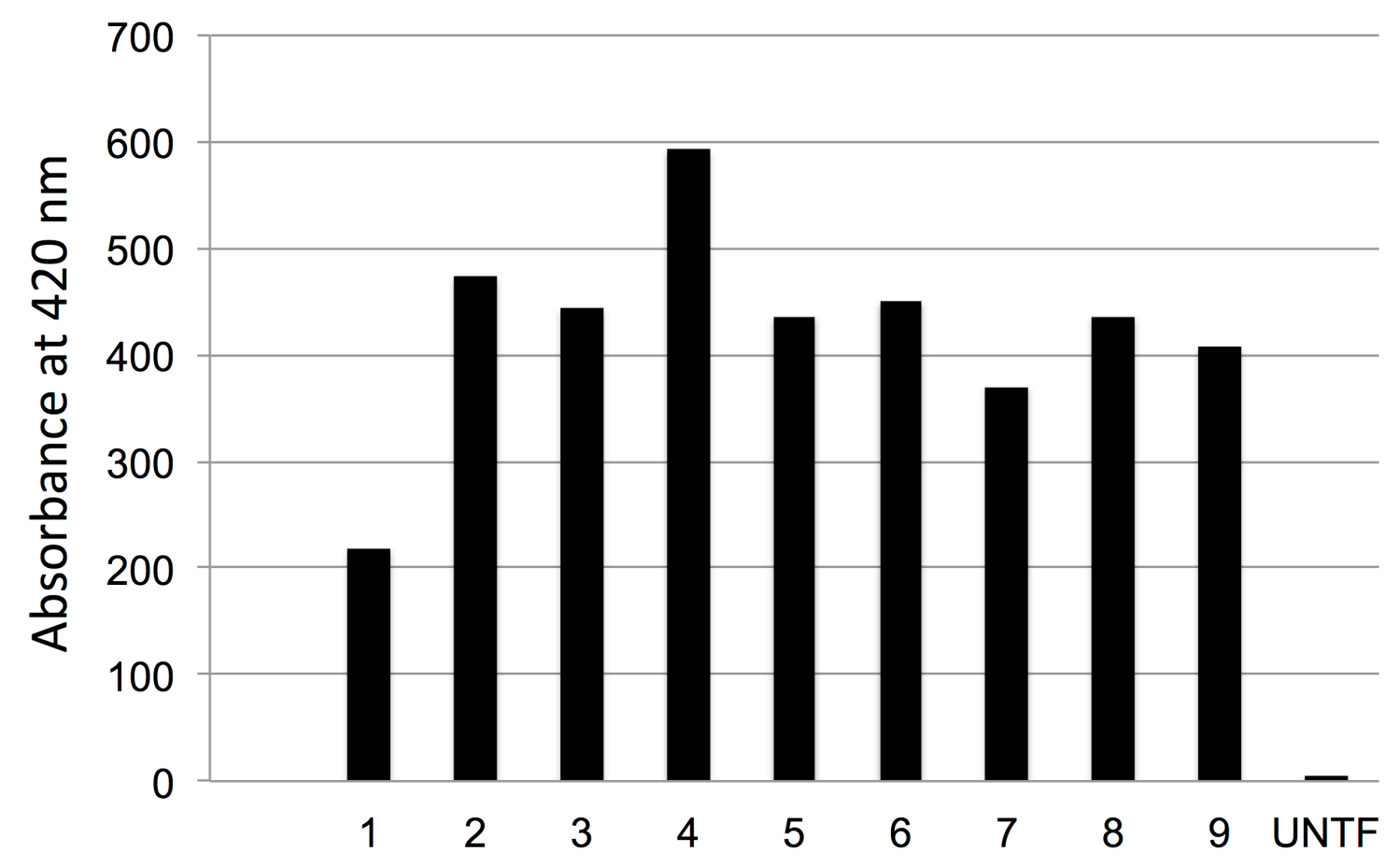


recombinase gene that, when co-transfected, allowed for integration of ORF52-pcDNA5/FRT/TO into the established FRT site in the RhF host cell line.

We then selected for clones of ORF52 integrated RhFs with Hygromycin and Blasticidin. Following expansion of cells, we tested individual clones for both uninduced (leakiness) expression of ORF52, as well as expression of ORF52 following doxycycline (a tetracycline antibiotic) addition. Several of the clones tested negative for ORF52 expression when uninduced $(U)$ and positive when induced (I) (Figure A3-3).

At this time, we planned to further our ORF52 knockdown studies by transfecting into our inducible cell line a siRNA targeted to ORF52. Tests of several sequences revealed two that gave the best knockdown of ORF52 with the most minimal side effects labeled as ' $c$ ' and ' 3 '. Initially, when we made this tet-inducible ORF52 RhF cell line, we were working with siORF52c sense strand with the sequence 5'- GCUGAUAAAUUCUGGGGAUUU. However, in order to assign a specific function of ORF52, we needed to be able to rescue the phenotypes with the knockdown. Instead of trying to complement by transiently transfecting ORF52 into these cells, we created a tet-inducible siRNA resistant ORF52 RhF cell line, using the already established FRT site integreated RhF host cell line. We made a mutation to 2 wobble bases in ORF52 that were in the area targeted by the siRNA, which we hoped would confer siRNA resistance. Then we created a siResORF52c forward primer changing the TCT to TCC and GGG to GGA (changed bases are underlined) 5' AAAAGCTGATAAATTCCGGAGATCCCACCAGGTCCGG. The bolded area is 
Figure A3-3. Tet-inducible RhF clones express ORF52 upon induction by doxycycline. Western blot of several different clones uninduced $(U)$ or induced (I) with doxycycline and probed with anti-ORF52 or anti-actin. 


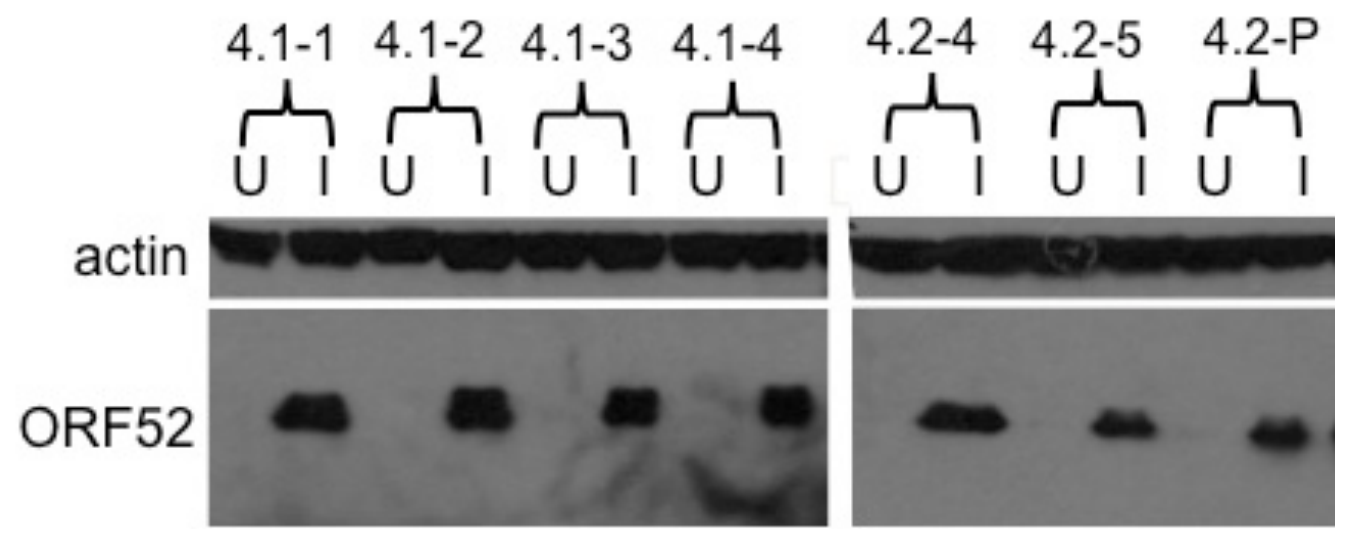


the siRNA target sequence. We then performed site directed mutagenesis (Stratagene) using the already created ORF52-pcDNA5/FRT/TO plasmid and the oligos with the desired mutation following the Stratagene protocol. Afterward, we then co-transfected this siResistantORF52c-pcDNA5/FRT/TO and pOG44 and selected for integrated clones as described previously.

Tests performed on one of our clones for uninduced ORF52 expression and expression following 48 hours induction with doxycycline and the yielded promising results (Figure A3-4).

We next went on to test whether this induced Res52c was resistant to siORF52c. We transfected these cells with siORF52c and induced with Dox 24 hours later. 48 hours following induction, we collected cells and saw that Res52 was resistant to knockdown with siORF52c (Figure A3-5).

In subsequent experiments we used these Res52c RhF cells in RRV infection experiments. After transfection with siRNA, cells were infected with RRV at an $\mathrm{MOI}$ 5. After removing the input virus, we replenished media containing 40 $\mathrm{ng} / \mathrm{mL}$ or $1 \mu \mathrm{g} / \mathrm{mL}$ Dox (Figure A3-6).

In all conditions at both $72-$ and $96-\mathrm{h}$ p.i. in the cell lysates, we saw expression of MCP, ORF45, and SCIP. ORF52 was expressed in the siCNL treated cells as well as in the cells induced with the Res52 (si52c $+40 \mathrm{ng} / \mathrm{mL}$ or si52c $+1 \mu \mathrm{g} / \mathrm{mL}$ Dox) (Figure A3-6A lanes 1, 3, 4, 6, and 7), but not in cells with ORF52 knockdown and no rescue (si52c) (Figure A3-6A lanes 2 and 5). Supernatants collected from these cells and concentrated show that MCP, ORF45, SCIP, and ORF52 are packaged in particles in siCNL (Figure A3-6A, 
Figure A3-4. Tet-inducible siResistant ORF52c RhF cells express ORF52 following induction with Dox. RhF cells with integrated Res52 express ORF52 upon induction (lane 2) with Dox. Western blot of cell lysates probed with antiORF52 and anti-actin. 


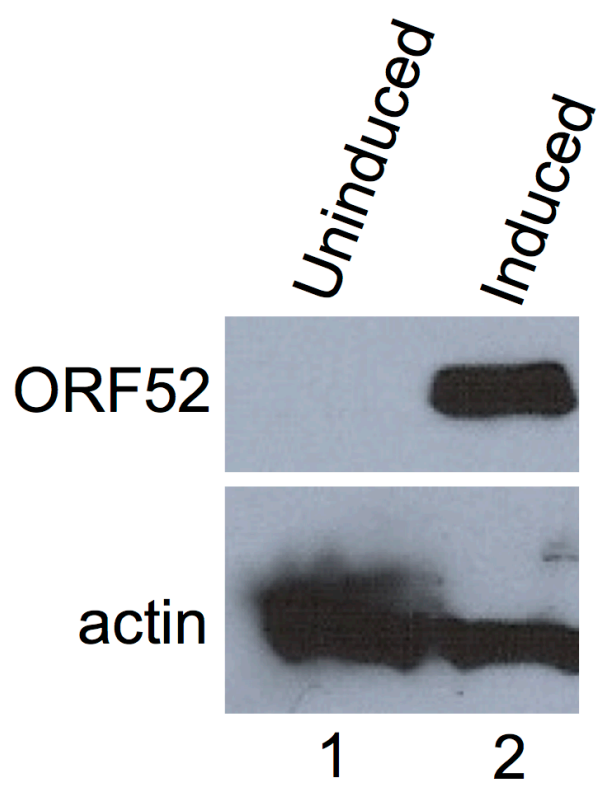


Figure A3-5. Tet-inducible siResistant ORF52c RhF cells express ORF52 upon addition of Dox and it is resistant to knockdown by siORF52c. Res52 is resistant to knockdown by siORF52. Cells were transfected with siRNA and induced with $1 \mu \mathrm{g} / \mu \mathrm{L}$ of Dox for 48 hours. Western blot of cell lysates probed with anti-actin and anti-ORF52. 


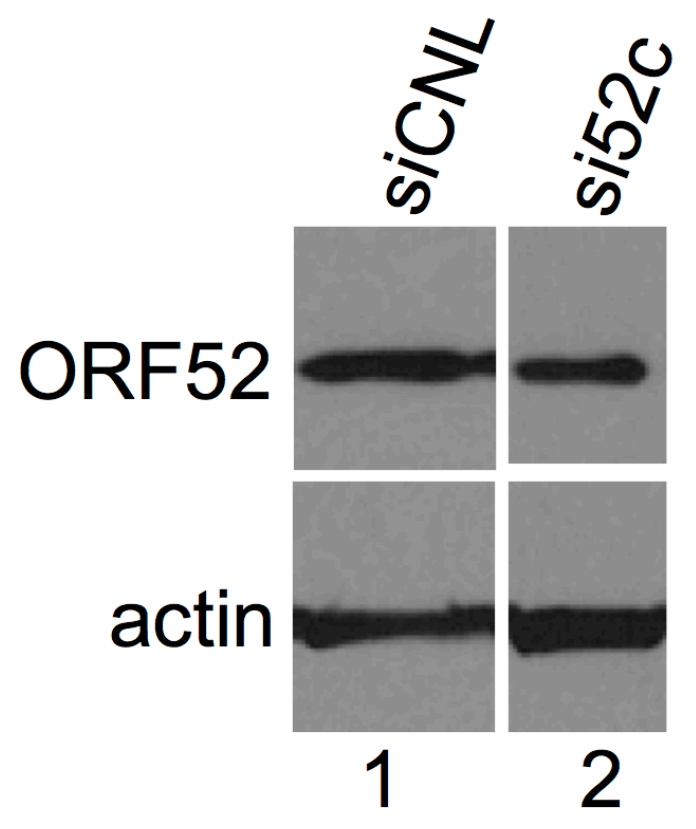


Figure A3-6. Expression of viral proteins in RhF cells and in released particle following ORF52 knockdown and rescue. Tet-inducible Res52c RhF cells were transfected with siORF52c for 24 hours. Cells were infected with wt RRV at an $\mathrm{MOI}$ of 5 for 1 hour. Following removal of input virus, cells were washed with PBS and media containing no Dox (siCNL and si52c) or rescue (si52c $+40 \mathrm{ng} / \mathrm{mL}$ or si52c $+1 \mu \mathrm{g} / \mathrm{mL}$ Dox), $40 \mathrm{ng} / \mathrm{mL}$ or $1 \mu \mathrm{g} / \mathrm{mL}$ Dox was added. (A) 72 or 96 hours later cells were harvested and cell lysates probed for MCP, ORF45, SCIP, ORF52, and actin. (B) Supernatants were collected and layered over a $35 \%$ sucrose cushion. Following concentration in a centrifuge at $65,204 \times \mathrm{g}$ for 30 minutes at $4^{\circ}$ samples were resuspended in lysis buffer, boiled for 10 minutes, and equal volumes were run. 

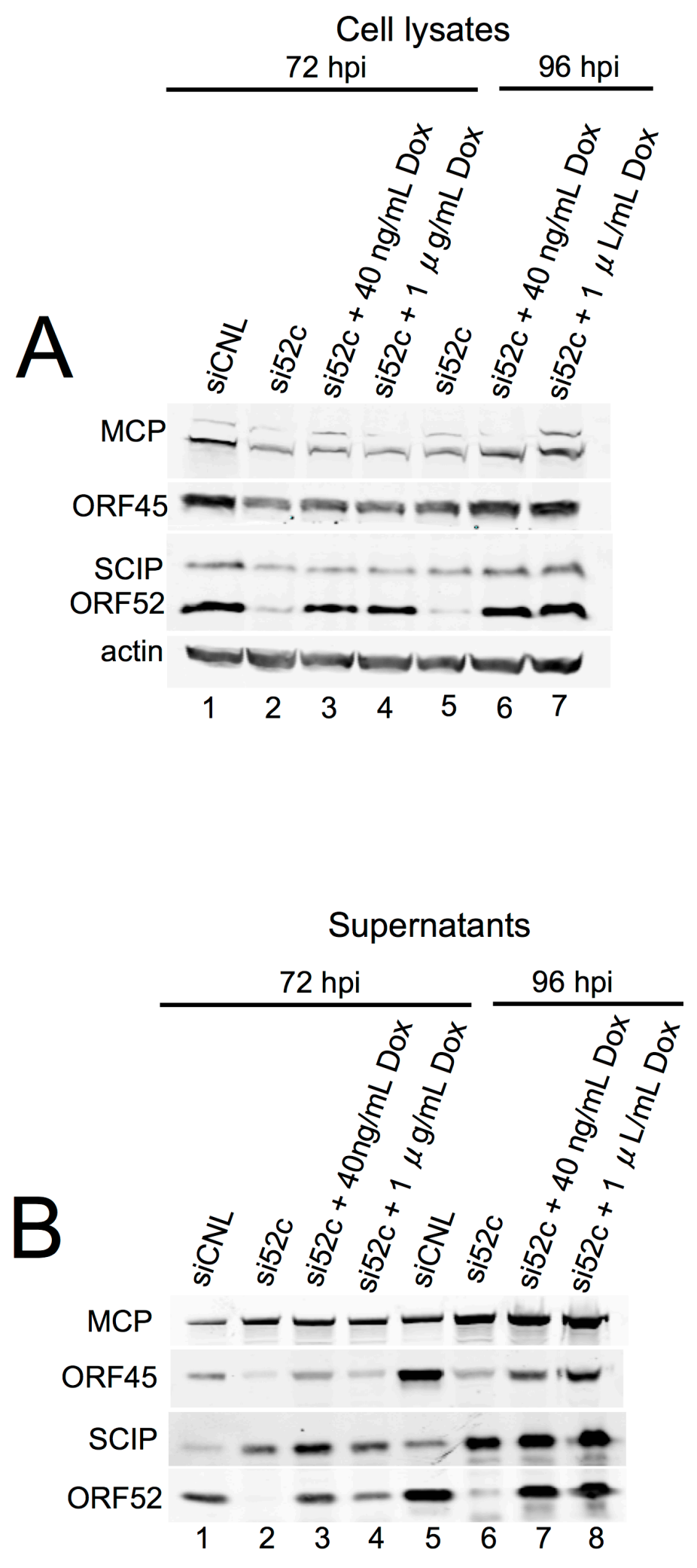
lanes 1 and 5). In si52 conditions, with no Dox, at both time points, there is minimal packaging of ORF52 and ORF45 in the viral particle compared to siCNL (Figure A3-6, lanes 2 and 6). When cells are induced with $40 \mathrm{ng} / \mathrm{mL}$ or $1 \mu \mathrm{g} / \mathrm{mL}$ Dox and at 72 and $96 \mathrm{~h} \mathrm{p.i.} \mathrm{there} \mathrm{was} \mathrm{a} \mathrm{partial} \mathrm{rescue} \mathrm{of} \mathrm{ORF52} \mathrm{and} \mathrm{ORF45}$ packaging, indicated by an increase in expression over si52 (Figure A3-6B, lanes $3,4,7$, and 8 ).

Next, we measured the titer in tet-inducible Res52c RhF cells at 48,72 , and 96 hours post infection. Based on our western blots that showed expression or ORF52 in the cell lysates following induction (Figure A3-6A, lanes 3, 4, 6, and 7) and the increase in packaging on ORF52 and ORF45 in the particle of Dox induces RhF cells (Figure A3-6B, lanes 3, 4, 6, and 7) we expected to see a robust increase in viral titer of Dox induced (rescue) over si52 with no Dox (kd). In siORF52c treated cells, titers decreased 35 fold at 48 h p.i., 200 -fold at $72 \mathrm{~h}$ p.i., and 168 -fold at $96 \mathrm{~h} \mathrm{p.i.} \mathrm{from} \mathrm{siCNL.} \mathrm{While} \mathrm{induction} \mathrm{of} \mathrm{Res52} \mathrm{was} \mathrm{able} \mathrm{to}$ partially rescue this decrease, it was to a very minimal extent of 2-, 3, or 6- fold, respectively, depending on time point (Figure A3-7).

We also established a second tet-inducible siResistant ORF52 RhF cell line that was resistant to a different siORF52 siRNA, siORF52\#3 (the siORF52 sequence used in Chapter 2), and while several clones did express ORF52 following Dox addition, we did not pursue their use for further studies. This was primarily because, in addition to the minimal rescue in viral titer with this cell line, we observed these Tet-inducible RhFs (both wt ORF52 and Res52) did not behave and look exactly like non-manipulated RhF cells. First of all, during wt 
Figure A3-7. Titers of siCNL, ORF52 knockdown, or ORF52 rescue determined by plaque assay 48-, 72-, or 96 hpi. Res52 tet-inducible RhF cells were treated with siCNL or siORF52. 24 hours later cells were infected with RRV at an $\mathrm{MOI}$ of 5 and induced for Res52 expression with Dox. At the indicated time points, media was collected and titers determined by plaque assay. 


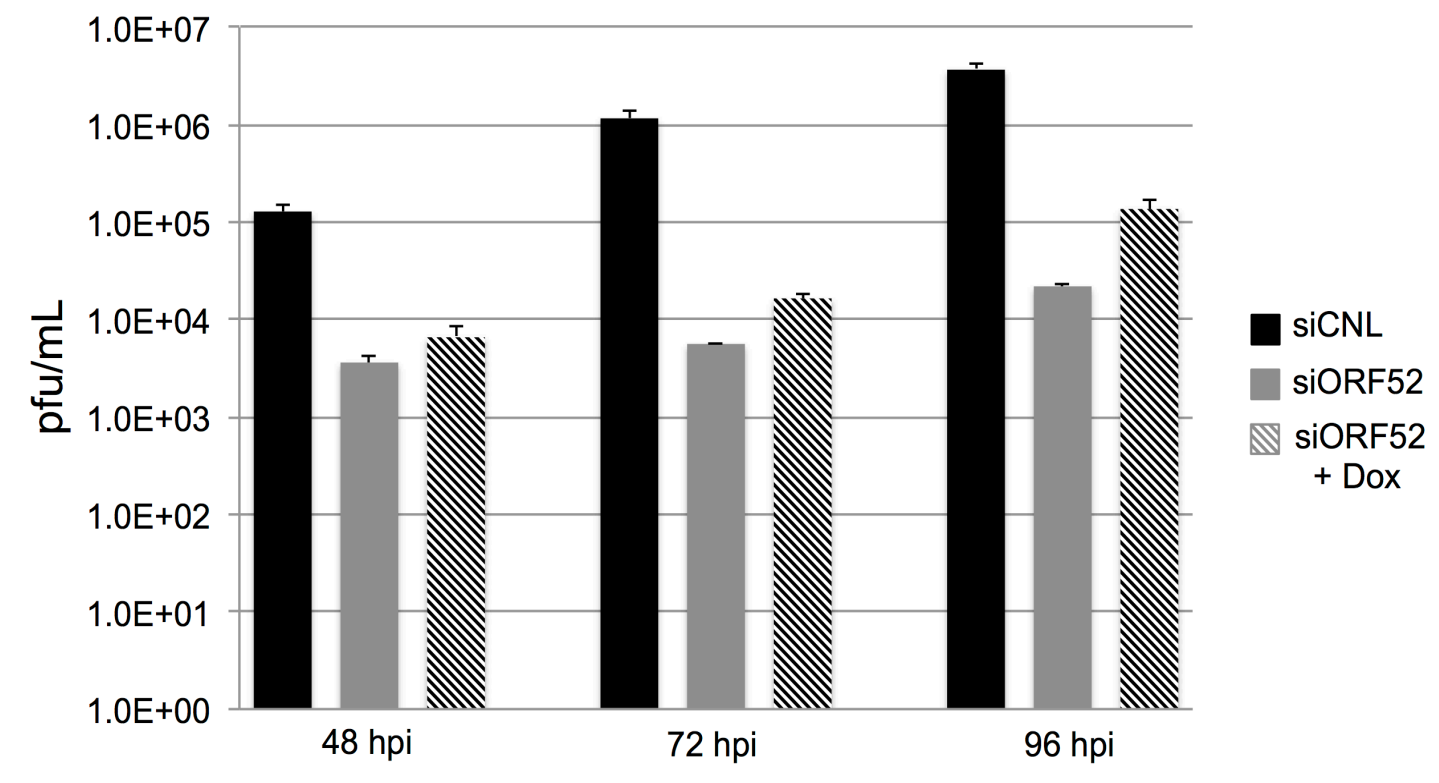


RRV infection, the infection progressed at a slower rate, approximately 48 hours slower than wt RRV. For example, at $48 \mathrm{~h}$ p.i. in siCNL treated siResORF52 RhF, the tilter was $1.29 \times 10^{5} \mathrm{pfu} / \mathrm{mL}$. In wt RRV infected RhFs the titer is $7 \times 10^{6} \mathrm{pfu} / \mathrm{mL}$ on average - over 50 -fold difference - and it never catches up to wt RRV titer. Even $96 \mathrm{~h}$ p.i. in the tet-inducible siResistant ORF52 RhF cells the titer is still half of that in wt RRV infected RhFs $\left(3 \times 10^{6} \mathrm{pfu} / \mathrm{mL}\right)$ in contrast to wt RRV infection, where there are no cells left at $72 \mathrm{~h}$ p.i. because they have all lysed from infection. Additionally, the cells appeared to have more nucleoli regions in the nucleus; the relevance, or lack thereof, is unknown. 
APPENDIX 4: ORF45 AND ITS ROLE IN THE SUSTAINED ACTIVATION OF ERK IN RRV 
Previous work in our laboratory by Evonne N. Woodson investigated the roles of extracellular signal-regulated kinase 1 (ERK1) and ERK2 in the structure and function of RRV (341). She found that RRV infection activated the ERK pathway, that activated (phosphorylated) ERK2 (pERK2) was preferentially incorporated into mature virions in the tegument layer, and that the activation of ERK was required for production of viral proteins and virions (341).

ORF45 is a gammaherpesvirus specific tegument protein packaged into mature KSHV and RRV virions $(239,277,358)$. In KSHV, ORF45 induces sustained ERK and p90 ribosomal S6 kinase (RSK) activation during KSHV lytic reactivation $(169,170)$. It was suggested that ORF45 formed a heterotrimeric complex providing protection from phosphatases that would otherwise dephosphorylate/inactivate ERK and RSK (170).

As part of continued research in our laboratory, a second paper investigating ORF45 and its role in the sustained activation of ERK in RRV was recently published in PLoS Pathogen (340). In this study, we demonstrated that in RRV pERK levels continued to rise throughout the lytic cycle and that RRV ORF45 interacts with both ERK and RSK in infected cells forming heterotrimeric complexes within the nucleus. Also, RRV infection led to the activation of nuclear and cytoplasmic pERK targets, suggesting that the pERK in ORF45 or ORF45/RSK complexes in the nucleus retained their kinase activity.

As a collaborative effort in our laboratory, I performed experiments leading to two figures published in this manuscript and included below. 
Excerpt from our paper, Woodson, et al, 2014:

Previous data from Zhu's laboratory suggest that when KSHV ORF45 is bound to pRSK, the interaction between pRSK and pERK is prolonged leading to the formation of a more stable trimeric complex (170). To assess the stability of the ORF45 nucleated trimeric complexes in even greater detail, we infected RhF for 48 hours, allowing the accumulation of complex-protected pERK, and then determined the half-lives of both pERK2 and pERK1 by measuring their levels following the addition of the MEK inhibitor U0126 or DMSO (Figure A4-1). This drug would block the formation of additional pERK1 and pERK2. We hypothesized that stable and phosphatase-resistant complexes would lead to persistent elevations of pERK2 levels even without ongoing MEK activity. Following the addition of U0126 48h p.i., pERK2 levels remained elevated with a prolonged $t_{1 / 2}$ of approximately 3 hours, likely reflecting a slow off rate of ORF45 from the complex and corroborating our hypothesis (Figure A4-1). Of note, the GAPDH normalized ORF45 levels declined only minimally over the 10 hours and were not statistically different between the DMSO and U0126 treated samples. In marked contrast, the levels of pERK1 decreased rapidly with, a $t_{1 / 2}$ of approximately 25 minutes (Figure A4-1). These latter data suggested that, in infected cells, pERK1, in contrast to pERK2, was freely accessible to the enzymatic activity of MAP kinase phosphatases (MKPs) $(31,32,44)$ 
Figure A4-1. The ORF45-containing complex predominantly protects pERK2 over pERK1. (A) Immunoblots of RRV-infected RhF (48 hour; MOI of 2.5) treated with DMSO (left) or the MEK inhibitor, U0126 (50 $\mu \mathrm{M}$, [right]). 10$15 \%$ of total lysate was loaded per lane. Blots were probed with antibodies to phosphorylated ERK (pERK) and GAPDH. Times indicated above blots represents hours after U0126 treatment. (B) Graphical representation of decay of GAPDH normalized pERK1 and pERK2 levels following the addition of DMSO or U0126. Data are the mean of three independent experiments with error bars reflecting the SEM. $R^{2}$ values indicate the coefficient of determination for each $1^{\text {st }}$ order exponential decay curve. (Of note, the lower MOI of 2.5 helped minimize the degree of lysis between p.i. hours 48 to 58 ). Figure from our own openaccess article. 

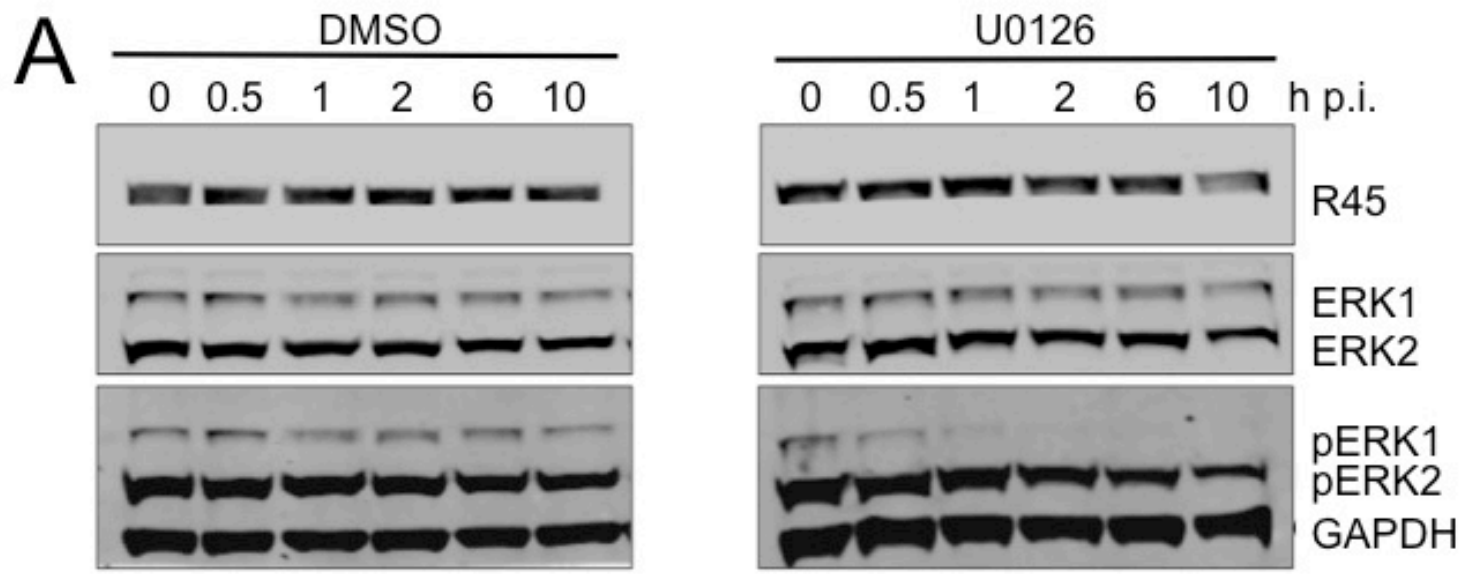

B pERK decay after U0126

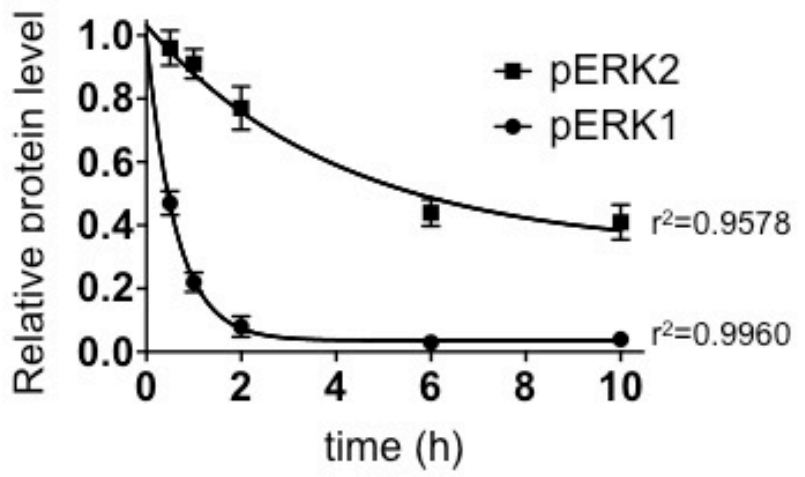


Excerpt from our paper, Woodson, et al, 2014:

If $p R S K$ played a non-essential role in maintenance of $p E R K$ levels during RRV infection, we also predicted that the pERK half-life following the addition of the MEK inhibitor U0126, as we had measured previously (Figure A4-1), would be similar in RRV infected cells following pre-treatment with either control or RSKdirected siRNA. To test this prediction, we infected RhF 24 hours after siRNA reverse-transfection and then waited $48 \mathrm{~h}$ p.i. before adding $\mathrm{U} 0126$ and measuring pERK 1 and 2 over the subsequent 10 hours. We found that the halflives of pERK2 in infected cells pre-treated with siRNA to RSK or siRNA control were statistically indistinguishable and were prolonged $\left(t_{1 / 2}=1.34\right.$ and 1.23 hours, respectively). For pERK1, siRSK pre-treatment actually appeared to lead to a modest increase in half-life compared to siCNL ( $\mathrm{t}_{1 / 2}=40$ vs. 11 minutes, respectively), but both decay curves were statistically distinct from the longer half-lives of pERK2 under either condition (Figure A4-2). Importantly, these data further supported the notion that RSK was not critical to the stability of pERK2 or, therefore, to the ORF45-pERK2 complex. 
Figure A4-2. Delayed decay of pERK2 during RRV infection is independent of RSK expression. (A) RhF were transfected with siCNL or siRSK1+2 (siRSK) and 24 hours later infected with RRV at an $\mathrm{MOI}$ of 2.5. $48 \mathrm{~h}$ p.i. cultures were treated with DMSO (top) or the MEK inhibitor, U0126 (bottom) for up to 10 hours. $10 \mu \mathrm{g}$ of cell lysate $(10-15 \%$ of total lysate) were loaded per lane and immunoblotted with antibodies to pERK, RSK1+2, and GAPDH to control for loading. (B) Graphical representation of two independent experiments described in (A) with levels of pERK1 and pERK2 at each time point first normalized to GAPDH and then expressed as the ratio of their values under U0126 or DMSO conditions. We set the ratios for pERK1 and for pERK2 levels to 1.0 for the siCNL samples at the zero hour time point. Subsequent time points are relative to these initial values. Dashed lines indicate the samples that received SiCNL and solid lines siRSK. Data are the mean from the two experiments with error bars representing the range. $R^{2}$ values indicate the coefficient of determination for each curve and the p-values indicate the level of significance difference using the extra sum-of-squares $\mathrm{F}$ test (Prism 6.0d software) between the decay curves of each pERK1 and pERK2 with (siRSK) or without (siCNL) RSK knockdown ( $p$ values were 0.0029 and 0.799 respectively). Figure from our own open-access article. 


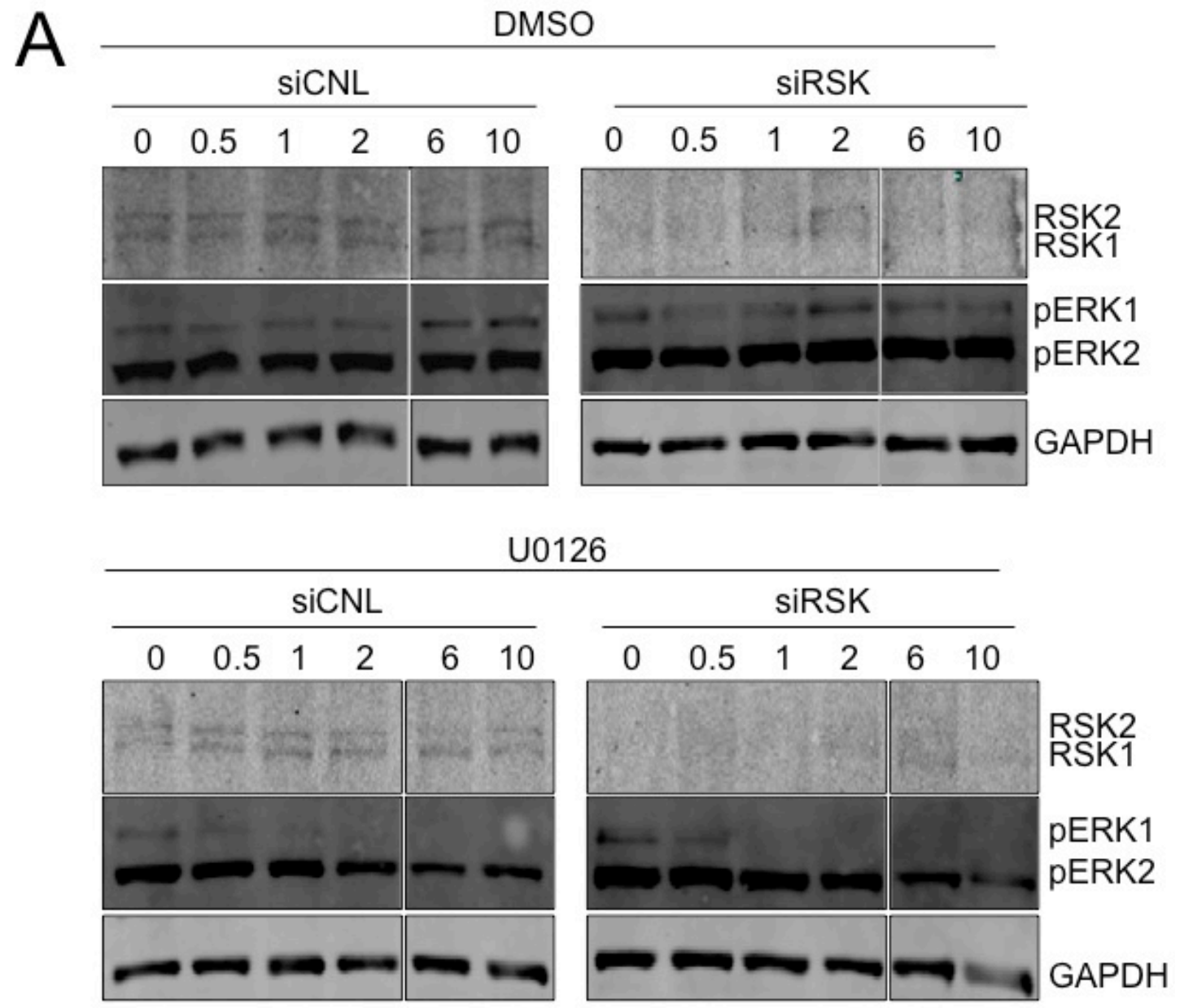

B

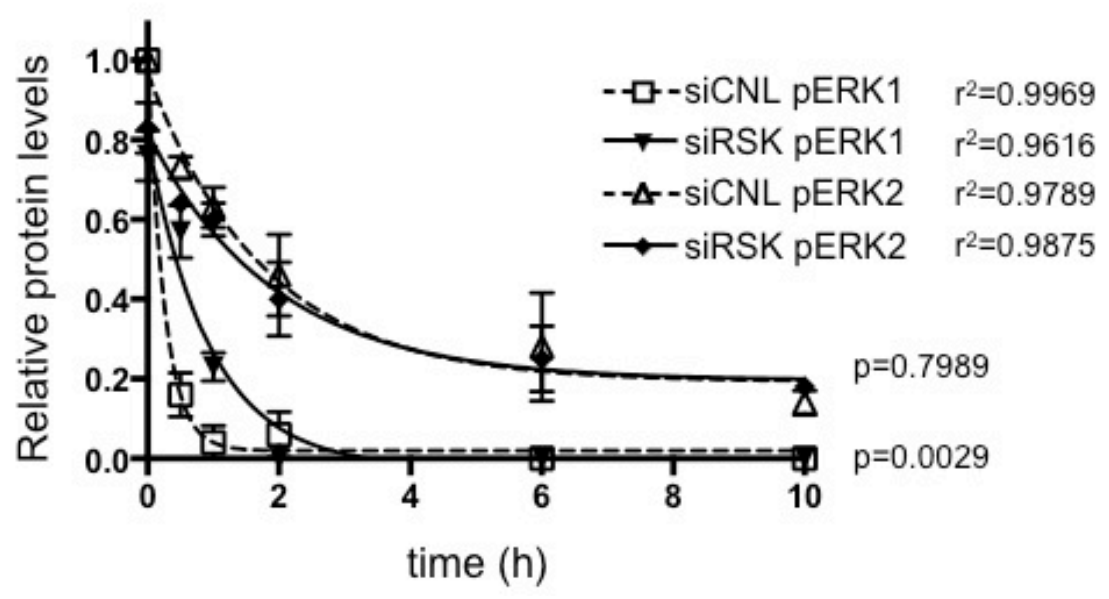


APPENDIX 5. EFFECT OF RRV ORF45 KNOCKDOWN ON VIRUS PRODUCTION 
We have discussed throughout this dissertation, we have discussed that knockdown of ORF52 resulted in particles that were unable to undergo virion morphogenesis in the cytoplasm as well as the release of subviral particles that lacked both ORF52 and ORF45. Additionally, we explored the effect of RRV ORF45 knockdown on ORF52. ORF45 performs several known functions in addition to its structural role in the tegument (see Introduction), including roles in the immediate early phase of viral infection and in DNA replication (361). We predicted that, if knocking down ORF45 affected viral DNA replication, we might see related effects relating to protein expression within the cell.

To investigate the role of ORF45, we transfected RhF cells with a siRNA directed to ORF45 (siORF45) and infected cells 24 hours later with wt RRV at an MOI of $5.48 \mathrm{~h}$ p.i., we collected cell lysates and supernatants. We used a portion of the supernatant for a titer assay and concentrated a portion over a $20 \%$ sucrose cushion to pellet particles.

Upon examining cell lysates for expression of MCP, SCIP, ORF45 and ORF52, we found that our knockdown of ORF45 was very efficient $(>98 \%)$ (Figure A5-1A and 1B) and, additionally, that ORF52 expression decreased to approximately $40 \%$ relative to siCNL. Furthermore, examination of released particles revealed fewer particles released overall in siORF45 conditions, with many lacking ORF52 (Figure A5-1C and 1D). The titer from siORF45-treated RhF cells decreased by over 150 -fold. (Figure A5-1E).

At this point, we can draw only limited conclusions from these experiments regarding the effect of ORF45. ORF52 is a late protein that requires viral DNA 
Figure A5-1. RRV ORF45 plays a role in multiple stages of the RRV lifecycle. (A) Immunoblot analysis of cell lysates from RhF transfected with control siRNA (siCNL), lane 1, or ORF45 siRNA (siORF45), lane 2 and then infected with RRV 24 hours later at MOI 5. Cells were harvested 48 h p.i. and immunoblots were performed, probing for the viral tegument proteins, ORF52 and ORF45, the capsid proteins, MCP and SCIP, and, in addition, ran, to normalize for loading differences. (B) Graphical representation of the intracellular levels of the indicated viral structural proteins after siORF45 treatment relative to siCNL in RRV infected RhF cells. Data represent the mean +/- the range of 2 individual experiments. (C) Supernatants from siCNL or siORF45 treated cells 48 h p.i. collected and concentrated over a $20 \%$ sucrose cushion to isolate particles and equal volumes of media were separated by SDS-PAGE and immunoblotted for MCP, ORF45, SCIP, and ORF52. (D) Graphical representation of the effect of siORF45 relative to siCNL on the levels of the indicated viral protein within the released vira

I particles. Data represent the mean $+/$ - the range of 2 individual experiments. (E) Viral titers in the media $48 \mathrm{~h}$ p.i. from RhF treated with siCNL or siORF45 were determined from 2 different experiments by viral plaque assay. Values are mean +/- the range. 

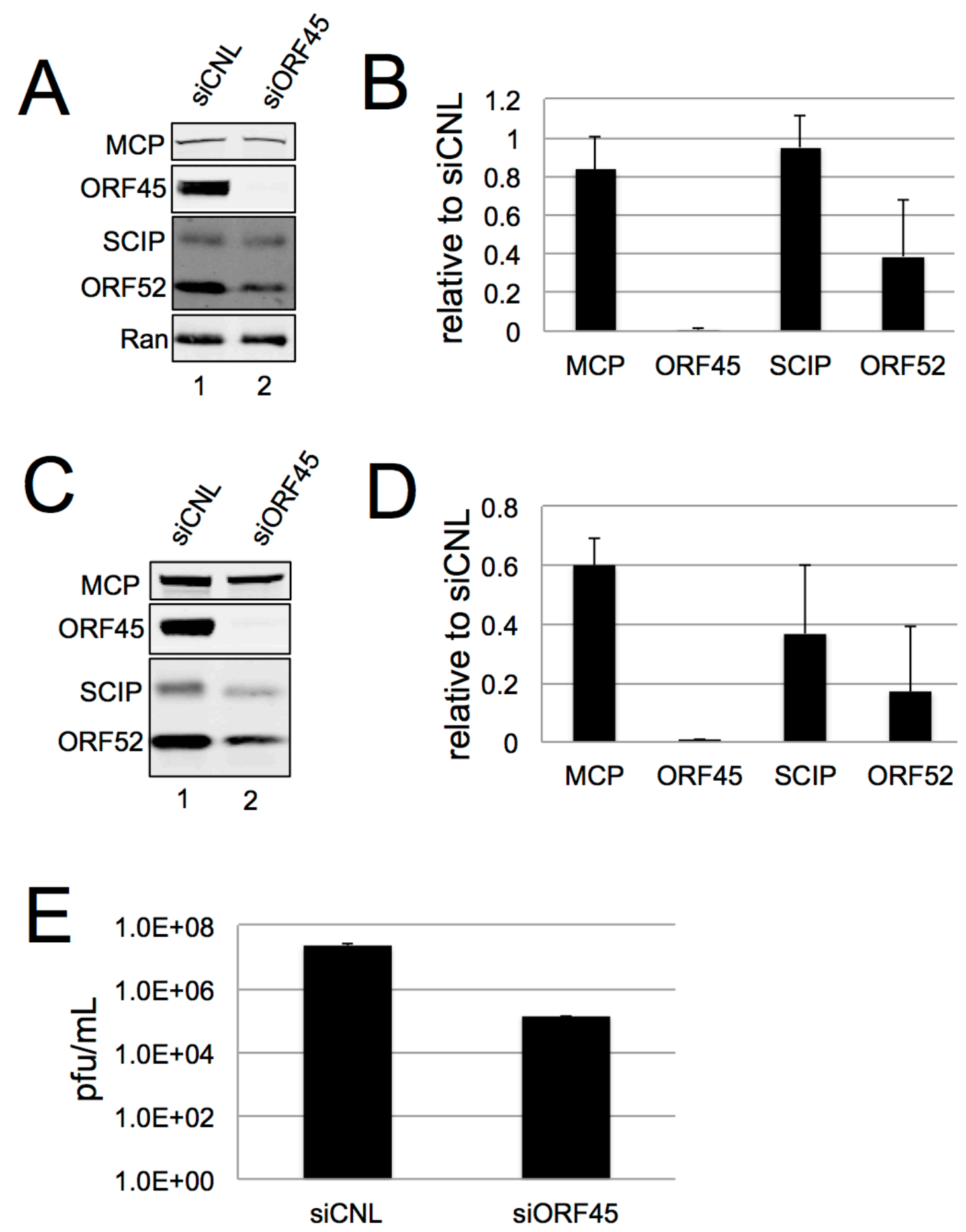
replication prior to its expression, and-based on the western blot of cell lysates-it appears to be affected by an ORF45 knockdown. We did these experiments only in duplicate and do not have qPCR or TEM supporting information. Additionally, as mentioned, ORF45 is a polyfunctional protein that has global effects; and pinpointing its role in virion maturation or early events is not possible without at least corroborating TEM and qPCR. Our laboratory is very interested in the function of RRV ORF45 and will continue to investigate its role in the RRV life cycle. 


\section{REFERENCES}

1. Abada, R., T. Dreyfuss-Grossman, Y. Herman-Bachinsky, H. Geva, S. R. Masa, and R. Sarid. 2008. SIAH-1 interacts with the Kaposi's sarcomaassociated herpesvirus-encoded ORF45 protein and promotes its ubiquitylation and proteasomal degradation. Journal of virology 82:22302240.

2. Adelman, K., B. Salmon, and J. D. Baines. 2001. Herpes simplex virus DNA packaging sequences adopt novel structures that are specifically recognized by a component of the cleavage and packaging machinery. Proceedings of the National Academy of Sciences of the United States of America 98:3086-3091.

3. Adler, H., M. Messerle, and U. H. Koszinowski. 2003. Cloning of herpesviral genomes as bacterial artificial chromosomes. Reviews in medical virology 13:111-121.

4. Adler, H., M. Messerle, M. Wagner, and U. H. Koszinowski. 2000. Cloning and mutagenesis of the murine gammaherpesvirus 68 genome as an infectious bacterial artificial chromosome. Journal of virology 74:6964-6974.

5. Ahn, J. W., K. L. Powell, P. Kellam, and D. G. Alber. 2002. Gammaherpesvirus lytic gene expression as characterized by DNA array. Journal of virology 76:6244-6256.

6. Akula, S. M., N. P. Pramod, F. Z. Wang, and B. Chandran. 2002. Integrin alpha3beta1 (CD 49c/29) is a cellular receptor for Kaposi's sarcomaassociated herpesvirus (KSHV/HHV-8) entry into the target cells. Cell 108:407-419.

7. al-Kobaisi, M. F., F. J. Rixon, I. McDougall, and V. G. Preston. 1991. The herpes simplex virus UL33 gene product is required for the assembly of full capsids. Virology 180:380-388.

8. Alberts, B. 2002. Molecular biology of the cell, 4th ed. Garland Science, New York.

9. Alconada, A., U. Bauer, L. Baudoux, J. Piette, and B. Hoflack. 1998. Intracellular transport of the glycoproteins $\mathrm{gE}$ and $\mathrm{gI}$ of the varicella-zoster virus. gE accelerates the maturation of gI and determines its accumulation in the trans-Golgi network. The Journal of biological chemistry 273:1343013436.

10. Alconada, A., U. Bauer, and B. Hoflack. 1996. A tyrosine-based motif and a casein kinase II phosphorylation site regulate the intracellular trafficking of the varicella-zoster virus glycoprotein I, a protein localized in the trans-Golgi network. The EMBO journal 15:6096-6110.

11. Alconada, A., U. Bauer, B. Sodeik, and B. Hoflack. 1999. Intracellular traffic of herpes simplex virus glycoprotein $\mathrm{gE}$ : characterization of the sorting signals required for its trans-Golgi network localization. Journal of virology 73:377-387.

12. Alexander, L., L. Denekamp, A. Knapp, M. R. Auerbach, B. Damania, and R. C. Desrosiers. 2000. The primary sequence of rhesus monkey rhadinovirus isolate 26-95: sequence similarities to Kaposi's sarcoma- 
associated herpesvirus and rhesus monkey rhadinovirus isolate 17577. Journal of virology 74:3388-3398.

13. Angeletti, P. C., L. Zhang, and C. Wood. 2008. The viral etiology of AIDSassociated malignancies. Adv Pharmacol 56:509-557.

14. Antman, K., and Y. Chang. 2000. Kaposi's sarcoma. The New England journal of medicine 342:1027-1038.

15. Ayscough, K. R., and D. G. Drubin. 1996. ACTIN: general principles from studies in yeast. Annual review of cell and developmental biology 12:129160.

16. Baines, J. D., and B. Roizman. 1991. The open reading frames UL3, UL4, UL10, and UL16 are dispensable for the replication of herpes simplex virus 1 in cell culture. Journal of virology 65:938-944.

17. Baldick, C. J., Jr., and T. Shenk. 1996. Proteins associated with purified human cytomegalovirus particles. Journal of virology 70:6097-6105.

18. Ballestas, M. E., P. A. Chatis, and K. M. Kaye. 1999. Efficient persistence of extrachromosomal KSHV DNA mediated by latency-associated nuclear antigen. Science 284:641-644.

19. Barkalow, K., and J. H. Hartwig. 1995. Actin cytoskeleton. Setting the pace of cell movement. Current biology : CB 5:1000-1002.

20. Baron, S. 1996. Medical microbiology, 4th ed. University of Texas Medical Branch at Galveston, Galveston, Tex.

21. Bayley, A. C. 1984. Aggressive Kaposi's sarcoma in Zambia, 1983. Lancet 1:1318-1320.

22. Bazinet, C., and J. King. 1985. The DNA translocating vertex of dsDNA bacteriophage. Annual review of microbiology 39:109-129.

23. Bechtel, J. T., R. C. Winant, and D. Ganem. 2005. Host and viral proteins in the virion of Kaposi's sarcoma-associated herpesvirus. Journal of virology 79:4952-4964.

24. Benach, J., L. Wang, Y. Chen, C. K. Ho, S. Lee, J. Seetharaman, R. Xiao, T. B. Acton, G. T. Montelione, H. Deng, R. Sun, and L. Tong. 2007. Structural and functional studies of the abundant tegument protein ORF52 from murine gammaherpesvirus 68. The Journal of biological chemistry 282:3153431541.

25. Biernat, J., N. Gustke, G. Drewes, E. M. Mandelkow, and E. Mandelkow. 1993. Phosphorylation of Ser262 strongly reduces binding of tau to microtubules: distinction between PHF-like immunoreactivity and microtubule binding. Neuron 11:153-163.

26. Black, L. W. 1995. DNA packaging and cutting by phage terminases: control in phage $\mathrm{T} 4$ by a synaptic mechanism. BioEssays : news and reviews in molecular, cellular and developmental biology 17:1025-1030.

27. Bortz, E., L. Wang, Q. Jia, T. T. Wu, J. P. Whitelegge, H. Deng, Z. H. Zhou, and R. Sun. 2007. Murine gammaherpesvirus 68 ORF52 encodes a tegument protein required for virion morphogenesis in the cytoplasm. Journal of virology 81:10137-10150. 
28. Bortz, E., J. P. Whitelegge, Q. Jia, Z. H. Zhou, J. P. Stewart, T. T. Wu, and R. Sun. 2003. Identification of proteins associated with murine gammaherpesvirus 68 virions. Journal of virology 77:13425-13432.

29. Boshoff, C., and Y. Chang. 2001. Kaposi's sarcoma-associated herpesvirus: a new DNA tumor virus. Annual review of medicine 52:453-470.

30. Boshoff, C., Y. Endo, P. D. Collins, Y. Takeuchi, J. D. Reeves, V. L. Schweickart, M. A. Siani, T. Sasaki, T. J. Williams, P. W. Gray, P. S. Moore, Y. Chang, and R. A. Weiss. 1997. Angiogenic and HIV-inhibitory functions of KSHV-encoded chemokines. Science 278:290-294.

31. Boutros, T., E. Chevet, and P. Metrakos. 2008. Mitogen-activated protein (MAP) kinase/MAP kinase phosphatase regulation: roles in cell growth, death, and cancer. Pharmacological reviews 60:261-310.

32. Boutros, T., A. Nantel, A. Emadali, G. Tzimas, S. Conzen, E. Chevet, and P. P. Metrakos. 2008. The MAP kinase phosphatase-1 MKP-1/DUSP1 is a regulator of human liver response to transplantation. American journal of transplantation : official journal of the American Society of Transplantation and the American Society of Transplant Surgeons 8:2558-2568.

33. Bowman, B. R., R. L. Welschhans, H. Jayaram, N. D. Stow, V. G. Preston, and F. A. Quiocho. 2006. Structural characterization of the UL25 DNApackaging protein from herpes simplex virus type 1. Journal of virology 80:2309-2317.

34. Brandenburg, B., and X. Zhuang. 2007. Virus trafficking - learning from single-virus tracking. Nature reviews. Microbiology 5:197-208.

35. Brideau, A. D., B. W. Banfield, and L. W. Enquist. 1998. The Us9 gene product of pseudorabies virus, an alphaherpesvirus, is a phosphorylated, tailanchored type II membrane protein. Journal of virology 72:4560-4570.

36. Brown, J. C., and W. W. Newcomb. 2011. Herpesvirus capsid assembly: insights from structural analysis. Current opinion in virology 1:142-149.

37. Cai, Q., S. C. Verma, J. Lu, and E. S. Robertson. 2010. Molecular biology of Kaposi's sarcoma-associated herpesvirus and related oncogenesis. Advances in virus research 78:87-142.

38. Calderwood, M. A., K. Venkatesan, L. Xing, M. R. Chase, A. Vazquez, A. M. Holthaus, A. E. Ewence, N. Li, T. Hirozane-Kishikawa, D. E. Hill, M. Vidal, E. Kieff, and E. Johannsen. 2007. Epstein-Barr virus and virus human protein interaction maps. Proceedings of the National Academy of Sciences of the United States of America 104:7606-7611.

39. Carbone, A., and G. Gaidano. 1997. HHV-8-positive body-cavity-based lymphoma: a novel lymphoma entity. British journal of haematology 97:515522.

40. Cardone, G., W. W. Newcomb, N. Cheng, P. T. Wingfield, B. L. Trus, J. C. Brown, and A. C. Steven. 2012. The UL36 tegument protein of herpes simplex virus 1 has a composite binding site at the capsid vertices. Journal of virology 86:4058-4064. 
41. Casper, C. 2005. The aetiology and management of Castleman disease at 50 years: translating pathophysiology to patient care. British journal of haematology 129:3-17.

42. Catalano, C. E. 2000. The terminase enzyme from bacteriophage lambda: a DNA-packaging machine. Cellular and molecular life sciences : CMLS 57:128148.

43. Catalano, C. E., D. Cue, and M. Feiss. 1995. Virus DNA packaging: the strategy used by phage lambda. Molecular microbiology 16:1075-1086.

44. Caunt, C. J., and S. M. Keyse. 2013. Dual-specificity MAP kinase phosphatases (MKPs): shaping the outcome of MAP kinase signalling. The FEBS journal 280:489-504.

45. Cesarman, E. 2002. The role of Kaposi's sarcoma-associated herpesvirus (KSHV/HHV-8) in lymphoproliferative diseases. Recent results in cancer research. Fortschritte der Krebsforschung. Progres dans les recherches sur le cancer 159:27-37.

46. Cesarman, E., Y. Chang, P. S. Moore, J. W. Said, and D. M. Knowles. 1995. Kaposi's sarcoma-associated herpesvirus-like DNA sequences in AIDSrelated body-cavity-based lymphomas. The New England journal of medicine 332:1186-1191.

47. Cesarman, E., P. S. Moore, P. H. Rao, G. Inghirami, D. M. Knowles, and Y. Chang. 1995. In vitro establishment and characterization of two acquired immunodeficiency syndrome-related lymphoma cell lines (BC-1 and BC-2) containing Kaposi's sarcoma-associated herpesvirus-like (KSHV) DNA sequences. Blood 86:2708-2714.

48. Chakraborty, S., M. V. Veettil, and B. Chandran. 2012. Kaposi's Sarcoma Associated Herpesvirus Entry into Target Cells. Frontiers in microbiology 3:6.

49. Chan, S. R., C. Bloomer, and B. Chandran. 1998. Identification and characterization of human herpesvirus-8 lytic cycle-associated ORF 59 protein and the encoding cDNA by monoclonal antibody. Virology 240:118126.

50. Chandran, B. 2010. Early events in Kaposi's sarcoma-associated herpesvirus infection of target cells. Journal of virology 84:2188-2199.

51. Chang, W. L., and P. A. Barry. 2003. Cloning of the full-length rhesus cytomegalovirus genome as an infectious and self-excisable bacterial artificial chromosome for analysis of viral pathogenesis. Journal of virology 77:5073-5083.

52. Chang, Y., E. Cesarman, M. S. Pessin, F. Lee, J. Culpepper, D. M. Knowles, and P. S. Moore. 1994. Identification of herpesvirus-like DNA sequences in AIDS-associated Kaposi's sarcoma. Science 266:1865-1869.

53. Chang, Y. E., A. P. Poon, and B. Roizman. 1996. Properties of the protein encoded by the UL32 open reading frame of herpes simplex virus 1. Journal of virology 70:3938-3946.

54. Cheeran, M. C., J. R. Lokensgard, and M. R. Schleiss. 2009. Neuropathogenesis of congenital cytomegalovirus infection: disease 
mechanisms and prospects for intervention. Clinical microbiology reviews 22:99-126, Table of Contents.

55. Chen, D. H., H. Jiang, M. Lee, F. Liu, and Z. H. Zhou. 1999. Threedimensional visualization of tegument/capsid interactions in the intact human cytomegalovirus. Virology 260:10-16.

56. Chi, J. H., C. A. Harley, A. Mukhopadhyay, and D. W. Wilson. 2005. The cytoplasmic tail of herpes simplex virus envelope glycoprotein D binds to the tegument protein VP22 and to capsids. The Journal of general virology 86:253-261.

57. Chiu, Y. F., B. Sugden, P. J. Chang, L. W. Chen, Y. J. Lin, Y. C. Lan, C. H. Lai, J. Y. Liou, S. T. Liu, and C. H. Hung. 2012. Characterization and intracellular trafficking of Epstein-Barr virus BBLF1, a protein involved in virion maturation. Journal of virology 86:9647-9655.

58. Cockrell, S. K., M. E. Sanchez, A. Erazo, and F. L. Homa. 2009. Role of the UL25 protein in herpes simplex virus DNA encapsidation. Journal of virology 83:47-57.

59. Coller, K. E., J. I. Lee, A. Ueda, and G. A. Smith. 2007. The capsid and tegument of the alphaherpesviruses are linked by an interaction between the UL25 and VP1/2 proteins. Journal of virology 81:11790-11797.

60. Copeland, A. M., W. W. Newcomb, and J. C. Brown. 2009. Herpes simplex virus replication: roles of viral proteins and nucleoporins in capsid-nucleus attachment. Journal of virology 83:1660-1668.

61. Crump, C. M., B. Bruun, S. Bell, L. E. Pomeranz, T. Minson, and H. M. Browne. 2004. Alphaherpesvirus glycoprotein M causes the relocalization of plasma membrane proteins. The Journal of general virology 85:3517-3527.

62. Dai, W., Q. Jia, E. Bortz, S. Shah, J. Liu, I. Atanasov, X. Li, K. A. Taylor, R. Sun, and Z. H. Zhou. 2008. Unique structures in a tumor herpesvirus revealed by cryo-electron tomography and microscopy. Journal of structural biology 161:428-438.

63. Damania, B., J. K. Choi, and J. U. Jung. 2000. Signaling activities of gammaherpesvirus membrane proteins. Journal of virology 74:1593-1601.

64. Das, S., and P. E. Pellett. 2011. Spatial relationships between markers for secretory and endosomal machinery in human cytomegalovirus-infected cells versus those in uninfected cells. Journal of virology 85:5864-5879.

65. Das, S., A. Vasanji, and P. E. Pellett. 2007. Three-dimensional structure of the human cytomegalovirus cytoplasmic virion assembly complex includes a reoriented secretory apparatus. Journal of virology 81:11861-11869.

66. Davison, M. D., F. J. Rixon, and A. J. Davison. 1992. Identification of genes encoding two capsid proteins (VP24 and VP26) of herpes simplex virus type 1. The Journal of general virology 73 ( Pt 10):2709-2713.

67. Delorme-Axford, E., and C. B. Coyne. 2011. The actin cytoskeleton as a barrier to virus infection of polarized epithelial cells. Viruses 3:2462-2477.

68. Deng, B., C. M. O'Connor, D. H. Kedes, and Z. H. Zhou. 2007. Direct visualization of the putative portal in the Kaposi's sarcoma-associated 
herpesvirus capsid by cryoelectron tomography. Journal of virology 81:3640-3644.

69. Desrosiers, R. C., V. G. Sasseville, S. C. Czajak, X. Zhang, K. G. Mansfield, A. Kaur, R. P. Johnson, A. A. Lackner, and J. U. Jung. 1997. A herpesvirus of rhesus monkeys related to the human Kaposi's sarcoma-associated herpesvirus. Journal of virology 71:9764-9769.

70. DeWire, S. M., M. A. McVoy, and B. Damania. 2002. Kinetics of expression of rhesus monkey rhadinovirus (RRV) and identification and characterization of a polycistronic transcript encoding the RRV Orf50/Rta, RRV R8, and R8.1 genes. Journal of virology 76:9819-9831.

71. DeWire, S. M., E. S. Money, S. P. Krall, and B. Damania. 2003. Rhesus monkey rhadinovirus (RRV): construction of a RRV-GFP recombinant virus and development of assays to assess viral replication. Virology 312:122-134.

72. DiGiovanna, J. J., and B. Safai. 1981. Kaposi's sarcoma. Retrospective study of 90 cases with particular emphasis on the familial occurrence, ethnic background and prevalence of other diseases. The American journal of medicine 71:779-783.

73. Dittmer, D. P., C. M. Gonzalez, W. Vahrson, S. M. DeWire, R. Hines-Boykin, and B. Damania. 2005. Whole-genome transcription profiling of rhesus monkey rhadinovirus. Journal of virology 79:8637-8650.

74. Dodding, M. P., and M. Way. 2011. Coupling viruses to dynein and kinesin-1. The EMBO journal 30:3527-3539.

75. Dohner, K., and B. Sodeik. 2005. The role of the cytoskeleton during viral infection. Current topics in microbiology and immunology 285:67-108.

76. Dohner, K., A. Wolfstein, U. Prank, C. Echeverri, D. Dujardin, R. Vallee, and B. Sodeik. 2002. Function of dynein and dynactin in herpes simplex virus capsid transport. Molecular biology of the cell 13:2795-2809.

77. Douglas, J. L., J. K. Gustin, K. Viswanathan, M. Mansouri, A. V. Moses, and K. Fruh. 2010. The great escape: viral strategies to counter BST-2/tetherin. PLoS pathogens 6:e1000913.

78. Douglas, J. L., K. Viswanathan, M. N. McCarroll, J. K. Gustin, K. Fruh, and A. V. Moses. 2009. Vpu directs the degradation of the human immunodeficiency virus restriction factor BST-2/Tetherin via a \{beta\}TrCPdependent mechanism. Journal of virology 83:7931-7947.

79. Downing, R. G., R. P. Eglin, and A. C. Bayley. 1984. African Kaposi's sarcoma and AIDS. Lancet 1:478-480.

80. Drechsel, D. N., A. A. Hyman, M. H. Cobb, and M. W. Kirschner. 1992. Modulation of the dynamic instability of tubulin assembly by the microtubule-associated protein tau. Molecular biology of the cell 3:11411154.

81. Drewes, G., A. Ebneth, and E. M. Mandelkow. 1998. MAPs, MARKs and microtubule dynamics. Trends in biochemical sciences 23:307-311.

82. Duarte, M., L. Wang, M. A. Calderwood, G. Adelmant, M. Ohashi, J. Roecklein-Canfield, J. A. Marto, D. E. Hill, H. Deng, and E. Johannsen. 2013. An RS motif within the Epstein-Barr virus BLRF2 tegument protein is 
phosphorylated by SRPK2 and is important for viral replication. PloS one 8:e53512.

83. Durack, D. T. 1981. Opportunistic infections and Kaposi's sarcoma in homosexual men. The New England journal of medicine 305:1465-1467.

84. Ebrahimi, B., B. M. Dutia, K. L. Roberts, J. J. Garcia-Ramirez, P. Dickinson, J. P. Stewart, P. Ghazal, D. J. Roy, and A. A. Nash. 2003. Transcriptome profile of murine gammaherpesvirus-68 lytic infection. The Journal of general virology 84:99-109.

85. El-Osta, H. E., and R. Kurzrock. 2011. Castleman's disease: from basic mechanisms to molecular therapeutics. The oncologist 16:497-511.

86. Elliott, G., and P. O'Hare. 1998. Herpes simplex virus type 1 tegument protein VP22 induces the stabilization and hyperacetylation of microtubules. Journal of virology 72:6448-6455.

87. Estep, R. D., M. F. Powers, B. K. Yen, H. Li, and S. W. Wong. 2007. Construction of an infectious rhesus rhadinovirus bacterial artificial chromosome for the analysis of Kaposi's sarcoma-associated herpesvirusrelated disease development. Journal of virology 81:2957-2969.

88. Evans, D. T., R. Serra-Moreno, R. K. Singh, and J. C. Guatelli. 2010. BST2/tetherin: a new component of the innate immune response to enveloped viruses. Trends in microbiology 18:388-396.

89. Farge, D. 1993. Kaposi's sarcoma in organ transplant recipients. The Collaborative Transplantation Research Group of Ile de France. The European journal of medicine 2:339-343.

90. Feierbach, B., S. Piccinotti, M. Bisher, W. Denk, and L. W. Enquist. 2006. Alpha-herpesvirus infection induces the formation of nuclear actin filaments. PLoS pathogens 2:e85.

91. Fewell, G. D., and K. Schmitt. 2006. Vector-based RNAi approaches for stable, inducible and genome-wide screens. Drug discovery today 11:975982.

92. Fields, B. N., P. M. Howley, and D. M. Knipe. 2007. Fields' virology, 5th ed. Wolters Kluwer Health/Lippincott Williams \& Wilkins, Philadelphia.

93. Flore, O., S. Rafii, S. Ely, J. J. O'Leary, E. M. Hyjek, and E. Cesarman. 1998. Transformation of primary human endothelial cells by Kaposi's sarcomaassociated herpesvirus. Nature 394:588-592.

94. Foreman, K. E., J. Friborg, Jr., W. P. Kong, C. Woffendin, P. J. Polverini, B. J. Nickoloff, and G. J. Nabel. 1997. Propagation of a human herpesvirus from AIDS-associated Kaposi's sarcoma. The New England journal of medicine 336:163-171.

95. Foster, T. P., J. M. Melancon, T. L. Olivier, and K. G. Kousoulas. 2004. Herpes simplex virus type 1 glycoprotein $\mathrm{K}$ and the UL20 protein are interdependent for intracellular trafficking and trans-Golgi network localization. Journal of virology 78:13262-13277.

96. Friedman-Kien, A. E., Laubenstein, L., Marmor, M., et al. 1981. Kaposi's sarcoma and Pneumocystis pneumonia among homosexual men--New York 
City and California. MMWR. Morbidity and mortality weekly report 30:305-308.

97. Friedman-Kien, A. E., and B. R. Saltzman. 1990. Clinical manifestations of classical, endemic African, and epidemic AIDS-associated Kaposi's sarcoma. Journal of the American Academy of Dermatology 22:1237-1250.

98. Fu, X. D. 1995. The superfamily of arginine/serine-rich splicing factors. RNA 1:663-680.

99. Fuchs, E. 1994. Intermediate filaments and disease: mutations that cripple cell strength. The Journal of cell biology 125:511-516.

100. Fuchs, W., B. G. Klupp, H. Granzow, C. Hengartner, A. Brack, A. Mundt, L. W. Enquist, and T. C. Mettenleiter. 2002. Physical interaction between envelope glycoproteins $\mathrm{E}$ and $\mathrm{M}$ of pseudorabies virus and the major tegument protein UL49. Journal of virology 76:8208-8217.

101. Fuchs, W., B. G. Klupp, H. Granzow, N. Osterrieder, and T. C. Mettenleiter. 2002. The interacting UL31 and UL34 gene products of pseudorabies virus are involved in egress from the host-cell nucleus and represent components of primary enveloped but not mature virions. Journal of virology 76:364-378.

102. Full, F., D. Jungnickl, N. Reuter, E. Bogner, K. Brulois, B. Scholz, M. Sturzl, J. Myoung, J. U. Jung, T. Stamminger, and A. Ensser. 2014. Kaposi's sarcoma associated herpesvirus tegument protein ORF75 is essential for viral lytic replication and plays a critical role in the antagonization of ND10instituted intrinsic immunity. PLoS pathogens 10:e1003863.

103. Gallo, M. L., D. I. Dorsky, C. S. Crumpacker, and D. S. Parris. 1989. The essential 65-kilodalton DNA-binding protein of herpes simplex virus stimulates the virus-encoded DNA polymerase. Journal of virology 63:50235029.

104. Gange, R. W., and E. W. Jones. 1978. Kaposi's sarcoma and immunosuppressive therapy: an appraisal. Clinical and experimental dermatology 3:135-146.

105. Gao, M., L. Matusick-Kumar, W. Hurlburt, S. F. DiTusa, W. W. Newcomb, J. C. Brown, P. J. McCann, 3rd, I. Deckman, and R. J. Colonno. 1994. The protease of herpes simplex virus type 1 is essential for functional capsid formation and viral growth. Journal of virology 68:3702-3712.

106. Garber, D. A., S. M. Beverley, and D. M. Coen. 1993. Demonstration of circularization of herpes simplex virus DNA following infection using pulsed field gel electrophoresis. Virology 197:459-462.

107. Garrigues, H. J., Y. E. Rubinchikova, C. M. Dipersio, and T. M. Rose. 2008. Integrin alphaVbeta3 Binds to the RGD motif of glycoprotein B of Kaposi's sarcoma-associated herpesvirus and functions as an RGD-dependent entry receptor. Journal of virology 82:1570-1580.

108. Gaspar, M., M. B. Gill, J. B. Losing, J. S. May, and P. G. Stevenson. 2008. Multiple functions for ORF75c in murid herpesvirus-4 infection. PloS one 3:e2781. 
109. Giannakouros, T., E. Nikolakaki, I. Mylonis, and E. Georgatsou. 2011.

Serine-arginine protein kinases: a small protein kinase family with a large cellular presence. The FEBS journal 278:570-586.

110. Gibson, W., and B. Roizman. 1972. Proteins specified by herpes simplex virus. 8. Characterization and composition of multiple capsid forms of subtypes 1 and 2. Journal of virology 10:1044-1052.

111. Goodson, H. V., C. Valetti, and T. E. Kreis. 1997. Motors and membrane traffic. Current opinion in cell biology 9:18-28.

112. Granzow, H., B. G. Klupp, W. Fuchs, J. Veits, N. Osterrieder, and T. C. Mettenleiter. 2001. Egress of alphaherpesviruses: comparative ultrastructural study. Journal of virology 75:3675-3684.

113. Granzow, H., B. G. Klupp, and T. C. Mettenleiter. 2005. Entry of pseudorabies virus: an immunogold-labeling study. Journal of virology 79:3200-3205.

114. Granzow, H., F. Weiland, A. Jons, B. G. Klupp, A. Karger, and T. C. Mettenleiter. 1997. Ultrastructural analysis of the replication cycle of pseudorabies virus in cell culture: a reassessment. Journal of virology 71:2072-2082.

115. Grayson, W., and L. Pantanowitz. 2008. Histological variants of cutaneous Kaposi sarcoma. Diagnostic pathology 3:31.

116. Greber, U. F., and M. Way. 2006. A superhighway to virus infection. Cell 124:741-754.

117. Grinde, B. 2013. Herpesviruses: latency and reactivation - viral strategies and host response. Journal of oral microbiology 5.

118. Grose, C. 1990. Glycoproteins encoded by varicella-zoster virus: biosynthesis, phosphorylation, and intracellular trafficking. Annual review of microbiology 44:59-80.

119. Guo, H., S. Shen, L. Wang, and H. Deng. 2010. Role of tegument proteins in herpesvirus assembly and egress. Protein \& cell 1:987-998.

120. Guo, H., L. Wang, L. Peng, Z. H. Zhou, and H. Deng. 2009. Open reading frame 33 of a gammaherpesvirus encodes a tegument protein essential for virion morphogenesis and egress. Journal of virology 83:10582-10595.

121. Haarr, L., and S. Skulstad. 1994. The herpes simplex virus type 1 particle: structure and molecular functions. Review article. APMIS : acta pathologica, microbiologica, et immunologica Scandinavica 102:321-346.

122. Hahn, A. S., J. K. Kaufmann, E. Wies, E. Naschberger, J. Panteleev-Ivlev, K. Schmidt, A. Holzer, M. Schmidt, J. Chen, S. Konig, A. Ensser, J. Myoung, N. H. Brockmeyer, M. Sturzl, B. Fleckenstein, and F. Neipel. 2012. The ephrin receptor tyrosine kinase A2 is a cellular receptor for Kaposi's sarcomaassociated herpesvirus. Nature medicine 18:961-966.

123. Hassman, L. M., T. J. Ellison, and D. H. Kedes. 2011. KSHV infects a subset of human tonsillar B cells, driving proliferation and plasmablast differentiation. The Journal of clinical investigation 121:752-768.

124. He, B., M. Gross, and B. Roizman. 1997. The gamma(1)34.5 protein of herpes simplex virus 1 complexes with protein phosphatase 1alpha to 
dephosphorylate the alpha subunit of the eukaryotic translation initiation factor 2 and preclude the shutoff of protein synthesis by doublestranded RNA-activated protein kinase. Proceedings of the National Academy of Sciences of the United States of America 94:843-848.

125. Heldwein, E. E., and C. Krummenacher. 2008. Entry of herpesviruses into mammalian cells. Cellular and molecular life sciences : CMLS 65:1653-1668.

126. Henaff, D., K. Radtke, and R. Lippe. 2012. Herpesviruses exploit several host compartments for envelopment. Traffic 13:1443-1449.

127. Hengge, U. R., T. Ruzicka, S. K. Tyring, M. Stuschke, M. Roggendorf, R. A. Schwartz, and S. Seeber. 2002. Update on Kaposi's sarcoma and other HHV8 associated diseases. Part 1: epidemiology, environmental predispositions, clinical manifestations, and therapy. The Lancet infectious diseases 2:281292.

128. Hengge, U. R., T. Ruzicka, S. K. Tyring, M. Stuschke, M. Roggendorf, R. A. Schwartz, and S. Seeber. 2002. Update on Kaposi's sarcoma and other HHV8 associated diseases. Part 2: pathogenesis, Castleman's disease, and pleural effusion lymphoma. The Lancet infectious diseases 2:344-352.

129. Hirokawa, N. 2000. Stirring up development with the heterotrimeric kinesin KIF3. Traffic 1:29-34.

130. Hirokawa, N., Y. Noda, Y. Tanaka, and S. Niwa. 2009. Kinesin superfamily motor proteins and intracellular transport. Nature reviews. Molecular cell biology 10:682-696.

131. Hollinshead, M., H. L. Johns, C. L. Sayers, C. Gonzalez-Lopez, G. L. Smith, and G. Elliott. 2012. Endocytic tubules regulated by Rab GTPases 5 and 11 are used for envelopment of herpes simplex virus. The EMBO journal 31:4204-4220.

132. Holzenburg, A., E. Bogner, and Ebrary Academic Complete Subscription Collection. 2002. Structure-function relationships of human pathogenic viruses. Kluwer Academic/Plenum Publishers, New York.

133. Homa, F. L., and J. C. Brown. 1997. Capsid assembly and DNA packaging in herpes simplex virus. Reviews in medical virology 7:107-122.

134. Hong, Y., C. B. Chan, I. S. Kwon, X. Li, M. Song, H. P. Lee, X. Liu, P. Sompol, P. Jin, H. G. Lee, S. P. Yu, and K. Ye. 2012. SRPK2 phosphorylates tau and mediates the cognitive defects in Alzheimer's disease. The Journal of neuroscience : the official journal of the Society for Neuroscience 32:1726217272.

135. Humphries, J. D., A. Byron, and M. J. Humphries. 2006. Integrin ligands at a glance. Journal of cell science 119:3901-3903.

136. Illenberger, S., Q. Zheng-Fischhofer, U. Preuss, K. Stamer, K. Baumann, B. Trinczek, J. Biernat, R. Godemann, E. M. Mandelkow, and E. Mandelkow. 1998. The endogenous and cell cycle-dependent phosphorylation of tau protein in living cells: implications for Alzheimer's disease. Molecular biology of the cell 9:1495-1512.

137. Iscovich, J., P. Boffetta, S. Franceschi, E. Azizi, and R. Sarid. 2000. Classic kaposi sarcoma: epidemiology and risk factors. Cancer 88:500-517. 
138. Jang, S. W., X. Liu, H. Fu, H. Rees, M. Yepes, A. Levey, and K. Ye. 2009. Interaction of Akt-phosphorylated SRPK2 with 14-3-3 mediates cell cycle and cell death in neurons. The Journal of biological chemistry 284:24512-24525.

139. Jang, S. W., S. J. Yang, A. Ehlen, S. Dong, H. Khoury, J. Chen, J. L. Persson, and K. Ye. 2008. Serine/arginine protein-specific kinase 2 promotes leukemia cell proliferation by phosphorylating acinus and regulating cyclin A1. Cancer research 68:4559-4570.

140. Jarvis, M. A., K. N. Fish, C. Soderberg-Naucler, D. N. Streblow, H. L. Meyers, G. Thomas, and J. A. Nelson. 2002. Retrieval of human cytomegalovirus glycoprotein B from cell surface is not required for virus envelopment in astrocytoma cells. Journal of virology 76:5147-5155.

141. Jia, Q., V. Chernishof, E. Bortz, I. McHardy, T. T. Wu, H. I. Liao, and R. Sun. 2005. Murine gammaherpesvirus 68 open reading frame 45 plays an essential role during the immediate-early phase of viral replication. Journal of virology 79:5129-5141.

142. Johannsen, E., M. Luftig, M. R. Chase, S. Weicksel, E. Cahir-McFarland, D. Illanes, D. Sarracino, and E. Kieff. 2004. Proteins of purified Epstein-Barr virus. Proceedings of the National Academy of Sciences of the United States of America 101:16286-16291.

143. Johnson, D. C., and J. D. Baines. 2011. Herpesviruses remodel host membranes for virus egress. Nature reviews. Microbiology 9:382-394.

144. Jones, P. C., and B. Roizman. 1979. Regulation of herpesvirus macromolecular synthesis. VIII. The transcription program consists of three phases during which both extent of transcription and accumulation of RNA in the cytoplasm are regulated. Journal of virology 31:299-314.

145. Jones, T. R., and S. W. Lee. 2004. An acidic cluster of human cytomegalovirus UL99 tegument protein is required for trafficking and function. Journal of virology 78:1488-1502.

146. Jouvenet, N., S. J. Neil, M. Zhadina, T. Zang, Z. Kratovac, Y. Lee, M. McNatt, T. Hatziioannou, and P. D. Bieniasz. 2009. Broad-spectrum inhibition of retroviral and filoviral particle release by tetherin. Journal of virology 83:1837-1844.

147. Jurak, I., L. B. Silverstein, M. Sharma, and D. M. Coen. 2012. Herpes simplex virus is equipped with RNA- and protein-based mechanisms to repress expression of ATRX, an effector of intrinsic immunity. Journal of virology 86:10093-10102.

148. Kaleeba, J. A., and E. A. Berger. 2006. Kaposi's sarcoma-associated herpesvirus fusion-entry receptor: cystine transporter xCT. Science 311:1921-1924.

149. Kalejta, R. F. 2008. Tegument proteins of human cytomegalovirus. Microbiology and molecular biology reviews : MMBR 72:249-265, table of contents.

150. Kaletsky, R. L., J. R. Francica, C. Agrawal-Gamse, and P. Bates. 2009. Tetherin-mediated restriction of filovirus budding is antagonized by the 
Ebola glycoprotein. Proceedings of the National Academy of Sciences of the United States of America 106:2886-2891.

151. Kamen, D. E., S. T. Gross, M. E. Girvin, and D. W. Wilson. 2005. Structural basis for the physiological temperature dependence of the association of VP16 with the cytoplasmic tail of herpes simplex virus glycoprotein $\mathrm{H}$. Journal of virology 79:6134-6141.

152. Kanai, Y., R. Takemura, T. Oshima, H. Mori, Y. Ihara, M. Yanagisawa, T. Masaki, and N. Hirokawa. 1989. Expression of multiple tau isoforms and microtubule bundle formation in fibroblasts transfected with a single tau cDNA. The Journal of cell biology 109:1173-1184.

153. Kanda, T., M. Yajima, N. Ahsan, M. Tanaka, and K. Takada. 2004. Production of high-titer Epstein-Barr virus recombinants derived from Akata cells by using a bacterial artificial chromosome system. Journal of virology 78:7004-7015.

154. Kaposi, M. K. 1872. Idiopathisches multiples Pigementsarkom der Haut. Arch Dermatol Syph 265.

155. Karczewski, M. K., and K. Strebel. 1996. Cytoskeleton association and virion incorporation of the human immunodeficiency virus type 1 Vif protein. Journal of virology 70:494-507.

156. Kato, A., M. Yamamoto, T. Ohno, M. Tanaka, T. Sata, Y. Nishiyama, and Y. Kawaguchi. 2006. Herpes simplex virus 1-encoded protein kinase UL13 phosphorylates viral Us3 protein kinase and regulates nuclear localization of viral envelopment factors UL34 and UL31. Journal of virology 80:1476-1486.

157. Kato, K., T. Daikoku, F. Goshima, H. Kume, K. Yamaki, and Y. Nishiyama. 2000. Synthesis, subcellular localization and VP16 interaction of the herpes simplex virus type 2 UL46 gene product. Archives of virology 145:21492162.

158. Kattenhorn, L. M., R. Mills, M. Wagner, A. Lomsadze, V. Makeev, M. Borodovsky, H. L. Ploegh, and B. M. Kessler. 2004. Identification of proteins associated with murine cytomegalovirus virions. Journal of virology 78:11187-11197.

159. Keil, G., B. Fleckenstein, and W. Bodemer. 1983. Structural proteins of Herpesvirus saimiri. Journal of virology 47:463-470.

160. Kelly, B. J., C. Fraefel, A. L. Cunningham, and R. J. Diefenbach. 2009. Functional roles of the tegument proteins of herpes simplex virus type 1 . Virus research 145:173-186.

161. Kerur, N., M. V. Veettil, N. Sharma-Walia, S. Sadagopan, V. Bottero, A. G. Paul, and B. Chandran. 2010. Characterization of entry and infection of monocytic THP-1 cells by Kaposi's sarcoma associated herpesvirus (KSHV): role of heparan sulfate, DC-SIGN, integrins and signaling. Virology 406:103116.

162. Klupp, B. G., S. Bottcher, H. Granzow, M. Kopp, and T. C. Mettenleiter. 2005. Complex formation between the UL16 and UL21 tegument proteins of pseudorabies virus. Journal of virology 79:1510-1522. 
163. Klupp, B. G., H. Granzow, and T. C. Mettenleiter. 2000. Primary envelopment of pseudorabies virus at the nuclear membrane requires the UL34 gene product. Journal of virology 74:10063-10073.

164. Kopp, M., H. Granzow, W. Fuchs, B. G. Klupp, E. Mundt, A. Karger, and T. C. Mettenleiter. 2003. The pseudorabies virus UL11 protein is a virion component involved in secondary envelopment in the cytoplasm. Journal of virology 77:5339-5351.

165. Koslowski, K. M., P. R. Shaver, J. T. Casey, 2nd, T. Wilson, G. Yamanaka, A. K. Sheaffer, D. J. Tenney, and N. E. Pederson. 1999. Physical and functional interactions between the herpes simplex virus UL15 and UL28 DNA cleavage and packaging proteins. Journal of virology 73:1704-1707.

166. Krishnan, H. H., N. Sharma-Walia, L. Zeng, S. J. Gao, and B. Chandran. 2005. Envelope glycoprotein gB of Kaposi's sarcoma-associated herpesvirus is essential for egress from infected cells. Journal of virology 79:1095210967.

167. Krummenacher, C., A. Carfi, R. J. Eisenberg, and G. H. Cohen. 2013. Entry of herpesviruses into cells: the enigma variations. Advances in experimental medicine and biology 790:178-195.

168. Kuang, E., B. Fu, Q. Liang, J. Myoung, and F. Zhu. 2011. Phosphorylation of eukaryotic translation initiation factor 4B (EIF4B) by open reading frame 45/p90 ribosomal S6 kinase (ORF45/RSK) signaling axis facilitates protein translation during Kaposi sarcoma-associated herpesvirus (KSHV) lytic replication. The Journal of biological chemistry 286:41171-41182.

169. Kuang, E., Q. Tang, G. G. Maul, and F. Zhu. 2008. Activation of p90 ribosomal S6 kinase by ORF45 of Kaposi's sarcoma-associated herpesvirus and its role in viral lytic replication. Journal of virology 82:1838-1850.

170. Kuang, E., F. Wu, and F. Zhu. 2009. Mechanism of sustained activation of ribosomal S6 kinase (RSK) and ERK by kaposi sarcoma-associated herpesvirus ORF45: multiprotein complexes retain active phosphorylated ERK AND RSK and protect them from dephosphorylation. The Journal of biological chemistry 284:13958-13968.

171. Kuppers, R. 2003. B cells under influence: transformation of B cells by Epstein-Barr virus. Nature reviews. Immunology 3:801-812.

172. Kupzig, S., V. Korolchuk, R. Rollason, A. Sugden, A. Wilde, and G. Banting. 2003. Bst-2/HM1.24 is a raft-associated apical membrane protein with an unusual topology. Traffic 4:694-709.

173. Lamberti, C., and S. K. Weller. 1998. The herpes simplex virus type 1 cleavage/packaging protein, UL32, is involved in efficient localization of capsids to replication compartments. Journal of virology 72:2463-2473.

174. Lee, E. C., D. Yu, J. Martinez de Velasco, L. Tessarollo, D. A. Swing, D. L. Court, N. A. Jenkins, and N. G. Copeland. 2001. A highly efficient Escherichia coli-based chromosome engineering system adapted for recombinogenic targeting and subcloning of BAC DNA. Genomics 73:56-65.

175. Lee, G., and R. Brandt. 1992. Microtubule-bundling studies revisited: is there a role for MAPs? Trends in cell biology 2:286-289. 
176. Leuzinger, H., U. Ziegler, E. M. Schraner, C. Fraefel, D. L. Glauser, I. Heid, M. Ackermann, M. Mueller, and P. Wild. 2005. Herpes simplex virus 1 envelopment follows two diverse pathways. Journal of virology 79:1304713059.

177. Li, X., and F. Zhu. 2009. Identification of the nuclear export and adjacent nuclear localization signals for ORF45 of Kaposi's sarcoma-associated herpesvirus. Journal of virology 83:2531-2539.

178. Liang, Q., B. Fu, F. Wu, X. Li, Y. Yuan, and F. Zhu. 2012. ORF45 of Kaposi's sarcoma-associated herpesvirus inhibits phosphorylation of interferon regulatory factor 7 by IKKepsilon and TBK1 as an alternative substrate. Journal of virology 86:10162-10172.

179. Limaye, A. P., K. A. Kirby, G. D. Rubenfeld, W. M. Leisenring, E. M. Bulger, M. J. Neff, N. S. Gibran, M. L. Huang, T. K. Santo Hayes, L. Corey, and M. Boeckh. 2008. Cytomegalovirus reactivation in critically ill immunocompetent patients. JAMA : the journal of the American Medical Association 300:413-422.

180. Ling, P. D., J. Tan, J. Sewatanon, and R. Peng. 2008. Murine gammaherpesvirus 68 open reading frame 75c tegument protein induces the degradation of PML and is essential for production of infectious virus. Journal of virology 82:8000-8012.

181. Liu, F. Y., and B. Roizman. 1991. The herpes simplex virus 1 gene encoding a protease also contains within its coding domain the gene encoding the more abundant substrate. Journal of virology 65:5149-5156.

182. Loomis, J. S., J. B. Bowzard, R. J. Courtney, and J. W. Wills. 2001. Intracellular trafficking of the UL11 tegument protein of herpes simplex virus type 1. Journal of virology 75:12209-12219.

183. Loomis, J. S., R. J. Courtney, and J. W. Wills. 2003. Binding partners for the UL11 tegument protein of herpes simplex virus type 1. Journal of virology 77:11417-11424.

184. Loret, S., G. Guay, and R. Lippe. 2008. Comprehensive characterization of extracellular herpes simplex virus type 1 virions. Journal of virology 82:8605-8618.

185. Lu, M., J. Suen, C. Frias, R. Pfeiffer, M. H. Tsai, E. Chuang, and S. L. Zeichner. 2004. Dissection of the Kaposi's sarcoma-associated herpesvirus gene expression program by using the viral DNA replication inhibitor cidofovir. Journal of virology 78:13637-13652.

186. Luduena, R. F., and M. C. Roach. 1991. Tubulin sulfhydryl groups as probes and targets for antimitotic and antimicrotubule agents. Pharmacology \& therapeutics 49:133-152.

187. Luxton, G. W., S. Haverlock, K. E. Coller, S. E. Antinone, A. Pincetic, and G. A. Smith. 2005. Targeting of herpesvirus capsid transport in axons is coupled to association with specific sets of tegument proteins. Proceedings of the National Academy of Sciences of the United States of America 102:58325837. 
188. Lyman, M. G., and L. W. Enquist. 2009. Herpesvirus interactions with the host cytoskeleton. Journal of virology 83:2058-2066.

189. Maccioni, R. B., and V. Cambiazo. 1995. Role of microtubule-associated proteins in the control of microtubule assembly. Physiological reviews 75:835-864.

190. MacRae, T. H. 1992. Towards an understanding of microtubule function and cell organization: an overview. Biochemistry and cell biology $=$ Biochimie et biologie cellulaire 70:835-841.

191. Manjunath, N., H. Wu, S. Subramanya, and P. Shankar. 2009. Lentiviral delivery of short hairpin RNAs. Advanced drug delivery reviews 61:732-745.

192. Mansouri, M., K. Viswanathan, J. L. Douglas, J. Hines, J. Gustin, A. V. Moses, and K. Fruh. 2009. Molecular mechanism of BST2/tetherin downregulation by K5/MIR2 of Kaposi's sarcoma-associated herpesvirus. Journal of virology 83:9672-9681.

193. Marschall, M., S. Feichtinger, and J. Milbradt. 2011. Regulatory roles of protein kinases in cytomegalovirus replication. Advances in virus research 80:69-101.

194. Martinez-Guzman, D., T. Rickabaugh, T. T. Wu, H. Brown, S. Cole, M. J. Song, L. Tong, and R. Sun. 2003. Transcription program of murine gammaherpesvirus 68. Journal of virology 77:10488-10503.

195. Maurer, U. E., B. Sodeik, and K. Grunewald. 2008. Native 3D intermediates of membrane fusion in herpes simplex virus 1 entry. Proceedings of the National Academy of Sciences of the United States of America 105:1055910564.

196. May, J. S., B. D. de Lima, S. Colaco, and P. G. Stevenson. 2005. Intercellular gamma-herpesvirus dissemination involves co-ordinated intracellular membrane protein transport. Traffic 6:780-793.

197. May, J. S., J. Walker, S. Colaco, and P. G. Stevenson. 2005. The murine gammaherpesvirus 68 ORF27 gene product contributes to intercellular viral spread. Journal of virology 79:5059-5068.

198. McDonald, D., M. A. Vodicka, G. Lucero, T. M. Svitkina, G. G. Borisy, M. Emerman, and T. J. Hope. 2002. Visualization of the intracellular behavior of HIV in living cells. The Journal of cell biology 159:441-452.

199. McGeoch, D. J., F. J. Rixon, and A. J. Davison. 2006. Topics in herpesvirus genomics and evolution. Virus research 117:90-104.

200. McKnight, J. L., T. M. Kristie, and B. Roizman. 1987. Binding of the virion protein mediating alpha gene induction in herpes simplex virus 1-infected cells to its cis site requires cellular proteins. Proceedings of the National Academy of Sciences of the United States of America 84:7061-7065.

201. McLean, W. H., and E. B. Lane. 1995. Intermediate filaments in disease. Current opinion in cell biology 7:118-125.

202. McMillan, T. N., and D. C. Johnson. 2001. Cytoplasmic domain of herpes simplex virus gE causes accumulation in the trans-Golgi network, a site of virus envelopment and sorting of virions to cell junctions. Journal of virology 75:1928-1940. 
203. McNab, A. R., P. Desai, S. Person, L. L. Roof, D. R. Thomsen, W. W. Newcomb, J. C. Brown, and F. L. Homa. 1998. The product of the herpes simplex virus type 1 UL25 gene is required for encapsidation but not for cleavage of replicated viral DNA. Journal of virology 72:1060-1070.

204. Meckes, D. G., Jr., and J. W. Wills. 2007. Dynamic interactions of the UL16 tegument protein with the capsid of herpes simplex virus. Journal of virology 81:13028-13036.

205. Melancon, J. M., R. E. Luna, T. P. Foster, and K. G. Kousoulas. 2005. Herpes simplex virus type $1 \mathrm{gK}$ is required for gB-mediated virus-induced cell fusion, while neither $\mathrm{gB}$ and $\mathrm{gK}$ nor $\mathrm{gB}$ and UL20p function redundantly in virion deenvelopment. Journal of virology 79:299-313.

206. Mesri, E. A., E. Cesarman, L. Arvanitakis, S. Rafii, M. A. Moore, D. N. Posnett, D. M. Knowles, and A. S. Asch. 1996. Human herpesvirus8/Kaposi's sarcoma-associated herpesvirus is a new transmissible virus that infects B cells. The Journal of experimental medicine 183:2385-2390.

207. Messerle, M., I. Crnkovic, W. Hammerschmidt, H. Ziegler, and U. H. Koszinowski. 1997. Cloning and mutagenesis of a herpesvirus genome as an infectious bacterial artificial chromosome. Proceedings of the National Academy of Sciences of the United States of America 94:14759-14763.

208. Mettenleiter, T. C. 2004. Budding events in herpesvirus morphogenesis. Virus research 106:167-180.

209. Mettenleiter, T. C. 2002. Herpesvirus assembly and egress. Journal of virology 76:1537-1547.

210. Mettenleiter, T. C. 2006. Intriguing interplay between viral proteins during herpesvirus assembly or: the herpesvirus assembly puzzle. Veterinary microbiology 113:163-169.

211. Mettenleiter, T. C., B. G. Klupp, and H. Granzow. 2009. Herpesvirus assembly: an update. Virus research 143:222-234.

212. Mettenleiter, T. C., and T. Minson. 2006. Egress of alphaherpesviruses. Journal of virology 80:1610-1611; author reply 1611-1612.

213. Mettenleiter, T. C., F. Muller, H. Granzow, and B. G. Klupp. 2013. The way out: what we know and do not know about herpesvirus nuclear egress. Cellular microbiology 15:170-178.

214. Miranda-Saksena, M., R. A. Boadle, P. Armati, and A. L. Cunningham. 2002. In rat dorsal root ganglion neurons, herpes simplex virus type 1 tegument forms in the cytoplasm of the cell body. Journal of virology 76:9934-9951.

215. Moore, P. S., S. J. Gao, G. Dominguez, E. Cesarman, O. Lungu, D. M. Knowles, R. Garber, P. E. Pellett, D. J. McGeoch, and Y. Chang. 1996. Primary characterization of a herpesvirus agent associated with Kaposi's sarcomae. Journal of virology 70:549-558.

216. Moore, P. S., L. A. Kingsley, S. D. Holmberg, T. Spira, P. Gupta, D. R. Hoover, J. P. Parry, L. J. Conley, H. W. Jaffe, and Y. Chang. 1996. Kaposi's sarcoma-associated herpesvirus infection prior to onset of Kaposi's sarcoma. AIDS 10:175-180. 
217. Morrison, L. A., and G. S. DeLassus. 2011. Breach of the nuclear lamina during assembly of herpes simplex viruses. Nucleus 2:271-276.

218. Mothes, W., N. M. Sherer, J. Jin, and P. Zhong. 2010. Virus cell-to-cell transmission. Journal of virology 84:8360-8368.

219. Muranyi, W., J. Haas, M. Wagner, G. Krohne, and U. H. Koszinowski. 2002. Cytomegalovirus recruitment of cellular kinases to dissolve the nuclear lamina. Science 297:854-857.

220. Myoung, J., and D. Ganem. 2011. Active lytic infection of human primary tonsillar B cells by KSHV and its noncytolytic control by activated CD4+ T cells. The Journal of clinical investigation 121:1130-1140.

221. Myoung, J., and D. Ganem. 2011. Infection of primary human tonsillar lymphoid cells by KSHV reveals frequent but abortive infection of T cells. Virology 413:1-11.

222. Nadal, M., P. J. Mas, A. G. Blanco, C. Arnan, M. Sola, D. J. Hart, and M. Coll. 2010. Structure and inhibition of herpesvirus DNA packaging terminase nuclease domain. Proceedings of the National Academy of Sciences of the United States of America 107:16078-16083.

223. Nagaike, K., Y. Mori, Y. Gomi, H. Yoshii, M. Takahashi, M. Wagner, U. Koszinowski, and K. Yamanishi. 2004. Cloning of the varicella-zoster virus genome as an infectious bacterial artificial chromosome in Escherichia coli. Vaccine 22:4069-4074.

224. Nalwanga, D., S. Rempel, B. Roizman, and J. D. Baines. 1996. The UL 16 gene product of herpes simplex virus 1 is a virion protein that colocalizes with intranuclear capsid proteins. Virology 226:236-242.

225. Naranatt, P. P., H. H. Krishnan, M. S. Smith, and B. Chandran. 2005. Kaposi's sarcoma-associated herpesvirus modulates microtubule dynamics via RhoA-GTP-diaphanous 2 signaling and utilizes the dynein motors to deliver its DNA to the nucleus. Journal of virology 79:1191-1206.

226. Nealon, K., W. W. Newcomb, T. R. Pray, C. S. Craik, J. C. Brown, and D. H. Kedes. 2001. Lytic replication of Kaposi's sarcoma-associated herpesvirus results in the formation of multiple capsid species: isolation and molecular characterization of A, B, and C capsids from a gammaherpesvirus. Journal of virology 75:2866-2878.

227. Neil, S. J., T. Zang, and P. D. Bieniasz. 2008. Tetherin inhibits retrovirus release and is antagonized by HIV-1 Vpu. Nature 451:425-430.

228. Newcomb, W. W., F. P. Booy, and J. C. Brown. 2007. Uncoating the herpes simplex virus genome. Journal of molecular biology 370:633-642.

229. Newcomb, W. W., S. K. Cockrell, F. L. Homa, and J. C. Brown. 2009. Polarized DNA ejection from the herpesvirus capsid. Journal of molecular biology 392:885-894.

230. Newcomb, W. W., F. L. Homa, and J. C. Brown. 2006. Herpes simplex virus capsid structure: DNA packaging protein UL25 is located on the external surface of the capsid near the vertices. Journal of virology 80:6286-6294. 
231. Newcomb, W. W., F. L. Homa, and J. C. Brown. 2005. Involvement of the portal at an early step in herpes simplex virus capsid assembly. Journal of virology 79:10540-10546.

232. Newcomb, W. W., F. L. Homa, D. R. Thomsen, F. P. Booy, B. L. Trus, A. C. Steven, J. V. Spencer, and J. C. Brown. 1996. Assembly of the herpes simplex virus capsid: characterization of intermediates observed during cell-free capsid formation. Journal of molecular biology 263:432-446.

233. Newcomb, W. W., F. L. Homa, D. R. Thomsen, B. L. Trus, N. Cheng, A. Steven, F. Booy, and J. C. Brown. 1999. Assembly of the herpes simplex virus procapsid from purified components and identification of small complexes containing the major capsid and scaffolding proteins. Journal of virology 73:4239-4250.

234. Newcomb, W. W., F. L. Homa, D. R. Thomsen, Z. Ye, and J. C. Brown. 1994. Cell-free assembly of the herpes simplex virus capsid. Journal of virology 68:6059-6063.

235. Newcomb, W. W., R. M. Juhas, D. R. Thomsen, F. L. Homa, A. D. Burch, S. K. Weller, and J. C. Brown. 2001. The UL6 gene product forms the portal for entry of DNA into the herpes simplex virus capsid. Journal of virology 75:10923-10932.

236. Newcomb, W. W., B. L. Trus, N. Cheng, A. C. Steven, A. K. Sheaffer, D. J. Tenney, S. K. Weller, and J. C. Brown. 2000. Isolation of herpes simplex virus procapsids from cells infected with a protease-deficient mutant virus. Journal of virology 74:1663-1673.

237. Noh, C. W., H. J. Cho, H. R. Kang, H. Y. Jin, S. Lee, H. Deng, T. T. Wu, V. Arumugaswami, R. Sun, and M. J. Song. 2012. The virion-associated open reading frame 49 of murine gammaherpesvirus 68 promotes viral replication both in vitro and in vivo as a derepressor of RTA. Journal of virology 86:1109-1118.

238. O'Connor, C. M., B. Damania, and D. H. Kedes. 2003. De novo infection with rhesus monkey rhadinovirus leads to the accumulation of multiple intranuclear capsid species during lytic replication but favors the release of genome-containing virions. Journal of virology 77:13439-13447.

239. O'Connor, C. M., and D. H. Kedes. 2006. Mass spectrometric analyses of purified rhesus monkey rhadinovirus reveal 33 virion-associated proteins. Journal of virology 80:1574-1583.

240. Ogasawara, M., T. Suzutani, I. Yoshida, and M. Azuma. 2001. Role of the UL25 gene product in packaging DNA into the herpes simplex virus capsid: location of UL25 product in the capsid and demonstration that it binds DNA. Journal of virology 75:1427-1436.

241. Ojala, P. M., B. Sodeik, M. W. Ebersold, U. Kutay, and A. Helenius. 2000. Herpes simplex virus type 1 entry into host cells: reconstitution of capsid binding and uncoating at the nuclear pore complex in vitro. Molecular and cellular biology 20:4922-4931. 
242. Oshima, S., T. Daikoku, S. Shibata, H. Yamada, F. Goshima, and Y. Nishiyama. 1998. Characterization of the UL16 gene product of herpes simplex virus type 2. Archives of virology 143:863-880.

243. Pante, N., and M. Kann. 2002. Nuclear pore complex is able to transport macromolecules with diameters of about $39 \mathrm{~nm}$. Molecular biology of the cell 13:425-434.

244. Pardieu, C., R. Vigan, S. J. Wilson, A. Calvi, T. Zang, P. Bieniasz, P. Kellam, G. J. Towers, and S. J. Neil. 2010. The RING-CH ligase K5 antagonizes restriction of KSHV and HIV-1 particle release by mediating ubiquitindependent endosomal degradation of tetherin. PLoS pathogens 6:e1000843.

245. Pasdeloup, D., F. Beilstein, A. P. Roberts, M. McElwee, D. McNab, and F. J. Rixon. 2010. Inner tegument protein pUL37 of herpes simplex virus type 1 is involved in directing capsids to the trans-Golgi network for envelopment. The Journal of general virology 91:2145-2151.

246. Patel, A. H., F. J. Rixon, C. Cunningham, and A. J. Davison. 1996. Isolation and characterization of herpes simplex virus type 1 mutants defective in the UL6 gene. Virology 217:111-123.

247. Patterson, D., J. Bleskan, K. Gardiner, and J. Bowersox. 1999. Human phosphoribosylformylglycineamide amidotransferase (FGARAT): regional mapping, complete coding sequence, isolation of a functional genomic clone, and DNA sequence analysis. Gene 239:381-391.

248. Paulose-Murphy, M., N. K. Ha, C. Xiang, Y. Chen, L. Gillim, R. Yarchoan, P. Meltzer, M. Bittner, J. Trent, and S. Zeichner. 2001. Transcription program of human herpesvirus 8 (kaposi's sarcoma-associated herpesvirus). Journal of virology 75:4843-4853.

249. Person, S., S. Laquerre, P. Desai, and J. Hempel. 1993. Herpes simplex virus type 1 capsid protein, VP21, originates within the UL26 open reading frame. The Journal of general virology 74 ( Pt 10):2269-2273.

250. Plafker, S. M., and W. Gibson. 1998. Cytomegalovirus assembly protein precursor and proteinase precursor contain two nuclear localization signals that mediate their own nuclear translocation and that of the major capsid protein. Journal of virology 72:7722-7732.

251. Ploubidou, A., and M. Way. 2001. Viral transport and the cytoskeleton. Current opinion in cell biology 13:97-105.

252. Plow, E. F., T. A. Haas, L. Zhang, J. Loftus, and J. W. Smith. 2000. Ligand binding to integrins. The Journal of biological chemistry 275:21785-21788.

253. Poon, A. P., and B. Roizman. 1993. Characterization of a temperaturesensitive mutant of the UL15 open reading frame of herpes simplex virus 1 . Journal of virology 67:4497-4503.

254. Preston, V. G., J. Murray, C. M. Preston, I. M. McDougall, and N. D. Stow. 2008. The UL25 gene product of herpes simplex virus type 1 is involved in uncoating of the viral genome. Journal of virology 82:6654-6666.

255. Radtke, K., K. Dohner, and B. Sodeik. 2006. Viral interactions with the cytoskeleton: a hitchhiker's guide to the cell. Cellular microbiology 8:387400. 
256. Radtke, K., D. Kieneke, A. Wolfstein, K. Michael, W. Steffen, T. Scholz, A. Karger, and B. Sodeik. 2010. Plus- and minus-end directed microtubule motors bind simultaneously to herpes simplex virus capsids using different inner tegument structures. PLoS pathogens 6:e1000991.

257. Rappocciolo, G., H. R. Hensler, M. Jais, T. A. Reinhart, A. Pegu, F. J. Jenkins, and C. R. Rinaldo. 2008. Human herpesvirus 8 infects and replicates in primary cultures of activated B lymphocytes through DC-SIGN. Journal of virology 82:4793-4806.

258. Rappocciolo, G., F. J. Jenkins, H. R. Hensler, P. Piazza, M. Jais, L. Borowski, S. C. Watkins, and C. R. Rinaldo, Jr. 2006. DC-SIGN is a receptor for human herpesvirus 8 on dendritic cells and macrophages. J Immunol 176:1741-1749.

259. Renne, R., W. Zhong, B. Herndier, M. McGrath, N. Abbey, D. Kedes, and D. Ganem. 1996. Lytic growth of Kaposi's sarcoma-associated herpesvirus (human herpesvirus 8) in culture. Nature medicine 2:342-346.

260. Reynolds, A. E., E. G. Wills, R. J. Roller, B. J. Ryckman, and J. D. Baines. 2002. Ultrastructural localization of the herpes simplex virus type 1 UL31, UL34, and US3 proteins suggests specific roles in primary envelopment and egress of nucleocapsids. Journal of virology 76:8939-8952.

261. Rietdorf, J., A. Ploubidou, I. Reckmann, A. Holmstrom, F. Frischknecht, M. Zettl, T. Zimmermann, and M. Way. 2001. Kinesin-dependent movement on microtubules precedes actin-based motility of vaccinia virus. Nature cell biology 3:992-1000.

262. Rivera-Molina, Y. A., F. P. Martinez, and Q. Tang. 2013. Nuclear domain 10 of the viral aspect. World journal of virology 2:110-122.

263. Rixon, F. J. 1993. Structure and assembly of herpesviruses. Seminars in Virology:135-144.

264. Rixon, F. J., C. Addison, A. McGregor, S. J. Macnab, P. Nicholson, V. G. Preston, and J. D. Tatman. 1996. Multiple interactions control the intracellular localization of the herpes simplex virus type 1 capsid proteins. The Journal of general virology 77 ( Pt 9):2251-2260.

265. Rixon, M. W., B. B. Gourlie, D. A. Kaplan, J. Schlom, and P. S. Mezes. 1993. Preferential use of a $\mathrm{H}$ chain $\mathrm{V}$ region in antitumor-associated glycoprotein72 monoclonal antibodies. J Immunol 151:6559-6568.

266. Rowinsky, E. K., N. Onetto, R. M. Canetta, and S. G. Arbuck. 1992. Taxol: the first of the taxanes, an important new class of antitumor agents. Seminars in oncology 19:646-662.

267. Rozen, R., N. Sathish, Y. Li, and Y. Yuan. 2008. Virion-wide protein interactions of Kaposi's sarcoma-associated herpesvirus. Journal of virology 82:4742-4750.

268. Rozenblatt-Rosen, O., R. C. Deo, M. Padi, G. Adelmant, M. A. Calderwood, T. Rolland, M. Grace, A. Dricot, M. Askenazi, M. Tavares, S. J. Pevzner, F. Abderazzaq, D. Byrdsong, A. R. Carvunis, A. A. Chen, J. Cheng, M. Correll, M. Duarte, C. Fan, M. C. Feltkamp, S. B. Ficarro, R. Franchi, B. K. Garg, N. Gulbahce, T. Hao, A. M. Holthaus, R. James, A. Korkhin, L. Litovchick, J. C. 
Mar, T. R. Pak, S. Rabello, R. Rubio, Y. Shen, S. Singh, J. M. Spangle, M.

Tasan, S. Wanamaker, J. T. Webber, J. Roecklein-Canfield, E. Johannsen, A. L. Barabasi, R. Beroukhim, E. Kieff, M. E. Cusick, D. E. Hill, K. Munger, J. A. Marto, J. Quackenbush, F. P. Roth, J. A. DeCaprio, and M. Vidal. 2012. Interpreting cancer genomes using systematic host network perturbations by tumour virus proteins. Nature 487:491-495.

269. Russo, J. J., R. A. Bohenzky, M. C. Chien, J. Chen, M. Yan, D. Maddalena, J. P. Parry, D. Peruzzi, I. S. Edelman, Y. Chang, and P. S. Moore. 1996. Nucleotide sequence of the Kaposi sarcoma-associated herpesvirus (HHV8). Proceedings of the National Academy of Sciences of the United States of America 93:14862-14867.

270. Ryan, K. J., C. G. Ray, J. C. Sherris, and Ralph Erskine Conrad Memorial Fund. 2004. Sherris medical microbiology : an introduction to infectious diseases, 4th ed. McGraw-Hill, New York.

271. Sakuma, T., T. Noda, S. Urata, Y. Kawaoka, and J. Yasuda. 2009. Inhibition of Lassa and Marburg virus production by tetherin. Journal of virology 83:2382-2385.

272. Sam, M. D., B. T. Evans, D. M. Coen, and J. M. Hogle. 2009. Biochemical, biophysical, and mutational analyses of subunit interactions of the human cytomegalovirus nuclear egress complex. Journal of virology 83:2996-3006.

273. Sanchez, V., E. Sztul, and W. J. Britt. 2000. Human cytomegalovirus pp28 (UL99) localizes to a cytoplasmic compartment which overlaps the endoplasmic reticulum-golgi-intermediate compartment. Journal of virology 74:3842-3851.

274. Sander, G., A. Konrad, M. Thurau, E. Wies, R. Leubert, E. Kremmer, H. Dinkel, T. Schulz, F. Neipel, and M. Sturzl. 2008. Intracellular localization map of human herpesvirus 8 proteins. Journal of virology 82:1908-1922.

275. Sathish, N., X. Wang, and Y. Yuan. 2012. Tegument Proteins of Kaposi's Sarcoma-Associated Herpesvirus and Related Gamma-Herpesviruses. Frontiers in microbiology 3:98.

276. Sathish, N., F. X. Zhu, E. E. Golub, Q. Liang, and Y. Yuan. 2011. Mechanisms of autoinhibition of IRF-7 and a probable model for inactivation of IRF-7 by Kaposi's sarcoma-associated herpesvirus protein ORF45. The Journal of biological chemistry 286:746-756.

277. Sathish, N., F. X. Zhu, and Y. Yuan. 2009. Kaposi's sarcoma-associated herpesvirus ORF45 interacts with kinesin-2 transporting viral capsidtegument complexes along microtubules. PLoS pathogens 5:e1000332.

278. Schimmer, C., and A. Neubauer. 2003. The equine herpesvirus 1 UL11 gene product localizes to the trans-golgi network and is involved in cell-to-cell spread. Virology 308:23-36.

279. Schlieker, C., G. A. Korbel, L. M. Kattenhorn, and H. L. Ploegh. 2005. A deubiquitinating activity is conserved in the large tegument protein of the herpesviridae. Journal of virology 79:15582-15585. 
280. Schulz, T. F. 2000. Kaposi's sarcoma-associated herpesvirus (human herpesvirus 8): epidemiology and pathogenesis. The Journal of antimicrobial chemotherapy 45 Suppl T3:15-27.

281. Searles, R. P., E. P. Bergquam, M. K. Axthelm, and S. W. Wong. 1999. Sequence and genomic analysis of a Rhesus macaque rhadinovirus with similarity to Kaposi's sarcoma-associated herpesvirus/human herpesvirus 8. Journal of virology 73:3040-3053.

282. Seisenberger, G., M. U. Ried, T. Endress, H. Buning, M. Hallek, and C. Brauchle. 2001. Real-time single-molecule imaging of the infection pathway of an adeno-associated virus. Science 294:1929-1932.

283. Seo, J. Y., and W. J. Britt. 2006. Sequence requirements for localization of human cytomegalovirus tegument protein pp28 to the virus assembly compartment and for assembly of infectious virus. Journal of virology 80:5611-5626.

284. Sewatanon, J., and P. D. Ling. 2013. Murine gammaherpesvirus 68 ORF75c contains ubiquitin E3 ligase activity and requires PML SUMOylation but not other known cellular PML regulators, CK2 and E6AP, to mediate PML degradation. Virology 440:140-149.

285. Sheaffer, A. K., W. W. Newcomb, M. Gao, D. Yu, S. K. Weller, J. C. Brown, and D. J. Tenney. 2001. Herpes simplex virus DNA cleavage and packaging proteins associate with the procapsid prior to its maturation. Journal of virology 75:687-698.

286. Shen, S., H. Guo, and H. Deng. 2014. Murine gammaherpesvirus-68 ORF38 encodes a tegument protein and is packaged into virions during secondary envelopment. Protein \& cell.

287. Shepherd, F. A., E. Maher, C. Cardella, E. Cole, P. Greig, J. A. Wade, and G. Levy. 1997. Treatment of Kaposi's sarcoma after solid organ transplantation. Journal of clinical oncology : official journal of the American Society of Clinical Oncology 15:2371-2377.

288. Sherer, N. M., J. Jin, and W. Mothes. 2010. Directional spread of surfaceassociated retroviruses regulated by differential virus-cell interactions. Journal of virology 84:3248-3258.

289. Shu, M., B. Taddeo, W. Zhang, and B. Roizman. 2013. Selective degradation of mRNAs by the HSV host shutoff RNase is regulated by the UL47 tegument protein. Proceedings of the National Academy of Sciences of the United States of America 110:E1669-1675.

290. Silva, M. C., J. Schroer, and T. Shenk. 2005. Human cytomegalovirus cell-tocell spread in the absence of an essential assembly protein. Proceedings of the National Academy of Sciences of the United States of America 102:20812086.

291. Sinclair, J., and P. Sissons. 2006. Latency and reactivation of human cytomegalovirus. The Journal of general virology 87:1763-1779.

292. Sinzger, C., M. Digel, and G. Jahn. 2008. Cytomegalovirus cell tropism. Current topics in microbiology and immunology 325:63-83. 
293. Skepper, J. N., A. Whiteley, H. Browne, and A. Minson. 2001. Herpes simplex virus nucleocapsids mature to progeny virions by an envelopment -$>$ deenvelopment --> reenvelopment pathway. Journal of virology 75:56975702.

294. Smiley, J. R. 2004. Herpes simplex virus virion host shutoff protein: immune evasion mediated by a viral RNase? Journal of virology 78:1063-1068.

295. Sodeik, B., M. W. Ebersold, and A. Helenius. 1997. Microtubule-mediated transport of incoming herpes simplex virus 1 capsids to the nucleus. The Journal of cell biology 136:1007-1021.

296. Song, M. J., S. Hwang, W. H. Wong, T. T. Wu, S. Lee, H. I. Liao, and R. Sun. 2005. Identification of viral genes essential for replication of murine gammaherpesvirus 68 using signature-tagged mutagenesis. Proceedings of the National Academy of Sciences of the United States of America 102:38053810.

297. Soulier, J., L. Grollet, E. Oksenhendler, P. Cacoub, D. Cazals-Hatem, P. Babinet, M. F. d'Agay, J. P. Clauvel, M. Raphael, L. Degos, and et al. 1995. Kaposi's sarcoma-associated herpesvirus-like DNA sequences in multicentric Castleman's disease. Blood 86:1276-1280.

298. Spear, P. G., and B. Roizman. 1972. Proteins specified by herpes simplex virus. V. Purification and structural proteins of the herpesvirion. Journal of virology 9:143-159.

299. Steven, A. C., B. L. Trus, F. P. Booy, N. Cheng, A. Zlotnick, J. R. Caston, and J. F. Conway. 1997. The making and breaking of symmetry in virus capsid assembly: glimpses of capsid biology from cryoelectron microscopy. FASEB journal : official publication of the Federation of American Societies for Experimental Biology 11:733-742.

300. Suomalainen, M., M. Y. Nakano, S. Keller, K. Boucke, R. P. Stidwill, and U. F. Greber. 1999. Microtubule-dependent plus- and minus end-directed motilities are competing processes for nuclear targeting of adenovirus. The Journal of cell biology 144:657-672.

301. Taddeo, B., and B. Roizman. 2006. The virion host shutoff protein (UL41) of herpes simplex virus 1 is an endoribonuclease with a substrate specificity similar to that of RNase A. Journal of virology 80:9341-9345.

302. Taddeo, B., W. Zhang, and B. Roizman. 2006. The U(L)41 protein of herpes simplex virus 1 degrades RNA by endonucleolytic cleavage in absence of other cellular or viral proteins. Proceedings of the National Academy of Sciences of the United States of America 103:2827-2832.

303. Takakuwa, H., F. Goshima, T. Koshizuka, T. Murata, T. Daikoku, and Y. Nishiyama. 2001. Herpes simplex virus encodes a virion-associated protein which promotes long cellular processes in over-expressing cells. Genes to cells : devoted to molecular \& cellular mechanisms 6:955-966.

304. Tanaka, M., H. Kagawa, Y. Yamanashi, T. Sata, and Y. Kawaguchi. 2003. Construction of an excisable bacterial artificial chromosome containing a fulllength infectious clone of herpes simplex virus type 1: viruses reconstituted 
from the clone exhibit wild-type properties in vitro and in vivo. Journal of virology 77:1382-1391.

305. Taus, N. S., B. Salmon, and J. D. Baines. 1998. The herpes simplex virus 1 UL 17 gene is required for localization of capsids and major and minor capsid proteins to intranuclear sites where viral DNA is cleaved and packaged. Virology 252:115-125.

306. Tavalai, N., and T. Stamminger. 2008. New insights into the role of the subnuclear structure ND10 for viral infection. Biochimica et biophysica acta 1783:2207-2221.

307. Taylor, J. F., A. C. Templeton, C. L. Vogel, J. L. Ziegler, and S. K. Kyalwazi. 1971. Kaposi's sarcoma in Uganda: a clinico-pathological study. International journal of cancer. Journal international du cancer 8:122-135.

308. Taylor, M. P., O. O. Koyuncu, and L. W. Enquist. 2011. Subversion of the actin cytoskeleton during viral infection. Nature reviews. Microbiology 9:427-439.

309. Tengelsen, L. A., N. E. Pederson, P. R. Shaver, M. W. Wathen, and F. L. Homa. 1993. Herpes simplex virus type 1 DNA cleavage and encapsidation require the product of the UL28 gene: isolation and characterization of two UL28 deletion mutants. Journal of virology 67:3470-3480.

310. Thurlow, J. K., M. Murphy, N. D. Stow, and V. G. Preston. 2006. Herpes simplex virus type 1 DNA-packaging protein UL17 is required for efficient binding of UL25 to capsids. Journal of virology 80:2118-2126.

311. Tirabassi, R. S., and L. W. Enquist. 1999. Mutation of the YXXL endocytosis motif in the cytoplasmic tail of pseudorabies virus gE. Journal of virology 73:2717-2728.

312. Tiscornia, G., O. Singer, and I. M. Verma. 2006. Design and cloning of lentiviral vectors expressing small interfering RNAs. Nature protocols 1:234240.

313. Toropova, K., J. B. Huffman, F. L. Homa, and J. F. Conway. 2011. The herpes simplex virus 1 UL17 protein is the second constituent of the capsid vertex-specific component required for DNA packaging and retention. Journal of virology 85:7513-7522.

314. Trinczek, B., J. Biernat, K. Baumann, E. M. Mandelkow, and E. Mandelkow. 1995. Domains of tau protein, differential phosphorylation, and dynamic instability of microtubules. Molecular biology of the cell 6:18871902.

315. Trus, B. L., F. P. Booy, W. W. Newcomb, J. C. Brown, F. L. Homa, D. R. Thomsen, and A. C. Steven. 1996. The herpes simplex virus procapsid: structure, conformational changes upon maturation, and roles of the triplex proteins VP19c and VP23 in assembly. Journal of molecular biology 263:447462.

316. Trus, B. L., W. W. Newcomb, N. Cheng, G. Cardone, L. Marekov, F. L. Homa, J. C. Brown, and A. C. Steven. 2007. Allosteric signaling and a nuclear exit strategy: binding of UL25/UL17 heterodimers to DNA-Filled HSV-1 capsids. Molecular cell 26:479-489. 
317. Tsai, K., N. Thikmyanova, J. A. Wojcechowskyj, H. J. Delecluse, and P. M. Lieberman. 2011. EBV tegument protein BNRF1 disrupts DAXX-ATRX to activate viral early gene transcription. PLoS pathogens 7:e1002376.

318. Turcotte, S., J. Letellier, and R. Lippe. 2005. Herpes simplex virus type 1 capsids transit by the trans-Golgi network, where viral glycoproteins accumulate independently of capsid egress. Journal of virology 79:88478860.

319. Uldrick, T. S., and D. Whitby. 2011. Update on KSHV epidemiology, Kaposi Sarcoma pathogenesis, and treatment of Kaposi Sarcoma. Cancer letters 305:150-162.

320. Vale, R. D. 2000. AAA proteins. Lords of the ring. The Journal of cell biology 150:F13-19.

321. Vale, R. D. 2003. The molecular motor toolbox for intracellular transport. Cell 112:467-480.

322. Valpuesta, J. M., and J. L. Carrascosa. 1994. Structure of viral connectors and their function in bacteriophage assembly and DNA packaging. Quarterly reviews of biophysics 27:107-155.

323. Van Damme, N., D. Goff, C. Katsura, R. L. Jorgenson, R. Mitchell, M. C. Johnson, E. B. Stephens, and J. Guatelli. 2008. The interferon-induced protein BST-2 restricts HIV-1 release and is downregulated from the cell surface by the viral Vpu protein. Cell host \& microbe 3:245-252.

324. van Ooij, C. 2010. Virology: cleaving the tether. Nature reviews. Microbiology 8:385.

325. Varnum, S. M., D. N. Streblow, M. E. Monroe, P. Smith, K. J. Auberry, L. Pasa-Tolic, D. Wang, D. G. Camp, 2nd, K. Rodland, S. Wiley, W. Britt, T. Shenk, R. D. Smith, and J. A. Nelson. 2004. Identification of proteins in human cytomegalovirus (HCMV) particles: the HCMV proteome. Journal of virology 78:10960-10966.

326. Verhey, K. J., and J. W. Hammond. 2009. Traffic control: regulation of kinesin motors. Nature reviews. Molecular cell biology 10:765-777.

327. Virgin, H. W. t., P. Latreille, P. Wamsley, K. Hallsworth, K. E. Weck, A. J. Dal Canto, and S. H. Speck. 1997. Complete sequence and genomic analysis of murine gammaherpesvirus 68. Journal of virology 71:5894-5904.

328. Wagner, M., S. Jonjic, U. H. Koszinowski, and M. Messerle. 1999. Systematic excision of vector sequences from the BAC-cloned herpesvirus genome during virus reconstitution. Journal of virology 73:7056-7060.

329. Wang, H. Y., W. Lin, J. A. Dyck, J. M. Yeakley, Z. Songyang, L. C. Cantley, and X. D. Fu. 1998. SRPK2: a differentially expressed SR protein-specific kinase involved in mediating the interaction and localization of pre-mRNA splicing factors in mammalian cells. The Journal of cell biology 140:737-750.

330. Wang, L., H. Guo, N. Reyes, S. Lee, E. Bortz, F. Guo, R. Sun, L. Tong, and H. Deng. 2012. Distinct domains in ORF52 tegument protein mediate essential functions in murine gammaherpesvirus 68 virion tegumentation and secondary envelopment. Journal of virology 86:1348-1357. 
331. Ward, B. M. 2011. The taking of the cytoskeleton one two three: how viruses utilize the cytoskeleton during egress. Virology 411:244-250.

332. Wen, K. W., and B. Damania. 2010. Kaposi sarcoma-associated herpesvirus (KSHV): molecular biology and oncogenesis. Cancer letters 289:140-150.

333. White, C. A., N. D. Stow, A. H. Patel, M. Hughes, and V. G. Preston. 2003. Herpes simplex virus type 1 portal protein UL6 interacts with the putative terminase subunits UL15 and UL28. Journal of virology 77:6351-6358.

334. Wild, P., M. Engels, C. Senn, K. Tobler, U. Ziegler, E. M. Schraner, E. Loepfe, M. Ackermann, M. Mueller, and P. Walther. 2005. Impairment of nuclear pores in bovine herpesvirus 1-infected MDBK cells. Journal of virology 79:1071-1083.

335. Willard, M. 2002. Rapid directional translocations in virus replication. Journal of virology 76:5220-5232.

336. Wolfstein, A., C. H. Nagel, K. Radtke, K. Dohner, V. J. Allan, and B. Sodeik. 2006. The inner tegument promotes herpes simplex virus capsid motility along microtubules in vitro. Traffic 7:227-237.

337. Wong, S. W., E. P. Bergquam, R. M. Swanson, F. W. Lee, S. M. Shiigi, N. A. Avery, J. W. Fanton, and M. K. Axthelm. 1999. Induction of B cell hyperplasia in simian immunodeficiency virus-infected rhesus macaques with the simian homologue of Kaposi's sarcoma-associated herpesvirus. The Journal of experimental medicine 190:827-840.

338. Wood, C., and W. Harrington, Jr. 2005. AIDS and associated malignancies. Cell research 15:947-952.

339. Wood, L. J., M. K. Baxter, S. M. Plafker, and W. Gibson. 1997. Human cytomegalovirus capsid assembly protein precursor (pUL80.5) interacts with itself and with the major capsid protein (pUL86) through two different domains. Journal of virology 71:179-190.

340. Woodson, E. N., M. S. Anderson, M. S. Loftus, and D. H. Kedes. 2014. Progressive Accumulation of Activated ERK2 within Highly Stable ORF45Containing Nuclear Complexes Promotes Lytic Gammaherpesvirus Infection. PLoS pathogens 10:e1004066.

341. Woodson, E. N., and D. H. Kedes. 2012. Distinct roles for extracellular signal-regulated kinase 1 (ERK1) and ERK2 in the structure and production of a primate gammaherpesvirus. Journal of virology 86:9721-9736.

342. Wozniak, M. J., R. Milner, and V. Allan. 2004. N-terminal kinesins: many and various. Traffic 5:400-410.

343. Wu, C. A., N. J. Nelson, D. J. McGeoch, and M. D. Challberg. 1988. Identification of herpes simplex virus type 1 genes required for origindependent DNA synthesis. Journal of virology 62:435-443.

344. Yamazaki, H., T. Nakata, Y. Okada, and N. Hirokawa. 1995. KIF3A/B: a heterodimeric kinesin superfamily protein that works as a microtubule plus end-directed motor for membrane organelle transport. The Journal of cell biology 130:1387-1399. 
345. Ye, F., X. Lei, and S. J. Gao. 2011. Mechanisms of Kaposi's SarcomaAssociated Herpesvirus Latency and Reactivation. Advances in virology 2011.

346. Yeh, P. C., D. G. Meckes, Jr., and J. W. Wills. 2008. Analysis of the interaction between the UL11 and UL16 tegument proteins of herpes simplex virus. Journal of virology 82:10693-10700.

347. Yu, D., G. A. Smith, L. W. Enquist, and T. Shenk. 2002. Construction of a self-excisable bacterial artificial chromosome containing the human cytomegalovirus genome and mutagenesis of the diploid TRL/IRL13 gene. Journal of virology 76:2316-2328.

348. Yu, D., and S. K. Weller. 1998. Genetic analysis of the UL 15 gene locus for the putative terminase of herpes simplex virus type 1. Virology 243:32-44.

349. Yu, X. K., C. M. O'Connor, I. Atanasov, B. Damania, D. H. Kedes, and Z. H. Zhou. 2003. Three-dimensional structures of the A, B, and C capsids of rhesus monkey rhadinovirus: insights into gammaherpesvirus capsid assembly, maturation, and DNA packaging. Journal of virology 77:1318213193.

350. Zaichick, S. V., K. P. Bohannon, A. Hughes, P. J. Sollars, G. E. Pickard, and G. A. Smith. 2013. The herpesvirus VP1/2 protein is an effector of dyneinmediated capsid transport and neuroinvasion. Cell host \& microbe 13:193203.

351. Zenner, H. L., R. Mauricio, G. Banting, and C. M. Crump. 2013. Herpes simplex virus 1 counteracts tetherin restriction via its virion host shutoff activity. Journal of virology 87:13115-13123.

352. Zhang, W., and S. J. Gao. 2012. Exploitation of Cellular Cytoskeletons and Signaling Pathways for Cell Entry by Kaposi's Sarcoma-Associated Herpesvirus and the Closely Related Rhesus Rhadinovirus. Pathogens 1:102127.

353. Zhang, W., F. Zhou, W. Greene, and S. J. Gao. 2010. Rhesus rhadinovirus infection of rhesus fibroblasts occurs through clathrin-mediated endocytosis. Journal of virology 84:11709-11717.

354. Zhong, P., L. M. Agosto, J. B. Munro, and W. Mothes. 2013. Cell-to-cell transmission of viruses. Current opinion in virology 3:44-50.

355. Zhou, F. C., Y. J. Zhang, J. H. Deng, X. P. Wang, H. Y. Pan, E. Hettler, and S. J. Gao. 2002. Efficient infection by a recombinant Kaposi's sarcoma-associated herpesvirus cloned in a bacterial artificial chromosome: application for genetic analysis. Journal of virology 76:6185-6196.

356. Zhou, Z. H., D. H. Chen, J. Jakana, F. J. Rixon, and W. Chiu. 1999. Visualization of tegument-capsid interactions and DNA in intact herpes simplex virus type 1 virions. Journal of virology 73:3210-3218.

357. Zhou, Z. H., M. Dougherty, J. Jakana, J. He, F. J. Rixon, and W. Chiu. 2000. Seeing the herpesvirus capsid at 8.5 A. Science 288:877-880.

358. Zhu, F. X., J. M. Chong, L. Wu, and Y. Yuan. 2005. Virion proteins of Kaposi's sarcoma-associated herpesvirus. Journal of virology 79:800-811. 
359. Zhu, F. X., T. Cusano, and Y. Yuan. 1999. Identification of the immediate-early transcripts of Kaposi's sarcoma-associated herpesvirus. Journal of virology 73:5556-5567.

360. Zhu, F. X., S. M. King, E. J. Smith, D. E. Levy, and Y. Yuan. 2002. A Kaposi's sarcoma-associated herpesviral protein inhibits virus-mediated induction of type I interferon by blocking IRF-7 phosphorylation and nuclear accumulation. Proceedings of the National Academy of Sciences of the United States of America 99:5573-5578.

361. Zhu, F. X., X. Li, F. Zhou, S. J. Gao, and Y. Yuan. 2006. Functional characterization of Kaposi's sarcoma-associated herpesvirus ORF45 by bacterial artificial chromosome-based mutagenesis. Journal of virology 80:12187-12196.

362. Zhu, F. X., N. Sathish, and Y. Yuan. 2010. Antagonism of host antiviral responses by Kaposi's sarcoma-associated herpesvirus tegument protein ORF45. PloS one 5:e10573.

363. Zhu, F. X., and Y. Yuan. 2003. The ORF45 protein of Kaposi's sarcomaassociated herpesvirus is associated with purified virions. Journal of virology 77:4221-4230.

364. Zhu, Z., M. D. Gershon, Y. Hao, R. T. Ambron, C. A. Gabel, and A. A. Gershon. 1995. Envelopment of varicella-zoster virus: targeting of viral glycoproteins to the trans-Golgi network. Journal of virology 69:7951-7959.

365. Zhu, Z., Y. Hao, M. D. Gershon, R. T. Ambron, and A. A. Gershon. 1996. Targeting of glycoprotein I (gE) of varicella-zoster virus to the trans-Golgi network by an AYRV sequence and an acidic amino acid-rich patch in the cytosolic domain of the molecule. Journal of virology 70:6563-6575. 